\title{
Alessandra Cazzola
}

\section{I paesaggi nelle campagne di Roma}


TESI

SCIENZE TECNOLOGICHE

$-7-$ 
1. Gabriele Paolinelli, La frammentazione del paesaggio periurbano. Criteri progettuali per la riqualificazione della piana di Firenze, 2003

2. Enrica Dall'Ara, Costruire per temi i paesaggi? Esiti spaziali della semantica nei parchi tematici europei, 2004

3. Maristella Storti, Il paesaggio storico nelle Cinque Terre: Individuazione di regole per azioni di progetto condivise, 2004

4. Massimo Carta, Progetti di territorio. La costruzione di nuove tecniche di rappresentazione nei Sistemi Informativi Territoriali, 2005

5. Emanuela Morelli, Disegnare linee nel paesaggio. Metodologie di progettazione paesistica delle grandi infrastrutture viarie, 2005

6. Fabio Lucchesi, Il territorio, il codice, la rappresentazione. Il disegno dello statuto dei luoghi, 2005 


\author{
Alessandra Cazzola
}

\title{
I paesaggi nelle campagne di Roma
}

Firenze University Press 2005 
I paesaggi nelle campagne di Roma / Alessandra Cazzola. - Firenze : Firenze university press, 2005.

(Tesi. Scienze Tecnologiche, 7)

Edizione elettronica disponibile su http://e-prints.unifi.it

Stampa a richiesta disponibile su http://epress.unifi.it

ISBN978-88-6453-119-9 (online)

ISBN 88-8453-399-6 (print)

711 (ed. 20)

Architettura del paesaggio

(C) 2005 Firenze University Press

Università degli Studi di Firenze

Firenze University Press

Borgo Albizi, 28

50122 Firenze, Italy

http://epress.unifi.it/

Printed in Italy 
UNIVERSITÀ DEGLI STUDI DI FIRENZE - FACOLTÀ DI ARCHITETTURA

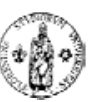

DOTTORATO DI RICERCA IN PROGETTAZIONE PAESISTICA

Dipartimento di Urbanistica e Pianificazione del Territorio

Coordinatore Prof. Giulio G. Rizzo

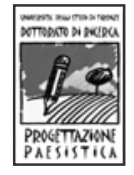

I paesaggi nelle Campagne di Roma

Ricerca di Alessandra Cazzola

Tutor

Prof. Andrea Tosi (Politecnico di Milano)

Co-Tutor

Prof. Carlo Alberto Garzonio (Università di Firenze)

Co-Tutor esterno

Prof. Massimo Olivieri (Università di Roma) 

A Nino

per $i$ sogni che non ha potuto realizzare 
Questo lavoro è tratto dalla tesi di dottorato di ricerca in «Progettazione Paesistica» discussa nel settembre 2003 presso la Facoltà di Architettura dell'Università degli Studi di Firenze.

Esso è frutto di uno sforzo individuale che ha tratto preziosi benefici dal supporto delle molte persone con cui mi sono confrontata all'interno e all'esterno dell'ambiente accademico.

Ringrazio Giulio G. Rizzo, coordinatore del Dottorato, per i continui stimoli durante l'intero periodo di ricerca; Andrea Tosi, tutor della ricerca, per gli spunti sugli aspetti metodologici che interessano il paesaggio agrario; Carlo Alberto Garzonio e Massimo Olivieri, co-tutor della ricerca, per gli aspetti più tecnici e per gli importanti suggerimenti riguardanti le specificità del territorio romano; tutti gli altri componenti il Collegio dei Docenti e i miei colleghi dottorandi, per i molti contributi di discussione, risultati determinanti nella stesura dello studio.

Tutta la mia gratitudine va inoltre a Gianni Beltrame, Lando Bortolotti, Vittoria Calzolari, Camillo Nucci, che, in occasioni di incontri diversi, hanno sostenuto e incoraggiato varie fasi della ricerca.

Un ringraziamento speciale va ad Antonella Galassi, Antonella Parisi Presicce e Graziella Priulla, per i costanti e sostanziali contributi alla revisione critica del lavoro e per la forza e gli sproni nei miei momenti di maggiore criticità. 


\section{INDICE}

Presentazione di Antonella Galassi

\section{Premessa}

Lo scenario di partenza

\section{PARTE PRIMA}

\section{UNA POSSIBILE METODOLOGIA PER LA LETTURA DEL PAESAGGIO AGRARIO}

Cap.1 La struttura del paesaggio agrario: gli elementi componenti e le loro relazioni

Le sistemazioni agrarie (il frazionamento fondiario, l'andamento morfologico dei terreni, la tessitura delle coltivazioni, le colture prevalenti)

L'insediamento rurale (la viabilità con il sistema degli accessi e dei percorsi poderali, la posizione del casale e degli annessi agricoli, i modi di costruire i confini)

La tipologia delle aziende e della loro conduzione

Cap.2 I principali cambiamenti del paesaggio agrario: alcune tra le cause principali della perdita di figurabilità

L'innovazione tecnologica

La riforma agraria

II processo di "urbanizzazione" e di "rururbanizzazione"

La Politica Agricola Comune dell'Unione Europea

Cap.3 Le ragioni di un'inversione di tendenza: il paesaggio agrario da ambito residuale ad elemento di connessione delle emergenze ambientali e paesistiche e "presidio" per il territorio

L'equilibrio produttivo ed economico

La protezione dell'ambiente e il riequilibrio ecologico

II riconoscimento dei "segni storici" per ritrovare l'identità dei luoghi

Cap.4 Lo stato di salute del paesaggio agrario: gli indicatori

Alcune considerazioni generali

Indicatori ed agricoltura (valori paesistici, valori ecologico-ambientali, valori produttivi)

Alcuni esempi: il Manuale AAA e la proposta della Commissione Agricoltura dell'Unione Europea 
PARTE SECONDA

IL CAMPO APPLICATIVO: IL PAESAGgIO AGRARIO DELLA CAMPAGNA ROMANA

Cap.5 Delimitazione e descrizione del campo applicativo

Quale è stata la delimitazione della campagna romana in diverse esperienze di ricerca e quali sono i suoi elementi strutturanti: da "campagna romana" ad "area romana"

Cap.6 Cause ed effetti delle principali trasformazioni nel paesaggio della campagna romana

La proprietà e la rendita: evoluzione del regime fondiario

Le opere di bonifica

L'infrastrutturazione del territorio

L'urbanizzazione e la nascita della città metropolitana

Cap.7 Le "campagne" dell'area romana oggi

La campagna delle grandi tenute

La campagna delle bonifiche

La campagna della città metropolitana

Abachi descrittivo-progettuali

Bibliografia specifica di riferimento

CONCLUSIONI

Esiti e possibili indirizzi di sviluppo

Come sono cambiati i rapporti tra gli elementi componenti: possibili criteri di progettazione paesistica compatibili con le attuali "campagne romane" 


\section{Presentazione}

La ricerca di dottorato di Alessandra Cazzola ha scelto di lavorare sul paesaggio, con un approccio di tipo teorico nella prima parte, per impostare il tema, e di tipo operativo nella seconda, centrando l'interesse sulla campagna romana.

A partire dal '600-'700 non c'è stato luogo più descritto e rappresentato della campagna romana con carte, disegni, dipinti, diari che mettevano in evidenza luci ed ombre di questo territorio. Un territorio, quello della campagna romana, che evocava (ed evoca) un paesaggio dove l'intreccio fra aree coltivate, valori naturali, insediamenti e monumenti storici produce varietà di forme ed identità tuttora riconoscibili, nonostante le molte trasformazioni subite nei secoli.

Su un attento lavoro di interpretazione dei luoghi e delle loro evoluzioni/alterazioni si fonda la tesi di dottorato presentata che tenta di portare un contributo nuovo alla discussione, ragionando sul ruolo del paesaggio agrario nell'area romana.

Leggendo la tesi, avevo già avuto l'impressione che questo lavoro sulla campagna romana o (come giustamente puntualizza Alessandra) sulle campagne romane ponesse l'attenzione su un tema tutt'altro che definito, ma anzi aperto $e$ in continua evoluzione. La pubblicazione non del testo originario, ma di una versione "riveduta ed aggiornata", mi ha dato ragione e mi fa pensare che, pur avendo lasciato la stessa struttura della tesi di 
dottorato, Alessandra abbia voluto, da un lato, snellire i ragionamenti iniziali e, dall'altro, precisare la finalità/obiettivo finale del suo lavoro, mettendo a frutto il processo di formazione post dottorato attuato con la partecipazione a varie iniziative nazionali promosse dentro e fuori della nostra disciplina. Mi riferisco, in particolare, alla presentazione di un paper alla VII Conferenza Nazionale della Società degli Urbanisti Italiani (SIU) di Trento e alla sua partecipazione al Corso di approfondimento sul governo del paesaggio presso la Fondazione Benetton nel 2004 su "Nella città diffusa. Idee, indagini, proposte per la nebulosa insediativa veneta".

Due occasioni in cui questo lavoro iniziato con la ricerca di dottorato ha avuto modo di essere promosso come tema di confronto pubblico fra urbanisti (SIU), ma anche di porsi come occasione di discussione-riflessione interdisciplinare (Fondazione Benetton).

Un'ultima battuta vorrei farla per sottolineare la validità delle iniziative del Dottorato in Progettazione Paesistica dell'Università di Firenze che promuove durante il triennio occasioni di confronto pubblico per i dottorandi, e, a tesi conclusa, opportunità per pubblicizzare i risultati raggiunti dai suoi dottori, presentandoli al mondo accademico in maniera semplice, ma proficua.

Antonella Galassi

Facoltà di Architettura - Università di Roma "La Sapienza" 


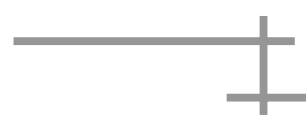

Premessa 


\section{LO SCENARIO DI PARTENZA}

Nei documenti preparatori della $1^{\underline{a}}$ Conferenza Nazionale per il Paesaggio Vittoria Calzolari ${ }^{1}$ definisce il paesaggio come

la manifestazione sensibile e percepita in senso estetico del sistema di relazioni che si determina nell'ambiente biofisico e antropico e che caratterizza il rapporto delle società umane e dei singoli individui con l'ambiente e con il territorio, con i siti e i luoghi, in cui si sono sviluppati, abitano e operano.

Di tale sistema, secondo questa chiave di lettura, fanno parte essenzialmente due ordini di elementi:

- le risorse primarie (terra, acqua, sole, aria) che, in relazione alle situazioni di altitudine, latitudine e allo spessore e fertilità del suolo, consentono e favoriscono tipi di flora e di associazioni vegetali, tipi di fauna e animali (tra cui l'uomo, visto come parte del regno animale). Questo insieme costituisce la struttura ambientale e le sue componenti sono studiate prevalentemente, singolarmente e nel loro insieme, dalle discipline fisico-naturalistiche.

- La popolazione umana, intesa come complesso di esseri pensanti che, nei diversi periodi storici e nelle diverse parti del globo terrestre si organizza e si insedia in modi diversi in rapporto alla propria identità e alla propria cultura (livelli sociali, economici, educativi, politici) e che prende coscienza e possesso dell'ambiente trasformandolo in territorio. Lo studio di

1 Contributo di Vittoria CALzOLARI, in Ministero per i Beni e le Attività Culturali, «Conferenza Nazionale per il Paesaggio - Lavori preparatori», Gangemi, Roma 2001, pp.56-58. 
questi caratteri e vicende è in gran parte compito delle discipline umanistiche.

Non è scopo di questo lavoro ripercorrere le vicende che nel nostro paese hanno visto mutare, anche profondamente, la concezione di paesaggio, ma è sicuramente utile ricordare brevemente come la ricerca del sistema di relazioni sia oggi diventata il comune interesse e il terreno di incontro e di colloquio tra settori disciplinari diversi, che fin dall'inizio del XX secolo si erano separati, contrapponendosi su due fronti principali: quello umanistico - estetico - idealista da una parte, quello scientifico positivista dall'altra.

Per il giudizio estetico il paesaggio è considerato un'entità quasi priva di materia, forse più un punto di vista che un territorio, quasi una cartolina, un quadro, un panorama; scriveva a questo proposito Rosario Assunto:

vi sono paesaggi il cui essere estetico deriva non da un processo produttivo, ma da quello che si potrebbe chiamare un conferimento di senso rispetto al quale il loro essere materiale era preesistente: da una scoperta [...] per effetto della quale diventano oggetti estetici quelli che prima erano pure e semplici cose di natura ${ }^{2}$.

II paesaggio geografico entro il pensiero positivista, al contrario, secondo la definizione di Renato Biasutti, viene considerato

una sintesi astratta di quelli visibili ${ }^{3}$ in quanto tende a rilevare da essi gli elementi o caratteri che presentano le più frequenti ripetizioni sopra uno spazio

2 R. Assunto, Il paesaggio e l'estetica, Giannini, Napoli 1973; nuova ed. Novecento, Palermo 1994.

3 Ovvero quei paesaggi che l'occhio può abbracciare in un giro di orizzonte. 
più o meno grande, superiore, in ogni caso, a quello compreso da un singolo orizzonte.

II concetto di paesaggio - come già detto - è oggi notevolmente maturato grazie soprattutto all'interrelazione tra le diverse discipline che se ne occupano. Le nuove chiavi di lettura sono emerse sia durante la Conferenza Nazionale per il Paesaggio che si è tenuta alla fine del 1999 a Roma, sia attraverso l'orientamento offerto da due importanti documenti di livello nazionale ed internazionale.

Il primo, la Convenzione Europea del Paesaggio firmata a Firenze dagli Stati membri dell'Unione Europea il 20 ottobre 2000, si riferisce al paesaggio come elemento ambientale complesso, che svolge funzioni culturali, ecologiche, sociali ed economiche ${ }^{4}$.

II secondo, la cosiddetta Carta di Napoli, considera il paesaggio

come un sistema vivente in continua evoluzione che alle diverse scale:

- ha una forma fisica e un'organizzazione spaziale specifica (struttura)

- possiede una dinamica interna dovuta al movimento e al flusso di energia tramite acqua, vento, piante e animali (funzionamento)

- è soggetto ad evoluzione nel tempo in funzione della dinamica e delle modifiche nella struttura (cambiamento) ${ }^{5}$.

È dunque evidente come gli aspetti definitori del concetto di paesaggio siano stati oggetto di molteplici studi, soprattutto nella

4 Nella Convenzione europea del paesaggio, all'articolo 5 "Misure generali", si dice che: "Ciascuno Stato si impegna [...] a riconoscere giuridicamente il paesaggio in quanto componente essenziale dell'ambiente di vita delle popolazioni, espressione delle diversità del loro comune patrimonio culturale e naturale, e fondamento della loro identità".

5 Carta di Napoli. Il parere degli specialisti sulla riforma degli ordinamenti di tutela del paesaggio in Italia, Napoli 1999, punto 1, Contenuti e metodi per interpretare il paesaggio, p.3. 
seconda metà del $X X$ secolo. Ho già detto che non appartiene all'economia di questo lavoro riprenderli tutti, ma al fine di evitare ulteriori equivoci che ad oggi persistono, è opportuno specificare come questa ricerca faccia espressamente riferimento ad una visione sistemica (strutturale) del paesaggio, intendendolo essenzialmente come una serie di sistemi di elementi naturali ed artificiali (creati dall'uomo), interrelati, che si sono evoluti nei vari periodi storici e che, a seconda dell'interpretazione che di volta in volta l'uomo ne ha dato, hanno fatto sì che vi fossero paesaggi tanto naturali quanto artificiali di diversa forma e natura. Viene quindi fatto esplicito riferimento ad un concetto di paesaggio inteso come l'immagine delle relazioni di diverso tipo che si instaurano tra componenti morfologiche fisiche, naturalistiche, antropiche insediative storiche 0 meno, osservate secondo una logica di struttura.

Esso è dunque caratterizzato da un'articolazione strutturale e da processi funzionali ed evolutivi di tipo dinamico ${ }^{6}$.

In un'ottica di questo genere, la ricerca pone la propria attenzione sul paesaggio agrario $^{7}$ assunto come chiave di lettura privilegiata per interpretare ed analizzare gli assetti paesistici di una determinata porzione di territorio. II tema di studio viene dunque affrontato a partire dalla definizione degli

6 G. PAOLINELLI, La frammentazione del paesaggio periurbano. Criteri progettuali per la riqualificazione della piana di Firenze, Tesi del Dottorato di ricerca in Progettazione Paesistica, Firenze 2001, p.14.

7 Emilio Sereni ha definito il paesaggio agrario come:

quella forma che l'uomo, nel corso ed ai fini delle sue attività produttive agricole, coscientemente e sistematicamente imprime al paesaggio naturale

(E. SERENI, Storia del paesaggio agrario italiano, Laterza, Roma-Bari 1961; nuova ed. 1999, p.29). 
elementi componenti il paesaggio agrario, attraverso l'interpretazione delle permanenze e delle principali trasformazioni da esso subìte, e si pone come obiettivo generale la definizione di possibili criteri di progettazione paesistica compatibili e congruenti con la struttura attuale propria del paesaggio oggetto dell'applicazione pratica.

Le considerazioni metodologiche e l'applicazione operativa sono state possibili soprattutto grazie alla formazione di un supporto scientifico basato su una bibliografia di base ragionata (attraverso la quale è stato possibile individuare lo scenario di partenza e costruire la metodologia di ricerca) e specifiche bibliografie e cartografie riferite all'ambito oggetto di studio, che hanno permesso l'applicazione pratica della metodologia generale individuata.

L'attenzione sul paesaggio agrario nasce dal fatto che, più di qualunque altro, esso è stato frutto dell'azione continua dell'uomo, il quale è andato a modificare l'assetto fisico ed infrastrutturale del territorio cercando di adattarlo ai propri bisogni. I differenti assetti colturali, morfologici, insediativi, nascevano e tuttora nascono a seguito delle diverse conformazioni dei luoghi e delle diverse esigenze della popolazione.

II paesaggio agrario ha visto nel '900 modificarsi profondamente la propria struttura e i suoi processi formativi: prima in maniera lenta e più attenta alla natura dei luoghi, poi in modo sempre più violento e repentino, man mano che veniva ritenuto ambito marginale e residuale, senza un particolare valore ed interesse dal punto di vista paesistico.

Nell'ultimo decennio, però, si sta assistendo ad una sorta di inversione di tendenza. È stato infatti riconosciuto al paesaggio 
agrario un importante ruolo strategico nella politica di riqualificazione paesistica intrapresa dal nostro paese, fino ad arrivare a comprendere come buona parte del processo di riqualificazione dell'ambiente possa essere attuata innanzitutto attraverso un rilancio delle aree rurali di tipo tradizionale per la produzione di risorse paesistiche e una regolamentazione specifica finalizzata al controllo delle attività agricole moderne maggiormente produttive.

Nonostante questo rinnovato interesse, è tuttora riscontrabile una carenza di metodi e tecniche che presentino una qualche originalità circa l'analisi dell'organizzazione del territorio agricolo. Quand'anche questi metodi vengono proposti, le categorie concettuali secondo le quali viene affrontato il tema sono ancora in gran parte dei casi legate alla pianificazione tradizionale costruita in funzione delle esigenze dello sviluppo urbano ${ }^{8}, 0$, in altri lavori, sono fortemente dipendenti dalla storia del settore disciplinare entro il quale vengono definite.

A questo proposito già Lucio Gambi nel 1961 nel suo "Critica ai concetti geografici di paesaggio umano" rilevava come le tipologie paesistiche rurali enunciate dagli studiosi della geografia umana si rifacessero principalmente a quelle delineate da Bloch nel 1931 - ovvero l'«openfield» o paesaggio dei campi aperti, il «bocage» o paesaggio dei campi chiusi, e le «colture promiscue» - rimanendo

8 Tra i lavori svolti in Italia che si discostano da questa tendenza sono stati studiati soprattutto quelli curati dal prof. Pompeo Fabbri del Politecnico di Torino (il suo testo più famoso è senz'altro FABBRI P., Natura e cultura del paesaggio agrario: indirizzi per la tutela e la progettazione, Città Studi, Milano 1997) e quelli affrontati dall'Istituto di Architettura e Urbanistica dell'Università di Bologna a cura dei proff. Carlo Monti, Alberto Pratelli, Piero Secondini e del dott. Gabriele Riguzzi (gran parte della loro ricerca è descritta nel libro C. Montı, A. Pratelli, G. RiguzZI, P. SECONDINI, Analisi e pianificazione del territorio rurale, Clueb, Bologna 1985). 
ancorati ad una metodologia descrittiva delle strutture agrarie, entro un'interpretazione dell'organizzazione dello spazio rurale come adeguamento alla funzionalità biologica delle coltivazioni ${ }^{9}$.

In questo lavoro si è dunque cercato di costruire un metodo di lettura che fosse innanzitutto:

- comprensivo, e non settoriale, in maniera tale da esaminare le relazioni dinamiche fra le componenti il sistema «paesaggio agrario», piuttosto che le singole componenti in sé;

- progettuale, e non meramente descrittivo, finalizzato cioè all'individuazione degli elementi strutturanti di ogni tipologia di paesaggio agrario, delle cosiddette "invarianti" che si sono storicamente poste come matrici (segni), dei diversi gradi di adattamento delle diverse componenti.

I principali passaggi adottati sono stati:

a. riconoscere la struttura del sistema «paesaggio agrario» attraverso l'individuazione dei suoi elementi componenti e delle relazioni che fra essi si instaurano, al fine di interpretare alcune delle principali tipologie di paesaggio agrario italiane [Cfr. capitolo 1];

b. ripercorrere i principali cambiamenti subìti nell'ultimo secolo dal sistema «paesaggio agrario», identificando quelle che ne sono state le cause principali ed evidenziando i nuovi processi in atto che hanno portato il paesaggio agrario ad essere considerato elemento di connessione delle emergenze ambientali e paesistiche e presidio per il territorio [Cfr. capitoli 2 e 3];

9 L. GAMBI, Critica ai concetti geografici di paesaggio umano, Lega, Faenza 1961. 
c. comprendere il funzionamento del sistema «paesaggio agrario» attraverso il riconoscimento e lo studio dei principali e più significativi indicatori del suo stato di salute [Cfr. capitolo 4].

La ricerca è, secondo quanto detto poc'anzi, fondamentalmente di tipo applicativo.

Essa è basata su una serie di osservazioni fatte sul paesaggio agrario dell'area romana, riconoscendo a questa porzione di territorio una forte connotazione paesistica e attribuendole il ruolo di importante risorsa sia per la sua estensione, sia per i suoi caratteri specifici.

Piuttosto che ad una lettura geografica di tale contesto, condotta per tipologie territoriali omogenee dal punto di vista morfologico ed ambientale, nel lavoro si è proceduto ad una lettura di tipo sistemico/strutturale, legata al riconoscimento degli elementi componenti la struttura agraria e finalizzata all'individuazione e all'interpretazione delle interrelazioni che danno origine alle diverse tipologie di paesaggi agrari, secondo le quali è possibile articolare oggi la "campagna romana" in "campagne romane".

Si è quindi proceduto alla costruzione di una quadro sinottico nel quale far risaltare le differenze fra le varie "campagne romane", cercando di mettere in evidenza come in ciascuna attualmente convivano quell'insieme di caratteri e segni che hanno dato origine ad una certa struttura tradizionale di paesaggio agrario (permanenze e persistenze), insieme a tutta una serie di nuovi elementi (trasformazioni) che costituiscono, in alcuni casi, dissonanze e lacerazioni rispetto alla struttura 
tradizionale e, in altri, nuove e riconosciute componenti di una nuova connotazione paesistica.

Lo scopo principale al quale vuole giungere la ricerca è quello di definire alcuni possibili criteri di progettazione paesistica che siano congruenti con la struttura attuale di ciascuna "campagna romana" e che traggano la loro origine e definizione dall'interpretazione tanto degli elementi tradizionali ancora riconoscibili, quanto del risultato delle principali trasformazioni subìte. Ciò che si vuole raggiungere è una salvaguardia dell'integrità delle antiche strutture agrarie insieme al riconoscimento del valore ambientale e paesistico di quelle che oggi rappresentano nuove configurazioni agricole di pregio.

I principali passaggi dell'applicazione pratica sono stati:

d. individuare una possibile delimitazione del campo applicativo soprattutto attraverso l'analisi di quanto già presentato da studi e ricerche condotti sullo stesso ambito di studio [Cfr. capitolo 5];

e. identificare le cause e, soprattutto, gli effetti delle principali trasformazioni subìte dalle «campagne romane» attraverso l'interpretazione dei caratteri e dei segni propri delle strutture tradizionali (permanenze e persistenze) e degli elementi (trasformazioni) propri delle nuove connotazioni paesistiche. [Cfr. capitolo 6];

f. articolare la «campagna romana» in "campagne romane» attraverso il riconoscimento degli elementi componenti ciascuna struttura agraria e finalizzata all'individuazione e all'interpretazione delle reciproche interrelazioni che danno origine alle diverse tipologie di paesaggi agrari [Cfr. capitolo 7; 
g. definire alcuni possibili criteri di progettazione paesistica che siano congruenti con la struttura attuale di ciascuna «Campagna romana» e che traggano origine dalla comprensione di come sono cambiate le interrelazioni che si instaurano tra i diversi elementi componenti e che danno origine a nuovi quadri paesaggistici. 


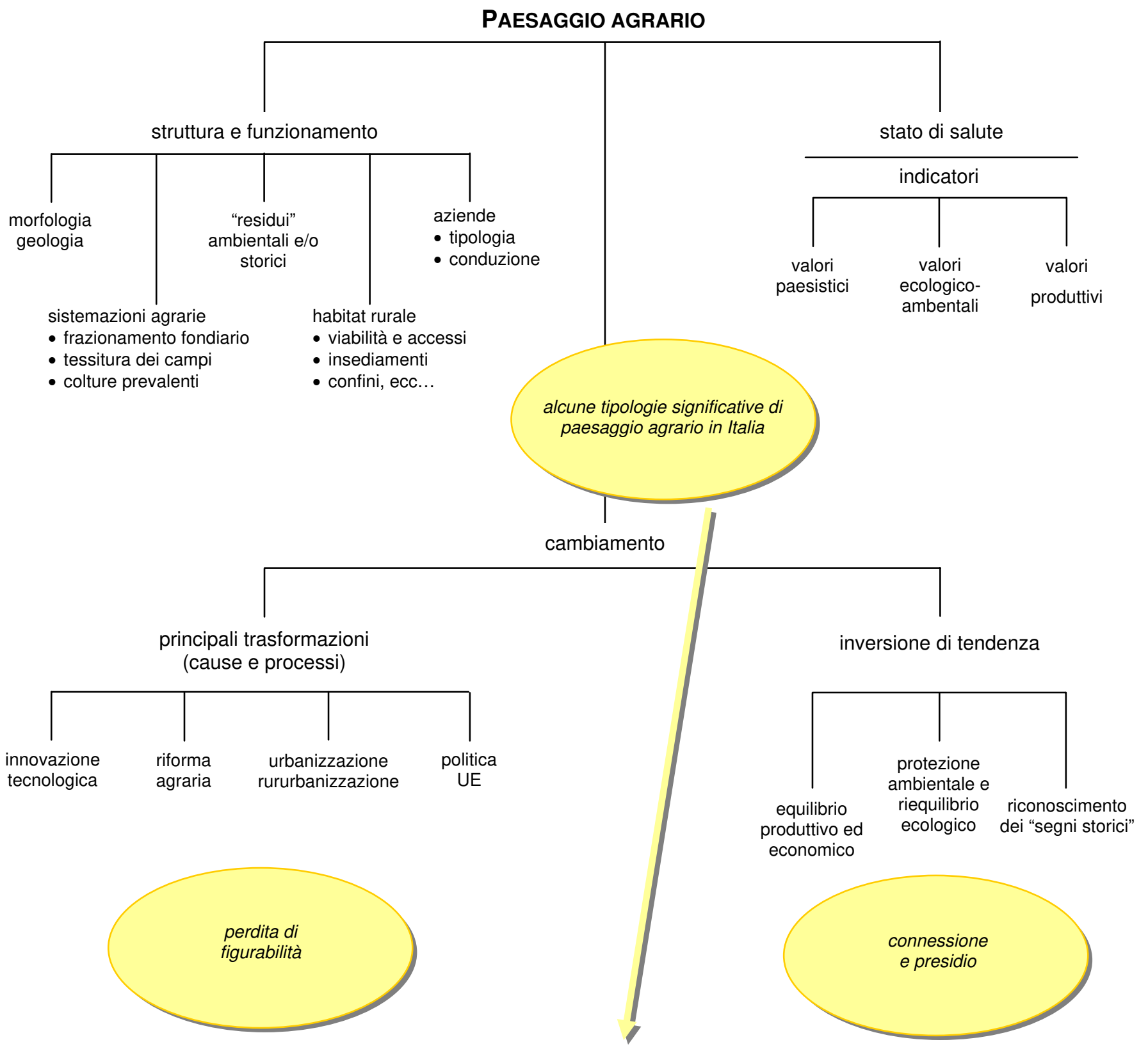

Campagna Romana

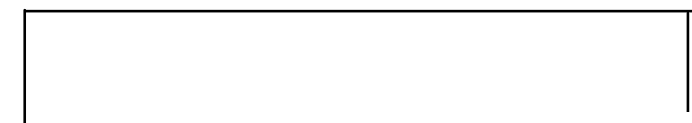

trasformazioni

(cause ed effetti)

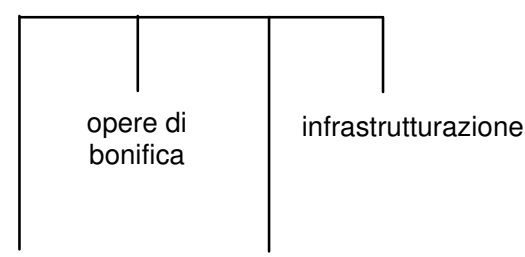

proprietà urbanizzazione

rendita possibili tipologie di

"campagne romane"

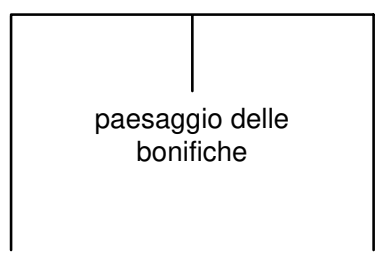

paesaggio delle grandi tenute paesaggio della

città 


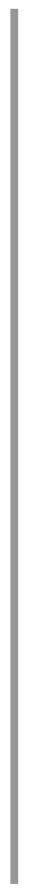

\section{PARTE PRIMA}

UNA POSSIBILE METODOLOGIA PER LA LETTURA DEL

PAESAGGIO AGRARIO

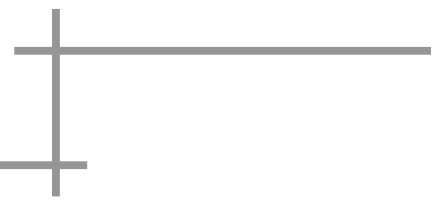


Il filo conduttore (...) è il concetto di sistema, riferito a diversi ambiti, oggetti e scale. Da questa linea di ricerca deriva la tendenza a individuare e interpretare le strutture piuttosto che i singoli oggetti e, all'interno delle strutture, le correlazioni tra le componenti fisico-naturalistiche e antropiche $(\ldots){ }^{1}$

\section{LA STRUTTURA DEL PAESAGGIO AGRARIO: GLI ELEMENTI COMPONENTI IL PAESAGGIO AGRARIO E LE LORO RELAZIONI}

Il paesaggio agrario - come si è detto - è frutto dell'azione continua dell'uomo, che ha modificato il territorio nel suo assetto fisico ed infrastrutturale per adattarlo, in ogni tempo e modo, alle proprie esigenze, legate in primo luogo ai bisogni alimentari'. Nelle diverse fasi storiche le diverse esigenze dettate dai mutamenti di ordine sociale, tecnologico, economico, e la conformazione dei luoghi che via via si conquistavano, hanno prodotto di volta in volta assetti paesistici diversi, caratterizzati ciascuno da una diversa combinazione di elementi colturali, irrigui, morfologici, insediativi.

1 V. Calzolari, Beni culturali e valori storico-paesistici delle aree protette di RomaNatura, in «Urbanistica Quaderni» n.37, mar., INU edizioni, Roma 2003, p.32.

2 Emilio Sereni ha definito il paesaggio agrario come

quella forma che l'uomo, nel corso ed ai fini delle sue attività produttive agricole, coscientemente e sistematicamente imprime al paesaggio naturale

(E. SERENI, Storia del paesaggio agrario italiano, Laterza, Roma-Bari 1961; nuova ed. 1999, p.29). 
Per poter affrontare un discorso compiuto sul paesaggio agrario attuale e sulle trasformazioni che lo hanno modificato soprattutto negli ultimi decenni, è essenziale individuare un paradigma di lettura adatto e, vista anche l'economia generale di questo lavoro, non troppo dispersivo.

La forma del paesaggio è diretta conseguenza delle forze agenti su di esso: forze che, se in apparenza sembrano indipendenti, sono in realtà strettamente dipendenti l'una dall'altra. Ė importante, quindi, non solo analizzare ogni singola componente, ma anche, e specialmente, tutti i possibili elementi di connessione fra le varie forze $e^{3}$.

Lo scopo principale vuole essere quello di risalire alla sostanza delle forme che caratterizzano il paesaggio agrario e alla complessa interrelazione che avviene tra $i$ suoi elementi componenti e che, ogni volta, dà origine a diverse strutture agrarie.

$\mathrm{Nel}$ panorama scientifico italiano ed europeo approcci al paesaggio di questo tipo sono stati condotti in diversi settori tematici, ciascuno con scopi ed obiettivi specifici.

P. George ${ }^{4}$, ad esempio, indicava già nel 1965 un'importante prospettiva di metodo circa i criteri di lettura del territorio rurale, individuando nelle caratteristiche dell'uso del suolo, nella struttura della proprietà fondiaria e nella dimensione media dei campi coltivati, alcuni elementi rilevatori della tipologia agraria prevalente in una determinata zona, intendendo questa come frutto dell'intreccio - storico - che avviene tra fattori socio-economici e fattori fisici locali.

3 L. Carbonara, I paesaggi naturali: analisi e pianificazione, in «Rassegna di architettura e urbanistica» nn.47-48, ago.-dic., Kappa, Roma 1980, p.150.

4 P. George, Manuale di geografia rurale, Edizioni di Comunità, Milano 1972. 
Altri studiosi, di diverso orientamento ed ambiente culturale, hanno in modo analogo inteso la conoscenza del territorio rurale come individuazione degli elementi tipologici caratterizzanti e delle relazioni che tra essi si instaurano, organizzando e trasformando il paesaggio. Emilio Sereni $i^{5}$ e Renato Zangheri ${ }^{6}$, ad esempio, hanno identificato, in diversi loro studi sull'ambiente rurale, la struttura della proprietà come elemento cardine dell'organizzazione dell'insediamento; così come in altre ricerche altri autori hanno considerato le diversità nelle tecniche di coltivazione ed appoderamento, leggibili sul territorio, ed ancora più le trasformazioni che essi hanno subito, come elementi esplicativi della natura dei processi di evoluzione dell'organizzazione sociale delle campagne.

La ricerca, partendo e prendendo spunto da questi studi, in questo capitolo intende arrivare ad una interpretazione del paesaggio agrario definendo quali sono gli elementi componenti attraverso $i$ quali riconoscerne la struttura ${ }^{8}, e$

\footnotetext{
5 E. SERENI, op.cit.
}

6 R. ZANGHERI, Agricoltura e contadini nella storia d'Italia, Einaudi, Torino 1977.

7 M. BLOCH., Les caractères originaux de l'histoire rurale française, Parigi 1953; D. FAUCHER, Géographie agraire, Parigi 1949.

8 Riconosciuto che il concetto di paesaggio in Italia ha attualmente molteplici interpretazioni e che in questo documento si fa esplicito riferimento a quella che considera il paesaggio come "un sistema vivente in continua evoluzione" che alle diverse scale:

- ha una forma fisica e un'organizzazione spaziale specifica (struttura)

- possiede una dinamica interna dovuta al movimento e al flusso di energia tramite acqua, vento, piante e animali (funzionamento)

- è soggetto ad evoluzione nel tempo in funzione della dinamica e delle modifiche nella struttura (cambiamento).

(Carta di Napoli. Il parere degli specialisti sulla riforma degli ordinamenti di tutela del paesaggio in Italia, Napoli 1999, punto 1. Contenuti e metodi per interpretare il paesaggio, p.3). 
leggendo l'organizzazione spaziale specifica che nel tempo è mutata, in alcuni casi approfondendosi e in altri semplificandosi.

$\mathrm{Dal}$ punto di vista specifico assunto, tale lettura avviene attraverso l'uso di alcuni parametri ritenuti elementi strutturanti:

- le sistemazioni agrarie, intendendo con questo termine ciò che riguarda il frazionamento fondiario, l'andamento morfologico dei terreni, la tessitura delle coltivazioni e le colture prevalenti;

- i "residui" ambientali e/o storici;

- l'insediamento rurale, ovvero i dati pertinenti al sistema degli accessi, della viabilità e dei percorsi poderali, alla posizione del casale e degli annessi agricoli, alle modalità con cui vengono costruiti i confini, ecc...;

- la tipologia delle aziende e della loro conduzione. 


\section{LE SISTEMAZIONI AGRARIE}

L'occupazione del territorio da parte dell'uomo per l'attività agricola ha generato una serie di segni paesaggisticamente di grande rilevanza in quanto ricchi di un notevole valore informativo. Ne fanno parte quelli propri della trama di appoderamento e quelli dell'ordinamento colturale ${ }^{9}$.

La trama di appoderamento può essere considerata come la visualizzazione sul territorio del disegno della maglia catastale. Tale visualizzazione può avvenire in maniera molto varia e può essere evidenziata da un semplice cambio di tipo di colture, così come dalla presenza di elementi divisori (muretti a secco, siepi, ecc...) o di altre strutture più complesse (i canali di irrigazione, ...).

L'esistenza o meno di questo tipo di elementi ha portato alla fondamentale distinzione fra due tipologie prevalenti di disegni agricoli: i campi chiusi e i campi aperti ${ }^{10}$.

I primi, i campi chiusi, presentano una trama di appoderamento più minuta, sottolineata dalla presenza di un gran numero di recinzioni e alla cui scala si proporzionano tutti gli altri elementi (insediamenti, percorsi, accessori, ecc...). Questo tipo di configurazione

trova la sua origine più importante nel senso di proprietà e nella necessità di una difesa di questa ${ }^{11}$;

la ritroviamo nella colonizzazione agricola romana della centuriatio e, soprattutto, nella configurazione dell'hortus conclusus nato anch'esso in

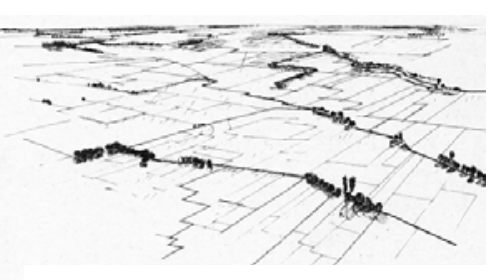

Figura 1. Campi aperti. epoca romana, poi presente nelle città del Medioevo e del Rinascimento. L'iniziativa individuale e il senso di proprietà sono gli elementi che storicamente si possono indicare come "input" per la

P. FABBRI, Natura e cultura del paesaggio agrario: indirizzi per la tutela e la progettazione, Città Studi, Milano 1997, pp.106-107.

10 Allo stesso modo i francesi distinguono fra i bocages e le campagnes e gli inglesi fra gli enclos e gli openfields.

11 G. FerRara, L'architettura del paesaggio italiano, Marsilio, Padova 1968, p.82. 
formazione di questo tipo di appoderamento, che viene generalmente caratterizzato non solo e non tanto da un tipo particolare di coltura, quanto dal disegno dei confini dei singoli appezzamenti, costruiti o con muri di pietra, o con siepi vive, oppure semplicemente con canali o con piccoli fossi di scolo.

I campi aperti, anche se non è sempre possibile e corretto generalizzare, si distinguono dai precedenti per la mancanza di delimitazioni e per una scala diversa, caratterizzata da una particolare ampiezza. Le concezioni che hanno dato origine, sia in diverse fasi storiche sia in diverse zone geografiche, a questo tipo di trama sono state fondamentalmente due.

Una prima forma di paesaggio a campi aperti deriva da un uso collettivo dello spazio: l'ager publicus della civiltà romana, che comprendeva tutte le terre che non venivano suddivise fra i cittadini, i boschi e soprattutto i pascoli, ne è un esempio. Oggi in alcune zone si assiste ad una simile figura di campi aperti quando negli sfruttamenti agrari è previsto un uso del suolo contemporaneamente pubblico e privato (cito ad esempio certe aree del Trentino, nelle quali c'è l'usanza che tutti i componenti la comunità rurale svolgano insieme le principali attività agricole della semina, del raccolto e, infine, della vendemmia e della mietitura).

Una seconda concezione di paesaggio a campi aperti, radicalmente opposta, ha origine durante il periodo feudale, in cui si afferma il regime delle concessioni; essa è dovuta all'accentramento di grandi estensioni di terreno in poche mani: il «latifondo».

La differenziazione morfologica che intercorre tra le due tipologie di paesaggio a campi aperti - da una parte la "veste nuova" dell'ager publicus romano e dall'altra il latifondo - consiste soprattutto, ma non solo, in una diversità di scala. Nelle aree in cui prevale la "concezione collettivistica" il rapporto fra uomo e ambiente è molto stretto e l'immagine paesistica dei luoghi è data dalla presenza di un'architettura 
rustica sparsa e ricorrente, di ampi campi suddivisi tra loro da vaste zone di bosco e di colture viticole e frutticole specializzate. Tali elementi si relazionano in maniera tale da dare origine, pur nella vastità degli orizzonti, a tante scene differenti. Nel latifondo, al contrario, il territorio ha un ritmo sempre uguale nel quale il più delle volte si ha solo l'alternanza tra il seminativo e i prati incolti, e rari boschi e centri rurali, che essendo molto distanti l'uno dall'altro rendono particolarmente difficili le comunicazioni.

Altro segno importante che caratterizza diverse tipologie di paesaggio agrario sono gli ordinamenti colturali, ovvero le modalità con le quali vengono disposte le coltivazioni (cereali, vigneti, frutteti, ecc...). Essi possono essere suddivisi principalmente in due tipologie, ciascuna delle quali dipende essenzialmente dalla morfologia dei terreni sui quali ci si trova ad operare: vi sono, infatti, quelli che disegnano i terreni in pianura e quelli che riguardano $i$ terreni collinari e pedemontani. La diversità negli ordinamenti dipende, inoltre, dalle diverse sistemazioni idrauliche che vengono effettuate sia per facilitare le operazioni di coltivazione, sia soprattutto per controllare il regime delle acque - in eccesso e in difetto - di superficie e in profondità.

Anche se a seguito della meccanizzazione dell'agricoltura il paesaggio agrario attuale si è omogeneizzato ${ }^{12}$ per rispondere meglio alle esigenze proprie della scala più ampia della macchina, può essere conservata una sintetica distinzione di massima tra l'organizzazione dei campi nei terreni di pianura e quella nei terreni in pendio.

Nei terreni di pianura, pur se la natura dei suoli e le ragioni storiche hanno fatto sì che ci siano sistemazioni idrauliche e ordinamenti colturali diversi, l'organizzazione è solitamente caratterizzata ${ }^{13}$ da una

\footnotetext{
12 Vedere su questo argomento e sulle principali cause che hanno portato alle profonde trasformazioni che ha subito il paesaggio agrario il capitolo successivo.

13 P. FABBRI, op. cit., pp.111-113.
} 
suddivisione degli appezzamenti più o meno regolare che corrisponde alle diverse proprietà fondiarie e che si appoggia alla rete stradale di accesso ai campi e al sistema dei fossi. Questi ultimi fungono sia da canali di irrigazione e di adduzione delle acque - soprattutto nelle pianure irrigue - sia da canali di raccolta dell'eccesso della pioggia.

Nei terreni in pendio, i principali tipi di sistemazione hanno in qualche modo cercato di correggere gli effetti negativi della pendenza del suolo: possono essere a terrazzo, a ciglioni e a gradoni.

Vi sono poi ordinamenti colturali propri dei terreni collinari, che non sono legati a particolari opere di sistemazioni idrauliche o di correzioni dell'acclività dei terreni: la sistemazione a cavalcapoggio, la sistemazione a girapoggio e la sistemazione a rittochino ${ }^{14}$.

Le trame di appoderamento e gli ordinamenti colturali appena descritti costituiscono lo "scheletro" del territorio, la sua griglia strutturale; le colture, poi, rivestono questa ossatura contribuendo a definire $\mathrm{i}$ diversi tipi di paesaggio agrario che cambiano anche a seconda del clima, delle condizioni economiche specifiche e della struttura oro - idro - geologica propria di ciascun luogo.

Gli esempi che si possono riportare sono certamente numerosi. Occorre citare quelli più significativi, attraverso i quali si possono interpretare le diverse conformazioni che assume il paesaggio agrario italiano $^{15}$.

14 Il cavalcapoggio è caratterizzato da campi a doppia pendenza, posizionati uno verso valle e uno nel verso opposto, e dalle fosse di regolamentazione delle acque che tagliano le curve trasversalmente, così come l'ordinamento delle colture; il girapoggio prevede un sistema di regimentazione delle acque ed un conseguente ordinamento colturale che segue le curve di livello; il rittochino consiste nella disposizione dei fossi di scolo a distanze più o meno ravvicinate lungo la pendenza massima e dei "capifossi" di traverso e con una pendenza ridotta.

15 Su questi argomenti, oltre a Guido FERRARA che li tratta brevemente nel suo libro L'architettura del paesaggio italiano, l'opera di maggior dettaglio è sicuramente quella di Emilio SERENI, che nella sua Storia del paesaggio agrario italiano (Laterza, RomaBari 1961; nuova ed. 1999) ricostruisce i lineamenti del paesaggio agrario italiano nei principali periodi della storia del nostro paese. 
Nel caso del seminativo semplice, il paesaggio presenta, pur costituendo un ambiente monotono, una certa mutevolezza stagionale per la caducità del manto vegetale. Questa coltura è caratteristica delle zone agrarie in cui prevale la trama dei campi aperti ed è solitamente sintomo della mancanza di alternativa: ai campi di grano si alternano infatti i campi ad erba senza alcuna soluzione di continuità.

Il seminativo arboreo è, rispetto al precedente, caratterizzato da una maggiore varietà: anche se non arriva ad avere un disegno così minuzioso come nel caso della trama dei campi chiusi, esso forma una scacchiera molto varia.

In questa fattispecie il terreno non è mai dedicato ad un solo tipo di produzione, ma al contrario è caratterizzato dall'alternanza fra colture differenti (cerealicole, foraggiere o arboree).

Le colture specializzate sono quelle dove, a differenza delle precedenti, la presenza di un tipo vegetale predomina largamente su tutti gli altri. L'esempio più caratteristico è quello della vite, che viene coltivata in modi anche molto diversi tra loro in molte zone del nostro paese e, proprio a seguito di questa diversità di coltivazione, aiuta a conformare l'ambiente in tutta una serie di tipi differenti ${ }^{16}$.

Un altro esempio di coltura specializzata, anch'essa particolarmente importante per comprendere le caratteristiche morfologiche del paesaggio in Italia, è l'olivo, il quale generalmente è disposto a filari equidistanti che seguono, accompagnandolo, l'andamento del terreno e che con la loro regolarità smorzano le differenze fra i vari luoghi, costituendo una presenza continua e unificante.

16 I vigneti siciliani e quelli del Salento, ad esempio, non presentano alcun tipo di sostegno e sono piantati ad "alberello"; un altro esempio è quello della pianura emiliana, dove la vite viene fatta sviluppare verticalmente, facendola appoggiare ad alberi di acero o di olmo; nelle zone montane della Valle d'Aosta o dell'Alto Adige, altro esempio ancora, per evitare il gelo e favorire l'irraggiamento solare la vite viene coltivata su vere e proprie pergole. 
Un'altra coltura specializzata è poi quella del riso ${ }^{17}$, anch'essa dotata di una forte carica espressiva. L'elemento che più la caratterizza è la geometria dei vasti acquitrini, con l'aspetto generalmente desolante di questi paesaggi: questa desolazione è dovuta anche ad una tangibile assenza della figura umana - che accede alle risaie solo al momento della piantata e a quello del raccolto - e all'organizzazione sociale che prevede l'uso di manodopera avventizia e salariata, portando quindi al diradamento delle costruzioni nel territorio.

\section{L'INSEDIAMENTO RURALE}

L'insediamento rurale è, in gran parte, quello che Marcello MAROcco chiama «habitat», definendolo come:

il modo di distribuzione della popolazione contadina, con le relative infrastrutture edilizie residenziali e produttive, nell'ambito di un dato territorio ${ }^{18}$.

Secondo Guido FERRARA l'habitat è un parametro analitico/valutativo fondamentale, in quanto

finisce col diventare una specie di cartina tornasole capace di mettere in evidenza se un paesaggio contiene dei valori o meno, se fa parte di un ordine mentale umano o se le sue forme sono casuali o preesistenti a questo, se è in grado di raccontare una storia o se è del tutto inespressivo sotto il profilo culturale ${ }^{19}$.

17 II riso fino agli anni '20 si ritrovava, in Italia soprattutto in Lombardia-Piemonte, nel Veneto e nell'area grossetana, coltivato in piccoli appezzamenti. Oggi, al contrario, la coltivazione è concentrata in grandi estensioni solo in qualche zona del nostro paese: nel mantovano, nell'Emilia e in Lombardia (Cfr. G. FERRARA, op. cit., p.130).

18 M. MAROCCO, II progetto dello spazio rurale, in «Rassegna di architettura e urbanistica» nn.47-48, ago.-dic., Kappa, Roma 1980, pp.83-84.

19 G. FERRARA, op. cit. 
In diversi contributi analitici recenti si assiste al tentativo di superare le "canoniche" categorie descrittive basate esclusivamente sulla classificazione tipologica degli edifici, ponendo invece l'attenzione sui caratteri formali dell'ambiente insediativo rurale. Nel complesso gli studi svolti ${ }^{20}$ hanno messo in relazione le regole morfologiche di costruzione dello spazio con i processi sociali, economici e tecnici ad esse sottese.

Le forme che hanno assunto gli insediamenti - forme che sono dipese tanto da condizionamenti fisici, quanto da condizionamenti storici, sociali, culturali ed economici - così come appaiono oggi sono il frutto di un lungo processo evolutivo. Tale processo si è basato sulla diversa relazione che si è via via formata fra alcuni elementi ricorrenti ${ }^{21} \mathrm{e}$ ha dato origine a forme assolutamente diverse tra loro a seconda, appunto, delle specifiche finalità, oltre che dei condizionamenti esterni.

Questi elementi, relativi essenzialmente ai caratteri generali propri dell'unità insediativa, sono:

- la dimensione dell'insediamento;

- il grado di compattezza;

- l'eventuale presenza di un tessuto e di un disegno di suolo;

- la forma della maglia viaria sulla quale l'insediamento si è sviluppato;

- l'eventuale presenza, all'interno dell'insediamento, di vuoti o di propaggini del territorio agricolo;

- il contesto fisico - ambientale entro il quale l'insediamento è inserito.

20 Molti studi hanno avuto come campo applicativo soprattutto la campagna veneta e le trasformazioni che il patrimonio insediativo di questa ha subito negli ultimi decenni.

Cfr. M. SARTORE, Forme e processi di urbanizzazione diffusa. Un'analisi della morfologia insediativa in un'area rurale del Veneto centrale, in "Archivio di studi urbani e regionali» n. 32, Franco Angeli, Milano 1988, pp.165-218; A. CECCHETTO, Archeologia rurale e variazioni tipologiche, in «Urbanistica» n. 86, marzo, Franco Angeli, Milano 1987, pp.10-19.

21 Cfr. P. FABBrI, op. cit., pp.144-164 nelle quali affronta il tema degli insediamenti abitativi nel paesaggio agrario, nonché le ricerche sui processi di urbanizzazione diffusa in Veneto condotte da M. SARTORE, op. cit., pp.165-218. 
Forme prevalentemente accentrate:

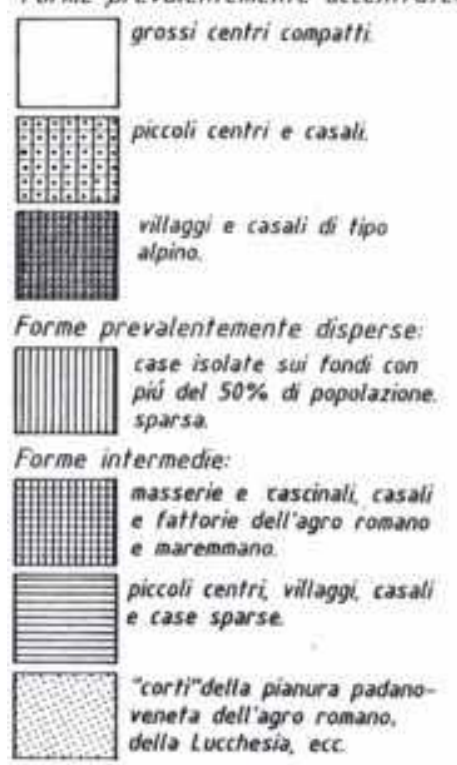

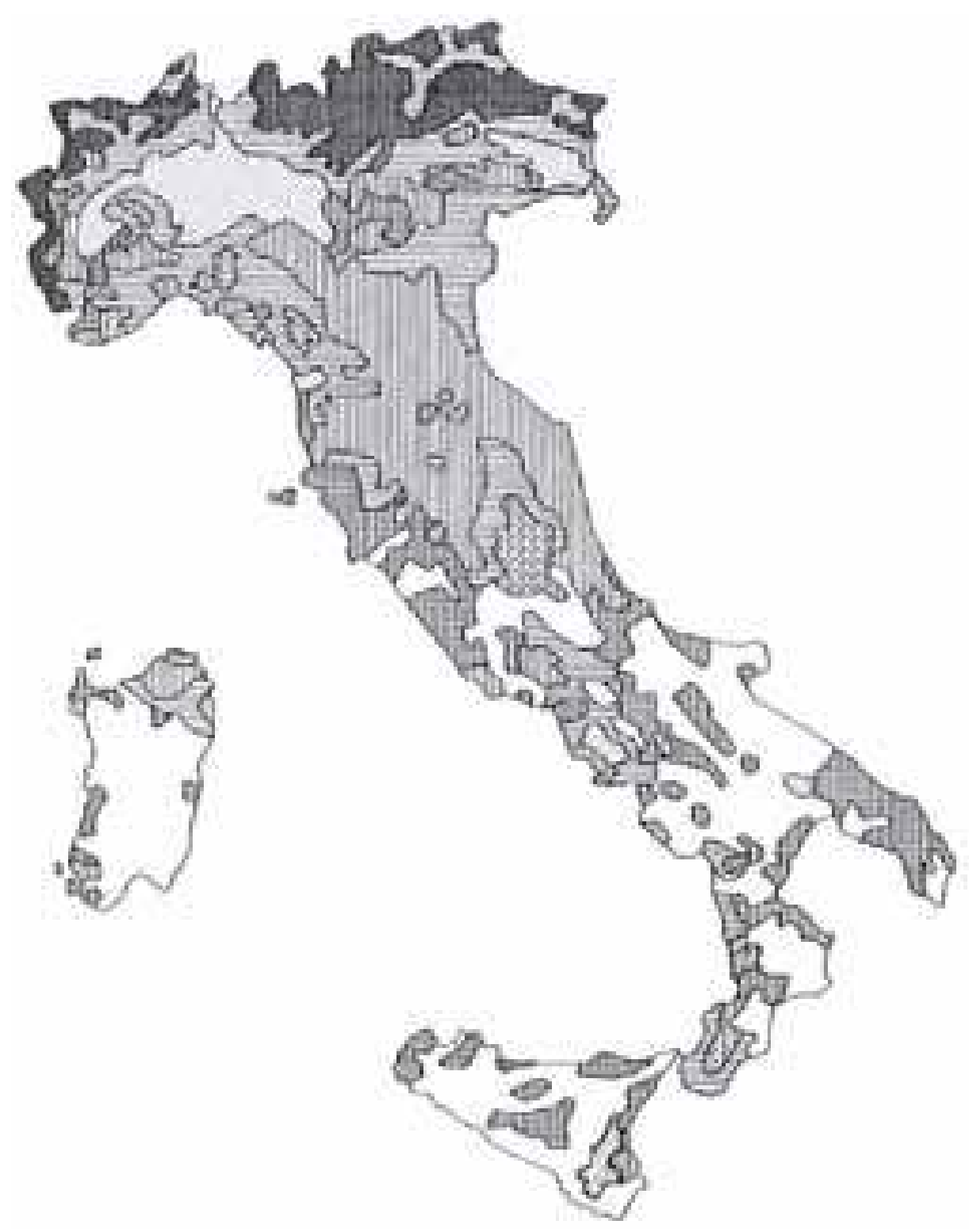

Figura 3. Le tipologie dell'insediamento rurale in Italia

Attraverso lo studio delle diverse relazioni che si instaurano tra gli elementi ora elencati, è possibile riconoscere, in maniera del tutto strumentale, alcune delle principali forme insediative che si possono ritrovare nel paesaggio agrario. 
Le case sparse sono quegli edifici che si ritrovano isolati nella campagna e distanti da altri edifici ${ }^{22}$ (masserie, casali, cascine, ...). II contesto ambientale è tipicamente agricolo e la suddivisione dei suoli corrisponde alla maglia poderale e all'orditura dei campi.

L'accesso agli edifici avviene direttamente da strade che sono in gran parte di interesse locale $\mathrm{o}$, più frequentemente, da strade bianche che fungono da collegamento fra la viabilità locale e i singoli

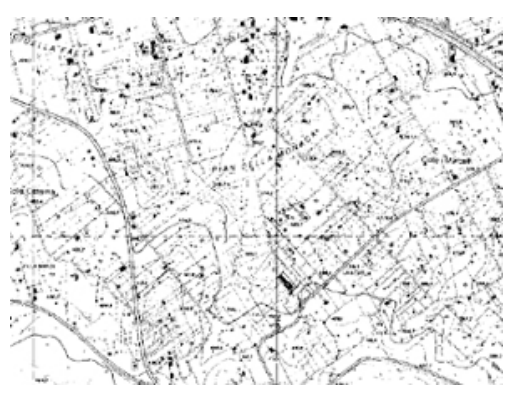

Figura 4. Case sparse. insediamenti e proseguono, talvolta, nei campi retrostanti l'edificio. In questi ultimi casi, ci si trova di fronte a strade che, oltre a connettere gli edifici con la viabilità pubblica, collegano anche quest'ultima - e gli stessi edifici - con i campi coltivati. In entrambi i casi, questo tipo di viabilità non attraversa quasi mai i fondi, "rompendo" il tessuto poderale, ma, al contrario, concorre a formarne ed assecondarne la trama.

Solitamente, infine, l'orientamento planimetrico degli edifici risulta guidato da quello assunto dai campi, tanto da non tener conto della provenienza della strada di accesso all'edificio.

L'edificato lungo la strada è costituito dalla presenza di più edifici posti vicini tra loro, che si sviluppano lungo infrastrutture viarie e che, per questo, assumono una forma lineare. La strada, a prescindere dalla sua importanza in termini gerarchici nel territorio, assume il ruolo di "elemento catalizzatore" dell'edificato.

Questa tipologia insediativa non è del tutto omogenea: è possibile, infatti, individuare due sue diverse configurazioni a seconda della scansione esistente tra gli edifici e dell'alternanza dei lotti edificati e dei campi agricoli lungo il fronte stradale. Tali forme insediative sono l'urbanizzato agricolo e i frazionamenti fronte strada.

22 Secondo O. MARINELLI - nel suo Atlante dei tipi geografici, pubblicazione a cura dell'Istituto Geografico Militare, Firenze 1949 - la casa sparsa agricola è da intendersi come

la forma più semplice di località abitata costituita dalla dimora di una famiglia agricola isolata, nel fondo che essa stessa coltiva e da cui trae i mezzi di sostentamento. 


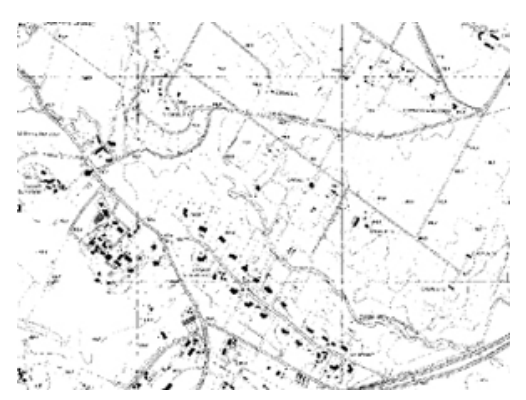

Figura 5. Urbanizzato agricolo.

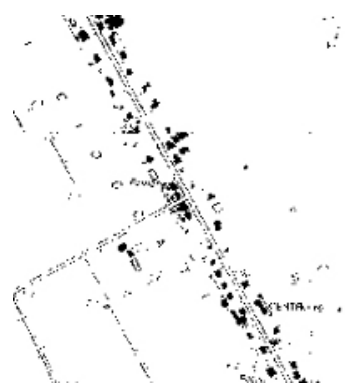

Figura 6. Frazionamenti fronte strada.
La prima - urbanizzato agricolo - è fortemente caratterizzata da aspetti di "ruralità", essendo storicamente legata a questo settore economico, e presenta una scarsa regolarità nella scansione tra gli edifici, di contro ad una forte regolarità nella successione di campi coltivati ed edifici.

Questi ultimi, infatti, non formano un continuum bensì si alternano a porzioni di spazi agricoli coltivati che giungono, nella maggior parte dei casi, a lambire la strada, costituendone praticamente la caratteristica preponderante.

II fabbricato, pur essendo prossimo alla strada, è decisamente arretrato rispetto a questa, formando in questo modo un ampio spazio antistante l'edificio che solitamente viene utilizzato come aia, cortile o orto. L'edificio è, poi, posizionato centralmente rispetto al "passo" del campo agricolo sul quale insiste e segue l'orditura dei campi, piuttosto che la giacitura della strada.

La seconda forma insediativa, quella dei frazionamenti fronte strada, si caratterizza, contrariamente al caso precedente, per la regolarità della scansione tra gli edifici e la sostanziale assenza di terreni agricoli tra i lotti edificati.

Ad un edificio si sussegue, lungo il fronte strada, un secondo edificio, poi un altro, e così via, e le distanze tra gli edifici sono molto simili. ${ }^{23}$

I frazionamenti appaiono, in molti casi, privi di alcun legame morfologico e formale con il territorio circostante; i lotti sui quali insistono gli edifici, poi, sono "chiusi" verso la campagna retrostante e non presentano nessun elemento di connessione con i campi coltivati ancora presenti alle loro spalle.

Se negli insediamenti del tipo precedentemente illustrato era soprattutto l'orditura del territorio agricolo a determinare l'orientamento planimetrico degli edifici, in questo caso, invece, questo è determinato

23 M. SARTORE, op. cit., p.193. 
dai lotti, che sono disposti planimetricamente in modo da assecondare l'andamento della strada - rispetto alla quale tendono a posizionarsi parallelamente - anche se questo va in molti casi ad interrompere e contrastare la continuità e "regolarità" del territorio agricolo.

L'esito spaziale di questa tipologia insediativa è quello di un continuum urbanizzato che presenta una forma abbastanza regolare e che appare come una sorta di cortina edificata che si frappone tra la strada e la campagna in maniera tale da non avere alcun contatto tra le due. I lotti sono in linea di massima di uguale dimensione, la tipologia edilizia è unica, con una copertura fondiaria che è all'incirca sempre la stessa.

Le micro-agglomerazioni rurali si possono considerare come insediamenti di modeste dimensioni inseriti in un ambito territoriale in cui prevalgono i caratteri agricoli, organizzati su una maglia stradale minima e caratterizzati da un tessuto che nella sostanza si presenta ordinato e compatto.

La concentrazione di più edifici (anche se solitamente non troppo numerosi) in ambiti caratterizzati da una bassa densità insediativa costituisce il carattere di più facile ed immediata differenziazione rispetto a tutte le altre forme insediative considerate sino ad ora.

Tale tipo di insediamento presenta una relativa omogeneità formale interna, accompagnata talvolta da una chiusura morfologica rispetto al paesaggio in cui è inserito e soprattutto rispetto ai suoi elementi formativi.

Queste caratteristiche predominanti rendono rappresentabile l'insediamento come un insieme sostanzialmente composito ma unitario, soggetto a tutta una serie di regole fisiche e formali del tutto differenti rispetto a quelle che regolano sia le case sparse, sia l'edificato fronte strada. Infatti, se nelle prime erano individuabili legami morfologici con l'ambiente circostante (ad esempio quelle tra l'orientamento dell'edificio 
e la trama del tessuto agricolo), e lo stesso, anche se in maniera più blanda, avveniva nel caso dell'edificato fronte strada (per ciò che riguardava l'orientamento dei lotti e la giacitura della strada), in questa tipologia l'individuazione di elementi fisici che fungano da legame tra la morfologia del paesaggio e quella dell'insediamento appare, generalmente, molto più difficile.

Solitamente le micro-agglomerazioni rurali non sono disposte lungo la viabilità pubblica e l'accesso a quest'ultima avviene attraverso strade bianche che, attraversando la campagna, raggiungono l'insediamento e spesso proseguono oltre, in maniera da fungere da collegamento sia tra la viabilità pubblica e il nucleo abitato, sia tra quest'ultimo e i fondi agricoli retrostanti.

Ad una prima lettura, la disposizione degli edifici all'interno dell'insediamento potrebbe apparire casuale 0 , comunque, priva di regole forti. Al contrario, attraverso un'osservazione più sistematica, si può riscontrare la ripetizione di alcuni "moduli di aggregazione". All'interno del costruito, infatti, è quasi sempre possibile individuare uno spazio sul quale prospettano tutti gli edifici e che si configura come il vero e proprio "centro" dell'insediamento. È su questo spazio che giunge la strada di accesso ed è a questo spazio comune che si connettono direttamente i singoli edifici.

\section{LA TIPOLOGIA DELLE AZIENDE E DELLA LORO CONDUZIONE}

L'ordinamento proprietario, organizzativo e di conduzione aziendale è stato in ogni tempo alla base di precisi assetti organizzativi del territorio e, quindi, del disegno del suolo agrario.

La cellula elementare dell'organizzazione agricola è l'azienda ${ }^{24}$.

24 Dal $3^{\circ}$ Censimento dell'agricoltura l'azienda viene definita come: "Unità tecnico economica costituita da terreni, anche in appezzamenti non contigui ed eventualmente impianti ed attrezzature varie, in cui si attua la produzione agraria, 
L'azienda può essere considerata sia dal punto di vista del titolo, per cui il terreno può essere in proprietà (o casi ad essa comparabili quali ad esempio l'usufrutto) 0 in affitto, sia dal punto di vista del tipo di conduzione, nel qual caso è possibile avere una conduzione diretta quando il conduttore presta egli stesso il lavoro manuale nell'azienda (anche quando sia coadiuvato da familiari o altri operatori esterni), oppure una conduzione con salariati quando il conduttore si avvale solo di manodopera esterna fissa o avventizia, o ancora una conduzione a colonia parziale appoderata (conosciuta di più con il termine "mezzadria") quando il conduttore si avvale di una persona che si impegna ad eseguire i lavori e a sostenere anche parte delle spese ${ }^{25}$.

L'immagine complessiva di una particolare tipologia di paesaggio agrario ha certamente subìto delle trasformazioni a seguito del modificarsi della struttura economica delle aziende, dell'evoluzione o involuzione del settore del quale queste si occupano, del mutare del tipo di produzione prevalente, ecc...

Gli elementi strutturali relativi alle aziende che permettono di ricostruire le diverse situazioni paesistiche possono essere sia di natura qualitativa - ovvero tutti i fenomeni più legati ai tipi di produzione - sia di tipo quantitativo - ad esempio le varie forme di conduzione o il numero e il taglio delle aziende stesse.

Dalla combinazione degli aspetti qualitativi e di quelli quantitativi, nonché dall'analisi di come la loro relazione si sia andata a modificare nel tempo con modalità e forme diverse, è possibile risalire ad alcune “immagini tipo" che, seppur in maniera un po' schematica, si possono riassumere in alcune casistiche presenti nello schema seguente.

forestale o zootecnica ad opera di un conduttore, e cioè persona fisica, società od ente che ne sopporta il rischio sia da solo, sia in associazione ad un mezzadro o colono parziario [...]".

Questa forma di conduzione è ora superata a seguito della cosiddetta legge sui fondi rustici, secondo la quale tale forma di accordo è trasformato in contratto d'affitto. 


\begin{tabular}{|c|c|}
\hline $\begin{array}{l}\text { TIPOLOGIA DI PAESAGGIO } \\
\text { AGRARIO }\end{array}$ & $\begin{array}{l}\text { TIPOLOGIA DI AZIENDE E } \\
\text { CONDUZIONE AZIENDALE }\end{array}$ \\
\hline $\begin{array}{l}\text { "paesaggio nudo" con } \\
\text { abbastanza ampi e regolari }\end{array}$ & $\begin{array}{l}\text { - presenza di grandi masserie } \\
\text { attrezzate sparse con coltivazioni } \\
\text { particolarmente produttive, quali ad } \\
\text { esempio i cereali, le piante industriali } \\
\text { o ortive a campo pieno, le colture } \\
\text { legnose specializzate } \\
\text { - conversione delle colture miste in } \\
\text { monocolture a causa della maggiore } \\
\text { produttività di queste e/o del } \\
\text { passaggio da una conduzione } \\
\text { familiare ad una salariata }\end{array}$ \\
\hline $\begin{array}{l}\text { paesaggio caratterizzato da una } \\
\text { notevole frammentazione dei fondi } \\
\text { agricoli }\end{array}$ & $\begin{array}{l}\text { proliferazione delle aziende a causa } \\
\text { della: } \\
\text { - marginalità e/o diseconomia del } \\
\text { sistema produttivo (improduttività) } \\
\text { - presenza di colture specializzate } \\
\text { (produttività) } \\
\text { - riconversione del sistema produttivo } \\
\text { (nel caso della conversione delle } \\
\text { zone del latifondo) }\end{array}$ \\
\hline $\begin{array}{l}\text { paesaggio caratterizzato dalla } \\
\text { presenza di grandi cascinali e da una } \\
\text { ricca rete di acque per l'irrigazione } \\
(\text { caratteristico, ad esempio, nel nord } \\
\text { ltalia }^{26} \text { ) }\end{array}$ & $\begin{array}{l}\text { le nuove unità } \\
\text { (originariamente abitate ciascuna da } \\
\text { circa una decina di famiglie) passano } \\
\text { da un'attività agricola orientata } \\
\text { soprattutto alla coltivazione di cereali } \\
\text { ad un'altra che assegna uno spazio } \\
\text { sempre più ampio alla produzione di } \\
\text { foraggi e e all'organizzazione } \\
\text { dell'allevamento }\end{array}$ \\
\hline $\begin{array}{l}\text { paesaggio con molte aree inutilizzate } \\
\text { (incolti, aree abbandonate, ...) }\end{array}$ & $\begin{array}{l}\text { abbandono delle aziende e dell'attività } \\
\text { agricola a causa della: } \\
\text { - marginalità e/o diseconomia del } \\
\text { sistema produttivo (improduttività) } \\
\text { - vicinanza all'insediamento urbano } \\
\text { che fa sì che le aree agricole siano } \\
\text { sottoposte ad una forte pressione } \\
\text { economica per la speculazione } \\
\text { edilizia }\end{array}$ \\
\hline
\end{tabular}

Come si può notare alcune delle situazioni sopra descritte sono il risultato diretto di cambiamenti che denotano positività e vitalità del sistema produttivo, mentre altre sono prodotte da modificazioni tendenti verso l'urbanizzazione, oppure verso una produzione di diverso tipo e di

$\overline{26}$ Cfr, L. BoRtolottI, Storia, città e territorio, Franco Angeli, Milano 1993, pp.226-227. 
minor qualità, o ancora verso una rinaturalizzazione di aree marginali o di aree trattate come tali. 
I paesaggi agresti futuri saranno ordinati, puliti e densi di macchine e di interconnessioni. (...)

Molte di queste interconnessioni, intubate, saranno visibili solo a occhi esperti. ${ }^{1}$

\section{PRINCIPALI CAMBIAMENTI DEL PAESAGGIO AGRARIO: ALCUNE FRA LE CAUSE PRINCIPALI DELLA PERDITA DI FIGURABILITÀ DEL PAESAGGIO AGRARIO}

Un'indagine come quella appena compiuta - ovvero l'individuazione delle principali componenti del paesaggio agrario, delle loro relazioni, del ruolo che ciascuna di esse riveste nella determinazione del paesaggio stesso e degli indicatori attraverso $i$ quali è possibile descrivere il suo stato di salute - tende ad essere inadeguata per spiegare e comprendere le forme del paesaggio vero e proprio se non viene accompagnata da una sorta di analisi dei principali processi storici che quelle forme hanno contribuito a costruire.

La metodologia classificatoria ha una sua validità solo se $i$ «fatti oggettivi» vengono rapportati alla dialettica storica che ne indica il loro divenire ${ }^{2}$.

L'agricoltura ha contribuito in modo determinante a dar forma al territorio e a creare l'aspetto di larga parte del paesaggio, fondamentalmente perché l'occupazione agricola del suolo, tra

1 R. VACCA, II paesaggio che ci sarà, in "ll paesaggio italiano: idee, contributi, immagini», TCl, Milano 2001, p.236.

2 M. MAROcco, II progetto dello spazio rurale, in «Rassegna di architettura e urbanistica» nn. 47-48, ago.-dic. 1980, Kappa, Roma, p.77. 
tutte le forme di occupazione dello spazio terrestre, può essere considerata quella storicamente e quantitativamente preminente: storicamente perché è la forma di occupazione che più a lungo, insieme all'insediamento di tipo abitativo, si è protratta nel tempo con variazioni poco apprezzabili; quantitativamente perché lo spazio richiesto dall'attività agricola è stato ed è ancora decisamente superiore a quello di qualsiasi altra attività dell'uomo.

L'analisi storica è importante in quanto strumento attraverso il quale individuare e comprendere $i$ processi e $i$ modi secondo $i$ quali avvengono le trasformazioni ed intendere il territorio secondo l'interpretazione che ne ha dato, in un dato contesto, i gruppi sociali che vi hanno instaurato un rapporto.

$\mathrm{Nel}$ passato, almeno fino alla prima guerra mondiale, l'evoluzione di questa forma di attività è però stata lenta e, di conseguenza, le trasformazioni sono state poco percettibili; dal periodo immediatamente successivo, e con progressive accelerazioni, il paesaggio agrario ha subito poi profonde mutazioni.

Le principali trasformazioni analizzate in questo capitolo sono quelle avvenute nel periodo più recente (dalla fine della seconda guerra mondiale ad oggi): sono state causate da fenomeni particolarmente significativi sia dal punto di vista territoriale $e$ paesistico sia dal punto di vista dell'interesse politico, sociale ed economico che hanno rivestito

- l'innovazione tecnologica,

- la riforma agraria,

- il processo di "urbanizzazione" e di "rururbanizzazione",

- la Politica Agricola Comune. 


\section{L'INNOVAZIONE TECNOLOGICA}

Dall'inizio del XX secolo l'introduzione della macchina ha prodotto una prima mutazione profonda del paesaggio agrario, che sino a quel momento era apparso come

una serie di quadri diversi tra di loro, ma sostanzialmente immutati nel tempo ${ }^{3}$.

La meccanizzazione ha permesso di compiere un notevole salto di qualità, ed è stata accompagnata da altre nuove tecnologie attraverso le quali è stato possibile migliorare la qualità della vita del contadino e la resa del suo lavoro: l'invenzione del trattore e dell'aratro "a versoio", che ha permesso lavorazioni del terreno migliori e più profonde, le nuove e sempre più avanzate conoscenze nel campo della nutrizione vegetale, per i concimi, e della chimica applicata alla protezione delle piante e delle genetica, per la scoperta di nuove varietà più produttive e/o più resistenti.

L'effetto di questi fattori tecnologici è stato dirompente perché in brevissimo tempo - rispetto ai tempi che il paesaggio agrario aveva sempre avuto nelle sue mutazioni anche profonde - i territori agricoli sono stati radicalmente trasformati a seguito di una spinta alla razionalità dell'uso del suolo, diventato sempre più specializzato e meno frazionato e discontinuo.

La causa principale è probabilmente da attribuirsi al fatto che in passato ciò che si cercava di ottenere era la massima produttività della terra, mentre oggi si mira a raggiungere la massima produttività del lavoro ${ }^{4}$. In questo modo quando si dice «un terreno molto produttivo» si intende in realtà

3 P. FABBRI, Natura e cultura del paesaggio agrario: indirizzi per la tutela e la progettazione, Città Studi, Milano 1997, p.9.

4 Per «massima produttività della terra» si intende il massimo di produzione per unità di superficie a parità di ore di lavoro; invece la «massima produttività del lavoro» indica il massimo di produzione per ora di lavoro a parità di superficie coltivata. 
un terreno sul quale il lavoro è più produttivo che su altri terreni ${ }^{5}$.

L'aumento della produttività del lavoro in agricoltura si è ottenuto anche attraverso quella che si potrebbe definire una «semplificazione dell'ambiente»: si sono, infatti, rimosse le siepi e le alberature, si sono colmati i fossi e i ruscelli, si sono abbattute le piccole opere di contenimento, e in molte zone la superficie agraria è stata "spiantata" ottenendo piani orizzontali o inclinati.

I processi così descritti hanno però attualmente ricadute negative sia sotto il profilo ecologico, sia sotto quello più propriamente paesistico.

Per il primo profilo la modernizzazione delle forme di conduzione e dei processi produttivi tradizionali ha portato ad una crisi ecologica, tendendo, in un certo senso, ad un capovolgimento di valori. Infatti, se il frazionamento dei terreni agricoli, con le relative forme tradizionali di conduzione, aveva costituito in qualche modo il fondamento della sostenibilità ecologica del paesaggio agrario italiano, il risultato più eclatante del processo di modernizzazione del settore primario ancorato soprattutto ad esigenze di mercato - è ora quello della progressiva scomparsa di molte specie animali e vegetali.

Sino alla rivoluzione industriale, infatti, l'organizzazione della società precapitalistica si era in larga parte fondata su forme di agricoltura che controllavano l'ecosistema naturale, inteso come base fondamentale dell'ambiente, caratterizzato dal reciproco adattamento di parti diverse, ciascuna con diversi modi di produzione. La storia offre un buon numero di esempi di regolamentazione cosciente dell'ambiente, anche se questo non deve autorizzare a pensare che esistesse una

coscienza ecologica nelle società preindustriali, anche perché la cultura occidentale ha di fatto posto l'uomo sempre al centro dell'universo, controllore dei fenomeni naturali ${ }^{6}$.

5 L. Contı, Paesaggio agrario: storia consumata e storia possibile, in Urbanistica Informazioni nn.112-113, INU edizioni, Roma 1991. 
Tale mentalità è stata talmente forte, e con radici talmente profonde, da permeare qualsiasi ambito: nel campo della pianificazione, ad esempio, ha contribuito a considerare il territorio come una specie di carta bianca, uno spazio nel quale potesse essere attuata qualsiasi destinazione d'uso. Nonostante questo, è possibile arrivare a dire che sino alla rivoluzione industriale la produzione agricola è avvenuta secondo ritmi che permettevano sia il reintegro delle energie e delle risorse attinte, sia l'assorbimento - naturale - degli scarsissimi inquinamenti prodotti. C'era in sostanza una sorta di «ordine ecologico».

A seguito dello sviluppo tecnologico postindustriale questo tipo di ordine è completamente saltato ed è stato sostituito da quello che potrebbe essere chiamato «ordine tecnologico». I livelli crescenti di rendimento e di produttività hanno fatto via via passare in secondo piano la questione ecologica globale, secondo il presupposto errato di un ambiente capace di auto-rigenerarsi e auto-purificarsi.

Soltanto nell'ultimo decennio è stata presa coscienza del fatto che si è di fronte ad una sorta di forbice, ossia che ad un aumento crescente della produttività (che comporta anche problemi legati al reperimento di nuovi mercati e, quindi, allo smaltimento delle derrate prodotte), corrisponde sempre un altrettanto massiccio impiego di tecnologie, con una conseguente concentrazione di inquinamenti (il cui smaltimento richiede a sua volta nuove tecnologie e così via, in un processo senza fine).

Contemporaneamente, da un punto di vista paesistico, con i processi di industrializzazione, di urbanizzazione e di esodo dalle campagne - dei quali si parlerà nell'ultima parte di questo capitolo nonché a seguito dei vantaggi economici prodotti dalla specializzazione produttiva che hanno portato a sfruttare superfici piuttosto vaste per la monocoltura facendo così scomparire il disegno risultante dall'alternarsi dei colori dovuto all'alternarsi di colture diverse, il paesaggio è andato

6 P. FABBRI, op. cit., p.20. 
sempre più trasformandosi esclusivamente in senso funzionale e utilitaristico, a scapito degli aspetti estetici.

II paesaggio rurale che l'agricoltura industrializzata offre al viaggiatore moderno è ormai quello dell'insipienza generalizzata ${ }^{7}$;

la perdita di una precisa riconoscibilità del territorio può essere considerata l'aspetto paesaggistico più negativo di questa nuova situazione.

G. CONTI, Abitare la distanza: dal radicamento rurale al teleantropismo metropolitano, in Urbanistica Informazioni Dossier n.9, INU edizioni, Roma 1997, pp.1-5. 


\section{LA RIFORMA AGRARIA}

Ricorda Emilio SERENI ${ }^{8}$ come l'evoluzione delle forme del paesaggio agrario sia stata condizionata non solo dalle innovazioni tecniche, ma anche e soprattutto dalle modificazioni dei rapporti di produzione dominanti delle campagne $\mathrm{e}$, più specificatamente, da certi risultati delle lotte $^{9}$ che le masse dei lavoratori e dei piccoli produttori agricoli hanno combattuto sia per un riscatto sociale, sia per un progresso vero e proprio dell'agricoltura.

Tra i più visibili effetti che queste lotte hanno avuto c'è quello della cosiddetta "riforma agraria" che nel $1950^{10}$ ha fatto sì che venissero eliminate vaste aree ad economia latifondista, trasformando profondamente il paesaggio agrario a causa della liquidazione, nella maggior parte dell'Italia, del sistema delle colture tradizionali a campi ed erba e del maggese nudo. La superficie agraria e forestale complessiva interessata dai provvedimenti era di 8.441 .658 ettari (ovvero il 29,33\% della superficie totale nazionale) ed era così ripartita: il 21\% nelle regioni agrarie di montagna, il $58 \%$ in quelle di collina e il $21 \%$ in quelle di pianura $^{11}$.

Nel corso degli anni '50, dunque, sulle terre del latifondo assegnate ai contadini, l'agricoltura di tipo estensivo è stata sostituita da una a carattere intensivo, che mirava ad una maggiore produttività.

8 E. SERENI, Storia del paesaggio agrario italiano, Laterza, Roma-Bari 1961; nuova ed. 1999.

9 Sono le lotte condotte dal movimento per la messa a coltura delle terre incolte, sviluppatosi poi nel movimento più grande per la conquista della terra, che ha fatto sì che dopo la seconda guerra mondiale "la proprietà latifondista vedesse l'estensione del suo dominio ridotta di circa 762.000 ettari” (Cfr. E.SERENI, op. cit., p.442).

10 I provvedimenti presi nel 1950 dal governo nazionale e definiti da questo "riforma agraria" furono nella sostanza: la Legge Sila del 12 maggio n²30 «per la colonizzazione dell'altipiano silano e dei territori jonici contermini» e la Legge stralcio del 21 ottobre n841, nonché l'istituzione di nove enti di riforma, ciascuno dei quali con un proprio ambito di operatività e con il compito di controllo del processo di trasformazione delle campagne.

11 I dati sono tratti da C. FALASCA, Territori agricoli e pianificazione urbanistica, NIS, Roma 1983. 
Nei terreni in cui furono realizzate infrastrutture, opere irrigue e case coloniche, ossia laddove gli assegnatari delle terre furono in grado di dare vita ad imprese capaci di elaborare e realizzare un piano di riforma, la trasformazione fondiaria riuscì a sorreggere una nuova rete di imprese contadine e i lineamenti del paesaggio agrario vennero definendosi con maggiore attenzione. Questa fu, però, la sorte solo di alcune zone particolari, già bonificate, collegate a strade e ferrovie, provviste rapidamente di un sufficiente parco animali ed attrezzi. Nella maggior parte dei fondi espropriati, invece, perché gravati da un eccessivo carico demografico, perché afflitti da una prevalenza di terreni collinari poveri ed argillosi, l'“informe desolazione" del latifondo venne via via sostituita da una frammentazione, il più delle volte casuale, in una miriade di piccole proprietà, dando così origine a scelte colturali scoordinate, incapaci di comporsi in un progetto comune, e ad un disegno del paesaggio agrario particolarmente disordinato.

È importante sottolineare come attraverso questo blocco di leggi di riforma fondiaria alcuni risultati siano stati certamente raggiunti (sono stati praticamente cancellati vecchi privilegi, servitù semifeudali, oneri parassitari), ma è altrettanto importante dire come i vizi originari della riforma, principalmente perché concepita fuori dalle istanze proprie del mondo contadino, attenuarono sensibilmente la portata innovatrice che avrebbero potuto avere i provvedimenti adottati.

Scrive M. Rossı DoRIA parlando della riforma agraria nella collana «Storia d'Italia» dell'Einaudi:

La riforma è stata concepita e attuata con un intervento rigidamente dall'alto, dal Ministero dell'Agricoltura e dagli enti di riforma, e come tale non ha potuto sottrarsi a tre inconvenienti il cui peso si è fatto sempre più sentire nel corso della sua realizzazione. Anzitutto, una pesante unità di direttive che mal si adattava alla grande varietà di situazioni che la riforma doveva affrontare; in secondo luogo, una totale assenza di democrazia interna che ha ridotto all'estremo la partecipazione attiva dei contadini, ad un piano del quale avrebbero dovuto essere i protagonisti. Quanto al terzo, esso è la conseguenza 
degli altri due: il costo delle operazioni è risultato molto più elevato di quello che sarebbe stato se fosse stato concepito e attuato con maggior elasticità ${ }^{12}$.

La considerazione principale è che alla base della riforma non vi fu un'impostazione di politica economica impostata sul lungo periodo, bensì orientata al raggiungimento dell'obiettivo immediato di assicurare uno sbocco alla disoccupazione, che negli anni dell'immediato dopoguerra affliggeva gran parte del paese, attraverso l'applicazione di un'economia agraria di pura e semplice sussistenza.

In questo quadro si può tranquillamente affermare che

non si trattò tanto di una vera riforma agraria, ma piuttosto di una parziale riforma fondiaria e, da questo punto di vista, ebbe anche una certa efficacia nel senso che la grande proprietà terriera assenteista ne ricevette un duro colpo ${ }^{13}$.

\section{IL PROCESSO DI “URBANIZZAZIONE” E DI “RURURBANIZZAZIONE”}

Nello stesso periodo, sia a seguito dell'assenza totale di politiche tese a far rimanere la popolazione nelle campagne, sia per la mutazione profonda che subiva la base economica del paese che si stava in brevissimo tempo industrializzando, i movimenti di popolazione interni alla nazione diventano vertiginosi, incidendo in maniera radicale sul territorio e sulla sua immagine complessiva.

Le conseguenze più immediate degli spostamenti di popolazione avvengono per ciò che riguarda l'armatura urbana, incidendo sulla fisionomia geografica del paese con vistose trasformazioni. Non è il primo processo di "urbanizzazione" che avviene in Italia e la sua entità non è minimamente paragonabile a quanto si era verificato in altre fasi cruciali dello sviluppo economico del paese, come quelle corrispondenti

\footnotetext{
12 M. Rossı DoRIA, La riforma sei anni dopo, in «Storia d'Italia», Einaudi, Torino 1976.

13

C. FALASCA, op. cit., p.18.
} 
alla realizzazione della rete ferroviaria. In quel caso, però, si era verificato un rafforzamento dei centri medio e grandi a scapito di quelli minori senza indebolire la popolazione rurale, come invece è avvenuto nel secondo dopoguerra quando la crescita urbana si è verificata proprio a spese della campagna.

La crescita rapida e concentrata del settore industriale, infatti, mette in moto un meccanismo di trasferimento settoriale e territoriale di una quota particolarmente consistente di forza lavoro agricola. Parallelamente, sempre a seguito della nascita e dello sviluppo dell'industrializzazione, la conversione dell'uso dei terreni da rurale ad urbano procede a ritmi sempre più sostenuti, a causa della crescita della domanda di inurbamento della popolazione.

Se le cause predominanti che hanno prodotto questo fenomeno di "esodo rurale" sono di natura economica, non bisogna sottovalutare quelle di carattere socio-culturale. Dal punto di vista economico, gli elementi che maggiormente hanno incentivato l'esodo rurale sono da un lato l'offerta di impiego, che nelle città è maggiore e più articolata, dall'altro lato gli aspetti qualitativi del "lavorare in città", che viene generalmente giudicato di un livello superiore perché meglio remunerato, meno faticoso e in condizioni migliori. Vi sono poi i fattori socio-culturali, per i quali la città viene preferita alla campagna perché sinonimo di "luogo di libertà"14 nel quale sono possibili relazioni sociali diversificate, o perché luogo privilegiato delle infrastrutture commerciali, scolastiche e sociali, o ancora perché luogo nel quale possono essere esercitate parecchie professioni che in campagna non avrebbero senso e/o mercato.

Inoltre, a supporto dell'urbanizzazione, nonché del bisogno crescente di far spostare persone e merci, si pone lo sviluppo dei trasporti che diviene rapidamente di massa e che, richiedendo reti di

14 J.B. ChARRIER, Geografia dei rapporti città - campagna, Franco Angeli, Milano 1994, p.47 
collegamento ferroviarie e stradali sempre più allargate sul territorio nazionale, contribuisce non poco ad un'occupazione del suolo diversa da quella propriamente agricola.

In sostanza, mentre le campagne si andavano spopolando all'interno di un rapporto sempre più squilibrato, le città raddoppiavano la loro consistenza.

Il dualismo città - campagna, una volta netto e preciso, si è via via sviluppato in modo più articolato, perché una realtà territoriale tradizionalmente indicativa di un mondo rurale - ossia definita dall'importanza delle attività agricole, dalla rarità dei servizi collettivi e terziari e da comunità amministrative formate da membri poco numerosi - oggi può mostrare situazioni del tutto differenti e contrastanti.

La stessa configurazione può, infatti, essere letta come indice di abbandono (interpretazione che ben si addice a diverse aree del Sud Italia e di montagna), o come indice di ricchezza (se ci si riferisce ad alcuni territori di pianura ad agricoltura avanzata). In più, i concetti relativi allo spazio rurale ed al suo paesaggio si vanno modificando, perché in molte zone l'urbanesimo penetra nella campagna, mentre molti elementi rurali restano inglobati nel paesaggio metropolitano a causa del ben noto modello di sviluppo a macchia d'olio della città.

Dal punto di vista morfologico, lo spazio territoriale, fino ad un determinato periodo (ossia fino al boom economico degli anni '60) era articolabile in tre fasce ben distinte:

- spazio urbano (edificato e sostanzialmente artificiale, caratterizzato dalla sovrapposizione e dall'intrecciarsi di funzioni);

- $\quad$ spazio rurale (spazio antropizzato e modificato dall'uomo in funzione dell'attività agricola);

- spazio naturale (con un'episodica presenza umana, non modificato volontariamente dall'azione dell'uomo se non in maniera marginale). 


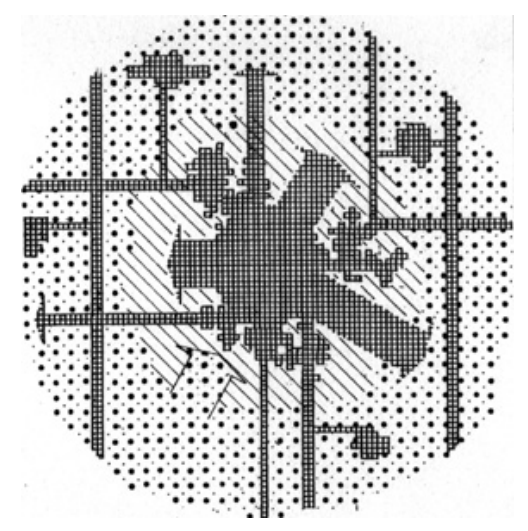

Figura 8. Situazione attuale
Questa suddivisione non è oggi più riconoscibile, in quanto sono aumentate le aree di frangia in cui i caratteri propri dello spazio urbano si mescolano con quelli dello spazio agrario e naturale.

In realtà appare sempre più difficile, soprattutto intorno alle grandi concentrazioni metropolitane, poter dire: qui è città, qui è campagna ${ }^{15}$.

Soprattutto nel corso degli anni '70, infatti, si è assistito all'affermazione di un diverso modello di organizzazione spaziale dei nuovi insediamenti - soprattutto quelli residenziali - per quanto riguarda le grandi città italiane ed europee: lo sviluppo dell'urbanizzazione ha assunto un carattere diffusivo, discontinuo dal punto di vista della contiguità del costruito, a bassa densità, pur restando in un certo senso strettamente legato ad alcune macro aree.

Sono stati cercati diversi termini per spiegare ed etichettare questo fenomeno, ma

forse il miglior modo di interpretarlo racchiudendolo in una formula stringata è quello di parlare di «metropolizzazione» ${ }^{16}$.

Verso la fine degli stessi anni '70, poi, venne introdotto il termine di «periurbanizzazione» con il quale si entrava nello specifico del fenomeno dell'urbanizzazione delle campagne, facendo riferimento a quei territori contigui alla città in cui $\mathrm{i}$ processi di urbanizzazione si confrontavano con attività agricole ancora pienamente funzionanti e con una società rurale ancora viva.

15 P. FABBri, op. cit., p.14.

16 R. Camagni, Processi di utilizzazione e difesa dei suoli nelle fasce periurbane: dal conflitto alla cooperazione fra città e campagna, in F. BosCACCI, R. CAMAGNI (a cura di) «Tra città e campagna: periurbanizzazione e politiche territoriali», II Mulino, Bologna 1994, p.25. 
I diversi modi di questo sviluppo periurbano in una porzione di territorio hanno sostanzialmente risposto ad alcune logiche localizzative, che potrebbero essere così riassunte:

- una logica di espansione quantitativa dello spazio urbanizzato per effetto della contiguità in direzione degli spazi verdi circostanti: si tratta - come già accennato - di una espansione a macchia d'olio, o meglio di un'espansione che avviene principalmente lungo le maglie delle reti infrastrutturali e che, successivamente, "vive" la saldatura degli spazi infra-reticolari;

- una logica di espansione di alcuni poli urbani (borghi preesistenti o nuove città create nel nulla) sia di carattere produttivo, che di carattere più residenziale, localizzati nell'hinterland rurale;

- una logica di localizzazione nello stesso hinterland di alcune grandi funzioni urbane;

- una logica, infine, di espansione secondo modelli insediativi qualitativamente nuovi e diversi di funzioni abitative caratterizzate da una bassa densità e da una forte interazione con aree verdi.

Sono - come è ovvio - logiche coesistenti, ma del tutto diverse sia per ciò che riguarda i processi decisionali che le hanno determinate, sia per gli effetti territoriali che esse hanno avuto in termini di consumo di suolo, domanda di mobilità, offerta potenziale di infrastrutture di trasporto pubblico e qualità dell'effetto urbano.

Altre trasformazioni, altrettanto profonde e negative, sono state provocate dai fenomeni di "rururbanizzazione"17, cioè dalla nascita di insediamenti residenziali su spazio agricolo legati funzionalmente a centri urbani di diversa importanza per prime e seconde case, e dalla avanzata caotica della stessa città verso la campagna.

17 II termine «rururbanizzazione» è stato coniato negli anni '60, intendendo "l'insediamento in comuni rurali di gente venuta dalla città, dove spesso continua a lavorare e che diventa, quindi, di fatto, pendolare" (in J.B. CHARRIER, op. cit., citando G. BAUER e J.M. ROUX, La rururbanisation ou la ville éparpillée, La Seuil, 1976). 
La differenza sostanziale rispetto alla "periferia tradizionale" e ai fenomeni di urbanizzazione è che non esiste più contiguità con il centro urbano, ma, al contrario, le nuove costruzioni sono disseminate in maniera particolarmente rada in un raggio anche di alcune decine di chilometri dall'agglomerazione centrale, sorpassando nella maggior parte dei casi i confini stessi dell'area urbana e di quella metropolitana. Alla base di questo fenomeno di "esodo urbano" è la ricerca di ambienti nei quali gli elementi del paesaggio rurale tradizionale sono ancora numerosi (in alcune occasioni addirittura dominanti), nonostante a ciò si associ una sempre più scarsa presenza di attività agricole.

Le cause che hanno prodotto questo nuovo modo di impegnare il territorio sono sicuramente molteplici, ma, anche se in maniera sommaria, è opportuno sviluppare quelle che potrebbero essere ritenute le più importanti $\mathrm{o}$, meglio, le cause primarie ${ }^{18}$.

La rururbanizzazione è, innanzitutto, frutto dell'insoddisfazione del cittadino nei confronti della vita urbana tradizionale e, in questa, delle condizioni di alloggio. Vivere in città di medie e grandi dimensioni è spesso diventato "sinonimo di affitto, di condominio"19, quando, al contrario, la popolazione preferirebbe vivere in una casa indipendente. Inoltre la qualità della vita nell'ambiente urbano si è andata sempre più deteriorando a seguito della crescita dell'inquinamento acustico ed atmosferico, della difficoltà di circolazione e di parcheggio delle macchine, dalla mancanza di comfort di molti alloggi popolari.

Un altro motivo importante che ha incentivato la "fuga dalla città" è stata l'attrazione esercitata dalle campagne suburbane, sia perché considerate ambiente gradevole, sereno immerso nel verde, sia perché

\footnotetext{
18 A questo proposito è utile vedere il cap.3 (pp.71-89) di J.B. CHARRIER, op. cit., "Le migrazioni città-campagna".

19 J.B. CHARRIER, op. cit., p.77.
} 
più economiche per ciò che riguarda l'acquisto ${ }^{20}$ di un terreno abbastanza vasto sul quale costruire un'abitazione di tipo monofamiliare.

Infine bisogna sottolineare come sia stato fondamentale, affinché avvenisse questo fenomeno della rururbanizzazione, il ruolo esercitato da un lato dai mezzi di trasporto e dall'altro dall'arrivo anche in campagna dei servizi che per lungo tempo erano appannaggio esclusivo della città (acqua corrente, gas, elettricità, telefono, ecc...). Soprattutto i primi, infatti, hanno permesso una diffusione capillare dell'insediamento rurale, tanto che, in molti casi, il tempo richiesto dagli spostamenti pendolari dalla campagna è inferiore a quello necessario al residente in periferia che deve attraversare da parte a parte una grande agglomerazione urbana.

Lo spazio rururbano viene generalmente definito in funzione di una serie di elementi.

Dal punto di vista formale, ad esempio, non è sempre detto che la rururbanizzazione si sviluppi in forma radiocentrica, bensì è anche possibile che presenti dissimetrie che il più delle volte dipendono dalla facilità o difficoltà di accesso e che, proprio per questo, avvengono in corrispondenza delle strade principali e delle vallate, assumendo la forma di filamenti lineari.

Dal punto di vista morfologico, l'insediamento rururbano può essere costituito da case di campagna restaurate e riadattate, anche se nella maggior parte delle occasioni si tratta di nuove abitazioni, differenti sia dalle precedenti, sia dalle villette di periferia, irregolarmente distribuite nel territorio e caratterizzate dal fatto che dispongono di molto terreno da destinare al giardino e all'orto.

Il fenomeno della rururbanizzazione si può quindi ritenere, più che la rivincita delle campagne, la riproduzione del fatto urbano perché, anche

20 È comunque importante far presente che alle economie realizzate sul costo dell'alloggio fanno riscontro maggiori costi delle spese di trasporto. 
in questo caso così come abbiamo visto accadere nel processo di urbanizzazione, tutto appare

di stampo urbano, ivi compresi i modi di vita e di consumo ${ }^{21}$.

I maggiori problemi legati tanto al fenomeno dell'urbanizzazione quanto a quello della rururbanizzazione riguardano principalmente:

- l'eccessivo consumo di suolo e di spazi un tempo dedicati prevalentemente all'agricoltura;

- il degrado ambientale che queste stesse aree hanno progressivamente vissuto sia a causa dell'inquinamento atmosferico, idrico, acustico, sia per la perdita e/o la modifica della loro immagine paesistica tradizionale.

\section{La Politica Agricola Comune}

L'agricoltura ha da sempre rappresentato uno degli obiettivi prioritari delle politiche decisionali della Comunità Europea prima e dell'Unione Europea adesso, non solo in ragione del suo peso a livello del bilancio comunitario, del quale assorbe circa il $50 \%$ dei fondi, ma soprattutto per la parte consistente di popolazione che si dedica all'agricoltura e per l'estensione del territorio destinato a questa attività.

La Politica Agricola Comune (PAC) consiste in una serie di norme e meccanismi che regolano la produzione, gli scambi e la lavorazione dei prodotti agricoli nell'ambito dell'Unione Europea, incentrando l'interesse maggiore in particolare sullo sviluppo rurale.

Le finalità della PAC sono in estrema sintesi le seguenti:

- incrementare la produttività dell'agricoltura, sviluppandone il progresso tecnico e assicurando uno sviluppo razionale delle produzioni;

21 J.B. ChARRIER, op. cit., p.89. 
- assicurare un tenore di vita equo alla popolazione agricola, migliorando il reddito individuale di coloro che operano nell'agricoltura;

- stabilizzare i mercati e assicurare prezzi ragionevoli dei diversi prodotti.

Le risorse finanziarie della PAC sono messe a disposizione dal cosiddetto Fondo Europeo Agricolo d'Orientamento e di Garanzia (FEAOG), che rappresenta una parte sostanziale del bilancio comunitario.

Questo fondo è suddiviso in due sezioni principali, la sezione orientamento, che contribuisce alle riforme agricole strutturali e allo sviluppo delle zone rurali (ad esempio gli investimenti per nuove attrezzature e tecnologie), e la sezione garanzia, che finanzia le spese inerenti l'organizzazione comune dei mercati (per esempio la promozione delle esportazioni).

La prima sezione ("orientamento") fa parte dei fondi strutturali che sono volti a promuovere lo sviluppo regionale e a ridurre le disparità in Europa; la seconda sezione ("garanzia") è di gran lunga la più importante e rientra tra le spese obbligatorie nel quadro generale del bilancio comunitario.

La PAC, durante i quattro decenni della sua esistenza, ha subìto numerose riforme dovute sia al fatto che è stato necessario tenere conto delle varie esigenze e richieste del mondo rurale, sia alla necessità di ricalibrare di volta in volta le strategie a seguito di alcuni effetti secondari indesiderabili che queste avevano prodotto.

II primo tentativo di riforma risale al 1968, quando il cosiddetto "Piano Mansholt" prevedeva la riduzione della popolazione attiva nel settore dell'agricoltura e proponeva, quindi, l'incoraggiamento alla formazione di unità di produzione agricola più grandi e più efficienti.

Durante gli anni '70 e i primi anni '80 le diverse riforme che subì la PAC furono tutte orientate a modernizzare l'agricoltura europea e a 
eliminare, o a ridurre, $\mathrm{i}$ problemi nati dallo squilibrio persistente tra l'offerta e la domanda di prodotti agricoli, con la conseguente crescita delle eccedenze. Nel 1985 fu pubblicato un libro verde sulle "Prospettive della politica agraria comune" con il quale si dava seguito ad una proposta di riforma avanzata dalla Commissione Agricoltura nel 1983 e si cercava di ristabilire un giusto equilibrio tra offerta e domanda, di formulare nuove soluzioni per ridurre la produzione dei settori che si trovavano in difficoltà e di proporre possibili alternative per il futuro della PAC stessa.

La politica attuata fino ad allora, infatti, rispondeva principalmente all'esigenza dei primi anni '50 di assicurare un crescente flusso di prodotti agricoli ad una popolazione in aumento, i cui fabbisogni alimentari apparivano destinati ad incrementarsi parallelamente al miglioramento dei redditi.

Nel maggio del 1992, cessate queste condizioni, c'è stata una nuova sostanziale riforma che consisteva essenzialmente nella riduzione dei prezzi agricoli al fine di renderli più competitivi sul mercato interno e su quello mondiale, nell'assegnazione di importi compensativi per le perdite di reddito subite dagli agricoltori e in altre misure relative ai meccanismi di mercato e, soprattutto, alla protezione dell'ambiente.

È dall'inizio degli anni '90 che la tematica ambientale ha cominciato ad acquistare una crescente importanza all'interno del nuovo orientamento della PAC, allo scopo di salvaguardare, proteggere e migliorare la qualità dell'ambiente, contribuire alla protezione della salute umana e garantire un'accorta e razionale utilizzazione delle risorse naturali. Questa scelta di dotarsi di una politica ambientale comune consente all'Unione Europea di affrontare in maniera organica alcuni problemi che, per loro natura, hanno un'importanza sovranazionale e che richiedono quindi, per una loro corretta soluzione, un forte coordinamento degli interventi. 
Le linee di azione di questa nuova politica ambientale partono dalla constatazione del ruolo che l'agricoltura ha sempre svolto a favore dell'ambiente $^{22}$ e di come essa sia mutata, come abbiamo già visto, negli ultimi 40 anni, soprattutto a seguito dell'introduzione di tecnologie agricole più intensive e sotto la pressione di altre attività economiche.

I principali problemi individuati sono soprattutto il deterioramento di taluni habitat terrestri e l'estinzione di alcune specie (vegetali ed animali) a causa di disturbi ambientali dovuti anche all'attività agricola intensiva, così come per il prossimo futuro preoccupa il degrado dei suoli che è frutto non solo dell'uso intensivo delle risorse per l'agricoltura, ma dell'abbandono di molte parti di territorio prima dedicate ad attività del settore primario, della maggiore evaporazione di ammoniaca provocata dagli allevamenti intensivi, dell'acidificazione dei suoli e delle modifiche apportate al paesaggio.

Fra le strategie adottate ${ }^{23}$ (soprattutto incentivi di tipo economico) dalla PAC per cercare di scongiurare questi problemi sono da ricordare in estrema sintesi:

- l'introduzione di aiuti comunitari agli agricoltori che applicano pratiche compatibili con l'ambiente;

- un sistema di aiuti per la protezione o il miglioramento dell'ambiente nell'ambito dell'impresa agricola o dell'industria agro-alimentare;

- le limitazioni e i divieti alla concessione di aiuti agli allevamenti intensivi;

22 È utile a questo proposito riprendere le conclusioni del documento "Ambiente e Agricoltura" dell'agosto del 1988, nel quale si rifletteva proprio sul ruolo che l'agricoltura ha svolto e svolge a favore dell'ambiente:

... l'agricoltore dovrebbe diventare consapevole che il suo ruolo non è limitato alla produzione agricola stessa, ma si estende anche al mantenimento dell'ambiente rurale che egli gestisce. Ciò implica che la società deve accettare il fatto che l'agricoltore in quanto gestore dell'ambiente rende un servizio pubblico che merita un'adeguata remunerazione.

23 Le strategie elencate fanno riferimento soprattutto al Reg.797/85 relativo al miglioramento dell'efficienza delle strutture agricole, al Reg.1094/88 (con le successive integrazioni dei Regg.1272 e 1273/88) che ha introdotto il "set-aside" e al Reg.768/89 che istituisce un regime di aiuti transitori al reddito agricolo. 
- la concessione di indennità compensative per mantenere l'agricoltura nelle zone di montagna;

- le misure per la protezione delle foreste;

- le misure volte ad incoraggiare l'estensivazione della produzione e il ritiro delle terre dall'attività agricola ("set-aside").

Nel luglio del 1997 la Commissione ha proposto un nuovo adeguamento della PAC soprattutto in ragione dell'allargamento dell'Unione Europea ai paesi dell'Europa centrale e orientale e nell'ottica dei preparativi per l'introduzione della moneta unica: una tappa fondamentale di questo ulteriore cambiamento è stata Agenda 2000. Scopo prioritario della nuova riforma è approfondire ed estendere la riforma del 1992, sostituendo le misure di sostegno dei prezzi previste allora con aiuti diretti.

La riforma ha dunque previsto misure intese soprattutto a rafforzare la competitività delle materie prime agricole sia sul mercato interno, sia su quello mondiale, migliorando principalmente la qualità dei prodotti e la loro sicurezza; integrare maggiormente nella PAC questioni ambientali e strutturali; semplificare la legislazione in materia agraria e decentralizzarne l'applicazione, soprattutto con l'obiettivo di avere una maggiore chiarezza, trasparenza e accessibilità di norme e regolamenti.

In particolare le linee direttrici sulle quali si basa Agenda 200 sono:

- competitività, che deve essere assicurata con una diminuzione dei prezzi sufficiente a garantire l'aumento degli sbocchi interni e una partecipazione al mercato allargata al mercato mondiale (questa diminuzione dei prezzi dovrebbe essere compensata da un aumento degli aiuti diretti in modo tale da conservare il livello del reddito);

- ripartizione dei compiti fra Commissione UE e Stati membri, sia nel caso si tratti della compensazione sotto forma di aiuti diretti, sia per misure di sviluppo rurale nel quadro di una programmazione globale (questo sforzo verso una decentralizzazione dei poteri e delle risorse 
è necessario vada di pari passo con la semplificazione delle normative);

- politica di sviluppo rurale globale e coerente, ovvero una politica che sia volta ad integrarsi con le politiche di mercato e a garantire che la spesa agricola contribuisca meglio di quanto non è avvenuto in passato all'assetto territoriale e alla protezione della natura. 
Le esigenze dello sviluppo e quelle della salvaguardia sembrano a prima vista incompatibili, e così è se il processo della pianificazione e gestione del territorio segue la visione squisitamente antropocentrica come ha fatto finora. ${ }^{1}$

\section{LE RAGIONI DI UN'INVERSIONE DI TENDENZA: \\ IL PAESAGGIO AGRARIO DA AMBITO RESIDUALE AD \\ ELEMENTO DI CONNESSIONE DELLE EMERGENZE AMBIENTALI E PAESISTICHE E "PRESIDIO" PER IL TERRITORIO}

Se l'agricoltura è sempre stata l'uso più diffuso del territorio, le aree agricole, non avendo storicamente avuto specifica regolamentazione, sono state di fatto considerate come aree disponibili per qualunque tipo di trasformazione, proprio per la loro natura di "territori di mezzo", "aree bianche" rispetto ad una normativa - quella urbanistica - il cui scopo principale è stato quello di una gestione quantitativa del costruito ammissibile e di una ricerca di nuovi ambiti disponibili per l'edificazione.

Le politiche economiche finora dominant ${ }^{2}$, poi, hanno di fatto considerato l'agricoltura come un settore residuale, circoscritto ed orientato unicamente alla produzione per il mercato.

P. FABBri (a cura di), Paesaggio, pianificazione, sostenibilità, Alinea, Firenze 2003, p.9.

2 A questo proposito si veda quanto scritto nel capitolo 2. 
In tempi recenti, però, dopo essere state emarginate dallo sviluppo industriale e/o invase dalla crescita fisica degli insediamenti urbani, le aree agricole sono state finalmente "riscoperte" e "ri-considerate" come una risorsa preziosa per la protezione dell'ambiente e del territorio e per l'equilibrio economico, e come spazio abitabile e matrice storica di specifiche realtà locali.

In realtà l'agricoltura costituisce potenzialmente il centro di una larghissima matrice intersettoriale di attività, produzioni, servizi, opportunità. ${ }^{3}$

In buona sostanza, più che ad una inversione di tendenza, si sta assistendo ad un diverso approccio verso le aree agricole. $\dot{E}$ sempre più riconosciuto come la terra e le sue risorse primarie siano chiamate a svolgere un ruolo strategico nellintegrazione territoriale ed ambientale e nella stessa riqualificazione urbana.

Il paesaggio agrario comincia infatti ad assumere qualità $e$ compiti molto diversificati:

- ai territori dell'agricoltura industrializzata viene chiesto di garantire le funzioni propriamente produttive ed un equilibrio economico complessivo;

- alle aree agricole tradizionali viene attribuito il compito di proteggere e mantenere l'equilibrio biologico complessivo del territorio;

- tutto il paesaggio agrario, infine, viene riconosciuto come "produttore di cultura", ovvero come elemento attraverso cui individuare i "segni storici" e ritrovare l'identità dei luoghi.

3 ANCI Toscana, Agricoltura e territorio: un laboratorio per lo sviluppo sostenibile della Toscana, Centro A-Zeta, Firenze 1996, p.14. 
Un quadro siffatto delinea inoltre una figura del tutto nuova di agricoltore,

un operatore che deve sintetizzare diverse qualità: produttore, ma anche "giardiniere", gestore, tecnico, animatore; un agricoltore che deve assumersi l'onere di servire alla collettività e deve sapere dialogare con i poteri pubblici che le fanno da tramite. ${ }^{4}$

4 M. Di Rosa, La salvaguardia del paesaggio rurale. Problemi, obiettivi e strumenti per una gestione "sostenibile", in M.E. BALDI (a cura di) «La riqualificazione del paesaggio», La Zisa, Palermo 1999, p. 311. 


\section{L'EQUILIBRIO PRODUTTIVO ED ECONOMICO}

In primissimo luogo, si sta assistendo ad una essenziale riorganizzazione del ciclo produttivo agricolo, in una direzione che mira a ridurre gli input energetici e ad incrementare la diversità biologica e le coltivazioni autoctone.

Come è stato detto nel capitolo precedente, gli aiuti comunitari vanno proprio in questa direzione: sono destinati alla riduzione dei consumi di concimi e pesticidi, alla estensivizzazione delle produzioni vegetali, alla diffusione di metodi e processi naturali (con la reintroduzione di rotazioni agrarie e della concimazione verde) e all'introduzione di tecnologie appropriate e compatibili con l'ambiente, alla cura dei terreni agricoli e forestali abbandonati, alla formazione degli agricoltori, alla ri-naturalizzazione delle aree agricole e al miglioramento delle aree boschive esistenti.

Ciò a cui si sta cercando di arrivare, in definitiva, è la ricostruzione di agro-sistemi biologicamente complessi e diversificati mediante l'introduzione di tecniche di irrigazione più razionali e con l'incentivazione di metodi produttivi biologicamente controllati. Le azioni progettuali, così come la programmazione economica di larga e di piccola scala, sempre più mirano ad una riduzione dell'impiego delle tecnologie, senza che questo voglia dire tornare alla vita disagiata nei campi che si faceva fino all'inizio del secolo scorso ${ }^{5}$; spingono verso un mantenimento (o un ripristino) delle coltivazioni delle superfici agrarie, specialmente di quelle collinari e montane che più hanno subito una sorta di desertificazione o comunque un forte abbandono, verso le policulture e le maggiori articolazioni vegetazionali che sono andate progressivamente distrutte.

5 Cfr. L. CHILÒ, La rivoluzione delle biotecnologie e la trasformazione del paesaggio agrario, Grafo, Brescia 1992, pp.114-115. 
Tra i vari metodi di produzione agricola, il metodo biologico oltre la massima riduzione degli inputs chimici ed energetici che possono avere conseguenze nocive per l'ambiente, oltre che dare origine alla presenza di residui nei prodotti agricoli.

L'agricoltura biologica, infatti, sancisce il ruolo fondamentale del terreno nel metodo di produzione:

al posto dei fertilizzanti e degli antiparassitari di origine sintetica usati nell'agricoltura convenzionale vengono valorizzate le rotazioni, le fertilizzazioni mediante sovesci, l'uso di prodotti di origine naturale per la difesa delle produzioni. $^{6}$

Non si tratta di recuperare tout court il "vecchio" metodo di produzione agricola, bensì si cerca di rivisitarlo alla luce delle conoscenze più moderne, ponendosi come obiettivo principale quello di preservare e migliorare lo stato di fertilità del terreno.

Tra gli elementi che più possono arricchire la produzione agricola non può, dunque, non tenersi in conto, dal momento che essa è innanzitutto fornitrice di prodotti alimentari, la qualità stessa del prodotto.

Se la standardizzazione e la banalizzazione dei cibi è cresciuta insieme alle esigenze del consumatore e della distribuzione, è opportuno valutare la crescita delle esigenze legate proprio alla qualità del prodotto ed alle sue caratteristiche intrinseche di sanità. I casi avvenuti negli ultimi anni (dal pollo alla diossina al "fenomeno mucca pazza") sono testimoni del fatto che non vi è esclusivamente una mera ricerca "di lusso" di prodotti biologicamente compatibili, ma si riscontra soprattutto una sempre più crescente e diffusa consapevolezza dei rischi - ormai invasivi - della produzione industriale.

Ponendo comunque attenzione alle esigenze ed alle opportunità della distribuzione, è diventata prioritaria la possibilità di riconoscere

6 R. Russu (a cura di), L'agricoltura e le pressioni sull'ambiente, ARSIA e Regione Toscana, Firenze 1997, p.23. 
delle "specificità" ai cibi, ovvero incentivare la ricerca di una loro riconoscibilità, che deve essere strettamente legata al luogo e al modo della produzione. L'identificabilità, dunque, costituisce il necessario volano per ricreare un rapporto - sia pure virtuale - tra prodotto e luogo, tra specificità locale ed esigenze della distribuzione.

\section{LA PROTEZIONE DELL'AMBIENTE E IL RIEQUILIBRIO ECOLOGICO}

Appartiene agli ultimi anni la consapevolezza che buona parte del processo di riqualificazione dell'ambiente può essere attuato attraverso una regolamentazione delle attività agricole che abbia come obiettivi primari la rinaturalizzazione degli ambiti produttivi e il recupero della continuità degli spazi naturali al fine di ricomporre l'unitarietà di tutto il sistema paesistico.

Per avviare un processo di questo tipo è necessario e prioritario porre alla base della "nuova" agricoltura la considerazione del ruolo che essa può assumere nella conservazione della natura e nel miglioramento qualitativo delle condizioni dell'ambiente.

È sostanzialmente necessario riconoscere, incentivare e confermare il ruolo importante che l'agricoltura può svolgere nella produzione di beni ambientali e di risorse paesistiche. Una particolare attenzione viene data alle aree d'interfaccia tra la città e la campagna, nelle quali è più pressante la necessità di reagire ai processi di degrado in atto che riguardano tanto i manufatti, quanto le opere di regimazione delle acque e, più in generale, le infrastrutture proprie della campagna.

Le risorse utilizzate dall'agricoltura sono dunque rivolte a produrre contemporaneamente beni agricoli e servizi ambientali, assecondando in questo modo quei principi che la Comunità Europea ha fatto propri con la sua ultima riforma. In determinate situazioni di "margine produttivo", soprattutto, 
la produzione di autentici beni immateriali di natura territoriale (di tutela ambientale, di sviluppo sociale, ecc... $)^{7}$,

va affiancandosi alla mera produzione quantitativa di beni alimentari.

II rilancio delle aree rurali di tipo tradizionale (ovvero di tutte quelle aree agricole non ancora interessate dall'agricoltura di tipo industriale) può svolgere un ruolo strategico in questa direzione di «servizio ambientale», proprio perché quelle aree possiedono caratteristiche strutturali adatte

per sviluppare una forte ed equilibrata integrazione ambientale del territorio nel suo complesso ed in particolare per togliere dalle condizioni di insularità le aree ad alta naturalità diffusa ${ }^{8}$.

Ciò che deve avvenire è sostanzialmente una forte e capillare integrazione del paesaggio agrario, e di tutti i suoi elementi componenti, tanto con i cosiddetti paesaggi naturali, quanto con quelli completamente artificializzati.

Nell'equilibrio biologico e nello stato di salute complessivo del territorio, lo spazio rurale ha finalmente cominciato a svolgere il compito ad esso più congeniale di sistema di trasmissione fra i paesaggi naturali e quelli artificiali, mediante l'uso di connessioni lineari, utilizzando opportune specie vegetali che consentono la diffusione di diverse forme biologiche e superando sia la banalizzazione degli ecosistemi extraurbani, sia l'attuale frammentazione ecologica del territorio.

È ponendo l'interesse su questi ultimi temi che risulta determinante prestare attenzione alla progettazione dei corridoi ecologici, che sono in definitiva costituiti soprattutto dai sistemi lineari di siepi e di fasce

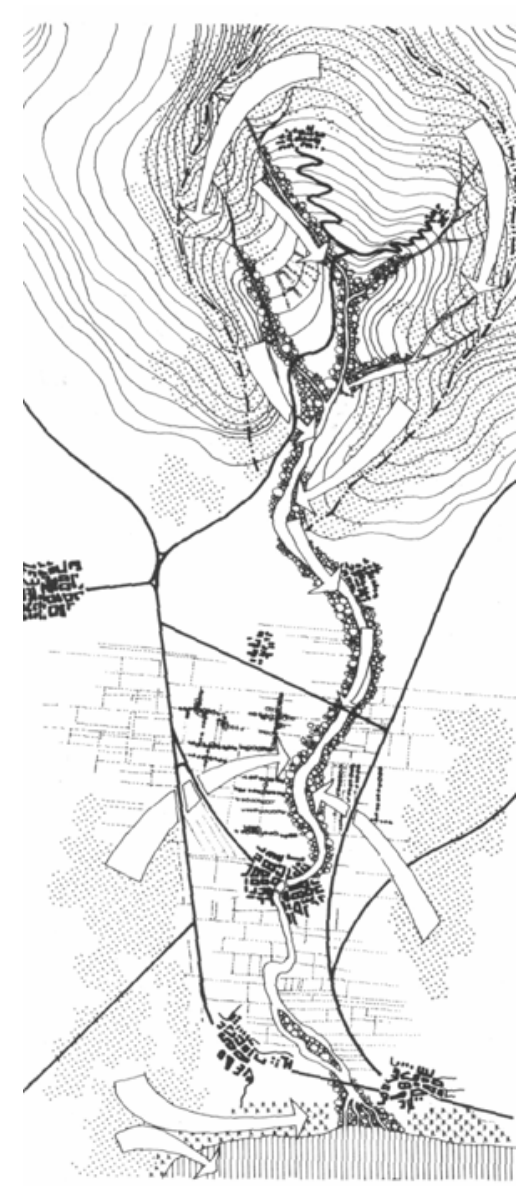

Figura 9. L'importanza strategica dei paesaggi agrari nei contesti seminaturali.

7 M. DI RosA, op. cit., p. 310.

8 A. Tosı, Assetti agricoli e vegetazionali sostenibili contro il degrado e la diffusione insediativa periurbana, in A. Tosı (a cura di) «Degrado ambientale periurbano e restauro naturalistico», Franco Angeli, Milano 1999, p.12. 
arboree ed arbustive posti nei territori agricoli e legati alle loro infrastrutture (corsi d'acqua, strade interpoderali, ecc...).

\section{IL RICONOSCIMENTO DEI “SEGNI STORICl” PER RITROVARE L'IDENTITÀ DEI LUOGHI}

La produttività sociale dell'agricoltura non si può misurare solo in termini di puro rendimento economico, ma in termini di benefici sociali, di economie esterne, di ricchezza sociale. La conservazione del paesaggio, infatti, sempre più viene considerata un arricchimento collettivo, strettamente collegato al patrimonio artistico e culturale.

L'agricoltura, come abbiamo già avuto modo di vedere, non viene più considerata esclusivamente dal punto di vista economico-produttivo tradizionale: sono state infatti fatte scelte politiche orientate ad indirizzare

i sistemi agricoli verso forme di organizzazione che assolvono anche complesse funzioni extra-agricole, sviluppando il ruolo sociale e pubblico della gestione del territorio agricolo. ${ }^{9}$

Nella Convenzione Europea viene ampiamente sottolineato il significato culturale intrinseco ad ogni paesaggio, più volte ripreso anche da Roberto Gambino che in uno dei suoi ultimi saggi ricorda che

II paesaggio ha sempre - anche quando i suoi connotati naturali sembrano esenti da ogni contaminazione antropica, ... - un imprescindibile significato culturale, legato ai contenuti ed alle modalità dell'esperienza paesistica, ai legami inscindibili tra ecosfera e semiosfera, alle motivazioni e ai condizionamenti degli stessi "sguardi" che vi si orientano. ${ }^{10}$

9 ANCl Toscana, op. cit., p.19.

10 R. Gambino, Maniere di intendere il paesaggio, in A. ClementI (a cura di), «Interpretazioni di paesaggio», Meltemi, Roma 2002, p.57. 
Intendendo, quindi, il paesaggio agrario come fondamentale produttore di "cultura", gli si possono riconoscere due valori molto importanti: uno legato alla memoria dei luoghi, uno legato a ciò che riguarda il ruolo ricreativo che esso assume, soprattutto nelle aree più prossime agli insediamenti urbani metropolitani.

In primo luogo, per ciò che riguarda il riconoscimento di un valore di memoria, si intende il paesaggio come un «libro di storia» ${ }^{11}$ del territorio e della società.

La lettura delle componenti fisiografiche originarie, che sono state spesso cancellate in modo irrecuperabile (corsi d'acqua interrati, fiumi arginati, boschi abbattuti, ecc...), diventa conditio sine qua non per riappropriarsi della «forma data» interamente o parzialmente obliterata. ${ }^{12}$

È necessario comprendere e progettare una determinata porzione di territorio, partendo dal riconoscimento dei caratteri componenti il paesaggio agrario e mettendo in evidenza le sequenze e la struttura della geologia storica, della pedologia, della fisiografia, dell'idrologia e della associazioni vegetali. Proprio dall'interpretazione di questi elementi derivano la conoscenza della storia di un luogo e la conservazione e la valorizzazione della sua identità intrinseca.

La seconda "attività culturale" alla quale è chiamato il paesaggio agrario oggi, importante tanto quella precedente, consiste nel valore sociale che esso assume: ovvero nell'insieme di tutte quelle funzioni di compensazione di ciò che la vita metropolitana non è in grado di offrire.

Il riferimento è al ruolo pedagogico-formativo e, connesso a questo, al ruolo ricreativo che le aree rurali periurbane oggi assumono.

L'attenzione privilegiata al periurbano è legittimata dalla vulnerabilità delle aree stesse, che sono state chiamate ad ospitare la maggior parte della crescita urbana. Sono però soprattutto queste zone che rivestono

\footnotetext{
11 Cfr. M. Dı Rosa, op. cit., pp.305-335.

12 Cfr. A. Tosı, op. cit., pp.7-16.
} 
l'importante ruolo di equilibrio complessivo per tutta la popolazione urbana, tanto che è necessario trovare per questa porzione di territorio nuove forme - progettuali e gestionali - per superare il conflitto tra urbano e rurale, tra "pieni" e "vuoti".

In questo quadro di suburbanizzazione agricola, la funzione urbanistica dell'agricoltura dovrebbe consistere nell'offerta di spazi verdi produttivi tra insediamenti urbani, industriali ed infrastrutturale; nell'offerta di occasioni di lavoro, eventualmente legato ad una attività principale, per alcuni proprietari coltivatori, in un disegno complessivo che veda un aumento della produzione lorda agricola. ${ }^{13}$

La ricerca di nuove funzioni per le aree perturbane che continuano ad essere caratterizzate da valenze agricole rappresenta dunque un campo di grande rilievo nell'affrontare sia il problema legato ai margini urbani, sia proprio quello della produzione e ricostruzione dei valori ambientali nello spazio urbano, che, di questi ultimi, è sempre meno provvisto.

13 M. MARocco, Politiche e strumenti di intervento nelle aree agricole peri-urbane, in «Agricoltura-Ambiente» n. 8, giu., Graf. Palombi, Roma 1980, p.31. 
II giudizio di valore è sempre soggettivo, soprattutto quando si ha la sovrapposizione di valori di diverso carattere: da quelli estetici a quelli biologici, da quelli fisici a quelli storici, da quelli etnici a quelli sociali. Le situazioni da valutare non sono poi solo le realtà esterne, visive, viste come in una fotografia, ma le strutture, i processi produttivi, i contenuti sociali. ${ }^{1}$

\section{GLI INDICATORI DEL PAESAGGIO AGRARIO}

L'osservazione e lo studio del paesaggio necessita sempre più di una valutazione tanto dei singoli elementi che 10 compongono e dei fenomeni che vi si svolgono, quanto del suo insieme e della sua struttura; ovvero è necessario interpretare criticamente il complesso sistema in cui il paesaggio stesso si articola.

Recentemente - dalla Carta di Napoli, così come dalla Convenzione Europea - si evince come, oltre ad individuare le diverse organizzazioni spaziali del paesaggio, sia fondamentale studiarne lo stato di salute, nonché l'evoluzione che nel tempo esso ha vissuto, attraverso l'individuazione e l'uso appropriato di specifici indicatori.

1 G. BARBIERI, Evoluzione del concetto e della funzione dei parchi nella politica del territorio e dell'ambiente, in G. BARBIERI, F. CANIGIANI, "Le ragioni dei parchi e l'Italia "protetta" ", quaderno n.15 dell'Istituto di Geografia dell'Università di Firenze, 1989, p.236. 
Tutto ciò è diventato un'esigenza sempre più imprescindibile, dal momento in cui si è posto il problema di seguire con maggiore capacità conoscitiva i processi territoriali in atto, di prevederli e, in qualche modo, di controllarne anticipatamente l'evoluzione mediante una programmazione territoriale ed economica.

L'interesse per il monitoraggio complessivo ed insieme analitico del territorio si è dunque velocemente accresciuto, sia tra gli studiosi, sia tra gli operatori territoriali (pubblici e privati che siano). È inoltre un dato di fatto come l'utilizzo degli indicatori a supporto delle politiche territoriali ed economico-funzionali sia ormai oggetto di elaborazioni e decisioni importanti, assunte in primo luogo da organismi internazionali ed europei (l'Agenda 21 ne costituisce certamente un esempio) e di numerosi approcci ed analisi, che si differenziano per metodologie e risultati anche allinterno di una stessa disciplina.

Per questo motivo, a mio avviso, è importante in questa ricerca, anche se in maniera sintetica e sommaria, studiare quali sono gli indicatori attraverso i quali è possibile descrivere lo stato di salute del paesaggio agrario e dei cambiamenti complessi che avvengono al suo interno, considerando però che

ad ogni realtà o scala appartengono indicatori propri che devono essere scelti in base alle caratteristiche proprie del paesaggio in esame preliminarmente individuate ${ }^{2}$.

In questo capitolo si cercherà di valutare sinteticamente le potenzialità ed i limiti dell'utilizzo di questi nuovi strumenti, anche

2 AA.Vv., Carta di Napoli. Il parere degli specialisti sulla riforma degli ordinamenti di tutela del paesaggio in Italia, Napoli ottobre 1999, p.3. 
attraverso la lettura di alcune fra le principali ricerche e metodologie recentemente elaborate sul tema, prendendo spunto dalle categorie proposte da due diverse esperienze:

- il Vo volume del "Manuale $A A A$ degli indicatori per la Valutazione di Impatto Ambientale" curato da Alessandro G. Colombo e Sergio Malcevschi nel 1999;

- gli Indicatori per l'integrazione della problematica ambientale nella Politica Agricola Comune proposti dalla Commissione UE nel 2000. 


\section{Considerazioni generali}

Generalmente l'indicatore si identifica come un elemento o un parametro che,

in relazione al caso in esame, stabilisce, attraverso il confronto del suo stato ottimale con le variazioni alle quali esso è sottoposto, il grado di compatibilità di una scelta di pianificazione con la situazione di partenza. ${ }^{3}$

L'indicatore si riferisce solitamente ad un parametro che, presentando una stretta relazione con un determinato fenomeno, è in grado di fornire informazioni sulle caratteristiche dell'evento esaminato nella sua globalità, nonostante ne rappresenti solo una parte.

Funzione principale dell'indicatore è la rappresentazione sintetica dei problemi, indagati in modo però da conservare il contenuto informativo dell'analisi. ${ }^{4}$

La tendenza che si può riscontrare nelle recenti metodologie di applicazione degli indicatori è quella che li classifica in base a considerazioni sulle cause e sugli effetti che un determinato evento genera in un preciso contesto, suddividendoli in:

- indicatori di pressione, utilizzati per descrivere le pressioni esercitate dalle diverse attività umane in atto nell'ambito oggetto dell'analisi;

- $\quad$ indicatori di stato, per misurare la quantità e qualità dei fenomeni fisici, biologici, chimici in atto e per fotografare e descrivere in un dato momento le risorse presenti in quell'ambito;

3 M. GIUDICE, Gli indicatori ambientali: strumenti per la pianificazione del territorio, in «Parchi» n.0, mag., Maggioli, Rimini 1990.

4 ArPav, Rapporto sugli indicatori ambientali del Veneto - anno 2000, pubblicato sul sito www.arpa.veneto.it/via/report.htm, 2000. 
- indicatori di risposta, riferiti alle misure prese dalla società per prevenire, compensare, migliorare, adattare i cambiamenti avvenuti nella porzione di territorio esaminata.

Nella scelta dei possibili indicatori è essenziale tener presente, in quanto strumenti di valutazione del livello qualitativo di una determinata situazione e/o porzione di territorio, che sotto alcuni punti di vista sono importanti, ma che, nello stesso tempo, presentano ancora dei limiti sotto altri.

II pregio maggiore che può esservi attribuito è il contributo sostanziale che hanno portato al tema della valutazione delle qualità ambientali e paesistiche. L'inadeguatezza degli strumenti a sostegno delle decisioni riscontrata sino ad oggi, infatti, ha sempre più sollecitato a ricercare e ad introdurre nuovi strumenti di controllo e sistemi di giudizio sugli effetti che derivano da progetti trasformativi, che siano largamente motivati e condivisi.

Gli ambiti di valutazione nei quali, solitamente, è stato articolato il quadro diagnostico, sono stati principalmente due:

- un ambito esterno, ovvero il contesto ambientale e territoriale entro il quale si opera e del quale si mira a conoscere la qualità dell'ambiente e/o del paesaggio e il suo stato, i fattori di pressione in atto o potenziali, le politiche attivate;

- $\quad$ un ambito interno al quale ci si riferisce per valutare le politiche e le modalità d'azione adottate dalle pubbliche amministrazioni e dagli altri soggetti che operano sul territorio.

Un'altra valenza di grande importanza da riconoscere allo "strumento indicatore" è, senza dubbio, la sua capacità di dare un'informazione e una rappresentazione sintetica delle caratteristiche della situazione territoriale che si sta indagando e dei problemi in atto, rendendo visibile un fenomeno e/o un processo che non sarebbe 
immediatamente percepibile e semplificando molto la comunicazione e la divulgazione dei risultati.

Nello stesso tempo, però, è fondamentale essere consapevoli del livello di incertezza che ancora permane su alcuni aspetti che riguardano gli indicatori, proprio per non correre il rischio di attribuire a questa tecnica ruoli che essa non può ricoprire.

È innanzitutto necessario tenere in dovuto conto il fatto che gli indicatori analizzano, qualificano e quantificano fenomeni e processi che non sono facilmente misurabili. Le realtà territoriali entro le quali ci si trova ad operare, infatti, sono il più delle volte complesse, costituite da fattori, fenomeni e processi che non sono direttamente rilevabili.

I numerosi indicatori e parametri che possono essere utilizzati, dunque, dovrebbero essere interpretati come spie significative dell'andamento di una certa realtà: andamento che viene studiato operando una sorta di bilancio. II ruolo che ad essi deve essere riconosciuto, infatti, è quello di strumenti attraverso i quali si può attuare una comparazione, in uno specifico ambito territoriale, tra il complesso delle trasformazioni, alterazioni, flussi e consumi di risorse - in atto o programmate - messe in gioco da un dato sistema di sviluppo e la capacità dell'ambiente e/o del paesaggio di "assorbire" tali variazioni e rigenerare le stesse risorse.

Gli indicatori, in buona sostanza, devono costituire uno strumento di supporto ai processi decisionali che intervengono su una data porzione di territorio e che riguardano un tema specifico; non devono essere pensati come elementi decisori in tale processo o, peggio, come soluzioni progettuali.

Un altro limite, poi, che si deve tener presente è che gli indicatori, di fatto, possono essere strumenti "statici", ovvero corrono il rischio - se mal utilizzati ed interpretati - di fotografare una data situazione nel presente e di proiettare nel futuro le possibili modifiche che avvengono 
a seguito di una specifica azione umana. Essi potrebbero, in sostanza, non dare

notizia del passato ${ }^{5}$,

che, al contrario, rappresenta una chiave di lettura indispensabile per comprendere l'evoluzione delle singole situazioni e per confrontare lo stato presente con quello più indietro nel tempo.

In estrema sintesi, gli indicatori possono essere visti come strumenti utili per:

- $\quad$ rappresentare in modo semplice problemi e realtà complessi;

- comprendere le correlazioni tra i diversi fenomeni locali e tra questi e quelli globali;

- identificare ed analizzare in modo sistematico i cambiamenti, le tendenze, i problemi prioritari, i rischi ambientali;

- $\quad$ supportare i processi decisionali, sia locali che sull'area vasta, da parte dei soggetti pubblici, così come di quelli provati;

- fare un bilancio delle azioni adottate e monitorarne l'efficacia nel medio e lungo periodo;

- aumentare la partecipazione locale, definendo obiettivi condivisi, e la collaborazione tra le comunità locali e i livelli superiori di governo.

Per selezionare, poi, gli indicatori adatti alla situazione nella quale ci si trova ad operare ci si deve basare sulle seguenti opzioni:

- la loro validità scientifica (attendibilità ed affidabilità dei metodi di misurazione; sensibilità ai mutamenti nel tempo dei fenomeni analizzati; capacità di mettere in evidenza le opportunità che

5 U. LEONE, Sugli indicatori di qualità ambientale, in MANZI E., SCHMIDT DI FRIEDBERG M. (a cura di), «Terra, ambienti, uomini: i geografi e gli indicatori ambientali», Marcos $Y$ Marcos, Milano 1996. 
devono essere valorizzate; comparabilità dei dati e delle stime effettuate nel tempo);

- l'applicabilità effettiva degli indicatori scelti (esistenza, reperibilità ed affidabilità del dati necessari; costi e tempi necessari alla raccolta ed elaborazione dati; disponibilità degli stessi dati ad essere raccolti in tempi diversi, nonché la loro frequenza di aggiornamento);

- la loro rilevanza ai fini dell'attivazione di politiche di sostenibilità e compatibilità (coerenza con gli obiettivi di qualità adottati in partenza; rappresentatività delle condizioni locali reali, dei fattori di pressione in atto sulle risorse esistenti, delle politiche urbane e territoriali intraprese);

- la loro adeguatezza ad orientare le decisioni e il comportamento dei decisori pubblici e privati (efficacia ed immediatezza comunicativa).

\section{INDICATORI ED AGRICOLTURA}

Come è stato detto, per essere significativi gli indicatori devono fornire un quadro il più preciso possibile dei processi e delle relazioni che collegano le attività umane all'ambiente. Ciò è ancor più vero nel caso dell'agricoltura, dove tale relazione è altamente complessa e dove la stessa attività agricola comprende e mette in atto una serie di processi che interessano in maniera sostanziale le risorse esistenti e una loro eventuale riproducibilità.

L'agricoltura, essendo innanzitutto un'attività economica, ha come scopo primario la produzione e si basa sulla disponibilità delle risorse naturali, anche se oggi (come si è già visto) ad essa vengono riconosciuti ruoli molteplici in termini di tutela dei paesaggi tradizionali e della biodiversità e sostenibilità degli ecosistemi, nonché delle identità delle comunità rurali. Gli indicatori che vengono applicati per analizzare lo stato di salute dell'agricoltura devono intenderla sia come fattore di 
produzione, che come fattore di tutela delle diversità ecosistemiche e paesistiche. È dunque necessario che nelle applicazioni pratiche sia contemplata l'elaborazione di indicatori che riflettano tanto gli effetti benefici, quanto quelli nocivi dell'agricoltura in entrambi i campi: produzione e tutela paesistico-ambientale.

Per elaborare una serie di indicatori utili e significativi, è dunque essenziale applicare un'impostazione differenziata, che tenga conto dei processi e delle strutture economiche sulle quali si basa l'agricoltura, insieme alle differenti condizioni paesistico-ambientali che disegnano il paesaggio agrario.

Inoltre è necessario che i dati degli indicatori riflettano il più possibile il quadro della situazione di una determinata zona e degli effetti esercitati dalle attività agricole locali: gli effetti di particolari processi inquinanti o di eventuali politiche di tutela e ripristino, infatti, dipenderanno da precise caratteristiche locali, specifiche di un luogo piuttosto che di un altro.

Un primo contesto che gli indicatori devono descrivere è, quindi, quello legato alle politiche e pratiche agricole: coniuga l'analisi dell'attività agricola vera e propria con lo studio (quantitativo) dei mezzi e dei fattori di produzione, del prezzo e della qualità dei prodotti, della produttività, del mercato e del progresso tecnologico delle aziende produttrici.

Un secondo ambito di analisi deve riguardare l'equilibrio tra l'attività agricola e l'ecosistema di cui questa fa parte, attraverso la comprensione della qualità dell'integrazione esistente tra le pratiche agricole - precedentemente analizzate in termini quantitativi - e l'ambiente e, quindi, della qualità del paesaggio come risultato di una corretta relazione fra le due parti in gioco. 


\section{ALCUNI ESEMPI}

I due esempi che seguono, presentati qui in maniera sintetica, rappresentano probabilmente le espressioni più compiute di elenchi di indicatori da utilizzare per valutare lo stato di salute di una determinata porzione di territorio.

Il primo esempio, il $\mathrm{V}^{\circ}$ volume del Manuale degli indicatori per la Valutazione di Impatto Ambientale curato dall'Associazione Analisti Ambientali insieme al Centro V.I.A. Italia e al FAST (Federazione delle Associazioni Scientifiche e Tecniche) nel 1999, è più attento ai fattori che interessano il paesaggio vero e proprio e agli indicatori attraverso i quali misurare le sue qualità (ecologico-ambientale, storico-insediativa e percettiva).

Il secondo esempio è il risultato al quale nel 2000 è giunta la Commissione Agricoltura dell'Unione Europea dopo una molteplicità di studi e ricerche (alcuni dei quali tutt'ora in corso), e mira innanzitutto a valutare l'incisività dell'agricoltura sull'ambiente, cercando di misurare quantitativamente gli effetti legati alla sua produttività e agli inquinamenti che causa.

\section{Manuale AAA degli indicatori per la Valutazione di Impatto Ambientale}

Lo sforzo maggiore del quale bisogna rendere merito a questo lavoro, è quello di aver mirato ad una sistematizzazione degli indicatori sul tema del paesaggio, in un ambito di studio che da sempre ha visto scontrarsi diverse teorie ed approcci sulla soggettività e oggettività di giudizio, ovvero sulla forbice che esiste tra gli aspetti qualitativi e gli aspetti quantitativi dell'analisi paesaggistica. 
Nel Manuale si è cercato di considerare le numerose scuole di pensiero, approntando una sorta di mediazione ${ }^{6}$ che tenesse in conto $\mathrm{i}$ valori evidenziati da ciascuna di esse:

* il valore puramente estetico (quale aspetto esteriore della bellezza 'artistica' dei luoghi);

* il valore insito principalmente nei beni storico/culturali (conservazione delle testimonianze : costruzioni, sistemazioni agrarie e infrastrutturali, "segni " storici e simbolici in generale );

* l'insieme geografico in continua trasformazione, con l'interazione degli aspetti naturalistici con quelli antropici (interrelazioni dinamiche significative connotanti i luoghi);

* i valori visivamente percepibili (caratteri della fruibilità del paesaggio, nelle sue proprietà sceniche, quale prodotto dell' individuo spettatore/attore). ${ }^{7}$

Gli indicatori che seguono sono stati articolati, per comodità e semplicità di lettura, secondo tre fattori - ecologici, storico-insediativi e percettivi - e sono desunti da quelli presenti nel Manuale che, al contrario, li aveva organizzati distinguendoli in 13 categorie: FisicoGeomorfologici, Fisico-Idrogeologici, Vegetazionali, Faunistici, Agricoli, Insediativi, Infrastrutturali, Storico-culturali, Percettivi generici, Percettivi da singoli punti di vista, Percettivi rispetto allinserimento di nuovi interventi, Ecosistemici strutturali, Ecosistemici funzionali.

6 II presente lavoro ha teso, come criterio generale, a non compiere scelte tra possibili approcci conflittuali delle diverse scuole di pensiero sulla materia, per puntare invece ad una raccolta sintetica degli indicatori generalmente rilevabili nella prassi corrente delle applicazioni negli studi di impatto ambientale, indipendentemente dalle possibili dissonanze riscontrabili.

(A.G. Colombo, S. Malcevschi (a cura di), Manuale AAA degli indicatori per la Valutazione di Impatto Ambientale, vol. 5, Centro Via Italia, AAA e Fast, Milano giugno 1999, p.3).

7 A.G. Colombo, S. Malcevschi (a cura di), op. cit., p.2. 


\section{$\underline{\text { I fattori ecologici }}$}

\begin{tabular}{|c|c|c|}
\hline $\begin{array}{l}\text { Elementi caratteristici del } \\
\text { rilievo }\end{array}$ & $\begin{array}{l}\text { Sono rappresentati dalle principali } \\
\text { caratteristiche fisico- } \\
\text { geomorfologiche che determinano il } \\
\text { paesaggio, quali ad esempio } \\
\text { - le sommità collinari, } \\
\text { - i versanti, } \\
\text { - le emergenze strutturali della } \\
\text { pianura, } \\
\text { - i terrazzi } \\
\text { - i dossi e i rilievi. }\end{array}$ & \\
\hline $\begin{array}{l}\text { Elementi caratteristici } \\
\text { dell'idrografia }\end{array}$ & $\begin{array}{l}\text { Sono rappresentati dalle principali } \\
\text { caratteristiche idrogeologiche che } \\
\text { determinano il paesaggio e sono } \\
\text { riferiti agli aspetti significativi del } \\
\text { paesaggio naturale generati dalla } \\
\text { presenza delle acque; ad esempio } \\
\text { sono } \\
\text { - i laghi, } \\
\text { - i fiumi, } \\
\text { - i torrenti, } \\
\text { - le forre, } \\
\text { - le spiagge. }\end{array}$ & \\
\hline $\begin{array}{l}\text { Elementi caratteristici della } \\
\text { copertura vegetazionale }\end{array}$ & $\begin{array}{l}\text { Definiscono tipologicamente, } \\
\text { qualitativamente e } \\
\text { quantitativamente i principali } \\
\text { caratteri delle componenti } \\
\text { ambientali relative alla vegetazione } \\
\text { e, in genere, sono } \\
\text { - i boschi, } \\
\text { - le macchie boschive, } \\
\text { - la vegetazione ripariale, } \\
\text { - la flora protetta, } \\
\text { - i filari arborei e le siepi, } \\
\text { - i grandi alberi isolati. } \\
\end{array}$ & \\
\hline $\begin{array}{l}\text { Elementi caratteristici della } \\
\text { connettività }\end{array}$ & $\begin{array}{l}\text { Indici utilizzati per comprendere le } \\
\text { dinamiche paesistiche e la stabilità } \\
\text { delle strutture. Misurano le effettive } \\
\text { possibilità di scambi funzionali } \\
\text { all'interno delle strutture paesistiche, } \\
\text { mettendo in relazione i legami che } \\
\text { intercorrono tra macchie e corridoi. }\end{array}$ & \multirow[t]{2}{*}{$\begin{array}{l}\text { Connettività e } \\
\text { circuitazione } \\
\text { possono essere } \\
\text { utilizzati insieme per } \\
\text { la valutazione della } \\
\text { funzionalità delle } \\
\text { strutture } \\
\text { paesistiche. }\end{array}$} \\
\hline $\begin{array}{l}\text { Elementi caratteristici della } \\
\text { circuitazione }\end{array}$ & $\begin{array}{l}\text { Misurano l'efficienza dei sistemi a } \\
\text { rete. }\end{array}$ & \\
\hline
\end{tabular}

8 Gli aspetti floristici e vegetazionali possono rientrare sia nello studio delle componenti ecosistemiche, sia nell'individuazione di significati simbolici del paesaggio. 


\begin{tabular}{|l|l|l|}
\hline $\begin{array}{l}\text { Elementi caratteristici } \\
\text { dell'eterogeneità }\end{array}$ & $\begin{array}{l}\text { Misurano il grado di eterogeneità in } \\
\text { relazione alla capacità di } \\
\text { mantenimento dell'equilibrio }\end{array}$ \\
& $\begin{array}{l}\text { complessivo dei sistemi paesistici. } \\
\text { Sono indici solitamente applicati agli } \\
\text { ecotopi o alle singole macchie, per i } \\
\text { quali viene considerata la superficie } \\
\text { occupata, anziché il numero di } \\
\text { individui. }\end{array}$ & \\
\hline
\end{tabular}

\section{I fattori storico - insediativi}

\begin{tabular}{|c|c|c|}
\hline $\begin{array}{l}\text { Elementi caratterizzanti la } \\
\text { tessitura delle coltivazioni }{ }^{9}\end{array}$ & $\begin{array}{l}\text { Partecipano alla definizione di } \\
\text { tipologie significative delle principali } \\
\text { organizzazioni delle colture presenti } \\
\text { in un determinato territorio, } \\
\text { rappresentando degli elementi } \\
\text { fondamentali per determinare la } \\
\text { qualità del paesaggio. } \\
\text { Rientrano fra questi elementi } \\
\text { - i terrazzamenti, } \\
\text { - i campi piani. }\end{array}$ & \\
\hline $\begin{array}{l}\text { Elementi caratterizzanti il } \\
\text { frazionamento fondiario }\end{array}$ & $\begin{array}{l}\text { Fra i diversi tipi di frazionamento } \\
\text { fondiario si possono indicare come } \\
\text { esempi } \\
\text { - la maglia derivante dalla } \\
\text { centuriazione romana, } \\
\text { - la maglia frammentata, } \\
\text { - la maglia spontanea, } \\
\text { - la maglia a poderi. }\end{array}$ & $\begin{array}{l}\text { A caratterizzare un } \\
\text { particolare } \\
\text { frazionamento } \\
\text { contribuiscono in } \\
\text { maniera } \\
\text { preponderante gli } \\
\text { elementi } \\
\text { componenti la } \\
\text { maglia irrigua. }\end{array}$ \\
\hline $\begin{array}{l}\text { Elementi caratterizzanti i tipi } \\
\text { di colture prevalenti }\end{array}$ & $\begin{array}{l}\text { Sono riferiti alle componenti vegetali } \\
\text { delle colture e alle loro tipologie } \\
\text { significative per le qualità del } \\
\text { paesaggio agrario. } \\
\text { Alcuni esempi sono } \\
\text { - le coltivazioni legnose, } \\
\text { - i seminativi arborei, } \\
\text { - i seminativi semplici, } \\
\text { - i prati, } \\
\text { - i pascoli, } \\
\text { - i boschi d'impianto, } \\
\text { - le alberature e le siepi poderali. }\end{array}$ & $\begin{array}{l}\text { Le diverse forme di } \\
\text { organizzazione e } \\
\text { connotazione } \\
\text { territoriale che gli } \\
\text { usi agricoli } \\
\text { comportano } \\
\text { caratterizzano in } \\
\text { maniera consistente } \\
\text { l'immagine } \\
\text { paesistica di un } \\
\text { luogo. }\end{array}$ \\
\hline
\end{tabular}

9 Gli indicatori in oggetto sono considerati sia componenti connotative per l'individuazione di unità paesistiche, sia riferimenti per l'analisi percettiva. 


\begin{tabular}{|c|c|}
\hline $\begin{array}{l}\text { Elementi caratteristici le } \\
\text { strutture annesse all'attività } \\
\text { agricola }\end{array}$ & $\begin{array}{l}\text { Sono riferiti alle componenti } \\
\text { insediative annesse all'attività } \\
\text { agricola significative per le qualità } \\
\text { del paesaggio agrario. } \\
\text { Fra le varie possibilità si possono } \\
\text { ricordare } \\
\text { - le cascine tradizionali, } \\
\text { - i casali di bonifica, } \\
\text { - le ville suburbane, } \\
\text { - i fienili tradizionali. }\end{array}$ \\
\hline $\begin{array}{l}\text { Elementi caratterizzanti i tipi } \\
\text { di urbanizzazione }\end{array}$ & $\begin{array}{l}\text { Sono riferiti alla qualità degli } \\
\text { insediamenti umani. } \\
\text { Rappresentano le principali } \\
\text { caratteristiche degli insediamenti } \\
\text { agricoli che determinano il } \\
\text { paesaggio che, solitamente, } \\
\text { possono essere distinti in } \\
\text { - urbanizzazione spontanea, } \\
\text { - urbanizzazione diffusa. }\end{array}$ \\
\hline $\begin{array}{l}\text { Elementi caratteristici per la } \\
\text { collocazione } \\
\text { dell'insediamento }\end{array}$ & $\begin{array}{l}\text { Sono riferiti all'organizzazione degli } \\
\text { insediamenti umani che possono } \\
\text { essere collocati } \\
\text { - in pianura, } \\
\text { - sulla sommità di un'altura, } \\
\text { - sui versanti, } \\
\text { - lungo le riviere. }\end{array}$ \\
\hline $\begin{array}{l}\text { Elementi caratterizzanti il } \\
\text { disegno storico degli } \\
\text { insediamenti }\end{array}$ & $\begin{array}{l}\text { Sono riferiti alle caratteristiche che } \\
\text { disegnano la struttura storica degli } \\
\text { insediamenti, come } \\
\text { - i centri storici (di origine romana, } \\
\text { medievale, rinascimentale, ...), } \\
\text { - le aree edificate derivate dalla } \\
\text { centuriazione romana, } \\
\text { - le aree edificate derivate dalle } \\
\text { opere di bonifica otto e } \\
\text { novecentesche. }\end{array}$ \\
\hline $\begin{array}{l}\text { Elementi di interesse } \\
\text { archeologico e storico- } \\
\text { monumentale }\end{array}$ & $\begin{array}{l}\text { Sono riferiti a tutti i principali beni } \\
\text { storico-culturali in grado di } \\
\text { connotare significativamente il } \\
\text { paesaggio, come } \\
\text { - i ruderi, } \\
\text { - i castelli, le torri e le rocche, } \\
\text { - le chiese, le abbazie e le pievi. }\end{array}$ \\
\hline
\end{tabular}




\begin{tabular}{|c|c|c|}
\hline $\begin{array}{l}\text { Elementi caratteristici delle } \\
\text { infrastrutture della mobilità e } \\
\text { delle infrastrutture lungo corsi } \\
\text { d'acqua }\end{array}$ & $\begin{array}{l}\text { Prendono in considerazione tutti } \\
\text { quei caratteri delle infrastrutture } \\
\text { territoriali e degli impianti a rete che } \\
\text { vengono considerati elementi } \\
\text { costitutivi del paesaggio. } \\
\text { Alcuni di questi elementi sono } \\
\text { - i sentieri, } \\
\text { - i percorsi e i punti panoramici, } \\
\text { - i tracciati storici, } \\
\text { - le strade, } \\
\text { - le ferrovie, } \\
\text { - i canali, } \\
\text { - il sistema irriguo minore, } \\
\text { - le arginature, } \\
\text { - le sistemazioni idrauliche. }\end{array}$ & $\begin{array}{l}\text { II disegno territoriale } \\
\text { delle infrastrutture } \\
\text { ha storicamente } \\
\text { strutturato il } \\
\text { territorio e } \\
\text { costituisce un } \\
\text { elemento importante } \\
\text { di connotazione del } \\
\text { paesaggio }{ }^{10} \text {. }\end{array}$ \\
\hline
\end{tabular}

\section{I fattori percettivi}

\begin{tabular}{|c|c|c|}
\hline $\begin{array}{l}\text { Elementi strutturanti le } \\
\text { immagini ambientali }\end{array}$ & $\begin{array}{l}\text { Sono riferiti a tutti quegli elementi } \\
\text { attraverso i quali identificare gli } \\
\text { aspetti significativi dei rapporti } \\
\text { morfologici e scenici del paesaggio. } \\
\text { Alcuni di questi elementi sono } \\
\text { - i percorsi, ovvero i canali lungo i } \\
\text { quali l'osservatore si muove } \\
\text { abitualmente; } \\
\text { - i margini, ossia gli elementi lineari } \\
\text { che solitamente non vengono } \\
\text { considerati ed usati come } \\
\text { percorsi dall'osservatore; } \\
\text { - i nodi, cioè i luoghi verso i quali o } \\
\text { dai quali l'osservatore si muove, } \\
\text { punti di congiunzione di percorsi } \\
\text { e punti di concentra-zione di } \\
\text { qualche attività o caratteristica } \\
\text { fisica; } \\
\text { - i riferimenti, ovvero altri elementi } \\
\text { "puntiformi" come i nodi, in cui } \\
\text { però l'osservatore non entra e } \\
\text { che fungono da riferimento. }\end{array}$ & $\begin{array}{l}\text { K. Linch ha, ad } \\
\text { esempio, utilizzato } \\
\text { alcuni di questi } \\
\text { elementi per } \\
\text { descrivere quartieri } \\
\text { di alcune città } \\
\text { americane (Boston, } \\
\text { Los Angeles, Jersey } \\
\text { city). }\end{array}$ \\
\hline $\begin{array}{l}\text { Elementi per la valutazione } \\
\text { della qualità visiva da parte } \\
\text { del pubblico }\end{array}$ & $\begin{array}{l}\text { Servono a definire il livello di } \\
\text { gradimento di un paesaggio da } \\
\text { parte dei suoi fruitori; in particolare } \\
\text { - dei residenti, } \\
\text { - dei fruitori provenienti } \\
\text { dall'esterno. }\end{array}$ & $\begin{array}{l}\text { Il giudizio sul livello } \\
\text { di gradimento di un } \\
\text { paesaggio può } \\
\text { consentire di } \\
\text { stabilire il consenso } \\
\text { sociale sui suoi } \\
\text { contenuti } \\
\text { visivamente } \\
\text { percepibili. }\end{array}$ \\
\hline
\end{tabular}

\footnotetext{
10 Su questi temi cfr. capitolo 1.
} 


\begin{tabular}{|c|c|c|}
\hline $\begin{array}{l}\text { Elementi caratteristici } \\
\text { dell'efficacia generale della } \\
\text { percezione }\end{array}$ & $\begin{array}{l}\text { Descrivono la tipologia della } \\
\text { percezione, analizzando la } \\
\text { posizione o situazione in cui si trova } \\
\text { l'oggetto che viene percepito } \\
\text { rispetto al soggetto che percepisce } \\
\text { - in primo piano, } \\
\text { - sullo sfondo, } \\
\text { - difficilmente percepibile. }\end{array}$ & \\
\hline Detrattori visivi & $\begin{array}{l}\text { Sono riferiti a tutti quegli elementi la } \\
\text { cui presenza o assenza concorre a } \\
\text { definire gli aspetti qualitativi del } \\
\text { paesaggio. } \\
\text { Alcuni di questi elementi, per } \\
\text { esempio, sono } \\
\text { - discariche, } \\
\text { - rifiuti, } \\
\text { - fronti di cava, } \\
\text { - elementi di dequalificazione. }\end{array}$ & \\
\hline $\begin{array}{l}\text { Elementi descrittori del grado } \\
\text { di intrusione visiva }\end{array}$ & $\begin{array}{l}\text { Definiscono l'impatto di un nuovo } \\
\text { intervento, visto da un punto (o da } \\
\text { una serie di punti) di osservazione } \\
\text { predeterminato rispetto al contesto } \\
\text { in cui l'opera è collocata. } \\
\text { Sono, ad esempio } \\
\text { - la distanza dell'oggetto osservato } \\
\text { rispetto al soggetto percepente, } \\
\text { - l'ingombro fisico del nuovo } \\
\text { intervento, } \\
\text { - la frequenza con cui il nuovo } \\
\text { oggetto viene osservato. }\end{array}$ & $\begin{array}{l}\text { Costituiscono degli } \\
\text { indicatori da } \\
\text { utilizzare nel caso di } \\
\text { inserimenti di nuovi } \\
\text { interventi. }\end{array}$ \\
\hline $\begin{array}{l}\text { Elementi caratteristici per la } \\
\text { qualità dell'intrusione visiva }\end{array}$ & $\begin{array}{l}\text { Descrivono i rapporti visivi esistenti } \\
\text { tra gli interventi in progetto ed il } \\
\text { paesaggio e ne individuano il grado } \\
\text { di compatibilità. } \\
\text { I rapporti possono presentarsi sotto } \\
\text { diverse forme: } \\
\text { - insignificanza, } \\
\text { - armonia, } \\
\text { - contrasto. }\end{array}$ & $\begin{array}{l}\text { Costituiscono degli } \\
\text { indicatori da } \\
\text { utilizzare nel caso di } \\
\text { inserimenti di nuovi } \\
\text { interventi. }\end{array}$ \\
\hline $\begin{array}{l}\text { Elementi descrittori del grado } \\
\text { di variazione della qualità } \\
\text { paesaggistica complessiva }\end{array}$ & $\begin{array}{l}\text { Sono utilizzati per definire le } \\
\text { modifiche del nuovo intervento sul } \\
\text { significato e sui valori del paesaggio } \\
\text { individuati nell'unità paesistica prima } \\
\text { dell'intervento. } \\
\text { I giudizi vengono compiuti su: } \\
\text { - perdita di beni ambientali, } \\
\text { - perdita della connotazione } \\
\text { caratteristica dei luoghi, } \\
\text { - peggioramento (o miglioramento) } \\
\text { della qualità dell'unità paesistica, } \\
\text { - aumento (o riduzione) del } \\
\text { degrado visivo. }\end{array}$ & $\begin{array}{l}\text { Costituiscono degli } \\
\text { indicatori da } \\
\text { utilizzare nel caso di } \\
\text { inserimenti di nuovi } \\
\text { interventi. }\end{array}$ \\
\hline
\end{tabular}


Gli indicatori per l'integrazione della problematica ambientale nella Politica Agricola Comune proposti dalla Commissione Agricoltura dell'Unione Europea

Sin dalle riforme intraprese dall'Unione Europea nell'ambito del progetto comunitario "Agenda 2000", vi è stata un'attenzione particolare all'integrazione della problematica ambientale nella politica agricola ${ }^{11}$.

Gli indicatori che qui vengono riassunti si basano soprattutto sui lavori dell'OCSE e sui contributi complementari dell'Eurostat, dell'Agenzia europea dell'ambiente e del progetto di ricerca ELISA. ${ }^{12}$

A seguito di questi studi, la maggiore attenzione della UE è stata incentrata all'utilizzo degli indicatori per ciò che riguarda la valutazione ambientale dell'agricoltura e della sua produttività in termini di sviluppo sostenibile. Lo schema metodologico adottato ha messo al centro delle analisi la situazione attuale dell'ambiente agricolo ed i suoi mutamenti nel tempo, con l'utilizzo di indicatori attraverso i quali sono state messe in rilievo sia le possibili modifiche indesiderabili da evitare, sia le condizioni esistenti da salvaguardare. In una seconda fase sono state, poi, individuate le pressioni che hanno portato ai mutamenti indesiderabili, insieme ai benefici che determinate coltivazioni agricole hanno contribuito a generare. La terza ed ultima fase ha visto il

11 Questi temi sono stati trattati dettagliatamente nel capitolo 2.

12 L'OCSE ha individuato tredici settori tematici, sia legati ai possibili fattori di inquinamento, sia legati ai fattori più legati ad aspetti produttivi, ed ha elaborato una serie di indicatori contestuali riguardanti la copertura vegetale e l'uso del suolo.

L'Eurostat, in quanto Istituto Statistico ufficiale della UE, raccoglie le statistiche di base sull'agricoltura e le struttura in tre settori tematici (struttura delle aziende agricole, patrimonio animale e produzione vegetale, conti economici dell'agricoltura). L'Agenzia europea dell'ambiente pur non essendosi occupata specificatamente di indicatori agroambientali, molti dei suoi lavori relativi a questi temi possono essere applicati a questo settore di ricerca.

Il progetto di ricerca ELISA, condotto dal Centro europeo per la conservazione della natura, aveva come obiettivo quello di migliorare la coerenza territoriale dei lavori già sviluppati dall'OCSE; per far questo sono stati individuati 22 indicatori di situazione (che hanno studiato il suolo, le risorse idriche, l'atmosfera, la biodiversità, i paesaggi) e sono stati descritti 12 indicatori di pressione (che hanno analizzato le diverse partiche agricole e i loro potenziali effetti).

(Dal sito europa.eu.int/comm/environment/newprg) 
collegamento delle pressioni e dei processi suddetti alle forze motrici dell'economia, in quanto è principalmente a queste che si lega un mantenimento o meno degli spazi dedicati all'agricoltura.

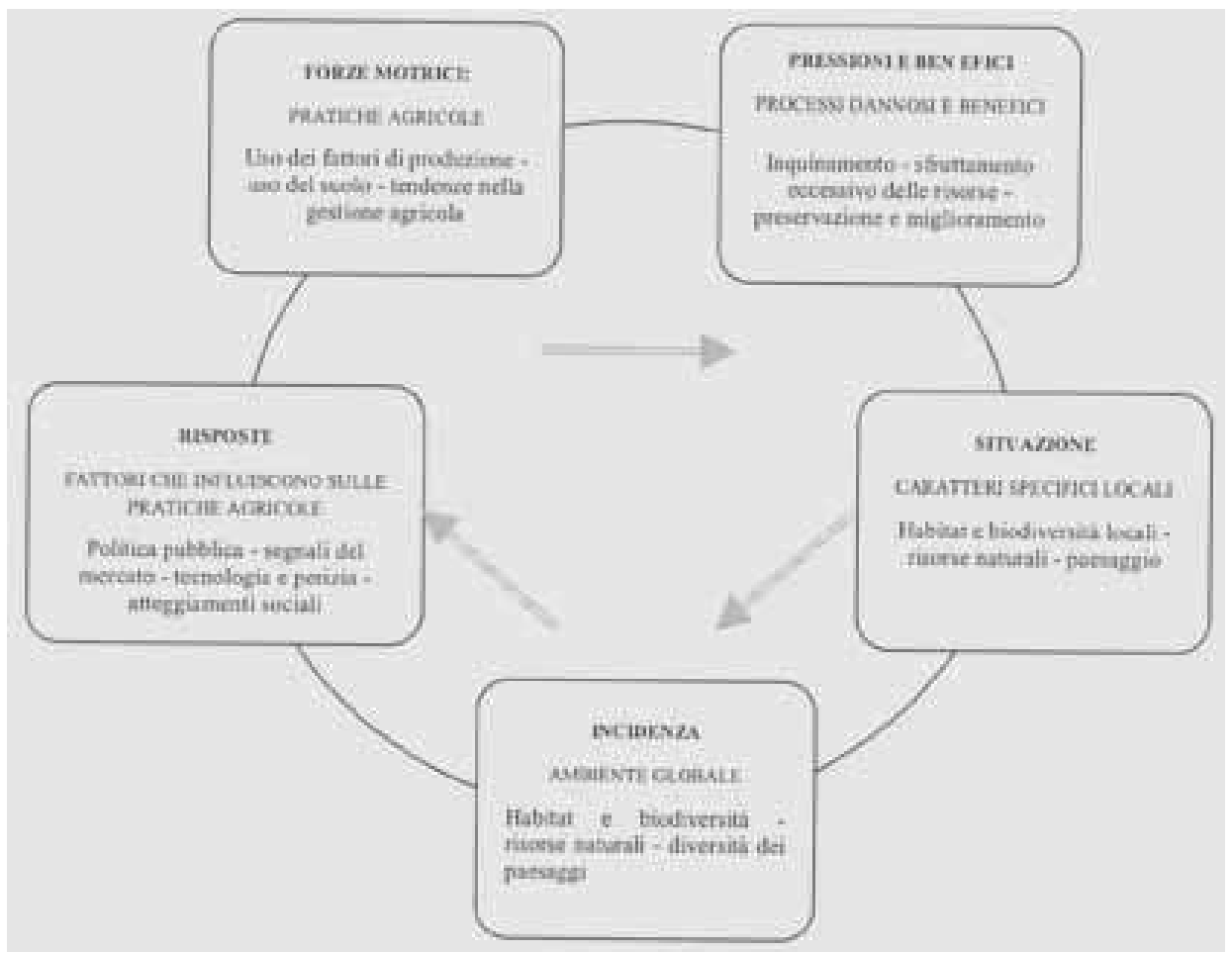

Figura 10. II modello agricolo DPSIR (forze motrici, pressioni, situazioni, incidenza, risposta) utilizzato negli studi dell'Unione Europea.

\section{$\underline{\text { I fattori e le risposte che influiscono sulla pratiche agricole }}$}

\begin{tabular}{|l|l|}
\hline Politiche pubbliche & $\begin{array}{l}\text { - area oggetto dei programmi } \\
\text { agroambientali, } \\
\text { - area protetta, } \\
\bullet\end{array}$ \\
& $\begin{array}{l}\text { livelli regionali di buone pratiche } \\
\text { agricole, }\end{array}$ \\
\hline Segnali del mercato & $\begin{array}{l}\text { - premio per i prezzi ai produttori } \\
\text { biologici. }\end{array}$ \\
\hline Tecnologie & $\begin{array}{l}\text { livelli di formazione dei conduttori } \\
\text { agricoli. }\end{array}$ \\
\hline
\end{tabular}


collegamento delle pressioni e dei processi suddetti alle forze motrici dell'economia, in quanto è principalmente a queste che si lega un mantenimento o meno degli spazi dedicati all'agricoltura.

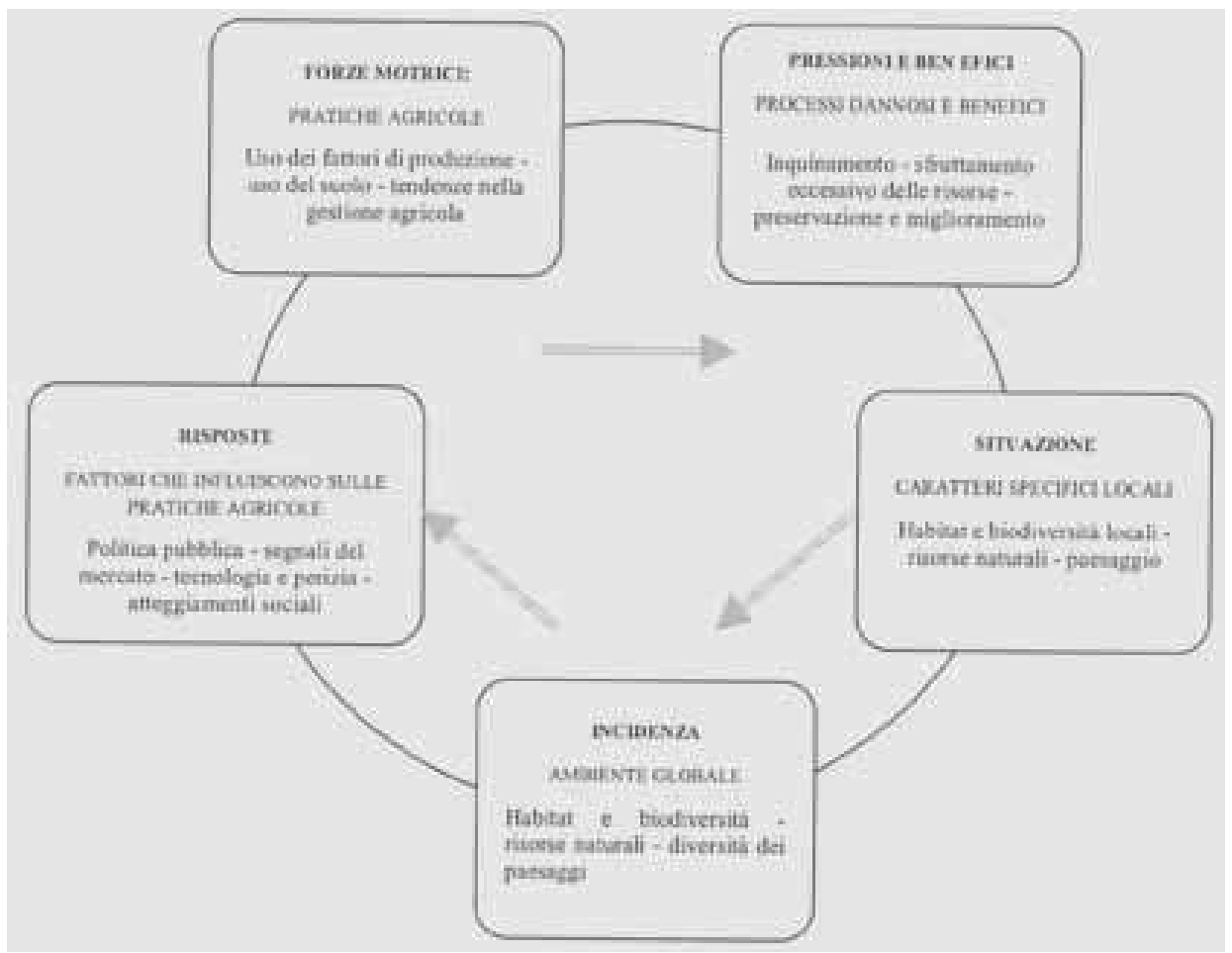

Figura 10. II modello agricolo DPSIR (forze motrici, pressioni, situazioni, incidenza, risposta) utilizzato negli studi dell'Unione Europea.

\section{$\underline{\text { I fattori e le risposte che influiscono sulla pratiche agricole }}$}

\begin{tabular}{|l|l|}
\hline Politiche pubbliche & $\begin{array}{l}\text { - area oggetto dei programmi } \\
\text { agroambientali, } \\
\text { - area protetta, } \\
\bullet\end{array}$ \\
& $\begin{array}{l}\text { livelli regionali di buone pratiche } \\
\text { agricole, }\end{array}$ \\
\hline Segnali del mercato & $\begin{array}{l}\text { - premio per i prezzi ai produttori } \\
\text { biologici. }\end{array}$ \\
\hline Tecnologie & $\begin{array}{l}\text { - livelli di formazione dei conduttori } \\
\text { agricoli. }\end{array}$ \\
\hline
\end{tabular}




\section{Le caratteristiche delle pratiche agricole}

\begin{tabular}{|c|c|}
\hline $\begin{array}{l}\text { Impiego di fattori di } \\
\text { produzione }\end{array}$ & $\begin{array}{l}\text { - consumo medio di fertilizzanti, } \\
\text { - consumo medio di pesticidi, } \\
\text { - intensità di impiego delle risorse } \\
\text { idriche, } \\
\text { - intensità di impiego delle risorse } \\
\text { energetiche. }\end{array}$ \\
\hline Uso del suolo & $\begin{array}{l}\text { - mutamento topologico, } \\
\text { - sistemi di coltivazione e di } \\
\text { allevamento. }\end{array}$ \\
\hline Gestione & $\#$ \\
\hline Tendenze & $\begin{array}{l}\text { - intensificazione, } \\
\text { - estensificazione, } \\
\text { - specializzazione, } \\
\text { - diversificazione, } \\
\text { - marginalizzazione. }\end{array}$ \\
\hline
\end{tabular}

\section{I processi nocivi e i processi benefici}

\begin{tabular}{|c|c|}
\hline Inquinamento & $\begin{array}{l}\text { - equilibrio dell'azoto sulla superficie del } \\
\text { suolo, } \\
\text { - contaminazione del suolo a causa } \\
\text { dell'uso di pesticidi, } \\
\text { - contaminazione delle risorse idriche. }\end{array}$ \\
\hline $\begin{array}{l}\text { Sfruttamento eccessivo delle } \\
\text { risorse }\end{array}$ & $\begin{array}{l}\text { - estrazione di acque profonde e } \\
\text { pressioni sulle risorse idriche, } \\
\text { - erosione del suolo, } \\
\text { - distruzione della copertura vegetale, } \\
\text { - introduzione di specie a bassa } \\
\text { diversità genetica. }\end{array}$ \\
\hline Benefici & $\begin{array}{l}\text { - } \text { presenza di elevati valori naturali: } \\
\text { copertura erbosa, ecc..., } \\
\text { - produzione di fonti energetiche } \\
\text { rinnovabili. }\end{array}$ \\
\hline
\end{tabular}

\section{La situazione specifica locale}

\begin{tabular}{|l|l|}
\hline Habitat & $\begin{array}{l}\bullet \text { ricchezza della specie (ricchezza } \\
\text { avicola). }\end{array}$ \\
\hline Riodiversità & $\begin{array}{l}\bullet \text { qualità del suolo, } \\
\text { nitrati/pesticidi nelle acque superficiali } \\
\text { e profonde, }\end{array}$ \\
\hline Paesaggio & livelli della falda freatica. \\
\hline
\end{tabular}


L'incidenza globale

\begin{tabular}{|l|l|}
\hline $\begin{array}{l}\text { Habitat } \\
\text { Biodiversità }\end{array}$ & $\#$ \\
\hline Risorse naturali & $\begin{array}{l}\bullet \text { percentuale dell'agricoltura nelle } \\
\text { emissioni, }\end{array}$ \\
& $\begin{array}{l}\bullet \text { contaminazione da nitrati, } \\
\bullet \text { uso delle risorse idriche. }\end{array}$ \\
\hline Diversità del paesaggio & $\#$ \\
\hline
\end{tabular}

In alcuni settori (qui contrassegnati con il simbolo "\#”) I'Unione Europea non è ancora stata in grado di definire una serie di indicatori operativi adeguati. Pur disponendo di una considerevole mole di dati che riguardano i fattori e le risposte che influiscono sulle pratiche agricole, le ricerche in corso stanno lavorando su questi elementi per ottenere una serie più completa di indicatori il più coerenti possibile con le problematiche poste. 


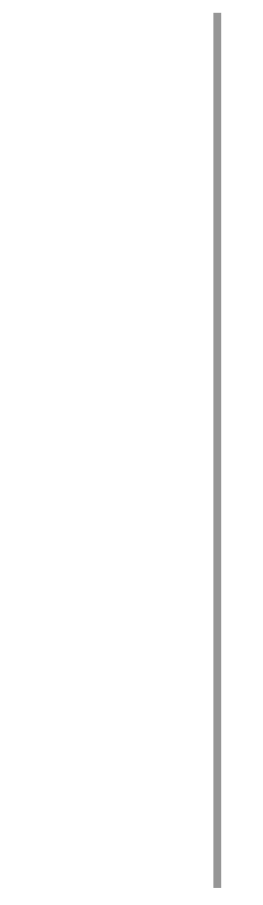

PARTE PRIMA

BIBLIOGRAFIA RAGIONATA DI RIFERIMENTO

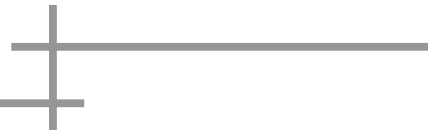


Farsi una bibliografia significa cercare quello di cui non si conosce ancora l'esistenza.'

\section{SULLA LOGICA DI STRUTTURA}

- AA.VV., Carta di Napoli. Il parere degli specialisti sulla riforma degli ordinamenti di tutela del paesaggio in Italia, Napoli 1999.

- Assunto Rosario, Paesaggio, ambiente, territorio: un tentativo di precisazione concettuale, in «Rassegna di architettura e urbanistica» nn.47-48, ago.-dic., Kappa, Roma 1980, pp.49-51.

- Beltrame G., Interpretare e rappresentare il paesaggio, in AA.VV., "Opinioni sul paesaggio», Ordine degli architetti della Provincia di Como, Como 2000, pp.9-15.

- Calzolari V., Concetto di paesaggio e paesistica, in AA.VV., «Architettura del paesaggio», La Nuova Italia, Firenze 1975, pagg. 73-85.

- CARBonara L., I paesaggi naturali: analisi e pianificazione, in «Rassegna di architettura e urbanistica» nn.47-48, ago.-dic., Kappa, Roma 1980, pp.145-175.

- Cauquelin A., L'invention du paysage, Plon, Paris 1989.

- Corboz P., II territorio come palinsesto, in «Casabella» n. 516, Elemond periodici, Milano 1985, pp.22-27.

- Falini P., Bruschi S., Il paesaggio, in GisotTI G., Bruschi S. (a cura di), «Valutare l'ambiente», NIS, Roma 1990.

- GAMBI L., I valori storici dei quadri ambientali, in «Storia d'Italia: i caratteri originali», vol.1, Einaudi, Torino 1968, pp.5-60.

- Gambino R., Maniere di intendere il paesaggio, in ClemENTI A. (a cura di), «Interpretazioni di paesaggio», Meltemi, Roma 2002, pp.5472.

- Gregotti V. (a cura di), La forma del territorio, in «Edilizia moderna» nn.87-88, Roma 1965.

- Monti C., Pratelli A., Riguzzi G., Secondini P., Analisi e pianificazione del territorio rurale, Clueb, Bologna 1985.

1 U. Eco, Come si fa una tesi di laurea. Le materie umanistiche, Bompiani, Milano 1977, p.66. 
- Olivieri M., Come leggere il territorio, La Nuova Italia, Scandicci 1987.

- Pignatti S., Paesaggio vegetazionale e paesaggio agrario, in «Casabella» n.575-576, gen.-feb., Elemond periodici, Milano 1991, pp.73-76.

- Rizzo G. G. (a cura di), Tuscia Romana. Paesaggio e Territorio. Metodi e modelli di valutazione, Gangemi, Roma 1990.

- Romani V., Paesaggio Teoria e pianificazione, Franco Angeli, Milano 1994.

- TuRRI E., La lettura del paesaggio, in «Piano Progetto Città» n.16, lug.-dic. Pescara 1998, pp.30-33.

- Vascelli Vallara U., Concetto di paesaggio, il ruolo dell'esperto, in AA.VV., «Opinioni sul paesaggio», Ordine degli architetti della Provincia di Como, Como 2000, pp.27-33.

- Zerbi M.C., Paesaggi della geografia, Giappichelli, Torino 1993.

\section{SUGLI ELEMENTI COMPONENTI E SULLE LORO RELAZIONI}

- BLoch M., Les caractères originaux de l'histoire rurale française, Parigi 1953.

- Bortolotti L., Storia, città e territorio, Franco Angeli, Milano1993.

- CAmbi F., TerRenato N., Introduzione all'archeologia dei paesaggi, NIS, Roma 1994.

- Castiglioni C.B., Geomorfologia, UTET, Torino s.d.

- Cecchetto A., Archeologia rurale e variazioni tipologiche, in «Urbanistica» n.86, marzo, Franco Angeli, Milano 1987, pp.10-19.

- FABBRI P., Natura e cultura del paesaggio agrario: indirizzi per la tutela e la progettazione, Città Studi, Milano 1997.

- FabBri P. (a cura di), Paesaggio, pianificazione, sostenibilità, Alinea, Firenze 2003.

- Falini P., GRIFONI C., LOMORO R.A., Strutture agrarie storiche e fonti catastali geodetiche: alcune questioni e proposte di metodo, in MARTINELLI R., NUTI L., «Fonti per lo studio del paesaggio agrario», Atti del $3^{\circ}$ Convegno di Storia urbanistica, Lucca 3-5 ottobre 1979. 
- FALINI P., I paesaggi rurali: storia e progettazione, in «Rassegna di architettura e urbanistica»nn.47-48, ago.-dic., Kappa, Roma 1980, pp.52-75.

- Falini P., Grifoni C., Lomoro R.A., Tipologie agrarie storiche e pianificazione territoriale, in «Agricoltura Ambiente» n.11, giu., F.lli Palombi, Roma 1981, pp.27-47.

- FALINI P., CIARDINI F., II paesaggio rurale: qualità e sensibilità trasformative, in M. OTTOLENGHI (a cura di), «Territorio e ambiente: da Palidoro a Montalto di Castro», Roma 1986, pp.89-118.

- FAuCher D., Géographie agraire, Parigi 1949.

- Ferrara G., L'architettura del paesaggio italiano, Marsilio, Padova 1968.

- GamBı L., Per una storia dell'abitazione rurale in Italia, in «Rivista Storica Italiana» n.2, a.LXXVI, 1964, pp.427-453.

- George P., Manuale di geografia rurale, Edizioni di Comunità, Milano 1972.

- Gisotti G., Geologia e pedologia nell'assetto del territorio, Ed. agricole, Bologna 1983.

- Gli insediamenti rurali nel paesaggio agrario, in «Paesaggio Urbano» n.1, gen-feb., Maggioli, Rimini 1995.

- Malassis L., Passato e divenire dei paesaggi agricoli, in «Paesaggio mediterraneo», Electa, Milano 1992, pp.266-273.

- Marinelli O., Atlante dei tipi geografici, pubblicazione a cura dell'Istituto Geografico Militare, Firenze 1949.

- Marocco M., Il progetto dello spazio rurale, in «Rassegna di architettura e urbanistica» nn.47-48, ago.-dic., Kappa, Roma 1980, pp.76-96.

- Merlo M., Agricoltura, foreste e territorio. La formazione dei paesaggi rurali italiani e l'evoluzione nell'uso del territorio, in FABBRI M. (a cura di), «Pianificazione del territorio agricolo», INVET / Franco Angeli, Milano 1986, pp.13-39.

- Monti C., Pratelli A., Riguzzi G., Secondini P., Analisi e pianificazione del territorio rurale, Clueb, Bologna 1985.

- Natali C., Risorse e analisi del territorio, Alinea, Firenze 1998.

- PARDI F., L'osservazione geomorfologica del paesaggio, in MAGNAGH A. (a cura di), «Rappresentare i luoghi: metodi e tecniche», Alinea, Firenze 2001, pp.139-212.

- Pierotti P., Introduzione all'ecostoria, Franco Angeli, Milano 1982. 
- POLI D., Rappresentazione delle identità storico-morfologiche dei luoghi, in MAGNAGHI A. (a cura di), «Rappresentare i luoghi: metodi e tecniche», Alinea, Firenze 2001, pp.215-286.

- Rizzo B.M., Paesaggio rurale: verso quali modelli e con quali strumenti? Risposte urbanistiche attuali alle trasformazioni del paesaggio italiano, Tesi di dottorato in «Progettazione urbana territoriale ed ambientale» - X ciclo, Firenze 1998.

- Sartore M., Forme e processi di urbanizzazione diffusa. Un'analisi della morfologia insediativa in un'area rurale del veneto centrale, in «Archivio di studi urbani e regionali» n.32, Franco Angeli, Milano 1988, pp.165-218.

- Sereni E., Storia del paesaggio agrario italiano, Laterza, Roma-Bari 1961; nuova ed. 1999.

- Sestini A., Introduzione allo studio dell'ambiente. Fondamenti di geografia fisica, Franco Angeli, Milano 1983.

- Soprintendenza Archeologica di Roma, Misurare la terra: centuriazione e coloni nel mondo romano: città, agricoltura, commercio: materiali da Roma e dal suburbio [Roma: aprile - giugno 1985: catalogo della mostra], Panini edizioni, Modena 1985.

- ZANGHERI R., Agricoltura e contadini nella storia d'Italia, Einaudi, Torino 1977.

\section{SULLE TRASFORMAZIONI SUBITE DAL PAESAGGIO AGRARIO}

- BAsile E., CeCCHI C., La trasformazione post-industriale della campagna, Rosenberg \& Sellier, Torino 2001.

- Bevilacqua P., Rossi Doria M. (a cura di), Le bonifiche in Italia dal '700 ad oggi, Laterza, Roma - Bari 1984.

- Camagni R., Processi di utilizzazione e difesa dei suoli nelle fasce periurbane: dal conflitto alla cooperazione fra città e campagna, in BOSCACCI F., CAMAGNI R. (a cura di) «Tra città e campagna: periurbanizzazione e politiche territoriali», II Mulino, Bologna 1994, pp.13-85.

- CASATI D., Tendenze evolutive delle politiche agrarie, in FERRARESI G., Rossı A. «ll parco come cura e cultura del territorio: un percorso di ricerca sull'ipotesi del parco agricolo», Grafo, Milano 1993, pp.6072. 
- Charrier J.B., Geografia dei rapporti città - campagna, Franco Angeli, Milano 1994.

- CHILÒ L., La rivoluzione delle biotecnologie e la trasformazione del paesaggio agrario, Grafo, Brescia 1992.

- Commissione europea - Direzione generale dell'Agricoltura, Indicatori per l'integrazione della problematica ambientale nella Politica Agricola Comune, Bruxelles 2000.

- Commissione europea - Direzione generale dell'Agricoltura, La Politica Agricola Comune: sintesi 2000, Comunità europea, Lussemburgo 2001.

- Commissione europea - Direzione generale dell'Agricoltura, Riforma della PAC: Sviluppo rurale, Comunità europea, Lussemburgo 2001.

- CONTI L., Paesaggio agrario: storia consumata e storia possibile, in «Urbanistica Informazioni» nn.112-113, INU edizioni, Roma 1991.

- CONTI G., Abitare la distanza: dal radicamento rurale al teleantropismo metropolitano, in «Urbanistica Informazioni Dossier» n.9, INU edizioni, Roma 1997, pp.1-5.

- Corzani G. (a cura di), Alla ricerca dell'omogeneità perduta. II degrado delle zone agricole, in «Paesaggio Urbano» n.1, gen.-feb., Maggioli, Rimini 1996, pp.104-109.

- Di Rosa M., La salvaguardia del paesaggio rurale. Problemi, obiettivi e strumenti per una gestione "sostenibile", in BALDI M.E. (a cura di) «La riqualificazione del paesaggio», La Zisa, Palermo 1999, pp.305335.

- FabBri P., Natura e cultura del paesaggio agrario: indirizzi per la tutela e la progettazione, Città Studi, Milano 1997.

- Falasca C., Territori agricoli e pianificazione urbanistica, NIS, Roma 1983.

- Grosso N., Agricoltura, tutela dell'ambiente e conservazione del paesaggio: le frontiere dell'agro-ecologia nella CEE-UE, in «Rivista Geografica Italiana» n.103, annata CIII, giu., Pacini, Roma 1996, pp.215-225.

- Lucchetti W., harpes R., Laccone G. (a cura di), Storia della Politica Agricola Comune europea, pubblicazione a cura dell'Accademia Europea Agricoltura et Agroindustria, Verona 2002.

- Maniglio Calcagno A., Architettura del paesaggio. Evoluzione storica, Calderini, Bologna 1983. 
- Marocco M., Politiche e strumenti di intervento nelle aree agricole peri-urbane, in «Agricoltura Ambiente» n.8, giu., F.lli Palombi, Roma 1980, pp.30-56.

- Merlo M., Agricoltura, foreste e territorio. La formazione dei paesaggi rurali italiani e l'evoluzione nell'uso del territorio, in FABBRI M. (a cura di), «Pianificazione del territorio agricolo», INVET / Franco Angeli, Milano 1986, pp.13-39.

- Pirani A. (a cura di), Progetti della natura e dell'uomo, Franco Angeli, Milano 1999.

- Rizzo B.M., Trasformazioni dei modelli di paesaggio rurale e del rapporto città - campagna, in BILUCAGLIA T., GALASSI A., RICCI L., SANTANGELO S., VITTORINI A.M. "Percorsi di ricerca - Atti del $2^{\circ}$ convegno nazionale dei Dottorati di Ricerca in Pianificazione Territoriale e Urbanistica», Dedalo, Roma 1998, pp.128-131.

- Rossı DoRia M., La riforma sei anni dopo, in «Storia d'Italia», Einaudi, Torino 1976.

- Segrè A., Agricoltura e territorio nella politica agraria nazionale e comunitaria: tra difesa e valorizzazione delle risorse alimentari e ambientali, in "Paesaggio Urbano», suppl. ai nn.5-6, set.-dic., Maggioli, Rimini 2001, pp.21-27.

- Sereni E., Storia del paesaggio agrario italiano, Laterza, Roma Bari 1989.

- TURRI E., I/ paesaggio agrario, in TURRI E. «L'Italia vista dal cielo», Vallardi, Milano 1990, pp.153-180.

- Zagnoni S., La trasformazione del paesaggio agrario nella cartografia storica, in «Paesaggio Urbano» n.1, gen.-feb., Maggioli, Rimini 1995, pp.30-40.

\section{SUL NUOVO RUOLO DEL PAESAGGIO AGRARIO}

- AA.Vv., Carta di Napoli. Il parere degli specialisti sulla riforma degli ordinamenti di tutela del paesaggio in Italia, Napoli 1999.

- ANCI Toscana, Agricoltura e territorio: un laboratorio per lo sviluppo sostenibile della Toscana, Centro A-Zeta, Firenze 1996.

- BaRbanente A., Spazi rurali fra vecchie tendenze e nuovi ruoli, in «Urbanistica Informazioni» n.170, mar.-apr., INU edizioni, Roma 2000, p.27. 
- Bobbio R., A proposito di aree agricole: opportunità di un rinnovato interesse, in «Urbanistica Informazioni» n.152, mar.-apr., INU edizioni, Roma 1997, pp.24-25.

- BoRIANI M., Quale paesaggio per quale tutela?, in ScAzZosi L. (a cura di) «Politiche e culture del paesaggio: esperienze internazionali a confronto», Gangemi, Roma 1999, pp.13-16.

- CANNATA G., L'agricoltura e l'ambiente: tra conflitto e compatibilità, in Guido FABIANI (a cura di) «Letture territoriali dello sviluppo agricolo», Franco Angeli, Milano 1991, pp.203-224.

- CHILÒ L., La rivoluzione delle biotecnologie e la trasformazione del paesaggio agrario, Grafo, Brescia 1992.

- Di Rosa M., La salvaguardia del paesaggio rurale. Problemi, obiettivi e strumenti per una gestione "sostenibile", in BALDI M.E. (a cura di) «La riqualificazione del paesaggio», La Zisa, Palermo 1999, pp.305335.

- FAbBri P. (a cura di), Paesaggio, pianificazione, sostenibilità, Alinea, Firenze 2003.

- Grillotti di Giacomo M.G., Spazio funzionale e spazio politicoamministrativo nell'agricoltura italiana, in Bollettino della Società Geografia Italiana vol.XI n.1, gen.-mar., Società Geografia Italiana, Roma 1994.

- Kipar A., La produzione di valori ambientali nelle aree periurbane: verde agricolo, verde naturale, verde attrezzato, in Flavio BosCACCI, Roberto CAMAGNI (a cura di) «Tra città e campagna: periurbanizzazione e politiche territoriali», II Mulino, Bologna 1994, pp.395-406.

- LECHI F., Nuove funzioni per l'agricoltura periurbana e $i$ nuovi strumenti di intervento, in BOSCACCI F., CAMAGNI R. (a cura di) «Tra città e campagna: periurbanizzazione e politiche territoriali», II Mulino, Bologna 1994, pp.251-265.

- LEONE U., Il ruolo dell'agricoltura nella salvaguardia dell'ambiente, in SANTORO LEZZI C. (a cura di), «Ricostruire l'agricoltura per ricostruire l'ambiente», Congedo, Lecce 1983, pp.83-104.

- Marocco M., Politiche e strumenti di intervento nelle aree agricole peri-urbane, in «Agricoltura Ambiente» n.8, giu., F.lli Palombi, Roma 1980, pp.30-56.

- Monti C., Pratelli A., Riguzzi G., Secondini P., Analisi e pianificazione del territorio rurale, Clueb, Bologna 1985.

- Muraro G., La promozione di valori ambientali nelle aree periurbane non urbanizzate, in BOSCACCI F., CAMAGNI R. (a cura di) 
«Tra città e campagna: periurbanizzazione e politiche territoriali», II Mulino, Bologna 1994, pp.213-224.

- PANDAKOVIC D., Il paesaggio come identità: documenti e fonti per la lettura del paesaggio, in AA.VV., "Opinioni sul paesaggio», Ordine degli architetti della Provincia di Como, Como 2000, pp.17-21.

- PAOlella A., Il ruolo delle aree agricole nel progetto di ricostruzione della continuità, in «Attenzione. Rivista WWF per l'ambiente e il territorio», dossier 10/11, giu.-set., Roma 1998.

- Russu R. (a cura di), L'agricoltura e le pressioni sull'ambiente, ARSIA e Regione Toscana, Firenze 1997.

- Scazzosı L., Paesaggio, Paysage, Paisaje, Landscape, Landschaft, Landschap, Krajobraz... politiche e culture del paesaggio in Europa e negli Stati Uniti: una lettura trasversale, in ScAZzosı L. (a cura di) «Politiche e culture del paesaggio: esperienze internazionali a confronto», Gangemi, Roma 1999, pp.17-36.

- Schimdt O. e altri, Agricoltura biologica, Ed. agricole, Bologna 1994.

- Segrè A., Agricoltura e territorio nella politica agraria nazionale e comunitaria: tra difesa e valorizzazione delle risorse alimentari e ambientali, in «Paesaggio Urbano» suppl. ai nn.5-6, set.-dic., Maggioli, Rimini 2001, pp.21-27.

- Segre L., Evoluzione delle normative ambientali nella politica agraria comunitaria, in SEGRE L. (a cura di) «Agricoltura, ambiente e sviluppo economico nella storia europea», Franco Angeli, Milano 1993, pp.265-276.

- TORTORETO E., Strumenti normativi per la riqualificazione e il recupero delle aree periurbane, in TosI A. (a cura di) «Degrado ambientale periurbano e restauro naturalistico», Franco Angeli, Milano 1999, pp.123-142.

- Tosı A., Assetti agricoli e vegetazionali sostenibili contro il degrado e la diffusione insediativa periurbana, in Tosı A. (a cura di) «Degrado ambientale periurbano e restauro naturalistico», Franco Angeli, Milano 1999, pp.7-16.

- Tosı A., II contributo degli spazi rurali periurbani ad una pianificazione ambientale, in CHILÒ L. e TOSI A. (a cura di), "Cartografia e territorio rurale: l'uso dell'informazione cartografica in alcuni casi studio», Grafo edizioni, Brescia 1992, pp.7-15.

- Tosı A., Gli esiti di una ricerca sui paesaggi agrari, in «Territorio» n. 15, Franco Angeli, Milano 2000, pp.58-62.

- Von Meyer H., Considerazioni iniziali per un'analisi scientifica della conflittualità fra agricoltura ed ambiente e sulle implicazioni politiche 
relative, in SEGRE L. (a cura di) «Agricoltura, ambiente e sviluppo economico nella storia europea», Franco Angeli, Milano 1993, pp.255-264.

- ZAgNoni S., La trasformazione del paesaggio agrario nella cartografia storica, in «Paesaggio Urbano» n.1, gen.-feb., Maggioli, Rimini 1995, pp.30-40.

\section{SUGLI INDICATORI}

- Arpav, Rapporto sugli indicatori ambientali del Veneto - anno 2000, pubblicato sul sito www.arpa.veneto.it/via/report.htm, 2000.

- Colombo A.G., Malcevschi S. (a cura di), Manuale AAA degli indicatori per la Valutazione di Impatto Ambientale, vol. 5, Centro Via Italia, AAA e Fast, Milano giugno 1999.

- Commissione europea - Direzione generale dell'Agricoltura, Indicatori per l'integrazione della problematica ambientale nella Politica Agricola Comune, Bruxelles 2000.

- Cordara P., Indirizzi metodologici nell'analisi e nella valutazione della qualità visiva del paesaggio, in «Genio Rurale» n.7/8, 1994.

- FALINI P., I paesaggi rurali: storia e progettazione, in «Rassegna di architettura e urbanistica» nn.47-48, ago.-dic., Kappa, Roma 1980, pp.52-75.

- FAlini P., CIARDINI F., La qualità visiva del paesaggio: metodi e tecniche di valutazione, in «Agricoltura Ambiente» n.25, F.lli Palombi, Roma 1985, pp.23-35.

- FalinI P., BRUschi S., II paesaggio, in GISOTTI G., BRUSchI S. (a cura di), «Valutare l'ambiente», NIS, Roma 1990.

- GAmBI L., I valori storici dei quadri ambientali, in «Storia d'Italia: i caratteri originali», vol.1, Einaudi, Torino 1968, pp.5-60.

- GIUDICE M., Gli indicatori ambientali: strumenti per la pianificazione del territorio, in «Parchi» n.0, mag., Maggioli, Rimini 1990.

- LEONE U., Sugli indicatori di qualità ambientale, in MANZI E., SCHMIDT DI FRIEDBERG M. (a cura di), «Terra, ambienti, uomini: i geografi e gli indicatori ambientali», Marcos Y Marcos, Milano 1996.

- Malassis L., Passato e divenire dei paesaggi agricoli, in «Paesaggio mediterraneo», Electa, Milano 1992, pp.266-273. 
- Manzi E., Schmidt di Friedberg M. (a cura di), Terra, ambienti, uomini: i geografi e gli indicatori ambientali, Marcos $\mathrm{Y}$ Marcos, Milano 1996.

- Monti C., Pratelli A., Riguzzi G., Secondini P., Analisi e pianificazione del territorio rurale, Clueb, Bologna 1985.

- Pignatti S., Ecologia del paesaggio, Utet, Torino 1995.

- Schmidt di FriedBerg P. (a cura di), Gli indicatori ambientali: valori, metri e strumenti nello studio dell'impatto ambientale, Atti del convegno di Milano del 29-30 maggio 1984, Franco Angeli, Milano 1987.

- Vacca S., La valutazione dei caratteri del territorio nella pianificazione, metodi e applicazioni, Franco Angeli, Milano 1992.

- Venturelli Colantonio R., Contributo per la formulazione di un metodo di analisi della componente agricola dello spazio periurbano, in DELLA RocCA G.A., LAPADULA B.F. (a cura di), «Rapporti fra agricoltura e urbanistica nello spazio periurbano", atti di un «seminario» trasformato in una raccolta di studi in memoria di Valerio GIACOMINI, Cedam, Padova 1983, pp.191-210.

- ZerBi M.C., Futuro del paesaggio e paesaggio del futuro, in «VA Valutazione Ambientale» n.1, gen.-giu., Edicom, Milano 2002, pp.3642. 


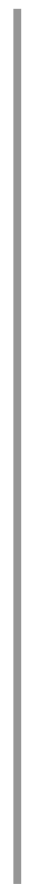

\section{PARTe SECONDA}

IL CAMPO APPLICATIVO: IL PAESAGGIO AGRARIO DELLA CAMPAGNA ROMANA 
La piana dell'Agro Romano si allargava a nord e a sud nella Maremma, una regione paludosa lungo la costa che raggiungeva da un lato il confine con la Toscana, e dall'altro Terracina, in prossimità della frontiera del vecchio Regno di Napoli. ${ }^{1}$

\section{DELIMITAZIONE E BREVE DESCRIZIONE DEL CAMPO APPLICATIVO}

Dal punto di vista "amministrativo", la campagna romana è stata intesa in diversi studi ${ }^{2}$ come quella zona compresa entro i vecchi confini del comune di Roma, che venne costituito nel 1848 seguendo il limite dell'antica "comarca", parola spagnola che ha come significati "circoscrizione, divisione territoriale, territorio".

II comune di Roma, peraltro, aveva nel 1871 e ancora nei decenni successivi una superficie maggiore di quella di 14 delle province italiane: data la moltiplicazione recente delle province, il comune, anche se ridotto in estensione, supera oggi un numero ancora maggiore di province, sia in estensione che in popolazione.

In senso orario il confine del territorio romano andava dalle porte dell'attuale Ladispoli (che prese il nome dal suo fondatore Ladislao ODESCALCHI nel 1888), a Cesano, ai pressi di Monterotondo, a Lunghezza in direzione di Tivoli, al margine dei colli Albani: a sud il confine del comune non corrispondeva più a quello dell'Agro Romano,

1 H. Gross, Roma nel Settecento, Laterza, Roma - Bari 1990, p.176.

2 Uno dei più importanti studi che si è basato su questo tipo di delimitazione è quello condotto dal prof. Lando BORTOLOTTI, che ha portato alla stesura del volume Roma fuori le mura edito da Laterza nel 1988. 
includendo parte dell'Agro Pontino, con Ardea e una striscia di territorio che si spingeva a meno di cinque chilometri dal lago di Fogliano.

La maggior parte delle ricerche che fino ad oggi si sono occupate dell'ambito territoriale della campagna romana si sono solitamente riferite a confini di carattere "strutturale",

alludendo come campagna romana allinsieme del territorio - intorno a Roma - che è delimitato da una parte dai crinali dei monti che circondano Roma e dall'altra parte dalla costa, e che costituisce il bacino idrografico di Roma. $^{3}$

Dal punto di vista della struttura fisica della campagna romana, una prima delimitazione del campo applicativo può essere quindi fatta seguendo la conformazione assunta dal sistema idrografico.

La ricchezza d'acqua è stata, sin dalle origini della città e durante tutta la sua evoluzione, una delle grandi risorse di Roma, innanzitutto per la presenza dei due fiumi: il Tevere ed il suo principale affluente, l'Aniene. Oltre a questi altra ricchezza d'acqua deriva dalla moltitudine di sorgenti di varia tipologia presenti nel territorio, entro e fuori le mura; dal fitto reticolo di vene d'acqua che scendono verso la piana dove si è sviluppata la città; dalla corona dei monti che la circondano (in senso antiorario: i Colli Albani, i monti Tiburtini, i Lucretili, i Cornicolani e i Sabatini); dalle grandi riserve d'acqua della media valle dell'Aniene, da cui partivano i principali acquedotti romani; dalle riserve dei laghi di Albano a sud e di Bracciano a nord.

La campagna romana potrebbe essere, dunque, divisa in due settori a seconda dell'andamento assunto dalla rete idrografica minore appartenente al bacino del fiume Tevere.

3 V. CAlzolaRI, Ambito e ambiti della campagna romana come letture e prospettive per la pianificazione, intervento al seminario «Strumenti e metodi per la conoscenza della campagna romana», Dottorato di ricerca in Progettazione Paesistica, Roma 2000. 
Nel settore nord orientale, sia i fossi che le marrane che scendono dai complessi vulcanici dei monti Sabatini e dei Colli Albani delineano un andamento centripeto rispetto al centro della città. Da questa configurazione resta escluso il corso del fiume Aniene che, con il suo andamento tangente rispetto all'insediamento urbano, rappresenta il secondo fiume di Roma. Dal 1924 circa il fiume è stato inglobato nella costruzione urbana con i quartieri di Monte Sacro prima, Ponte Mammolo e S.Basilio successivamente.

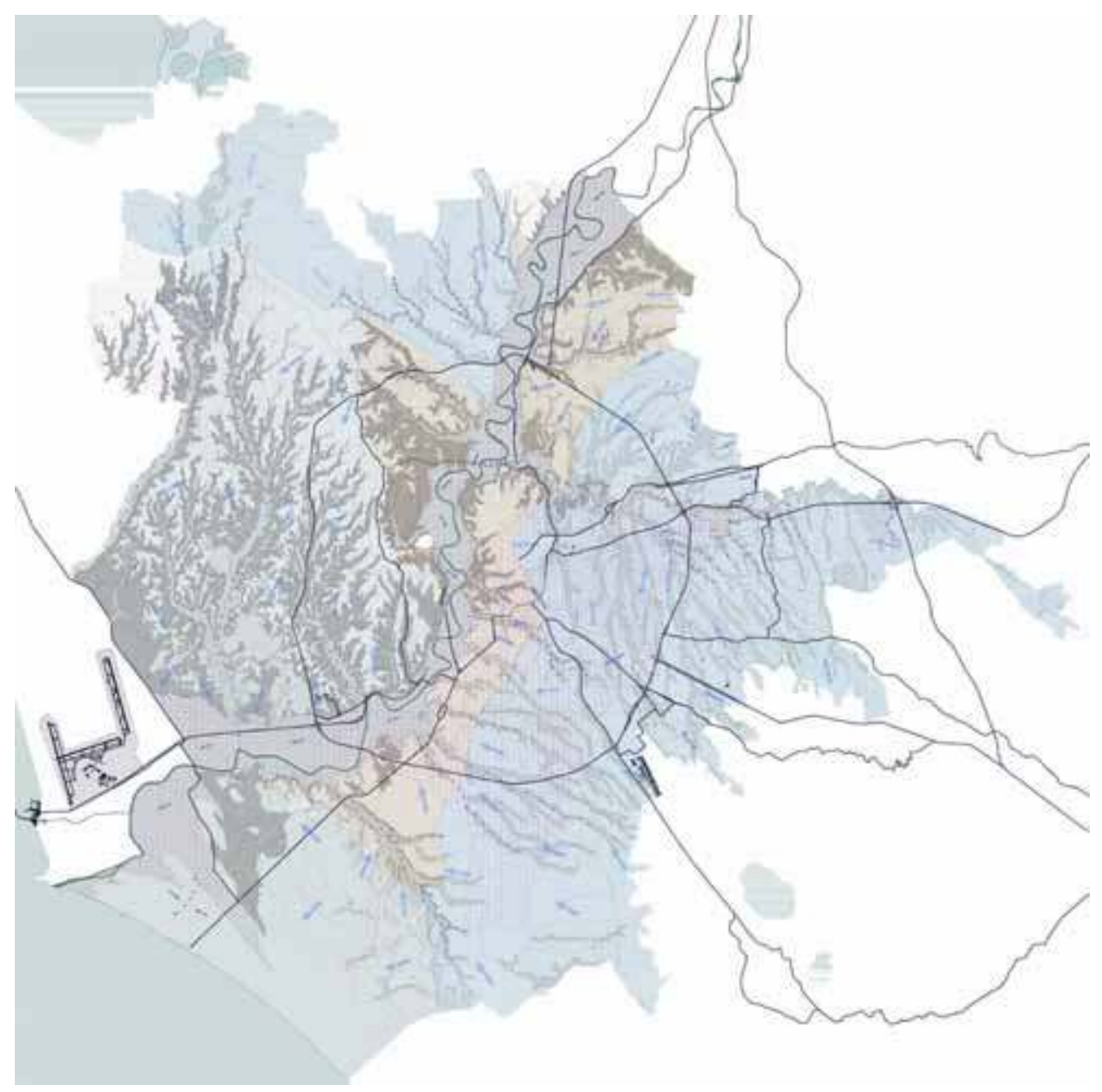

Figura 1. Idrogeologia del territorio comunale.

Al contrario, nel settore sud occidentale le direttrici idriche tributarie del Tevere hanno un andamento tangente il perimetro urbano. II più lungo di questi affluenti, che è anche l'ultimo in quanto sbocca nel 
Tevere immediatamente prima della sua foce a delta nel mare, è il fosso Galeria che con le sue acque perenni è caratterizzato da una valle relativamente ampia. Proseguendo verso ovest il territorio è poi interessato dalla presenza del fosso Arrone che, nascendo dal complesso vulcanico sabatino, ha un bacino imbrifero indipendente da quello del Tevere e infatti sfocia direttamente a mare all'altezza dell'insediamento di Fregene.

Dal punto di vista geo-morfologico la campagna romana è interessata innanzitutto da una vasta area pianeggiante che occupa in larga parte la parte centrale e terminale del territorio, comprendendo la valle del Tevere e l'ampia zona costiera bonificata, testimone quest'ultima del continuo avanzamento del delta del fiume nelle diverse epoche geologiche e storiche.

II resto del territorio, formato da una serie di ripiani tufacei più o meno incisi, è caratterizzato dalla presenza di aree con conformazioni morfologiche e geologiche un po' diverse.

Una prima zona è quella compresa tra la via Laurentina e l'Aniene, interessata dalle ultime pendici poco inclinate dell'apparato vulcanico dei colli Albani, formate da tufi intercalati o ricoperti da uno strato più 0 meno spesso di "pozzolana incoerente". A queste propaggini segue lungo la via Appia, procedendo in senso antiorario, uno strato di colata lavica che è stato inciso da brevi corsi d'acqua che non hanno dato origine a grandi incisioni vallive. II risultato è quello di una vasta distesa quasi del tutto pianeggiante, solcata da fossi non molto profondi, che non hanno disturbato le coltivazioni agricole.

Continuando nello stesso senso di marcia questa descrizione, a parte il fiume Aniene che scorre in una valle relativamente ampia, quasi tutto il restante territorio a sud e ad ovest è caratterizzato da una morfologia più movimentata e complessa. Oltre la via Nomentana, quindi, 
ove si incontrano l'ormai poco spessa coltre del Vulcano Laziale e quella dell'altrettanto lontano complesso vulcanico Sabatino, più lunghi corsi d'acqua hanno inciso valli più ampie con pendii più larghi. ${ }^{4}$

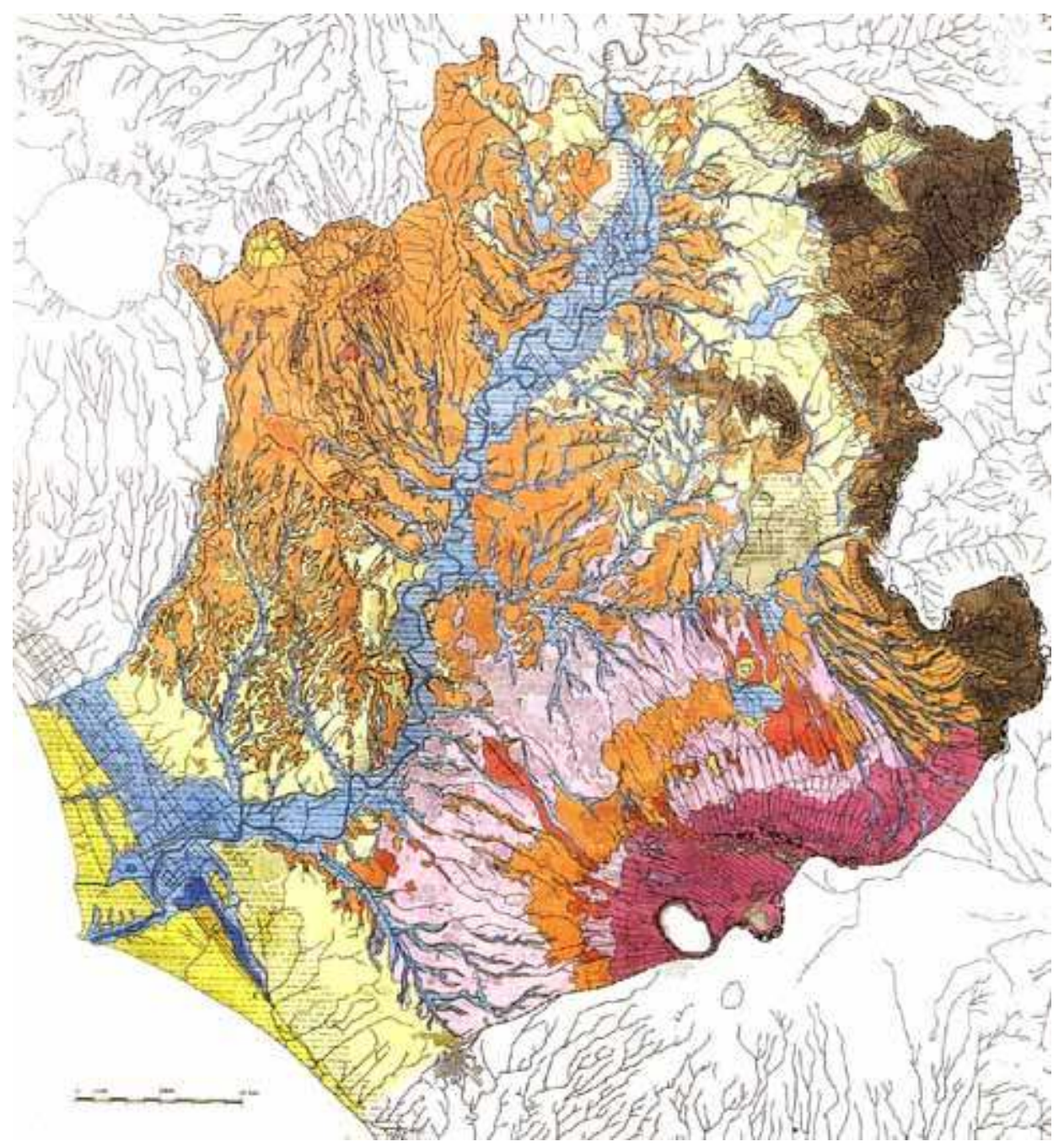

Figura 2. La carta litomorfologica del bacino idrografico di Roma

Questa conformazione particolare è dovuta principalmente al fatto che è stata raggiunta la sottostante coltre sedimentaria clastica, ovvero una formazione geologica più soggetta alla formazione di una morfologia più arrotondata. Fanno parte, ad esempio, di questa

4 E. PARATORE, I/ suburbio geo-agrario di Roma, Istituto di Studi Romani, Roma 1979, p.36. 
porzione della campagna romana la valle percorsa dalla via Palombarese (solcata da un fosso che assume diversi nomi nei suoi diversi tratti), oppure quella percorsa dal fosso della Magliana, o quella del fosso Galeria, o ancora quella del fosso Arrone.

In questa zona molte parti scoscese - soprattutto quelle lungo i fianchi ripidi che costeggiano le valli più strette - si sottraggono a qualunque tipo di coltivazione e sono solitamente ricoperte da una boscaglia del tipo macchia mediterranea, che a volte è talmente rada e bassa da permettere il pascolo.

Infine, man mano che ci si avvicina alle pendici del Vulcano Sabatino, la zona nord occidentale del territorio presenta una morfologia più simile a quella descritta per l'area orientale.

In un territorio così articolato morfologicamente non poteva mancare un altrettanto varia qualità dei terreni, tanto che dal punto di vista pedologico è possibile riconoscere sei areali caratteristici.

La prima zona comprende la valle del Tevere e la zona mediana del suo delta ed è costituita da terreni alluvionali mediamente umiferi, freschi e di buona e media fertilità. Questa porzione di territorio, compresa tra il cordone di dune più antico e quello di più recente formazione, è il suolo acquitrinoso interessato dalla bonifica idraulica di Ostia e Maccarese: si tratta di una categoria di terreni particolarmente favorevole a qualsiasi tipo di coltivazione, anche vista la regolarità geometrica degli appezzamenti.

Vi è poi la zona costiera vera e propria, profonda circa $4 \mathrm{~km}$ all'altezza di Ostia e Fiumicino e che si riduce solamente a qualche decina di metri verso il confine comunale sempre lungo la costa. È un'area occupata da sabbie litoranee delle dune recenti, povere di humus, aride e con una fertilità abbastanza bassa. In compenso questa zona, essendo stata a suo tempo spianata e canalizzata dalle opere 
della bonifica idraulica, è facilmente accessibile per il lavoro meccanico e l'irrigazione.

Con caratteristiche pedologiche simili e caratterizzati da discreta o bassa fertilità sono i terreni interessati dalle dune pleistoceniche, che si trovano distribuite lungo la fascia che occupa la fronte iniziale dell'antico delta con un andamento nord ovest - sud est. A differenza di quelli precedenti, questi terreni, pur irrigabili, non sono completamente pianeggianti e sono stati interessati solo marginalmente dalle opere di bonifica idraulica dell'inizio del ' 900 .

Una quarta zona è quella compresa tra il fosso della Crescenza e S. Maria di Galeria a nord e tra la valle del Tevere e la duna quaternaria a sud. È interessata da terreni prevalentemente argillosi e sabbiosi, che nella parte sommitale sono ricoperti da una strato vulcanico tufaceo denominato «cappellaccio» ${ }^{5}$. Questi terreni, ben provvisti di humus e di media fertilità, sono però molto aridi e necessitano quindi di consistenti opere di irrigazione che, talvolta,

sono state attuate a costo di sacrifici finanziari non sempre corrisposti dalla convenienza economica. ${ }^{6}$

Oltre questa zona, ruotando in senso antiorario intorno alla città sino a ritrovare le dune quaternarie della sponda sinistra del Tevere, vi è l'area a ridosso delle pendici del Vulcano Laziale, nella quale i terreni affioranti sono esclusivamente composti da terreni vulcanico - tufacei, discretamente umiferi, di media fertilità, ma alquanto aridi. A questi si aggiungono una "lingua" lavica lungo la via Appia Antica, due ad est del fosso dell'Osa, a direzione nord ovest - sud est, ed un'altra nella zona della Casaccia, a destra del fosso Arrone lungo il confine comunale,

5 In questa sorta di crosta, che spesso si presenta dura e poco permeabile, è stata attuata una vera e propria opera di scasso (profonda anche più di un metro) in maniera tale da permettere la coltivazione ed un certo assorbimento delle acque.

6

E. PARATORE, op. cit., p.39. 
che danno origine a terreni rocciosi particolarmente aridi, ma con una discreta fertilità.

Un'ultima classe di terreni si sviluppa tanto nella zona vulcanicoclastica, quanto in quella esclusivamente vulcanica: qui una fitta ramificazione di fossi principali ha determinato la formazione di fondovalli interessati da terreni alluvionali prevalentemente vulcanici ben provvisti di humus, freschi e con un'elevata fertilità. Queste venature sono facilmente irrigabili e pianeggianti, ma non sono mai molto ampie e, essendo caratterizzate da fossi e valli ad andamento particolarmente tortuoso, presentano appezzamenti di terreni che difficilmente si possono squadrare e quindi sono di difficile coltivazione.

II nostro campo applicativo - uniformandosi a una serie di studi già condotti sullo stesso territorio ${ }^{7}$ - corrisponde, dunque, al territorio del bacino orografico dell'area romana, delimitato a nord-ovest dal distretto vulcanico dei monti Sabatini e dalle loro propaggini verso la valle del Tevere e a sud-est dal distretto vulcanico dei Colli Albani. La delimitazione a nord è poi costituita dalla corona delle formazioni montuose di tipo calcareo dei monti Prenestini, Tiburtini e Lucretili. A sud, infine, il limite è rappresentato dalla linea costiera, tra la foce del fosso Arrone e la macchia della tenuta di Castel Porziano.

L'area di studio, quindi, coincide con il basso bacino del Tevere, fino alla confluenza del fiume Farfa, con l'esclusione del bacino dell'Aniene a monte di Tivoli. Vengono invece compresi nell'area il bacino del fosso Arrone e la fascia costiera, in quanto sono strettamente connessi sotto il profilo territoriale.

$7 \quad$ Ci si riferisce soprattutto agli innumerevoli studi che sulla campagna romana sono stati condotti dal gruppo di studio coordinato dalla prof.ssa Vittoria Calzolari e che nel periodo più recente ha portato alla realizzazione del volume: V. CALzoLARI, Storia e natura come sistema: un progetto per il territorio libero dell'area romana, Àrgos, Roma 1999. 


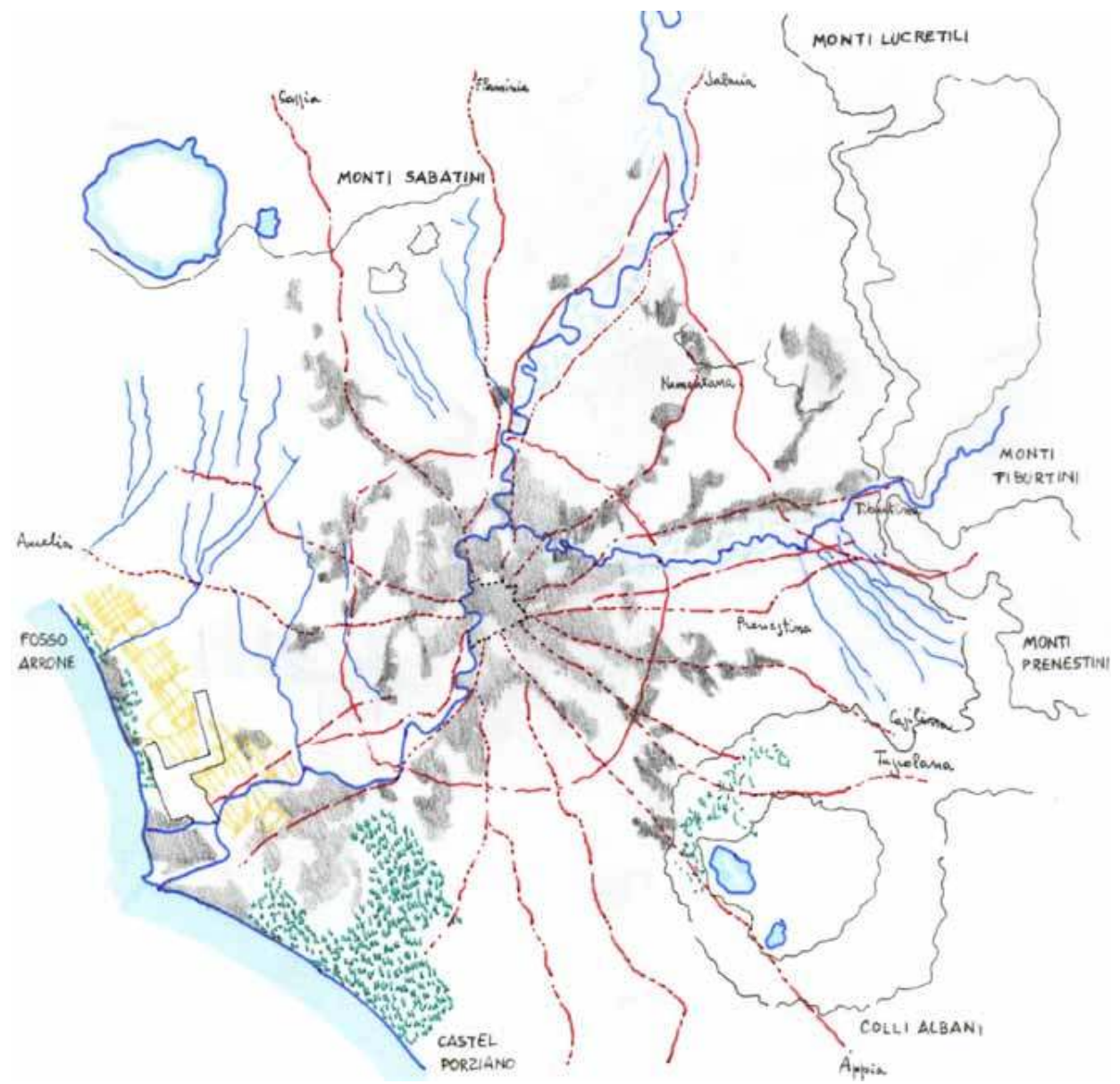

Figura 3. II campo applicativo. 
Più uniforme, più disalberato l'Agro Romano, che era solo raramente punteggiato di casali spesso con aspetto di fortilizi, e con funzione di fattorie di tenute vastissime. Ma appunto perché più spoglio, i pini a ombrello solitari o i ciuffi di eucalipti (piantati a partire dal secolo scorso) ne costituiscono un ornamento di maggior risalto, insieme alle suggestive arcate degli antichi acquedotti, alle rovine di ville e monumenti sepolcrali romani. Altri elementi del paesaggio tradizionale, ritratto in romantiche vecchie stampe, sono invece quasi scomparsi: così le alte capanne coniche dei pastori o le mandrie di bufali e di cavalli. ${ }^{1}$

\section{CAUSE ED EFFETTI DELLE PRINCIPALI TRASFORMAZIONI NEL PAESAGGIO DELLA CAMPAGNA ROMANA}

Per un lungo periodo e fino almeno al XIX secolo Roma era l'unica grande città europea circondata da campagne malariche $e$ da paludi prive di abitanti, senza campi coltivati, senza alberature. Una città popolosa in mezzo a un deserto, volutamente ignorato dai romani.

Se lo si va a rivedere, il territorio intorno alla città - per un periodo che va dal 1300 circa al 1870 - è stato pressoché abbandonato, così come riferiscono tutti $i$ viaggiatori che descrivono il paesaggio romano. Un paesaggio desolato, con le

\footnotetext{
A. SestinI, II Paesaggio, in "Conosci l'Italia», vol.IV, Touring Club Italiano, Milano 1963, pp.133-135.
} 
grandi rovine che sollecitavano l'interesse e la fantasia, con un forte contrasto tra il territorio abbandonato, da un punto di vista paesistico-ambientale, e il valore simbolico che acquistava in quei periodi.

L'agro romano, paesaggio di colline e latifondi quasi incolti e talora paludosi, solcati da arcigni torrenti e segnati dal cammino stagionale delle greggi, aveva già impressionato Montagne tre secoli prima, ma stupiva ancora $i$ pellegrini dell'anno Santo 1900 o i turisti dell'Esposizione internazionale del 1911. L'Europa non conosceva nessun'altra grande città che si collocasse così clamorosamente nel bel mezzo di un territorio spopolato. ${ }^{2}$

Com'era realmente l'Agro al momento dell'Unità d'Italia?

Al tempo della fine del potere temporale dei papi, la città costruita occupava solo parte dell'area racchiusa dalla cerchia delle mura Aureliane, poco più del 40\%; le costruzioni si alternavano con ville, giardini, vigne e orti. Al di là delle mura il suburbio formava intorno alla città una fascia larga tra i 2 e i $6 \mathrm{~km}$; oltre questa fascia si estendeva l'Agro romano, che era coltivato in piccola parte: il $90 \%$, infatti, era dedicato al pascolo delle greggi che venivano dalle regioni montane intono a Roma, mentre la parte a coltura era gestita "a quarteria", cioè coltivata per un anno e lasciata a riposo per tre.

Le coltivazioni erano di tipo estensivo: non vi erano sistemazioni agrarie e alberature, nessun servizio per i coltivatori avventizi che scendevano dai colli e dai monti nelle stagioni dei lavori agricoli, rarissime strade in pessimo stato. II rapporto di Roma col suo territorio si riduceva alla funzione di magazzino che

2 A. Caracciolo, F. QuILICI, Roma, una capitale singolare, il Mulino, Bologna 1985, p.65. 
la città svolgeva per l'Agro, rendendola qualcosa di simile a una gigantesca azienda silvo-pastorale. $^{3}$

Come afferma Lando BORTOLOTTA', anche se il rapporto fra la città e la sua campagna era del tutto anomalo, è inevitabile accennare - nel sintetizzare le vicende dell'Agro - a quelle parallele della città.

$\dot{E}$ bene ripercorrere, in maniera sintetica, alcune delle tappe principali della storia di questa porzione di territorio e della città, attraverso le quali leggere le trasformazioni strutturali più evidenti che hanno dato origine all'attuale conformazione del territorio della campagna romana:

- l'evoluzione del regime fondiario, attraverso l'analisi dei cambiamenti subiti dalle proprietà terriere e della modifica del concetto stesso della rendita;

- le opere della bonifica idraulica ed agraria che hanno interessato la zona costiera, modificandone radicalmente i caratteri originari;

- l'infrastrutturazione che ha interessato questo territorio, fungendo alcune volte da elemento base per una valorizzazione della campagna romana, altre volte - nei periodi più recenti rappresentando un nuovo elemento di "disordine";

3 In una descrizione della campagna romana presentata dal Ministero dell'Agricoltura, Industria e Commercio in occasione dell'Esposizione Universale di Parigi del $1878 \mathrm{si}$ legge:

Si apre una regione pianeggiante e largamente ondulata che va a perdersi verso sud-ovest nel mare Tirreno. Questa planizie di superficie ineguale, incisa dal Tevere e da' suoi affluenti che vi scorrono incassati entro profondi solchi, presentasi quasi ovunque incolta e a soli pascoli naturali, nuda d'alberi e di case, sede di mal'aria in estate. Nel mezzo di simile regione sparsa di rovine d'acquedotti e non priva invero di un certo carattere di severa grandiosità, sorge quasi d'improvviso la città, inclusa nel circuito di un antico muro che in gran parte è ancora quello di Adriano, e soltanto una zona di poca ampiezza di terreni tenuti a vigneto intramezzato da qualche grandiosa villa, forma di transito fra il deserto e l'abitato.

L. BoRtolotTI, Le persistenze della Campagna Romana: alcuni aspetti della sua evoluzione storica, intervento al seminario «Strumenti e metodi per la conoscenza della campagna romana», Dottorato di ricerca in Progettazione Paesistica, Roma 2000. 
- l'urbanizzazione che, il più delle volte in maniera incontrollata, ha via via impegnato aree sempre più ampie e consistenti della campagna romana. 


\section{LA PROPRIETÀ E LA RENDITA: EVOLUZIONE DEL REGIME FONDIARIO}

Il paesaggio agrario dell'area romana negli anni dell'unificazione si presentava come una delle zone più arretrate della penisola, ove il latifondo rappresentava la caratteristica strutturante, le tecniche e i metodi di coltivazione utilizzati erano particolarmente obsoleti e i nobili e i religiosi erano gli esclusivi proprietari dei terreni agricoli.

Alla fine del Settecento

dal punto di vista del possesso della terra, l'Agro Romano conteneva la massima concentrazione di proprietari terrieri privilegiati in tutta la penisola. Le 111.794 rubbia di terra $(206.818,9 \mathrm{ha})$ erano suddivise in 357 tenute. Di queste, 170 , pari a circa il $54,6 \%$ della terra, appartenevano a 113 famiglie nobili; e altre 153 , ossia il $42,8 \%$, erano nelle mani di istituzioni ecclesiastiche. ${ }^{5}$

Questo processo di concentrazione della proprietà della terra in poche mani non mutò nel secolo successivo, tanto che ancora

nel 1870 i circa 200.000 ettari del territorio comunale di Roma risultavano divisi in 388 poderi accentrati nelle mani di soli 204 proprietari, con una media di circa 1.000 ettari a proprietario già molto elevata se confrontata con quella di altre regioni italiane nella stessa epoca. ${ }^{6}$

La concentrazione latifondistica della proprietà dell'Agro veniva poi confermata dal fatto che soltanto tre proprietari possedevano insieme più di un quarto delle aree coltivate: il principe Borghese, con 22.149 ettari, il Capitolo di San Pietro, con 20.162 ettari, l'Ospedale di Santo Spirito, con 15.130 ettari.

Nello specifico, le proprietà dell'Agro si possono distinguere secondo le quantità di seguito riportate ${ }^{7}$ :

5 H. Gross, Roma nel Settecento, Laterza, Roma - Bari 1990, p.181.

O. MANCINI, I/ territorio agricolo dell'area metropolitana di Roma, in P. BoLCHINI, D. LORANDI (a cura di), «Metropoli e agricoltura», Franco Angeli, Milano 1982, p.265.

7 I dati, riferiti alla situazione in essere intorno al 1803, sono estrapolati da H. Gross, op. cit., p.184. 
Proprietà nobiliari

\begin{tabular}{|l|l|l|l|l|l|}
\hline \multicolumn{1}{|c|}{ Proprietari } & $\%$ area & \multicolumn{1}{c|}{ Proprietari } & $\begin{array}{c}\% \\
\text { area }\end{array}$ & \multicolumn{1}{c|}{ Proprietari } & $\begin{array}{c}\% \\
\text { area }\end{array}$ \\
\hline Borghese & 10,67 & Albani & 1,44 & Lepri & 0,97 \\
\hline Sforza Cesarini & 4,19 & Di Pietro - Giraud & 1,4 & Serluppi & 0,93 \\
\hline Patrizi & 2,8 & Salviati & 1,32 & Santacroce & 0,887 \\
\hline Chigi & 2,42 & Duca di Bracciano & 1,24 & Strozzi & 0,88 \\
\hline Altieri & 2,26 & Riccardi & 1,22 & Mattei & 0,84 \\
\hline Ruspoli - Falconieri & 1,88 & Barberini & 1,2 & Giustiniani & 0,78 \\
\hline Doria Pamphili & 1,74 & Torregiani & 1,15 & Lante & 0,63 \\
\hline Rospigliosi & 1,73 & Carpegna & 0,997 & Caffarelli & 0,56 \\
\hline
\end{tabular}

Proprietà ecclesiastiche

\begin{tabular}{|c|l|l|c|l|l|}
\hline Proprietari & $\%$ area & \multicolumn{1}{|c|}{ Proprietari } & $\begin{array}{c}\% \\
\text { area }\end{array}$ & \multicolumn{1}{|c|}{ Proprietari } & $\begin{array}{c}\% \\
\text { area }\end{array}$ \\
\hline Capitolo di S. Pietro & 12,4 & $\begin{array}{l}\text { Capitolo di S. } \\
\text { Giovanni in } \\
\text { Laterano }\end{array}$ & 2,19 & $\begin{array}{l}\text { Collegio } \\
\text { Germanico }\end{array}$ & 1,18 \\
\hline Ospedale S. Spirito & 7,47 & $\begin{array}{l}\text { Capitolo di S. Maria } \\
\text { Maggiore }\end{array}$ & 1,36 & $\begin{array}{l}\text { Capitolo di S. } \\
\text { Lorenzo in } \\
\text { Damaso }\end{array}$ & 0,36 \\
\hline
\end{tabular}

La grande proprietà che dominava l'area romana aveva puntato tutto sul grano e sul pascolo: il primo perché richiedeva uomini soltanto nei mesi della mietitura e della trebbiatura; il secondo perché rappresentava soltanto un momento, nel periodo autunnale ed invernale, di un sistema di transumanza e spostamento delle greggi e degli uomini verso la montagna abruzzese.

In realtà si trattava di un sistema complesso: dagli Appennini gli uomini scendevano a valle per lavorare la terra e la pianura, con le sue grandi proprietà nobiliari, aveva bisogno della montagna, non potendo fare a meno delle risorse umane e produttive che lì risiedevano.

Le colture che venivano attuate erano - come si è detto - quelle tradizionali della coltivazione del grano e di pochi altri cereali, effettuate con il "sistema della quarteria". Erano quasi completamente assenti l'olivo e la vite.

I proprietari di questi terreni, sia i nobili che il clero, non intervenivano mai direttamente nella produzione agricola, che veniva 
invece affidata ai fittavoli, con affitti che venivano quasi sempre rinnovati. La nobiltà romana, infatti, non aveva alcuna inclinazione per la vita rurale e, anche se si trasferiva in campagna per qualche mese durante i periodi più salubri della primavera e dell'autunno, la famiglia aristocratica si portava in realtà dietro tutte le comodità e le abitudini della vita di città.

II risultato era che quasi il $90 \%$ delle proprietà era ceduto in affitto con contratti che di media duravano tra i nove e i dodici anni (per le terre di proprietà ecclesiastica potevano scendere anche fino a tre anni). In molte occasioni, poi, gli stessi fittavoli a loro volta subaffittavano i terreni a pastori e contadini, a prezzi molto più alti.

Un affitto di questa durata non era in realtà lungo abbastanza da incoraggiare il fittavolo ad apportare migliorie permanenti al fondo, tanto più che neanche lui era radicato alla terra ${ }^{8} \mathrm{e}$, impegnato com'era ad applicare una cultura di rapina, poco o nulla concedeva alle migliorie. Le manutenzioni da effettuare sui territori presi in affitto, infatti, in questo quadro di relazioni tra proprietà ed imprese diventavano solo un aggravio delle spese di produzione che non si rifletteva in maniera altrettanto consistente sull'incremento delle rendite agricole.

Pian piano, però, ebbe luogo un processo che vide la concentrazione degli affitti nelle mani di un numero di fittavoli sempre più piccolo: erano i cosiddetti «mercanti di campagna», che già alla fine del '700 costituivano uno dei principali pilastri dell'economia dell'Agro.

La storiografia non è sempre stata unanime del valutare il ruolo svolto dal mercante di campagna entro il sistema agrario del Lazio, e più specificatamente dell'Agro. V'è chi l'ha visto come un elemento puramente parassitario, e chi l'ha considerato l'unica forza dinamica e intraprendente in seno ad un'economia altrimenti statica. $^{9}$

$8 \quad$ Vi sono molti casi in cui i terreni assunti da singoli fittavoli assumevano dimensioni ancora più estese dei latifondi dei nobili e dei preti.

9

H. GRoss, op. cit., p.187. 
Sicuramente i proprietari preferivano affidare le loro terre a questi mercanti piuttosto che ad una folla di coltivatori, soprattutto perché in questo modo avevano minori problemi amministrativi ed erano certi di essere pagati regolarmente. Inoltre, nella maggior parte dei casi, il fittavolo offriva al proprietario un reddito che era molto vicino a quanto quest'ultimo avrebbe potuto ottenere se avesse gestito in prima persona i suoi terreni. II «mercante di campagna», dal canto suo, prendeva in affitto una o più tenute da un unico proprietario (o in alcuni casi da più proprietari), e in parte le coltivava direttamente, in parte le subaffittava.

Un intellettuale di levatura europea come De Sismondi, intorno al 1860, descriveva la campagna romana dicendo

che si poteva attraversare i vasti latifondi romani per decine di miglia senza incontrare alcun segno di antropizzazione stabile del territorio. Esso si estende come un deserto punteggiato da grano, da macchie e boschi cedui senza alcuna traccia umana. Rari erano i casolari organizzati ad ospitare l'azienda agricola pastorale tipica del latifondo romano. ${ }^{10}$

Di questa presenza umana rarefatta risentiva l'intera maglia di centri rurali che costellavano, soprattutto verso le parti più in rilievo, la campagna romana. Pochissimi centri - Anguillara, Bracciano, Trevignano, Civita Castellana, Monterotondo, Larino, Albano, Marino superavano il migliaio di abitanti; altri centri, poi, si riducevano ad agglomerati di capanne e case con pochissimi abitanti.

La rarefazione demografica, le coltivazioni estensive ed un mercato del lavoro fondato sulle imponenti migrazioni stagionali, in sostanza, segnavano la struttura dell'azienda agraria e dell'agricoltura della campagna romana.

I flussi migranti di manodopera che si muovevano dalla montagne verso valle si indirizzavano verso le poche strutture insediative presenti,

10 S. DE SISMONDI, Del metodo di restaurare l'agricoltura nella campagna romana, s.e., s.d., p.152. 
che assumevano il carattere di vero e proprio avamposto produttivo nella campagna disabitata, perché oltre queste si apriva una nettissima divaricazione fra campi e case.

Questo senso di precarietà del rapporto tra i lavoratori e la terra si inseriva nel rapporto coerente e razionale di utilizzazione delle migrazioni interne che si andava strutturando in Italia nel XIX secolo. Non si trattava, infatti, di un movimento disordinato che si riversasse nella pianure alla ricerca disperata di un impiego: il mercato del lavoro era organizzato sulla base di una coerenza interna molto forte. Le grandi aziende cerealicole avevano, infatti, lavoratori dipendenti stabili e avventizi che, a seconda del ruolo che rivestivano, potevano ottenere un contratto di lavoro annuale, mensile o a cottimo.

In un'organizzazione del lavoro siffatta diventava elemento essenziale la mediazione:

era, infatti, impensabile che il massaro, il mercante di campagna 0 il faccendiere potessero stipulare singoli contratti di lavoro per le centinaia di donne e di uomini necessari alle complesse e lunghe operazioni agricole. Ciò diventava ancora più difficile perché il mercato del lavoro non esisteva sul posto. $^{11}$

II "caporale" romano aveva punti di riferimento nei diversi paesi dell'Appennino dai quali proveniva la manodopera e in quei paesi si recava a reclutare i braccianti, per avviarli poi verso le grandi aziende cerealicole, dove avrebbero formato il nucleo principale degli "annaroli”.

Accanto a queste forme migratorie, che si potrebbero definire «organizzate», vi erano altri flussi «indipendenti», come ad esempio i contadini che si organizzavano in compagnie autonome e, dai propri paesi di origine, scendevano verso le pianure, certi di trovare un lavoro stagionale, più breve ma anche molto più remunerativo.

11 G. ORLANDO, op. cit., p.122. 
Legata ad una logica produttiva che, come detto, era caratterizzata dall'uso estensivo del territorio e da un'organizzazione del lavoro precisa e strutturata, l'azienda agraria si fondava su un'organizzazione degli spazi, del lavoro e delle dimore rurali del tutto particolare: il casale non rappresentava, infatti, la dimora stabile di una o più famiglie contadine, ma era il centro di un organismo aziendale basato sul lavoro dei salariati (annuali, mensili o anche giornalieri), che avevano nel centro urbano la loro residenza principale ${ }^{12}$. La desertificazione del paesaggio della campagna romana e la scarsità, al suo interno, di insediamenti umani, dipende proprio dall'utilizzazione funzionale dei centri aziendali.

I casali romani che ai censimenti tardo-ottocenteschi risultavano abbandonati $(. .$.$) , in realtà erano pienamente utilizzati nei periodi agricoli$ collegati alle colture, per poi spopolarsi immediatamente dopo la mietitura ${ }^{13}$.

II casale doveva essere una struttura di servizio alla produzione, organizzata per rispondere al precario rapporto tra uomo e agricoltura che esisteva in pianura a quei tempi e in quelle zone. Questa struttura di servizio, in alcuni casi anche di grande dimensione e con qualche pretesa architettonica ${ }^{14}$, si organizzava su diversi piani di utilizzazione: i magazzini per il grano, i locali per il ricovero del fattore, la panetteria, qualche volta la cappella di campagna, i depositi e i piccoli locali dove si lavorava il formaggio, ecc...

L'assetto fondiario tipico del XVIII-XIX secolo iniziò a modificarsi con l'unificazione dello Stato Italiano e l'investitura di Roma a capitale. Mentre i secoli precedenti erano stati caratterizzati da una politica immobilistica dello Stato della Chiesa, il nuovo governo dimostrò una

12 Cfr. S. Russo, Una dimora per leggere il paesaggio, in «Viaggi di Erodoto», II, n. 5 , sett., Roma 1988, pp.98-107.

13 G. ORLANDO, Le campagne: agro e latifondo, montagna e palude, s.e., s.d.

14 Sulle diverse tipologie architettoniche dei casali nella campagna romana si può consultare C. TAGLIAFERRI, I casali della campagna romana, Pieraldo, Roma 1991. 
certa dinamicità e si uniformò ai nuovi interessi economici che stavano nascendo in quel periodo, dando origine alla formazione della rendita fondiaria.

In quel periodo venne promulgata una serie di leggi sull'abolizione dell'asse ecclesiastico ${ }^{15}$, che avrebbero potuto rappresentare una sorta di premessa per una riforma agraria, ma che al contrario si dimostrarono solo il preludio del successivo sviluppo urbano. A seguito di tali leggi il clero vendette, infatti, i propri beni: alla compravendita parteciparono i ricchi borghesi, i nobili e le grandi banche italiane e straniere, ma non il Comune e lo Stato, che, in questo modo, persero un'eccellente occasione per formare un demanio comunale che fungesse, poi, da elemento di controllo dello sviluppo urbano e del relativo mercato delle aree che da lì a poco si sarebbe scatenato.

In realtà, tanto a livello finanziario, che a livello economico e sociale, la liquidazione dell'asse ecclesiastico fu, nella campagna romana, tutt'altro che un successo, ad eccezione del fatto che in quegli anni quasi un quarto dell'intera superficie dell'Agro mutò di proprietario.

Gli acquirenti delle 78 tenute che la Giunta Liquidatrice mise all'asta e vendette tra il 1873 ed il 1881 furono circa 62: ciascuno di essi si aggiudicò una media di circa 750 ha, cioè meno della metà di quanto mediamente possedeva, nel 1870, ognuno degli enti ecclesiastici proprietari di quelle tenute. Di queste 78 tenute, soltanto 9 (pari a circa 2.879 ha) finirono nella mani di proprietari nobili ${ }^{16}$, mentre

15 La Legge 1402 del 1873 estese a Roma e al Lazio quel complesso di norme sulla liquidazione dell'asse ecclesiastico che già da qualche tempo era in attuazione nel regno d'Italia. Roma, però, fruì di un regime speciale: l'intera operazione fu, infatti, gestita da una Giunta Liquidatrice predisposta ad hoc, che svolse il proprio compito in maniera autonoma e rispondendone esclusivamente ad una Commissione di Vigilanza nominata appositamente dal Parlamento.

Anche per Roma, così come per il resto d'Italia, la legge fece una distinzione tra enti ecclesiastici da sopprimere - i cui beni lo Stato in parte destinò ad usi civili ed in parte vendette - ed enti ecclesiastici da conservare - ai quali lo Stato riconobbe una personalità giuridica ed ai quali impose di convertire le proprietà immobiliari in rendita pubblica.

16 Roberto Della Seta nel suo La liquidazione dell'asse ecclesiastico nell'Agro Romano, pubblicato su «Storia urbana», n. 40, lug.-set., Roma 1987, ricorda che i 
i restanti 39.000 e più ettari andarono tutti ad ingrossare la quota della proprietà borghese, e la gran parte di essi l'acquistarono, insieme ad alcuni esponenti della classe dirigente risorgimentale, $\mathrm{i}$ «mercanti di campagna». ${ }^{17}$

| «mercanti» di più antica tradizione che già possedevano della terra, però, non effettuarono nessun acquisto; furono soprattutto uomini nuovi a proporsi sulla scena romana, uomini che spesso accomunavano all'agricoltura attività economiche di tipo diverso e che, soprattutto, erano legati alla nuova classe dirigente piemontese.

La proprietà assunse, dunque, nuove forme economiche e giuridiche, passando dagli aspetti di tipo più arretrato a forme più moderne legate alle banche e alle società imprenditoriali (proprietà capitalistica), e molti proprietari terreni, piuttosto che dedicarsi all'attività agricola, preferirono dedicarsi all'attività più proficua di "costruire Roma".

Lo sviluppo della città procedette in maniera costante, e in alcune occasioni molto velocemente, verso il territorio agricolo senza vincoli e controlli e in maniera del tutto irrazionale. Tanto il Piano del 1909 che quello del 1931 rappresentarono, come vedremo nei paragrafi successivi, solo un tentativo ex-post di dare un senso razionale allo sviluppo caotico dettato dalle immobiliari, dalle lottizzazioni abusive e dagli interessi dei grossi proprietari che sempre hanno rappresentato una peculiarità della storia dell'area romana.

I valori dei suoli agricoli lievitarono, dunque, sia in relazione allo sviluppo della città e alla trasformazione della rendita fondiaria in rendita urbana, sia per effetto della bonifica dell'agro.

II Regio Decreto emanato nel 1878 istituì, così come sarà illustrato più dettagliatamente nel paragrafo successivo, l'obbligo per i proprietari dei terreni dichiarati insalubri di costituirsi in consorzio al fine di operare

nobili che divennero proprietari di queste 9 tenute ecclesiastiche furono: il duca Leopoldo, il principe Alessandro Torlonia, il duca Pio Grazioli, il conte Bosio Sforza Cesarini, il principe Giovanni Andrea Colonna, il marchese Napoleone Del Gallo di Roccagiovane, il principe Placido Gabrielli e il conte Giacomo Lovatelli. 
alla bonifica del territorio attraverso il prosciugamento delle paludi e degli scoli. Successivamente, nel 1933, un Decreto Legge svincolò la bonifica da un mero concetto idraulico, e, avendo come obiettivo soprattutto il massimo sviluppo delle attività agricole, portò da una parte ad un accrescimento degli investimenti in agricoltura, e ad un imponente frazionamento delle grandi proprietà dall'altra. Questo processo comportò, fondamentalmente, un arricchimento della grande proprietà capitalistica che vide crescere le estensioni di proprietà delle società per azioni da 3.000 ettari nel 1914 a ben 14.000 ettari nel 1940 .

Dalle operazioni della bonifica il territorio comunale usciva modificato, la proprietà nobiliare si restringeva di $34.000 \mathrm{Ha}$ controllando il $30 \%$ dei suoli $(57.000 \mathrm{Ha})$; il restante territorio andava concentrandosi nella mani della borghesia. $^{18}$

L'ultimo conflitto bellico accrebbe ulteriormente il potere dei proprietari terrieri, sia a causa della svalutazione monetaria, sia per la forte richiesta di aree fabbricabili dovuta alla stasi bellica e alla consistente ondata di immigrazione che arrivò nella capitale.

Il Comune fu ancora una volta assente nell'acquisizione delle aree e nel predisporre un piano che desse indirizzi per uno sviluppo equilibrato che potesse calmierare il mercato dei suoli. Ecco allora quello che fu chiamato "sacco di Roma"19. I grandi proprietari, nobili, borghesi, o gli stessi pastori divenuti proprietari, effettuarono soprattutto delle consistenti lottizzazioni dei loro terreni e si lanciarono, in parte, nel reimpiego di quanto ricavato dalle vendite per la costruzione di nuovi edifici.

Nelle campagne, inoltre, i braccianti e i salariati cominciarono a rivendicare le terre incolte e/o mal coltivate dei latifondi, fino ad ottenere negli anni fra il '47 e il '50 la creazione dell'Ente Maremma, che si

18 O. MANCINI, op. cit., p.268.

19 L'argomento è ampiamente trattato in P. DeLLA SETA, R. DELLA SETA, I suoli di Roma. Uso e abuso del territorio nei cento anni della capitale, Editori Riuniti, Roma 1988. 
occupò di assegnare le terre espropriate ai vecchi proprietari - in parte indennizzati per le perdite subite - ai braccianti e ai contadini che ricevettero una quota pari a circa 2,5 ettari ciascuno.

La paura degli espropri fu la ragione che pian piano determinò la nascita di numerose società immobiliari, portando nel biennio '64-'66 ad una nuova frammentazione dei terreni agricoli, tanto che la proprietà al di sopra dei 100 ha si ridusse del 31,6\%, le proprietà comprese tra i $30 \mathrm{e}$ i 100 ha raddoppiarono la loro superficie e il rimanente $22,6 \%$ del territorio si frammentò al di sotto dei 30 ha.

\section{LE OPERE DI BONIFICA}

L'azione anti-malarica nell'Agro Romano fu materia di interventi fin dal XVII secolo, in quanto l'insalubrità delle aree paludose aveva portato nei secoli ad uno spopolamento della campagna e ad una forte difficoltà di sopravvivenza per i lavoratori stagionali.

Tale caratteristica del territorio intorno a Roma, soprattutto a sud della città, nasce dal fatto che si tratta di una sorta di depressione costiera circondata da circa 14.000 ettari di superficie collinare. Le pendenze scolavano sui terreni posti al di sotto o appena al di sopra del livello del mare, acque che si andavano a sommare agli stagni e agli acquitrini già presenti sul litorale, creando in questo modo problemi che superavano il loro "semplice" prosciugamento. Per attuare il drenaggio di queste zone si cercò, innanzitutto, un metodo efficace e risolutivo attraverso il quale separare le acque alte, provenienti dai terreni collinari, dalle acque basse, contenute negli stagni e derivanti dalle piogge e dalle sorgive sotterranee presenti.

Roma dipendeva dalle derrate alimentari prodotte nella sua campagna e i pontefici avevano già pensato ai provvedimenti necessari per una produttività agricola maggiore e, soprattutto, continua. A vari 
tentativi pontifici, però, si contrapponeva in maniera radicale la mancanza di iniziativa e di interventi da parte della nobiltà latifondista romana che premeva - come abbiamo - verso un'economia agraria basata su rapporti lavorativi precari e discontinui.

Lo spopolamento, il dissesto del regime idraulico e la malaria restarono così ancora per quasi tutto l'Ottocento gli elementi dominanti dell'Agro Romano. ${ }^{20}$

Con I'Unità d'Italia il progetto per il risanamento ed il ripopolamento dell'Agro fu ripreso e l'interesse per la bonifica del litorale da parte del governo sabaudo fu immediato: sin dall'ottobre del 1870 furono infatti nominate diverse commissioni alle quali venne affidato il compito di studiare le paludi presenti presso la foce del Tevere e di proporre soluzioni per arrivare ad una completa bonifica delle stesse. Nel marzo del 1873 venne presentato da una commissione tecnica del Genio Civile un primo progetto di massima per il bonificamento degli stagni di Ostia e Maccarese.

Dopo un'altra serie di studi e di proposte progettuali, nel 1878 ebbe inizio il periodo della promulgazione delle leggi sulla bonifica dell'Agro Romano. La prima legge, la n. 4642 dell'11 dicembre 1878, aveva come obiettivo generale quello del miglioramento igienico della città e della campagna di Roma. In questa legge il punto centrale fu la dichiarazione di pubblica utilità del bonificamento dell'Agro, cosa che comportò da parte dello Stato l'assunzione di tutti gli oneri finanziari. La L. 4642, fondamentalmente, prevedeva: il prosciugamento delle paludi e degli stagni di Ostia e Maccarese, del Lago dei Tartari, delle paludi di Stracciacappe, dell'Almone, di Pantano e di Baccano; l'incanalamento e lo scolo di tutte le acque stagnanti; la bonifica di una fascia di terra di circa $10 \mathrm{~km}$ intorno al centro di Roma. Quasi tutte le operazioni, come

20 A. Parisi Presicce, G. Villetti, Le bonifiche: un ponte fra passato e futuro, in C. BAGNASCO (a cura di), «ll delta del Tevere: un viaggio fra passato e futuro», F.lli Palombi, Roma 1998, p.97. 
detto, erano a carico dello Stato, mentre ai proprietari terrieri di quella zona, che per legge erano obbligati a riunirsi in consorzi, spettava di:

- fare e mantenere in ciascun consorzio i canali e i fossi principali dell'allacciamento e di scolo;

- procurare nelle singole proprietà, raccolte nei consorzi, l'allacciamento ed il deflusso di tutte le acque stagnanti e sorgive.

L'unica voce che si occupava della bonifica agraria era quella che prevedeva che i beni, ricadenti in area malarica, degli enti ecclesiastici soppressi

potevano essere dati in enfiteusi in lotti «non maggiori di ettari 400» per pubblico incanto. $^{21}$

Nel 1883, poi, venne emanata una seconda legge - la n. 1489 dell'8 luglio 1883 - che, nella sostanza, rappresentava una sorta di specificazione ed attuazione della precedente. La novità più evidente fu quella di rendere esplicito l'obbligo per i proprietari di bonificare i terreni compresi nei $10 \mathrm{~km}$ intorno alla città e di presentare un progetto circa $\mathrm{i}$ miglioramenti agrari da apportare; questi progetti dovevano essere poi esaminati dal Ministero dell'Agricoltura, cui spettava il compito di dare il proprio assenso o rifiuto alle proposte e di notificare a ciascun proprietario le opere da eseguire e i tempi entro i quali ciò doveva avvenire. Gli inadempienti potevano, a norma della legge generale di esproprio per pubblica utilità, venire espropriati delle proprie terre le quali, successivamente, o venivano date in affitto, o venivano vendute all'asta. Come misura d'incoraggiamento vi erano un'esenzione di dieci anni dalle imposte sui fabbricati ed una di vent'anni per l'aumento di reddito conseguente ai miglioramenti approntati. Questa normativa, in realtà, ricadeva su poco meno di un centinaio di tenute, quante cioè erano le proprietà che rientravano nel raggio dei $10 \mathrm{~km}$ dal centro di Roma.

21 L. Bortolotti, Roma fuori le mura, Laterza, Roma - Bari 1988, p.87. 
In realtà le leggi emanate dallo Stato per bonificare l'Agro Romano erano di gran lunga più vantaggiose per i proprietari di quanto non lo fossero quelle nazionali sulle bonifiche. Secondo la legge generale, infatti, le bonifiche si distinguevano in due categorie: quelle di I categoria erano eseguite dallo Stato col concorso, per circa metà della spesa, delle Province, dei Comuni e dei proprietari, ai quali spettava la manutenzione; quelle di II categoria erano, invece, totalmente ed esclusivamente a carico dei privati.

È da notare come di fatto lo scopo principale delle leggi, tanto quelle generali per il territorio nazionale, quanto quelle specifiche per l'area romana, fu la bonifica idraulica, mentre fu sempre messa in secondo piano - o addirittura rimandata ad un secondo tempo - la bonifica agraria. II motivo principale risiedeva nel fatto che quest'ultima comportava, il più delle volte, la messa in crisi di un sistema proprietario radicato storicamente e, quindi, difficile da intaccare.

Nel 1903 venne intanto emanata una nuova legge per l'Agro - L. 474 del 13 dicembre 1903 - che stabilì nuove esenzioni ed introdusse la novità dei mutui a tasso ridotto per i avori di bonifica eseguiti dai privati. Nel 1905, poi, venne predisposto un Testo Unico - R.D. 647 del 10 novembre 1905 - che coordinava tutte le leggi fino ad allora emanate, mentre cinque anni dopo venne emanata una nuova legge - L. 491 del 17 luglio 1910 - che predispose alcune importanti innovazioni. La più importante fu la costruzione dei «centri di colonizzazione», per i quali vennero stabilite alcune condizioni di favore e l'espropriazione forzata dei terreni occorrenti.

II Regolamento della legge del 1910 - R.D. 248 del 22 gennaio 1911 - attraverso il quale veniva approvato il regolamento concernente i provvedimenti per la bonifica e la colonizzazione dell'Agro Romano, fece poi ancora qualche passo avanti per ciò che interessava sia la bonifica, sia i centri. Per quanto riguarda la prima il decreto regio rese possibile l'adesione volontaria dei proprietari non più obbligati per legge; per ciò 
che interessava i centri, parallelamente, vennero stabilite le caratteristiche che dovevano avere le costruzioni nelle borgate rurali e si abilitarono i Comuni, le Province e le istituzioni di beneficenza a costruire borgate, ammettendo anche il loro ampliamento oltre i confini originari e ponendo in questo modo le premesse ad un loro non troppo lontano passaggio da rurali ad urbane.

In senso analogo va il decreto luogotenenziale - D.L.Luog. 662 del 24 aprile 1919 - che ben nove anni dopo, risentendo dell'atmosfera politica del cosiddetto «biennio rosso», dà un peso più forte agli interessi comuni e si dimostra meno acquiescente nei confronti della proprietà privata. Con questa legge, infatti

- viene ammessa ai benefici stabiliti dalla legislazione precedente anche la costruzione di centri destinati a famiglie non addette all'agricoltura;

- viene indicata come condizione di preferenza, per le borgate, la prossimità di strade o di stazioni ferroviarie o tranviarie;

- vengono obbligati i proprietari delle aree in corso di bonifica a fornire i locali occorrenti per le scuole rurali e gli ambulatori.

Di lì a pochi mesi gran parte dei provvedimenti legislativi approntati per la bonifica della campagna romana venne estesa a molte altre regioni d'Italia.

I principali momenti legislativi in materia della bonifica e colonizzazione della campagna romana si possono, quindi, così sintetizzare:

\begin{tabular}{|l|l|l|}
\hline anno & \multicolumn{1}{|c|}{ procedimento leg.vo } & \multicolumn{1}{c|}{ argomento } \\
\hline 1878 & Legge 4642 & $\begin{array}{l}\text { Miglioramento igienico della capitale e della } \\
\text { campagna romana per interesse della nazione }\end{array}$ \\
\hline 1883 & Legge 1489 & Bonificamento dell'Agro Romano \\
\hline 1903 & Legge 474 & Bonificamento dell'Agro Romano \\
\hline 1905 & Regio Decreto 647 & $\begin{array}{l}\text { Approvazione del T.U. delle Leggi sul } \\
\text { bonificamento dell'Agro Romano e sulla } \\
\text { colonizzazione dei beni demaniali dello Stato }\end{array}$ \\
\hline 1910 & Legge 491 & $\begin{array}{l}\text { Provvedimenti per estendere il bonificamento } \\
\text { dell'Agro Romano }\end{array}$ \\
\hline 1911 & Regio Decreto 248 & Regolamento concernente i provvedimenti per il \\
\hline
\end{tabular}




\begin{tabular}{|l|l|l|}
\hline & & $\begin{array}{l}\text { bonificamento e la colonizzazione dell'Agro } \\
\text { Romano }\end{array}$ \\
\hline 1919 & Decreto Legge Luog. 662 & $\begin{array}{l}\text { Nuovi provvedimenti per la bonifica dell'Agro } \\
\text { Romano }\end{array}$ \\
\hline
\end{tabular}

Il provvedimento preso dal governo che ebbe effetti più precisi fu, comunque, la bonifica idraulica vera e propria, soprattutto perché realizzata, come abbiamo già avuto modo di sottolineare, quasi interamente a spese della collettività e non dei proprietari terrieri.

Per risolvere il problema tecnico furono inizialmente proposte due possibili soluzioni progettuali: la prima prevedeva la colmata delle depressioni, la seconda il prosciugamento mediante mezzi idraulici delle acque stagnanti. Più specificatamente, nel primo progetto veniva proposto di colmare le terre poste sotto il livello del mare con i detriti portati, con opportune deviazioni, dal fiume Tevere: si trattava, in sostanza, di utilizzare il principio naturale che era alla base della formazione della zona del delta tiberino. Un simile procedimento comportava, però, tempi di attuazione particolarmente lunghi, sull'ordine di circa una decina d'anni.

La soluzione idraulica della seconda proposta, invece, si basava sulla costruzione di canalizzazioni che drenassero le acque basse e le convogliassero in una vasca d'arrivo; da questa, un impianto idrovoro avrebbe provveduto a sollevarle e scaricarle direttamente in mare. Questo sistema parve più economico e veloce del primo, tanto che I'ufficio tecnico del Genio Civile decise di procedere in questo senso ed il progetto per il prosciugamento meccanico delle paludi di Ostia, Maccarese ed Isola Sacra fu approvato il 15 luglio del 1880.

La Società Canzini Fueter \& Co. fu la principale impresa che si aggiudicò i lavori con un contratto, stipulato nell'aprile 1884, che prevedeva una durata delle operazioni di bonifica di 48 mesi, compresi quelli durante i quali si dovevano sospendere le attività per il periodo malarico. In realtà tanto le stime economiche, che quelle temporali 


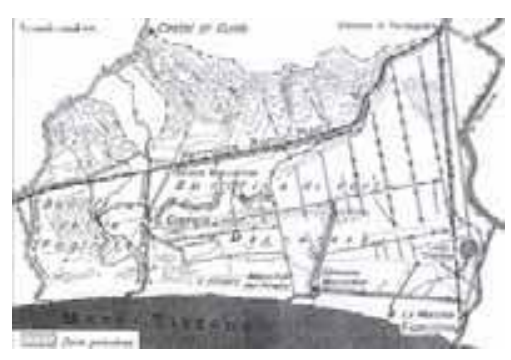

Figura 4. La zona di Maccarese prima della bonifica.

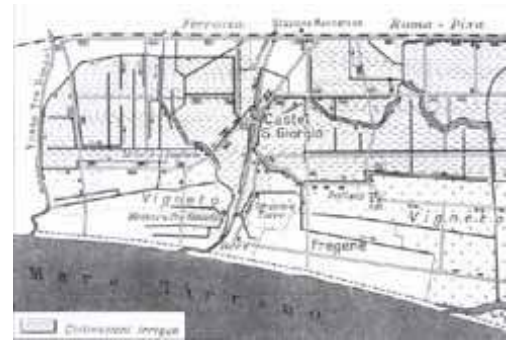

Figura 5. La zona di Maccarese dopo la bonifica. andarono deluse ed i costi si rilevarono più alti, così come i tempi di realizzazione furono molto più lunghi.

Tra le maestranze che parteciparono concretamente all'opera di bonifica dell'Agro occorre ricordare l'apporto costante dell'Associazione generale operaia braccianti del Comune di Ravenna, che ottenne in subappalto nel 1884 i lavori per il bonificamento delle zone paludose. I ravennati non furono, però, l'unico gruppo di lavoratori impegnati nei movimenti di terra; ad essi si affiancarono presto piccoli gruppi di abruzzesi e marchigiani, impiegati nelle operazioni relative allo scavo dei canali, alla costruzione degli argini e degli impianti idrovori, ecc.

Il prosciugamento delle paludi iniziò, dunque, con il separare le acque alte da quelle basse e con l'arginare il fiume Tevere che, nei periodi di piena, contribuiva ad allagare a sua volta gli stagni. Per prima cosa si procedette alla costruzione del canale di Dragoncello e della Lingua nell'area dell'Ostiense, che portò ad isolare lo stagno ostiense dai versamenti di acqua provenienti dalle zone collinari alle spalle di quest'ultimo: in questo modo le acque che scorrevano al di sopra del livello del mare riuscivano a raggiungere la spiaggia in modo naturale. Con un sistema siffatto veniva evitato sia il rigonfiamento dello stagno nei mesi piovosi, sia di andare a gravare sulle idrovore con un lavoro supplementare che rischiava di comprometterne il funzionamento. Un procedimento analogo venne applicato alla zona di Maccarese, dove vennero costruiti i canali allaccianti del fosso Galeria e di Maccarese.

Contemporaneamente fu avviata la costruzione delle macchine idrovore che, però, funzionarono solo dalla fine del 1889, a causa delle difficoltà che insorsero nella loro costruzione per la differente natura del terreno che non sempre reggeva uniformemente il peso soprastante. Vennero adottati, quindi, particolari tipi di fondamenta per ottenere in tutti i luoghi l'effetto desiderato. Nonostante le difficoltà e i ritardi riscontrati - dovuti soprattutto alla tecnica costruttiva che mancava ancora di esperienza nel settore - è importante notare come ad oggi le 
soluzioni adottate si siano rivelate le migliori, a tal punto che le strutture portanti degli impianti idrovori sono ancora quelle originali.

L'ultima fase della bonifica dell'Agro iniziò dopo il 1889, con il vero e proprio svuotamento degli stagni.

A questo punto la macchina $d$ era completamente e definitivamente messa a regime: ormai sollevate dalle idrovore, le acque correvano verso il mare attraverso la rete dei canali emissari. Lo scavo di colatoi e collettori delle acque basse assunse la classica, e ancor oggi ben visibile, forma a spina di pesce:

i canali più piccoli confluivano in quelli più grandi e questi a loro volta nei collettori primari, che terminavano nella vasca d'arrivo; la quale, posta nel punto più basso della depressione, raccoglieva le acque per essere quindi sollevate dalle idrovore. ${ }^{22}$

Va sottolineato che il Governo si limitò ad attuare una legislazione di compromesso fra gli interessi dei latifondisti e l'interesse pubblico, tanto nella formulazione delle leggi, quanto nella loro effettiva applicazione. Gli obblighi dei proprietari, infatti, che nella prima legge per la bonifica abbiamo visto relativi alla loro partecipazione ai consorzi e al deflusso per allacciamento delle acque stagnanti nelle proprietà, furono generalmente elusi. La stessa cosa avvenne per la seconda legge del 1883, che riguardava sia il bonificamento idraulico che la vera e propria bonifica agraria, che minacciava di espropri gli inadempienti e che, alla fine, fu ben poco applicata e in qualche caso finì addirittura per premiare coloro i quali dovevano essere puniti.

La legge del 1883, come detto, aveva reso obbligatoria per i proprietari la bonifica su 43.803 ettari, ma alla fine del secolo quasi nulla era stato ancora fatto: nel 1898 solo tre tenute erano state espropriate per inadempienza alle prescrizioni ministeriali. La lentezza con la quale

22 G. LATTANZI, V. LATTANZI, La bonifica del litorale di Roma, 1870-1911, in C. Aymonino (a cura di), «Architettura e urbanistica. Uso e trasformazione della città storica», Marsilio, Venezia 1984, pp.145-146. 
procedeva lo Stato nell'eseguire il prosciugamento degli stagni era il pretesto per i consorzi per non agire, e questo loro immobilismo era il pretesto per i proprietari per non fare gli interventi che spettavano loro per legge.

La situazione in parte cambiò dopo l'inizio del XX secolo, soprattutto a seguito dell'introduzione delle esenzioni e dei mutui di favore e di alcuni obblighi introdotti dal Comune a seguito della legge del 1903. Alla fine del 1909, infatti, sui 43.808 ettari che costituivano allora la zona soggetta alla bonifica agraria obbligatoria, per circa 41.950 esistevano progetti di miglioramento agrario approvati e resi obbligatori.

Quello che si tentava di imporre con le nuove leggi in vigore era la divisione delle tenute dell'Agro in più unità, in maniera tale da poter introdurre un'agricoltura più moderna. Ciò fu in realtà perseguito in maniera molto blanda: l'allevamento delle pecore continuava ad essere più conveniente delle colture intensive auspicate, tenendo ancora molto bassa la densità demografica nelle aree che non erano investite dallo sviluppo urbano.

\section{L'INFRASTRUTTURAZIONE DEL TERRITORIO}

La rete stradale prima del 1870 era quasi esclusivamente rappresentata dalle 18 radiali che per lo più ricalcavano i percorsi delle antiche strade consolari ${ }^{23}$ e che da Roma raggiungevano le località più 0 meno vicine alla città: queste strade generalmente in pessimo stato, e che certo non rappresentavano una rete costruita al servizio della campagna, comunque permettevano un minimo di scambi fra città e

23 Procedendo in senso orario, partendo dal Tevere, le radiali erano: strada della Magliana, via Portuense, via Aurelia, via di Boccea, via Trionfale, via Cassia, via Flaminia, via Salaria, via Nomentana, via Tiburtina, via Prenestina, via Casilina, via Tuscolana, via Appia nuova, via Appia antica, via Ardeatina, via Laurentina, via Ostiense.

(Maggiori informazioni su questi temi possono essere desunte da L. BORTOLOTTI, op. cit.). 
Agro. Non bisogna dimenticare, infatti, che nell'Ottocento le strade rivestivano un'importanza assai ridotta rispetto alle ferrovie e, in alcune zone e casi, anche rispetto ai canali navigabili. Vi era addirittura una Legge sui lavori pubblici che non ammetteva l'esistenza di strade nazionali che ricalcassero lo stesso percorso delle ferrovie e che comportò - come risultato più evidente - una forte diminuzione delle strade statali ed un loro declassamento e abbandono.

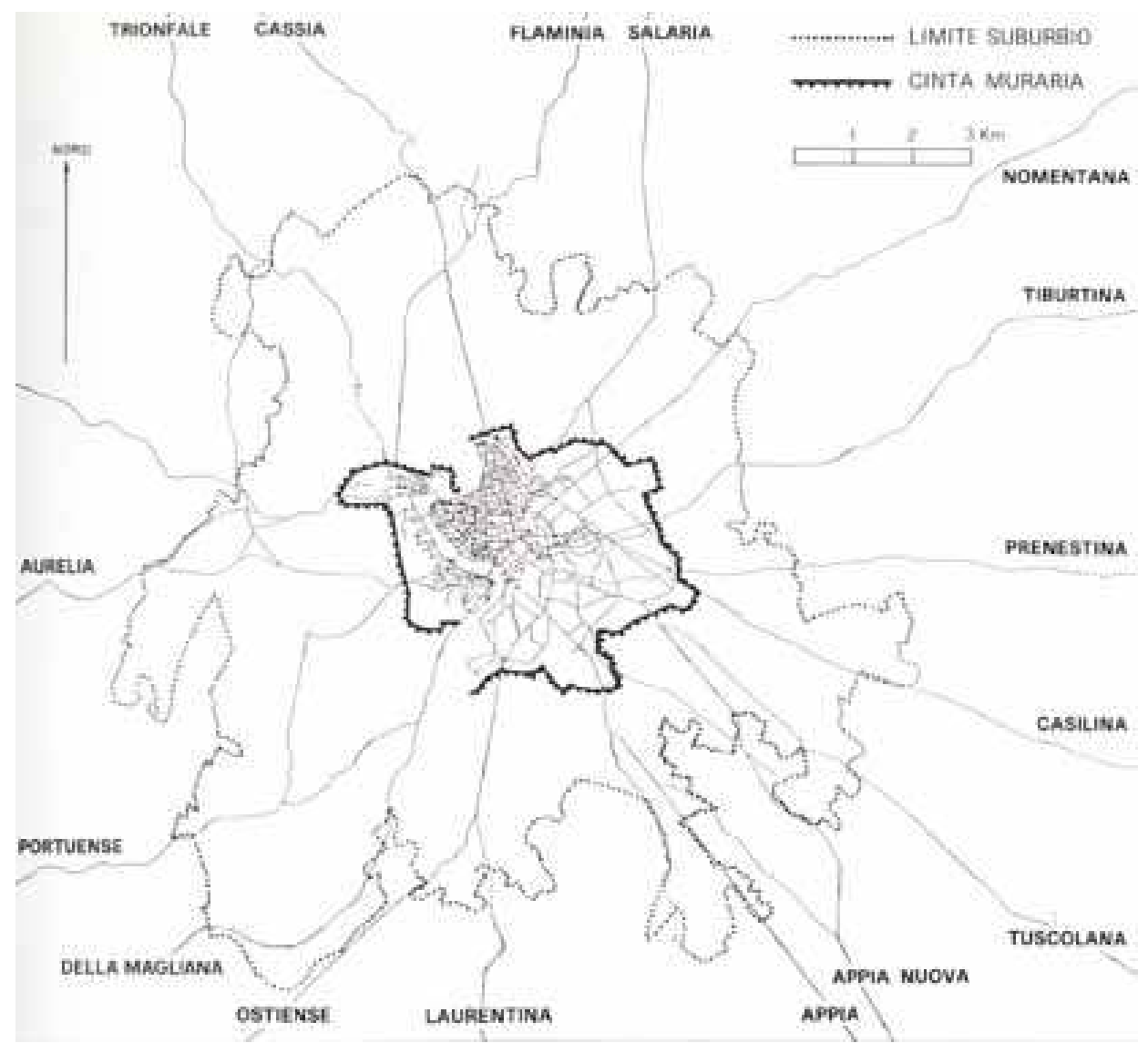

Figura 6. La rete viaria, rispetto alla cinta muraria ed al suburbio delle «vigne», nel 1870.

La crescita di Roma impose ben presto dei cambiamenti, sia per ciò che riguardava le antiche consolari in vicinanza delle porte della città, sia per l'intero reticolo infrastrutturale quasi del tutto assente nella campagna. Gli ampliamenti e rettifiche delle radiali, così come la 
costruzione di nuovi ponti sul Tevere, riguardarono direttamente l'estensione e l'attrezzatura della città, per collegarla al suo immediato suburbio e per rendere più agevole il passaggio tra le due sponde del fiume.

Intorno ai primi anni del novecento si assisté poi, anche nella campagna, ad un miglioramento, e presto si moltiplicarono le strade. I proprietari terrieri messi sotto torchio da leggi e regolamenti comunali sempre più pressanti e severi e, nello stesso tempo, aiutati da mutui statali e - in parte - comunali, non si mostrarono più ostili alla realizzazione delle strade nelle loro proprietà. In molti casi, anzi, furono i primi a reclamarle.

Un primo passo avanti fu fatto con la realizzazione del cosiddetto "campo trincerato": una cerchia di forti e batterie fortificate ${ }^{24}$ che, poste ad un intervallo di circa $2 \mathrm{~km}$, erano distanti dalla città dai 2 ai $4 \mathrm{~km}$ e che dovevano essere collegate a questa e tra loro in qualche modo. Vennero quindi realizzate alcune strade che all'inizio erano chiuse ai civili, ma che presto, quando nel 1906 il governo decise di liberalizzarle, divennero uno degli elementi strutturanti l'organizzazione complessiva del territorio intorno alla città.

La costruzione nel suburbio di alcune grandi attrezzature urbane, così come il dettato della legge del 1903 per le opere di bonifica dell'Agro, o come il rafforzato servizio delle ambulanze dalla campagna agli ospedali della città, richiesero una rete della viabilità più fitta e con fondi stradali migliori e comportarono un maggior dinamismo da parte dell'Amministrazione Comunale.

Negli stessi anni nascevano le prime autolinee extraurbane, con il servizio pubblico automobilistico che copriva la linea Roma Campagnano - Nemi e con quello che andava da piazza Venezia ed Ostia: esse rappresentarono il primo esempio della politica statale e

24 I forti e le batterie compresi in questa organizzazione erano: il forte di Pietralata, il forte Tiburtino, il forte Prenestino, la batteria di Porta Furba, il forte Casilino, la batteria dell'Acquasanta, il forte Appio e il forte Ardeatino. 
comunale di incoraggiamento al trasporto su strada che perdura ai giorni nostri.

Con un notevole salto temporale si arriva all'inizio degli anni '50, quando una consistente fascia di campagna romana intorno alla città fu interessata dai lavori per i primi tronchi stradali dell'arteria del Grande Raccordo Anulare 25 .

La costruzione del G.R.A. è durata all'incirca 25 anni, fra progettazione, realizzazione, interventi successivi di completamento e ristrutturazione, ...

II Raccordo Anulare che cinge la città di Roma ha assunto per interventi successivi la sezione attuale, che in alcuni tratti ha una sede stradale costituita da tre corsie, più una di sosta d'emergenza, per ogni senso di marcia, in altri tratti - soprattutto quelli relativi al settore urbano orientale - da due corsie di marcia. È attualmente in corso un ampi operazione di ristrutturazione delle parti di raccordo più sacrificate entro un contesto urbano denso ${ }^{26}$, per le quali l'ANAS sta procedendo alla realizzazione della terza corsia.

Intanto sul finire degli anni Sessanta la motorizzazione privata è cresciuta in modo notevole e comincia a produrre i primi guasti: nel 1970 circolano 970.000 autovetture e ogni anno se ne aggiungono 100.000; la rete stradale è cresciuta

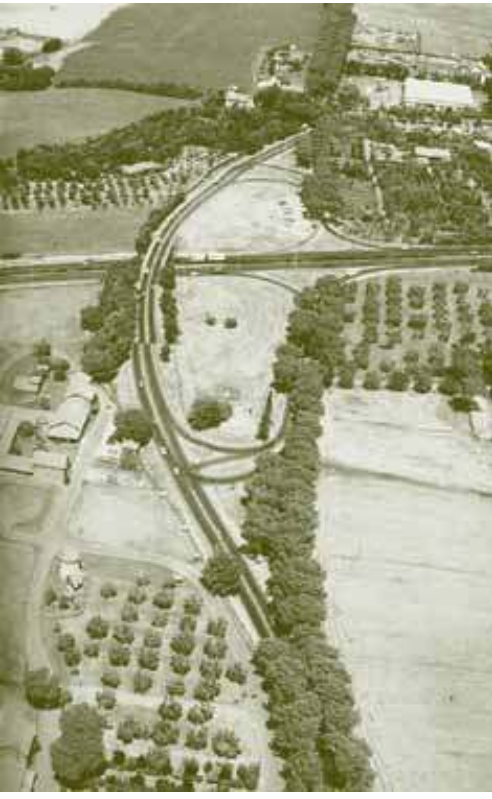

Figura 7. Svincolo del GRA tra scorci di campi coltivati all'incrocio con la via Nomentana. fino a 1.200 chilometri, ma cominciano i grandi ingorghi nelle aree centrali $[\ldots]^{27}$

25 II Grande Raccordo Anulare è meglio conosciuto nella sua forma abbreviata G.R.A.; tale abbreviazione coincide con il cognome del progettista ed esecutore dell'intera operazione.

26 II Raccordo, come la grande maggioranza delle infrastrutture viarie moderne, sembra essere stato "appiccicato" al territorio in cui è inserito, senza avere con questo nessun tipo di relazione. A tratti attraversa la campagna romana, ed è possibile coglierne ancora qualche lembo di appezzamenti coltivati o qualche rudere di storia; in altri tratti, invece, bordeggia l'urbanizzato delle espansioni degli anni '70 e diventa quasi una strada urbana, assalita dal traffico e dal caos. 
Alla pressione della motorizzazione, infatti, l'amministrazione pubblica (comunale e statale) risponde con la realizzazione di un gran numero di infrastrutture viarie: è in questo periodo che il sistema autostradale di accesso alla città raggiunge il suo assetto quasi definitivo.

Nel 1962 viene aperta al traffico l'autostrada Roma - Napoli; l'anno successivo si inaugura il tratto nord dell'autostrada del sole fino a Magliano Sabina; fra il 1968 ed il 1970 si apre il primo tratto dell'autostrada per l'Abruzzo. Vengono inoltre ampliati gli accessi urbani di via Aurelia, via Tiburtina e via Anagnina; nel 1964 cominciano i lavori per la costruzione delle due carreggiate laterali della via Cristoforo Colombo, fra Roma e il quartiere dell'EUR.

Nel settore ovest della città via Baldo degli Ubaldi viene collegata alla via Olimpica e nel 1965 viene completata la strada «panoramica» che va da piazzale Clodio a Monte Mario. A nord vengono realizzati il viadotto di via delle Valli che sovrappassa il fiume Aniene, il raddoppio della via Nomentana, la penetrazione di Pietralata, dando origine, in questo modo, ad un fitto sistema di radiali di connessione fra le aree più centrali della città e le nuove espansioni urbane del nord-est (Monte Sacro, Parti Fiscali, Talenti, ecc...). Nel settore orientale, infine, viene completato il cavalcavia sulla ferrovia Roma - Tivoli, a completamento della tangenziale est.

Per quel che riguarda la rete ferroviaria, già nel 1870 esisteva nel Lazio una serie di collegamenti incentrati sulla città ${ }^{28}$ : c'erano la linea per Ciampino e Frascati aperta nel 1857; quella per Civitavecchia del 1859; la Ciampino - Velletri aperta nel 1862 e prolungata negli anni

28 (...) sotto il regime papale si guarda alle ferrovie come ad uno strumento per consentire soprattutto agli abitanti dei colli Albani e Tuscolani di far affluire i loro prodotti sul mercato di Roma.

(G. ASCARELLI, Ferrovie e tramvie nello sviluppo dei trasporti urbani ed interurbani, in C. AYMONINO (a cura di), «Architettura e urbanistica. Uso e trasformazione della città storica», Marsilio, Venezia 1984, p.135). 
successivi in direzione Napoli fin oltre Cassino; la Roma - Orte aperta nel 1864 e prolungata in seguito verso l'Umbria.

La Roma papale degli ultimi anni era già collegata col resto d'Italia: nel 1863 si poteva andare per ferrovia da Roma a Napoli; nel 1867 a Livorno e Pisa; nel 1866, attraverso l'Umbria, sia a Falconara Marittima sull'Adriatico, che a Firenze. Nel 1872 poi si aprì la linea diretta da Roma a Firenze attraverso Orvieto e Chiusi. ${ }^{29}$

Quando il Lazio fu annesso al Regno d'Italia, i grandi lavori ferroviari erano, dunque, in corso da anni e in poco tempo furono completati: le grandi linee che convergevano sulla capitale servivano però luoghi importanti e lontani, più che la vicina campagna.

Furono, infatti, più utili per l'Agro le linee minori che venivano in quegli anni progettate: nel 1878 fu costruita la linea che collegava Roma al porto di Fiumicino, staccando un tronco della Roma - Pisa all'altezza di Ponte Galeria; l'anno successivo fu inaugurato il collegamento - in un primo tempo effettuato mediante una tramvia a vapore - tra Roma e Tivoli, con un percorso che in parte era sulla Tiburtina, in parte in sede propria. A questa tramvia per Tivoli si aggiunse, nel 1887, una vera e propria ferrovia con un percorso più a sud, lungo la quale, nelle immediate vicinanze della stazione Prenestina, sorsero alcune importanti industrie.

I programmi delle linee ferroviarie e tramviarie che all'inizio erano stati concepiti ed intrapresi per il trasporto di merci (derrate alimentari soprattutto) dai centri minori del Lazio verso la città, ben presto vennero dunque soppiantati da quelli per il trasporto passeggeri per e da Roma, soprattutto per spostare una consistente massa di manodopera.

Notevole rilievo, in questo senso, assunse la realizzazione della linea per i Castelli, cioè il collegamento tra Roma e i paesi e le cittadine dei Colli Albani. Fu una vicenda particolarmente lunga e complicata, che si concluse nel 1889 con l'inaugurazione del percorso Ciampino -

29 L. BORTOLOTTI, op. cit., p.122. 
Albano - Marino che, in parte, riutilizzava un vecchio tratto ferroviario che andava da Roma a Marino.

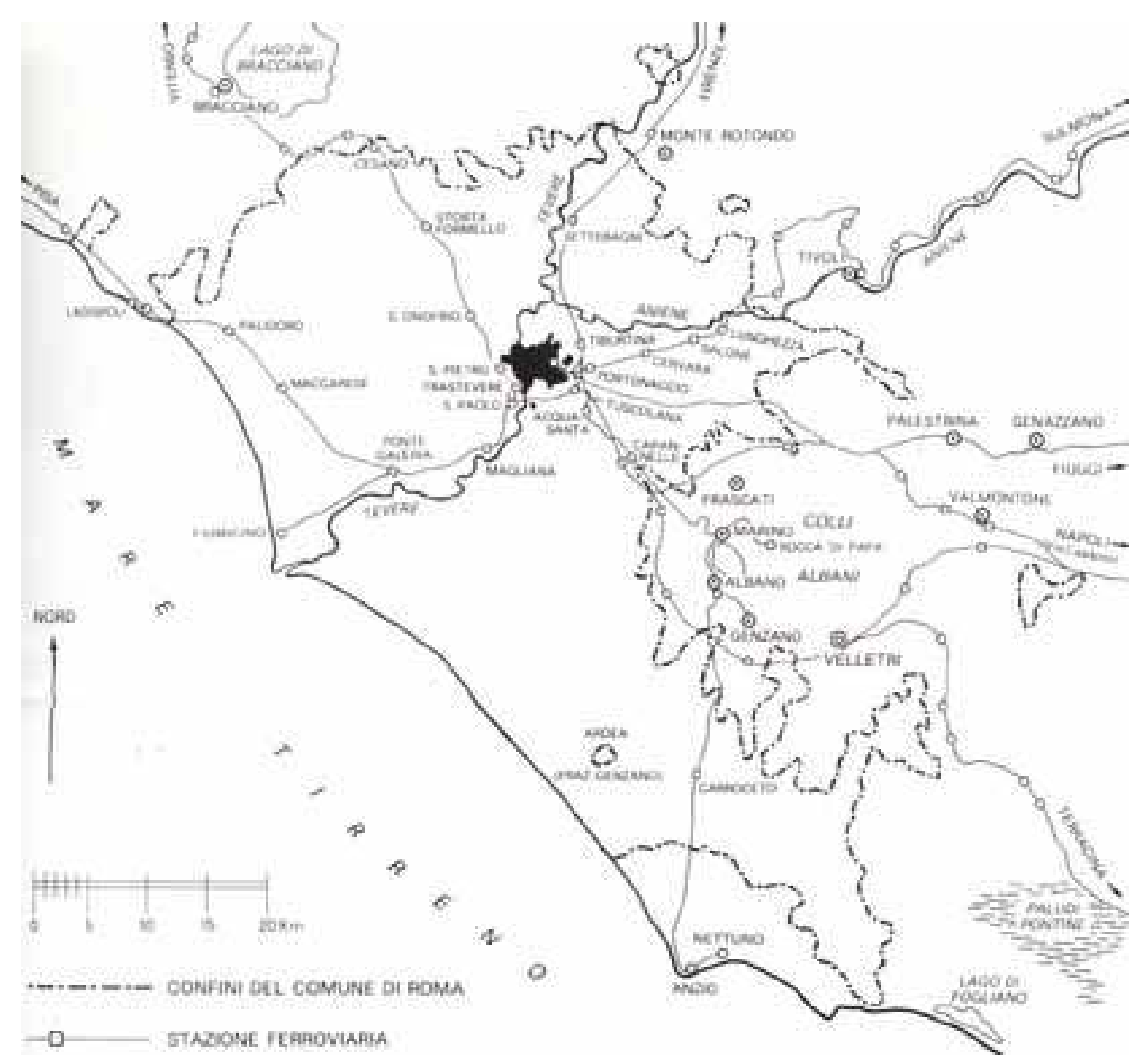

Figura 8. Linee e stazioni ferroviarie all'inizio del XX secolo.

Nel 1892, poi, venne aperta la linea Ciampino - Palestrina che costeggiava a nord i monti laziali; nel 1894 si inaugurò la Roma Trastevere - Viterbo, che passava per Bracciano; nel 1906 vi si aggiunse una tramvia che, seguendo un percorso diverso, costeggiava la via Flaminia e che venne sostituita solo nel 1932 da una ferrovia che arrivava fino a piazzale Flaminio, nel pieno centro di Roma. Nel 1916 venne, poi, inaugurata la ferrovia a scartamento ridotto per Fiuggi e Frosinone e nel 1924 nacque la Roma - Ostia. 
Tra il 1890 e il 1911, infine, vennero realizzati tutti i complicati raccordi ferroviari tra le diverse stazioni della città e si effettuò lo spostamento di una di queste - Trastevere - in una posizione e con un ruolo più strategici.

Nel complesso, quando nel 1909 Ernesto Nathan divenne sindaco della capitale, su Roma convergevano otto linee ferroviarie ${ }^{30}$ con ben 22 stazioni presenti sul territorio romano: molte di esse erano poste nell'immediata periferia della città e per questo presto furono inglobate nell'espansione degli anni successivi. Ognuna, però, seguivana una propria logica, unendo i centri urbani per i percorsi più brevi e collocando le stazioni in luoghi che, rispetto ai centri dell'Agro, risultavano insignificanti e, oltre tutto, mal collegati.

Solo due linee, quelle di Frascati e quella di Tivoli, (...), consentivano comunicazioni facili ed economiche. Le stazioni di Marino e Albano sulla vecchia linea per Napoli erano ben lontane dai paesi; la linea di Fiumicino era scarsamente servita (...). ${ }^{31}$

Occorre tener presente che la geografia delle ferrovie, soprattutto alla fine dell'Ottocento, coincideva quasi interamente con quella della malaria: le ferrovie, per ovvi motivi legati alla facilità di realizzazione, percorrevano infatti $\mathrm{i}$ fondovalle e le pianure dove la malaria imperversava e dove era più difficile effettuare qualsiasi altro tipo di collegamento.

Comunque, è certo che il contributo delle tramvie e ferrovie minori alla trasformazione della campagna romana fu notevole: il maggior popolamento dell'Agro avvenne, infatti, oltre che per le imponenti opere di bonifica, grazie all'entrata in funzione delle prime tramvie extraurbane, che concorsero a:

30 Roma - Fiumicino, Roma - Civitavecchia, Roma - Bracciano - Viterbo, Roma - Orte, Roma - Tivoli, Roma - Segni, Roma - Frascati, Roma - Albano (Cfr. L. BortolotTI, op. cit., pp.122-126).

31

L. BORTOLOTTI, op. cit., p.125. 
- $\quad$ togliere pian piano paesi e cittadine, posti a oltre $20 \mathrm{~km}$ dal centro di Roma, dall'isolamento che ancora alla fine del secolo caratterizzava il territorio intorno alla città papale;

- stimolare l'interesse per luoghi che risultavano ormai dimenticati e che rivestivano invece un grande significato storico ed archeologico;

- rendere accessibili zone che, soprattutto per ragioni orografiche e per l'assenza di infrastrutture, erano fino ad allora rimaste pressoché inaccessibili.

Fra le grandi opere nel settore delle infrastrutture non vanno dimenticate le vicende che hanno accompagnato la realizzazione dell'aeroporto intercontinentale di Fiumicino.

Già nel 1948 lo scalo aeroportuale di Ciampino si rivelava insufficiente, capace com'era di ospitare non più di 150 voli giornalieri. La scelta di localizzazione del nuovo aeroporto della capitale, però, non fu agevole, anche perché le aree deputate erano quelle abbastanza difficili per la loro natura della bonifica di Maccarese e di Porto. Furono stanziati nel 195514 miliardi ed i lavori cominciarono nel 1959 per concludersi un paio di anni dopo: i costi preventivato furono però largamente superati.

L'aeroporto, in un primo tempo, fu collegato con la città tramite l'autostrada Roma - Ostia che, però, ben presto si dimostrò del tutto insufficiente. Nel 1962 cominciarono i lavori per la nuova autostrada per Fiumicino e per Civitavecchia, che ancora oggi rappresenta uno dei due collegamenti veloci per lo scalo aeroportuale. Venne ultimata nel 1968. II secondo collegamento di Roma con il suo principale aeroporto, quello per ferrovia, fu invece realizzato solo nel 1990, in occasione dei mondiali di calcio, ed è stato potenziato con una serie di attrezzature e linee secondarie solo negli anni più recenti grazie alla cosiddetta "cura del ferro" cui si è sottoposta la capitale. 


\section{L'URBANIZZAZIONE E LA NASCITA DELLA CITTÀ METROPOLITANA}

L'urbanizzazione nella campagna romana comincia, dunque, attraverso la localizzazione in punti più o meno strategici delle stazioni di posta $e$, in un periodo successivo, delle stazioni ferroviarie, anche se queste, come è stato sottolineato, erano spesso mal collocate rispetto ai pochi insediamenti esistenti (Tivoli, i Castelli, ...).

Le prime stazioni ferroviarie erano infatti - è utile ricordarlo collocate o a stretto contatto con Roma, che ben presto le inglobò nelle sue prime espansioni fuori le mura, 0 in aree malariche, invivibili in alcune stagioni dell'anno fino al momento in cui si completò la bonifica idraulica; oppure

a una distanza dai nuclei urbani esistenti o troppo limitata perché intorno alla stazione si formasse un nucleo autonomo, o troppo grande per giovare allo sviluppo del piccolo centro preesistente. ${ }^{32}$

Altri poli attrattivi sono state le delegazioni di pubblica sicurezza che, alla fine del secolo, furono ubicate lungo alcune radiali e che il Comune dotò quasi subito dei servizi di base (acqua corrente, servizi igienici, scuole).

Un po' più distante dalle mura sorgeva, poi, l'anello dei forti e delle batterie che, come è stato detto, distavano circa $3 \circ 4 \mathrm{~km}$ dalla città murata: in breve tempo alcuni forti - i primi furono il forte Boccea e quello di Pietralata - funzionarono da richiamo per la popolazione e, in qualche modo, da incentivo per la formazione di nuovi insediamenti più prossimi alla città.

Infine alcuni dei primi nuclei abitati nella campagna romana si formarono su ex proprietà ecclesiastiche, date in enfiteusi ad

32 L. BORTOLOTTI, op. cit., p.148. 
imprenditori borghesi, oppure sulle grandi proprietà nobiliari espropriate o vendute o semplicemente affittate.

Questi esempi hanno, però, rappresentato solamente l'inizio di un lungo e neanche troppo lento processo di urbanizzazione che ha ben presto investito larghe parti di quel

deserto senza case, senza abitanti e perfino senza alberi che era la campagna di Roma, ${ }^{33}$

e che, per semplificare, si può suddividere in tre "passaggi" principali:

- il periodo a cavallo del 1870,

- il periodo fra le due guerre mondiali,

- il periodo tra la fase post-bellica e i giorni nostri.

II primo passaggio importante nella vicenda si può far coincidere con gli anni a cavallo del 1870, quando cioè Roma divenne capitale d'Italia.

Con lo spostamento della capitale, infatti, si determinò un'enorme richiesta di alloggi, che, fino almeno alla fine degli anni '80, l'Amministrazione comunale non riuscì a gestire, o quanto meno gestì con grosse carenze. In questo periodo inizia dunque la difficile fase durante la quale avviene la trasformazione di

una «cittadina di provincia» di poco meno di 250.000 abitanti, tutta raccolta sulle anse del Tevere, in una Città capitale moderna di circa 700.000 abitanti. ${ }^{34}$

Il primo atto che il trasferimento della Capitale impone è la definizione di un nuovo assetto fisico della città, per rispondere a nuove

\footnotetext{
33 L. BORTOLOTTI, op. cit., p.148.

34 G. CucciA, op. cit., p.15.
} 
esigenze funzionali ed organizzative. La domanda di spazi e strutture per la direzionalità, per i servizi e per le abitazioni è infatti notevole.

A Roma, in poco tempo, ai due terzi della popolazione attiva nel settore terziario ereditato dalla città pontificia, si sommano, provenienti da Firenze e da altri centri, 5.000 nuovi impiegati e altrettante famiglie: la domanda pregressa della Roma di Pio IX di residenze raddoppia con i nuovi arrivi; (...). ${ }^{35}$

A questo si aggiunge il ritmo incessante della conseguente nuova immigrazione, che alla fine del decennio raggiunge una media di circa 8.000 abitanti l'anno.

Lo sviluppo edilizio comincia, però, in modo tumultuoso ed incontrollato, in una città che cresce per lo più senza un piano preordinato $^{36}$ che tenga conto di tutte le componenti dell'economia e della vita urbana della città moderna. Gli interventi vengono ideati e realizzati non dal Comune, bensì dagli operatori economici (proprietari, società finanziarie ed imprese di costruzione) che, sollecitati dalle esigenze del mercato e spinti prevalentemente da i propri interessi, iniziano ad operare nelle aree di cui dispongono o che acquisiscono, instaurando rapporti con l'Amministrazione comunale mediante convenzioni, oppure anche senza alcun tipo di permesso. Dal 1871 al 1873 - prima cioè del Piano dell'ing. Viviani - viene stipulata una serie notevole di convenzioni che riguardano ben 160 ettari di aree localizzate nei pressi delle Terme di Diocleziano, del Castro Pretorio, dell'Esquilino e del Viminale prevalentemente per le strutture direzionali e servizi, e a Testaccio, Trastevere, Gianicolo e Prati di Castello per l'espansione edilizia di tipo abitativo.

35 G. CuccIA, op. cit., p.17.

36 Sulla pianificazione comunale e sui Piani Regolatori Generali adottati e approvati dall'inizio del '900 ad oggi, si possono vedere le schede allegate al presente capitolo nelle quale vengono sommariamente descritte le linee di sviluppo indicate da ciascuno strumento urbanistico soprattutto per ciò che riguarda le politiche di piano sull'urbanizzazione che ha via via interessato la campagna romana.

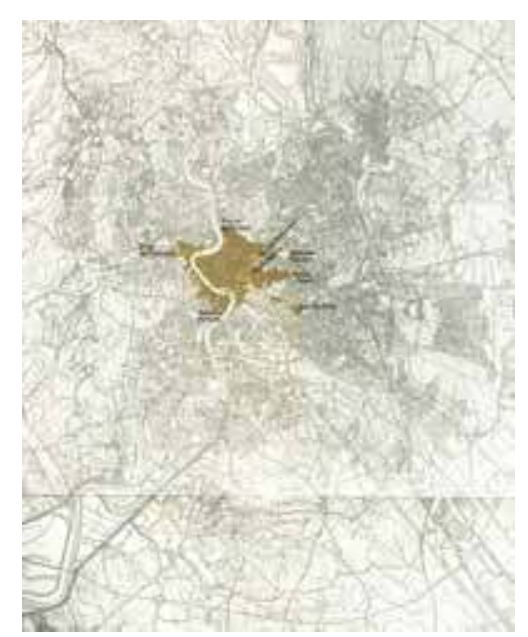

Figura 9. Roma nel 1870: la capitale pontificia occupava principalmente l'ansa del Tevere. Al di là del fiume, le due antiche propaggini erano Borgo e Trastevere. II primo ampliamento della città ottocentesca avviene soprattutto verso la stazione ferroviaria e i colli ad est. 


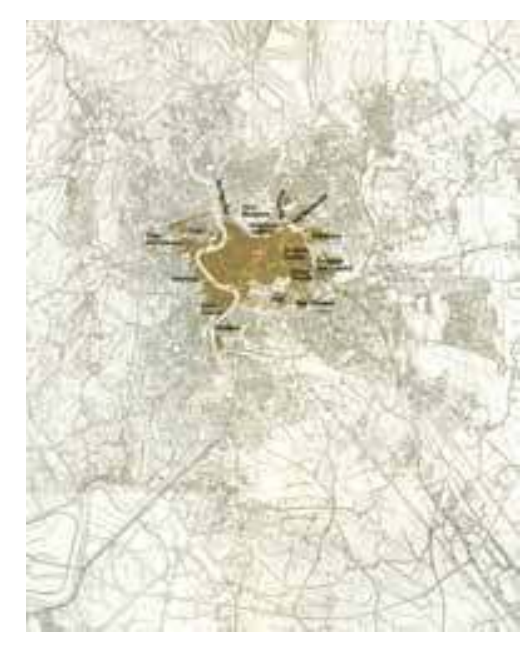

Figura 10. Roma nel 1900: le aree nella direzione della stazione sono ormai sature e nuovi quartieri stanno sorgendo lungo la Flaminia, la Salaria, la Nomentana, San Lorenzo, San Giovanni e Testaccio. Al di là del Tevere è in corso la costruzione di Prati di Castello.
Il problema principale di questa prima fase di espansione è l'integrazione e la continuità delle nuove edificazioni rispetto alla città storica: in sostanza avviene che la città capitale si sovrappone ed incorpora l'assetto della Roma papale, grazie soprattutto al grado di incompletezza che presenta la sua struttura urbana.

Nello stesso tempo, nell'Agro romano si cominciano a realizzare le prime borgate rurali, ovvero dei centri abitati pianificati con il fine di realizzare una colonizzazione agricola del territorio intorno alla città.

La prima realizzazione comunale fu la costruzione di una borgata rurale sulla via Ostiense, anche se subito fu evidente come l'obiettivo fosse quello di realizzare qualcosa di diverso da insediamenti puramente rurali. Furono infatti progettati dei veri e propri centri di servizio, dotati di scuola, stazione sanitaria, caserma dei carabinieri, posta, telefono, con le residenze articolate intorno ad una piazzetta.

Nel 1911 erano già stati presentati 7 progetti di borgate rurali: due vennero immediatamente approvati, tre furono sottoposti a modifiche e due respinti. Nacquero, dunque, come borgate rurali quella della Magliana nel 1911, quella della Bufalotta sulla via delle Vigne Nuove e quella a Monti S. Paolo nel 1913, quella Martini-Marescotti lungo I'Appia nel 1914. 


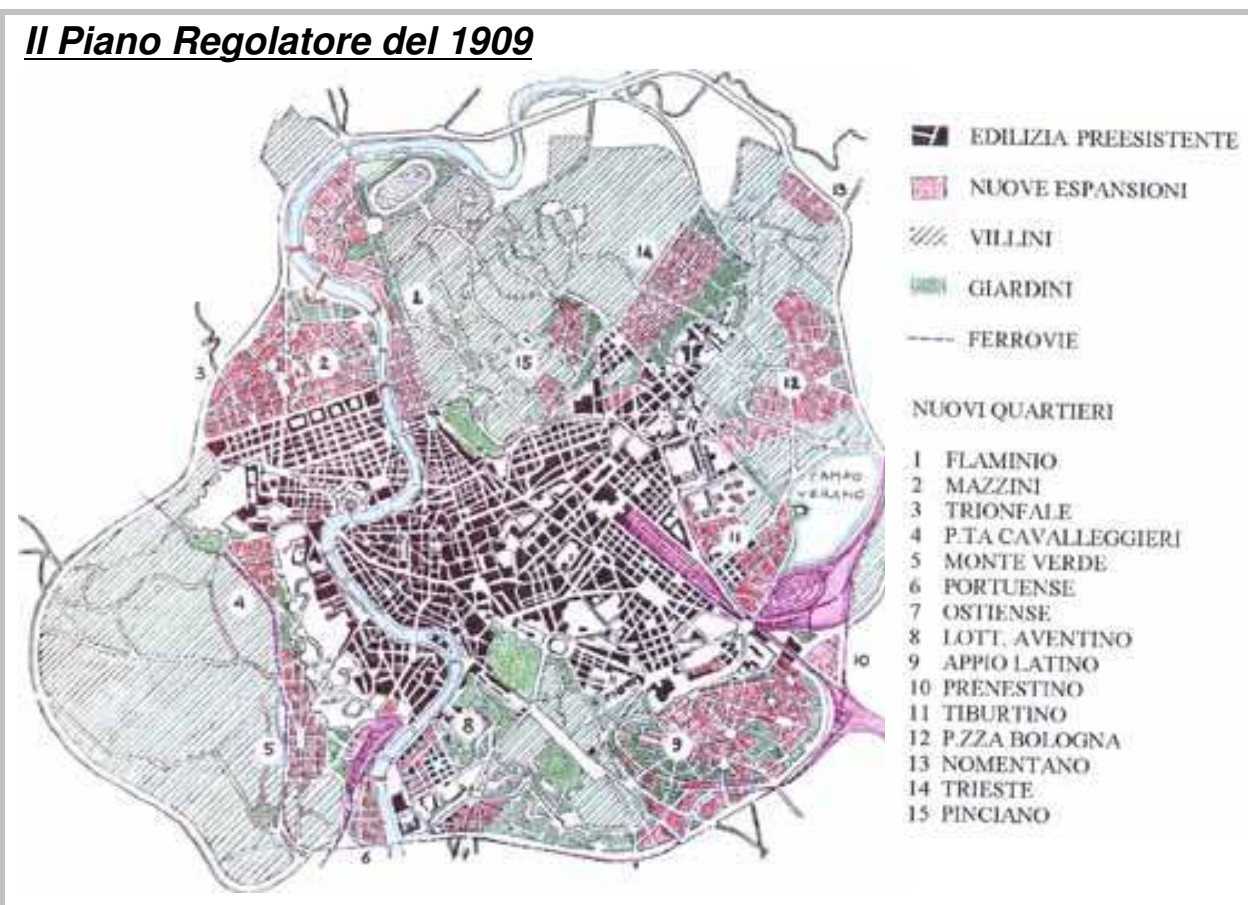

Figura 11. La zonizzazione del Piano Regolatore dell'ing. Edmondo Sanjust di Teulada elaborato nel 1909.

Curato dall'Ing. Edmondo SANJUST DI TEULADA, il piano prevede che vengano realizzati intorno alla città esistente nuovi quartieri per i quali si regolamentano anche le tipologie edilizie: i «fabbricati», ovvero una tipologia intensiva, che possono avere un'altezza massima di 24 metri ed un distacco tra loro pari a 2/3 dell'altezza; $i$ «villini», che devono essere di tre piani e circondati da ogni parte da giardinetti; i «giardini signorili» che possono essere costruiti solo per $1 / 20$ della superficie del lotto e sono caratterizzati da costruzioni di lusso.

La caratteristica principale del piano è dovuta al fatto che il SANJUST, in buona sostanza, vede la città come una successione di quartieri: i provvedimenti per le singole zone, infatti, appaiono decisamente più studiati e convincenti del disegno d'insieme di tutto il PRG.

L'uso delle diverse tipologie edilizie è evidentemente il mezzo attraverso cui il Comune cerca di evitare il fenomeno "macchia d'olio", ossia cerca di impedire l'indiscriminato dilagare delle abitazioni in tutte le direzioni mediante l'alternanza di zone ad alta densità con altre con densità più bassa, dunque meno abitate, e con tipologie residenziali di maggior lusso. 
II secondo passaggio dello sviluppo della città comincia subito dopo la fine della prima guerra mondiale e finisce, in sostanza, alla vigilia del secondo conflitto mondiale, attraversando quindi tutto il ventennio fascista.

Con la prima guerra mondiale, i problemi che già erano sorti con Roma capitale si aggravano ulteriormente, in maniera disastrosa: per tutti gli anni del conflitto, infatti, non si costruì nulla, soprattutto a seguito della mancanza di manodopera, di capitali e di materiali e, nello stesso tempo, la popolazione della città aumentò per il richiamo esercitato dalle industrie belliche e per l'afflusso di profughi che provenivano dalle zone maggiormente colpite dalla guerra.

Nel periodo fra le due guerre i fenomeni dell'immigrazione, anziché rallentare, in realtà accelerano e lo Stato emana alcuni provvedimenti attraverso i quali fronteggiare in qualche maniera la crisi edilizia, l'occupazione abusiva degli alloggi e la disoccupazione.

Questi provvedimenti, insieme con l'abusivismo ormai definito «di necessità» e con le leggi particolari per Roma che legalizzavano lo stravolgimento del piano regolatore del 1909, sono alle radici del disordine urbanistico della capitale. ${ }^{37}$

Un esempio di quanto afferma Lando Bortolotti è il Testo Unico sull'edilizia economica e popolare emanato nel 1919, che prevedeva delle facilitazioni per Roma autorizzando I'Unione edilizia nazionale, I'Istituto case popolari e l'Istituto cooperativo a costruire, nella città e nel territorio intorno ad essa, case economiche anche per conto di cooperative e di privati. Considerando, dunque, le costruzioni come urgenti e indifferibili, una volta che fosse approvato il progetto, i terreni occorrenti potevano essere occupati senza alcuna dilazione e l'indennità da pagare ai proprietari di quest'ultimi era quella della Legge Napoli del 1885 per le zone interne alle previsioni di piano regolatore, mentre per le

37 L. BORTOLOTTI, op. cit., p.156. 
aree al di fuori del piano l'indennità era commisurata al valore agricolo del terreno. II risultato fu che privati e cooperative collocarono quelle che ostentatamente chiamavano “città giardino» un po' dappertutto ${ }^{38}$, anche in aree molto lontane dal centro urbano: esse divennero, nella maggior parte dei casi, i nuclei di future espansioni.

II periodo è dunque segnato dalla prima grande espansione urbana ed il quadro politico è determinato in larga misura dall'azione del Governatorato fascista, che, di fatto, si sostituisce completamente agli organi municipali.

È sulla base delle esaltazioni del mito della romanità che si procede nel centro storico a tutta una serie di sventramenti che vengono caldeggiati anche dalla cultura tecnica ufficiale del tempo per due ordini di motivi:

la modernizzazione della città in rapporto alle esigenze della mobilità e la rappresentazione del regime attraverso nuove sistemazioni simboliche e monumentali. ${ }^{39}$

Partendo da queste motivazioni vengono, dunque, giustificati sia le alterazioni irreversibili che avvengono nel tessuto storico del centro urbano, sia l'allontanamento d'autorità dei ceti meno abbienti, che vengono di fatto spostati in aree periferiche e mal servite.

Il fenomeno della borgata, come luogo di «deportazione» dei ceti popolari espulsi dal centro in seguito alle demolizioni, diventa macro-scopico sotto il fascismo. ${ }^{40}$

38 A onore del vero il Comune tentò di porre riparo agli effetti del Testo Unico, cercando di incanalare in direzioni opportune le pressioni delle cooperative e dei privati che aspiravano ad un'abitazione ed identificò 14 zone già investite dall'urbanizzazione entro le quali si poteva applicare quanto previsto dalla legge nazionale. Di queste aree - che però l'Amministrazione comunale non riuscì ad imporre - sette erano entro i limiti del piano regolatore vigente e altrettante erano quelle esterne.

39

40 M. SANFILIPPO, Le tre città di Roma. Lo sviluppo urbano dalle origini ad oggi, Laterza, Roma-Bari 1993, p.350-351.

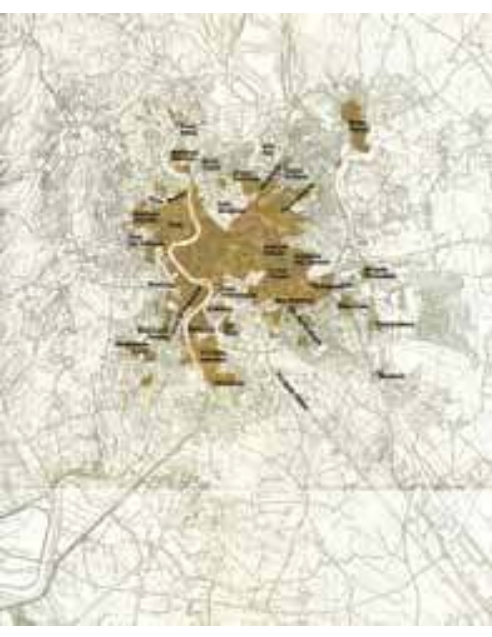

Figura 12. Roma nel 1930: alla fine del 1930 l'espansione della città interessa ormai tutte le direzioni, con i quartieri borghesi verso nord-est e quelli popolari verso est e sud. 
Nel 1924 compare la prima borgata rurale ufficiale, Acilia, costruita sulla strada che "porta Roma al mare", risanando una zona malarica. In questo nuovo nucleo insediativo vengono presto trasferiti molti abitanti dell'antico quartiere dei Fori, che nei primi anni '30 il fascismo sventra per riportare alla luce i fasti dell'antica Roma.

Alla fine degli anni '20 sono, quindi, velocemente progettate e costruite le borgate di S. Basilio, Prenestina, Gordiani, Primavalle, dove la tipologia edilizia prevalente è quella della baracca, priva di servizi igienici e acqua corrente. Successivamente vengono realizzate le borgate dove andranno a vivere i nuovi immigrati, gli abitanti allontanati dai vecchi rioni colpiti dagli sventramenti e quelli dei vecchi borghetti, nelle quali, al contrario delle precedenti, la tipologia edilizia prevalente è quella dei cosiddetti "dormitori pubblici" dotati sì di servizi igienici, ma di qualità scadente.

A questo tipo di "edificazione legale" si aggiunge negli stessi anni l'edificazione abusiva, che viene subito definita come «abusivismo di necessità», in quanto l'immigrato proveniente dalle campagne, un po' perché non ha i mezzi sufficienti per pagare l'affitto di una casa, un po' seguendo la tendenza abituale delle popolazioni rurali, comincia a costruirsi con le proprie mani la propria abitazione.

Un editorialista de «ll Mondo» nell'agosto del 1924 scriveva:

Nelle località in cui pochi mesi o pochi giorni or sono era ancora la campagna nuda, si scorgono aggruppate numerose casette, alcune costruite con materiale in muratura, altre costruite in parte in muratura e in parte con altro materiale, alcune in legno costruite a regola d'arte e con qualche dispendio, altre messe insieme rozzamente con tavole in legno mal connesse, altre ancora di fango, di pezzi di legno e di pezzi di latta, tutte con orientamenti disordinati. ${ }^{41}$

La campagna romana si riempie, dunque, di borgate e di nuclei più 0 meno edificati e più o meno legalizzati, alcuni dei quali, ben presto,

41 Citazione desunta da L. BORTOLOTTI, op. cit., p.159. 
vengono chiamati dalla stessa Amministrazione fascista «villaggi abissini», per via della loro stretta somiglianza con i villaggi di capanne etiopi. Sono agglomerati di baracche, molto spesso lontani da infrastrutture e servizi di supporto, costruiti - come abbiamo visto - con materiali di risulta e di scarto e con rifornimenti idrici e servizi igienici di fortuna.

Negli anni successivi in questi nuclei si assiste pian piano alla progressiva sostituzione delle primitive baracche con costruzioni più solide, specialmente dopo l'arrivo delle strade, dell'acqua, del sistema fognario.

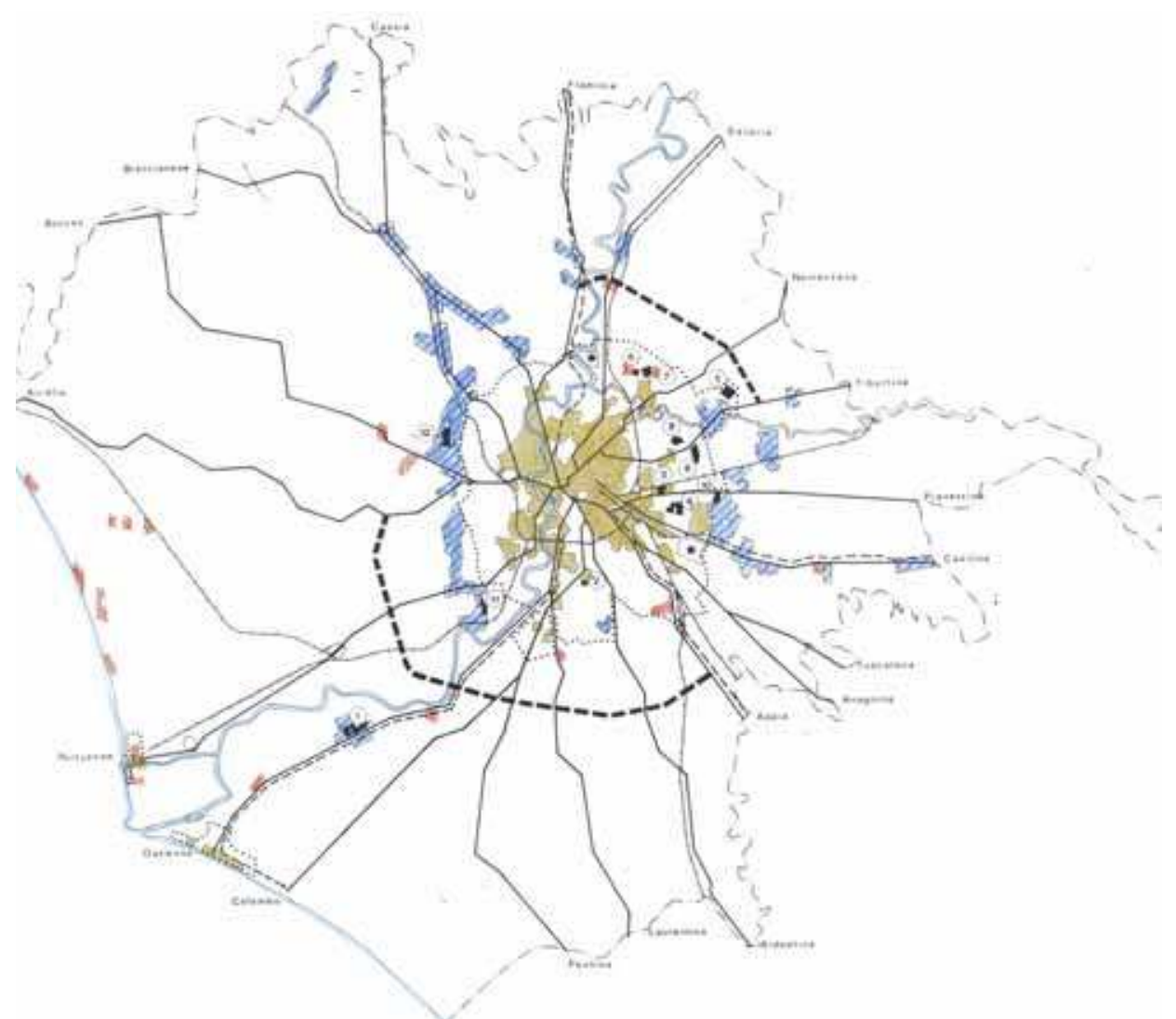

Figura 13. La situazione delle borgate - ufficiali e abusive - nel 1935: sono riportati in rosso gli insediamenti abusivi, in blu i nuclei edilizi perimetri nel 1935, in nero le borgate di iniziativa pubblica. 
Perimetrati nel 1935 e quindi regolamentati rispetto al piano regolatore dallo stesso Governatorato fascista, diventeranno precursori dell'abusivismo, dilagante soprattutto nel periodo del boom economico del dopoguerra.

Alla data dell'approvazione del piano del Governatorato nel 1931, nella parte di territorio comunale esterna all'area urbana che, in quanto tale, non viene sottoposta a pianificazione e che corrisponde in larga parte all'Agro romano, convivevano dunque due diversi tipi di urbanizzazione: l'urbanizzazione legale e l'urbanizzazione abusiva.

All'interno del primo tipo - l'urbanizzazione legale - si possono riconoscere situazioni che mutano i propri caratteri a seconda delle diverse connotazioni dei soggetti ai quali l'intervento è destinato:

- gli alloggiamenti precari e temporanei per baraccati e sfrattati, eseguiti dal Governatorato, localizzati in aperta campagna in luoghi non visibili dalle grandi arterie stradali, costituiti da baracche e/o da casette «rapide» e «rapidissime»;

- gli interventi, anch'essi pubblici, di edilizia popolare che hanno dato origine in un primo periodo - tra il 1925 ed il 1930 - alla città giardino della Garbatella e in un secondo periodo - tra il 1931 ed il 1935 - ai grandi contenitori intensivi localizzati alle estreme propaggini della città (Monte Sacro, Val Melaina, ecc...). Un terzo ed ultimo periodo compreso tra il 1936 ed il 1942 - vede la realizzazione di alcune delle cosiddette "borgate operaie semirurali", fuori dai collegamenti con il centro, completamente prive di connessioni con i luoghi del sistema produttivo ai quali sembrava dovessero essere affiancate;

- le lottizzazioni private convenzionate, eseguite il più delle volte da piccole imprese artigiane, che danno origine, in aree periferiche rispetto al centro urbano, a situazioni di compromesso fra la borgata agricola, con la casetta unifamiliare e l'orticello, e il villino di lusso delle lottizzazioni urbane di media densità.

Entro l'urbanizzazione abusiva, invece, vi sono due situazioni tipo: 
- quella delle baracche o "villaggi abissini", che per un certo periodo il regime tenta di annullare spostando i baraccati nelle cosiddette borgate ufficiali;

- quella delle lottizzazioni edificate da piccoli proprietari, che si vanno solitamente a collocare in prossimità delle vie consolari ${ }^{42}$ e delle lottizzazioni realizzate nei periodi precedenti e danno origine ad una serie di nuclei distribuiti omogeneamente ai margini della città legale. Alcune delle situazioni fino ad ora descritte tendono a sovrapporsi quando, nel 1935, il governatore di Roma Bottai inventa un marchingegno attraverso il quale si trova l'escamotage di costruire quasi ovunque fuori dal piano, facendo così diventare "regolari" la gran parte delle lottizzazioni private. Vengono infatti identificati e perimetrati un buon numero di «nuclei edilizi», realizzati antecedentemente all'approvazione del piano, che vengono di fatto regolarizzati. Grazie a ciò, si definirono come già urbanizzati e suscettibili di ulteriori edificazioni ben $29 \mathrm{~km}^{2}$ di Agro romano e si aumentarono di circa il $20 \%$ le previsioni di espansione contenute nel già vecchio piano regolatore.

42 Rappresentano un esempio il Quadraro, Torpignattara e Centocelle ad est lungo la via Casilina, oppure Tor di Quinto, a nord lungo la Cassia. 


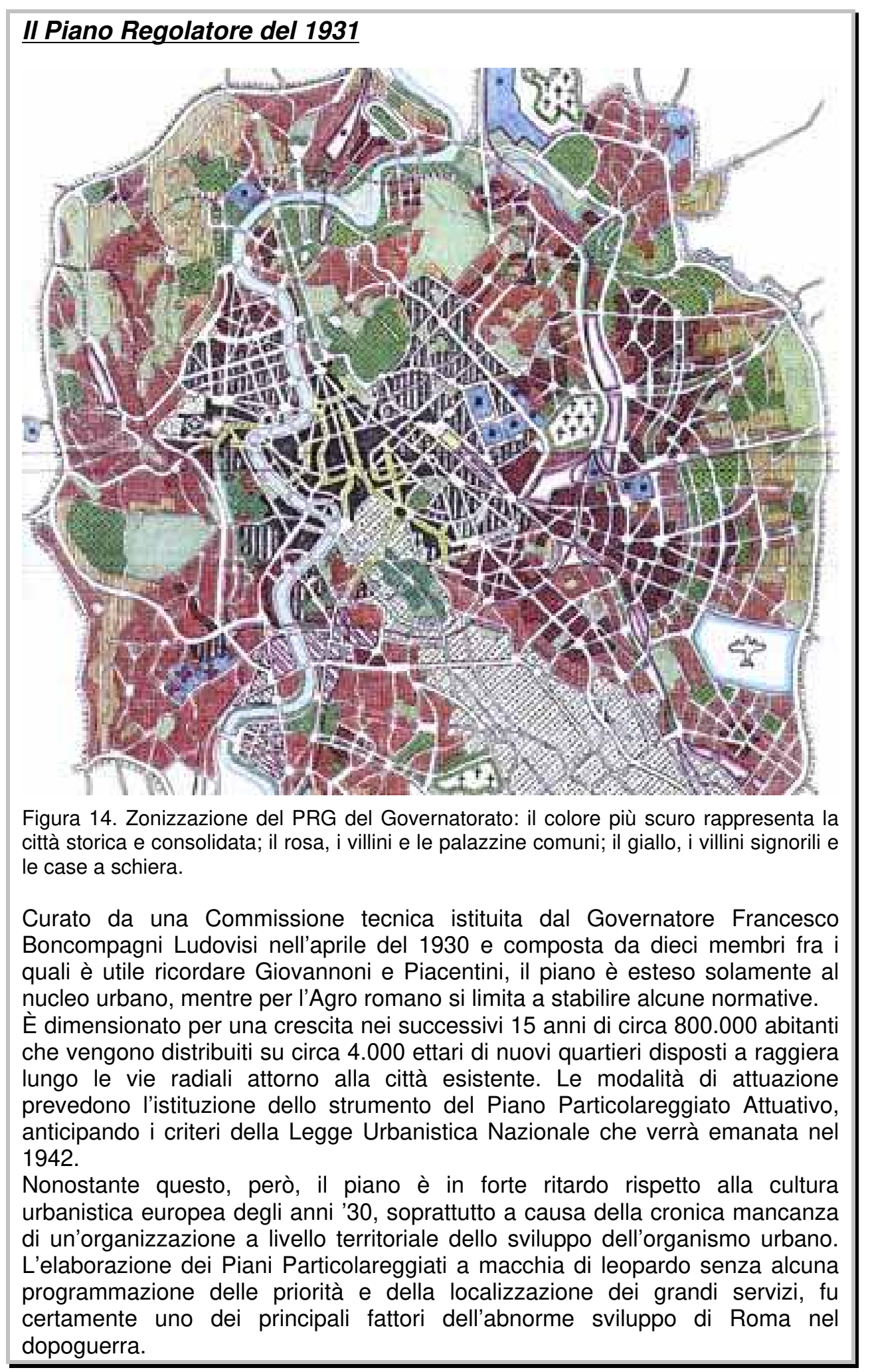


II terzo ed ultimo passaggio dello sviluppo della città comincia subito dopo la fine della seconda guerra mondiale e si conclude ai giorni nostri.

Roma si sveglia dall'incubo della guerra senza aver subìto estese devastazioni dai bombardamenti degli alleati 0 dai vandalismi dell'esercito tedesco in ritirata. Nonostante tutto si presentano comunque dolorosi aspetti di disordine e di miseria, tanto in città (scuole occupate dalla gente rimasta senza casa, strade dissestate, trasporti pubblici improvvisati), quanto soprattutto in campagna, dove una gran massa di sfollati provenienti dai paesi dell'Italia del centro-sud si sono accampati in condizioni di massimo disagio. A tutto ciò si associa il fenomeno dell'immigrazione che riprende pieno vigore e fa sì che la città ricominci ad espandersi a macchia d'olio in ogni direzione, caratterizzata da un'edilizia di tipo espansivo e per lo più incontrollato, senza un disegno organico alle spalle.

Superato il periodo immediatamente successivo alla fine della guerra, l'attività edilizia infatti riprende a pieno ritmo e il piano regolatore del 1931 viene messo in atto con tutte le conseguenze negative dovute al modello di intervento urbanistico previsto con i Piani Particolareggiati. La loro redazione è infatti fortemente condizionata dalla proprietà fondiaria che, mirando soprattutto a far crescere i propri utili, attua un innalzamento delle densità abitative, una sostituzione dei tipi edilizi e una riduzione degli spazi pubblici. L'intervento è pressoché ovunque privato, con un meccanismo attuativo che tende a scaricare sul Comune i costi delle opere di urbanizzazione che, in molti casi, sono in ritardo rispetto a quelle delle costruzioni vere e proprie.

Continua in parallelo, e in maniera ancora più forte ed invasiva del periodo ante-guerra, l'espansione incontrollata degli alloggi precari di ogni tipo, tanto che ancora nel 1958 nell'area del Comune di Roma ne sono ufficialmente censiti oltre 13.000 tra grotte, ruderi, baracche, seminterrati, scantinati, ecc. II fenomeno dell'abusivismo, però, comincia 


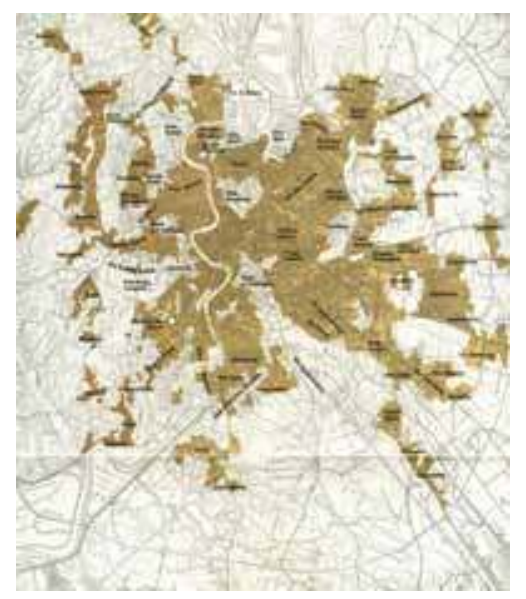

Figura 15. Roma nel 1960: la dimensione della città è ormai enorme e l'espansione avviene in tutti i settori, tendendo anzi a saturare quelli meno interessati nei decenni precedenti. Oltre i limiti riportati su questa planimetria, Roma tende a saldarsi a sud alla massiccia espansione di Ostia e delle borgate disseminate tra la città e il mare. a perdere i caratteri di marginalità assoluta che lo avevano contraddistinto fino a quel momento e si configura come un vero e proprio modo di produzione alternativo della casa.

II piano del 1931 lasciava sostanzialmente scoperti - non regolamentati - circa 130.000 ettari che furono, in un arco di tempo che va dal 1949 al 1962, esposti alle forti speculazione della proprietà fondiaria, la quale diede avvio a numerosi frazionamenti, il più delle volte illegali.

Un ruolo determinante nella localizzazione di buona parte degli insediamenti abusivi fu svolto da quei «nuclei edilizi» perimetrati nel 1935 intorno ai quali si andò a concentrare la maggior parte delle nuove lottizzazioni.

Lungo la via Casilina, Giardinetti e Finocchio incentivano la crescita di Torre Angela e Borghesiana; lungo la via Trionfale nasce e si consolida la borgata Ottavia; lungo la via Boccea le borgate di Casalotti e Montespaccato e così via fino al litorale, dove cominciano a comparire le prime abitazioni abusive a Focene, Isola Sacra e Passoscuro. ${ }^{43}$

Fino al 1960 vengono urbanizzati oltre 4.000 ettari di territorio agricolo, producendo un ampliamento complessivo del territorio urbano di circa l'80\%. Ciò che in primo luogo causa una crescita così consistente è la componente abusiva, che raggiunge dimensioni notevoli e, per i suoi caratteri di insediamento povero e a basso costo, è testimone della larga esclusione dei ceti sociali marginali dalle politiche abitative messe in atto e gestite dalle istituzioni.

Oltre agli imponenti quartieri di edilizia agevolata (il Tuscolano ed il Tiburtino ne sono alcuni esempi) prodotti dall'Ina casa e dal Comune, si consolidano nel territorio dell'agro romano diverse situazioni urbanizzative significative:

\footnotetext{
43 M. Olivieri, 1925 - 1981: la città abusiva, in A. Clementi, F. Perego (a cura di) «La metropoli "spontanea". Il caso di Roma», Dedalo, Roma 1983, p.293.
} 
- le lottizzazioni abusive, dove il proprietario dell'area - direttamente o attraverso alcuni intermediari - provvede al frazionamento del terreno in lotti che hanno una dimensione solitamente compresa tra $\mathrm{i}$ 500 ed i 1.000 mq, traccia i percorsi carrabili di supporto e promuove la vendita delle aree ${ }^{44}$

- le borgate marine, che originariamente sorgono sul litorale non come agglomerati di abitazioni saltuarie, bensì come veri e propri piccoli nuclei abitati dai lavoratori occupati nelle aziende vicine o dai pescatori $^{45}$;

- le borgate rurali, formate o da cooperative di agricoltori che acquistano i terreni per un'utilizzazione a scopi produttivi e che ricorrono all'aiuto di mutui agevolati concessi dallo Stato per incentivare l'agricoltura, o da cooperative di ex combattenti cui vengono distribuite in uso aree di proprietà pubblica sempre con scopi agricoli ${ }^{46}$.

Con il Piano Regolatore Generale del 1962 il meccanismo dell'espansione incontrollata della città nella campagna romana non solo non si arresta, ma si accentua e assume sempre più decisamente un carattere di "abusivismo edilizio" in contrasto con i programmi urbanistici comunali.

II nuovo strumento urbanistico - così come era già avvenuto per il precedente - interviene a posteriori rispetto alla situazione illegale che abbiamo visto essersi formata antecedentemente e attraverso la

44 Secondo questo meccanismo sorgono o si ampliano: ad est Torre Angela, Finocchio, Borghesiana, Giardinetti, Torre Maura, Tor Sapienza, La Rustica, Lunghezza; a sud Quarto Miglio, Casal Morena, Castel di Leva; ad ovest Boccea, Casalotti, Pisana, Montespaccato, Magliana; a nord Castel Giubileo, Labaro, Cesano.

45 Nascono in questo modo Fiumicino - Isola Sacra, Focene, il Villaggio dei Pescatori, Passoscuro.

46 Sorgono con il primo meccanismo Castelverde e Porta Medaglia, con il secondo Gregna - S.Andrea, Romanina, Passo Lombardo. 
normativa per le zone $F_{1}$ riconosce non solo formalmente i nuclei abusivi sparsi nel territorio dell'agro.

Queste zone ricoprono una superficie di circa 3.800 ettari e si caratterizzano diversamente a seconda della parte di città in cui sono sorte: ad est, infatti, ci si trova di fronte a più nuclei sorti lungo le consolari radiali in continuità con il tessuto edilizio precedente; ad ovest, invece, sono grossi nuclei disposti in maniera tangenziale rispetto al corpo della città e sono il più delle volte nettamente staccati dal tessuto urbano preesistente; verso il mare e a nord, infine, le zone $F_{1}$ mantengono un carattere frammentario e sporadico, mentre mancano quasi del tutto in direzione sud.

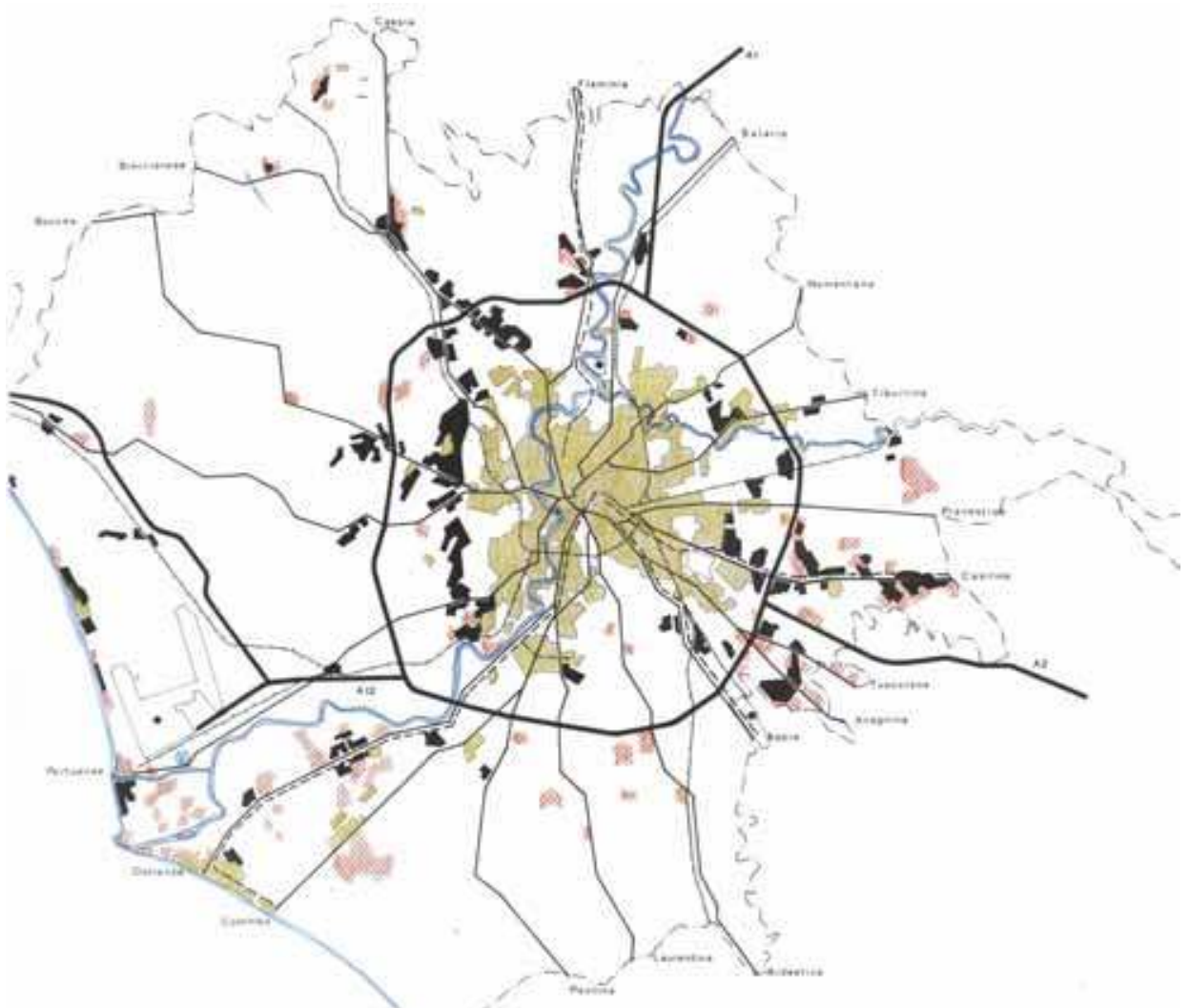

Figura 16. La situazione dei nuclei abusivi e delle zone $F_{1}$ nel 1971: sono riportati in rosso gli insediamenti abusivi ed in nero le zone $F_{1}$ perimetrate dal PRG del 1962. 
In questo periodo si assiste dunque ad uno sviluppo senza precedenti dell'abusivismo e ad una sua affermazione come modo ordinario di produzione edilizia. Nascono

contemporaneamente i primi segnali di una modificazione strutturale dei processi che [...] vedranno affiancarsi alla figura degli autocostruttori, motivati dall'intervento dello stato di necessità, gli autopromotori che operano prevalentemente per convenienza (anche se permane l'uso diretto dell'abitazione) ed i piccoli, per il momento, promotori immobiliari. ${ }^{47}$

Si assiste ad una veloce ed inesorabile trasformazione della produzione edilizia illegale, che passa da una forma di carattere individuale, determinata quasi sempre da uno stato di necessità, ad una fase basata essenzialmente su una logica speculativa, che dà origine ad un vero e proprio mercato edilizio e della compravendita delle aree parallelo a quello legale dell'amministrazione comunale. Al dilagare delle lottizzazioni andrà sempre più a corrispondere un intervento che realizzerà ville di lusso, attività commerciali, palazzine destinate alla vendita o all'affitto.

L'urbanizzazione illegale tende a spostarsi dalle zone $F_{1}$, dove il prezzo dei terreni è aumentato vertiginosamente, e ad occupare ulteriormente ciò che resta della campagna romana. Va dunque a localizzarsi soprattutto nelle aree poste agli immediati margini delle vecchie lottizzazioni ex abusive, $\mathrm{o}$ in zone ancora più esterne, o ancora vicino alle borgate rurali che in questo modo assumono la conformazione di veri e propri nuclei urbani.

La sua dilatazione nel territorio non avviene per insiemi di zone definite da destinazioni derivanti dalla realizzazione di un preciso programma economico od urbanistico, ma secondo un incontrollato disporsi di «onde edilizie» con caratteristiche prevalentemente residenziali, in alcuni casi miste ad opifici e

\footnotetext{
$47 \quad$ M. OlIVIERI, op. cit., p.297.
} 
depositi, ma comunque carenti di servizi e di qualsiasi tipo di organizzazione urbana. $^{48}$

I maggiori processi di concentrazione e di proliferazione si sviluppano ancora nel settore est, lungo la Prenestina, la Casilina, la Tuscolana e l'Anagnina, fino a toccare il confine comunale e congiungersi con gli insediamenti edilizi dei Comuni limitrofi.

Nella direzione mare, il comprensorio tra l'autostrada di Fiumicino e la via Cristoforo Colombo tende a saturarsi con la formazione di lottizzazioni a bassa densità che interessano vaste porzioni della campagna romana della bonifica a Isola Sacra, Stagni di Ostia, Infernetto. Lungo la costa gli incrementi dell'urbanizzazione avvengono per espansioni contigue ai nuclei formatisi precedentemente a Focene, Fregene, Villaggio dei Pescatori, Passoscuro. Nel settore ad ovest della città, poi, gli insediamenti mantengono una disposizione per grossi agglomerati tangenti il GRA, mentre a nord e a sud i nuclei si collocano isolatamente sul territorio.

Appartiene a questo periodo l'inizio di un nuovo processo di urbanizzazione abusiva che si affianca a quello tipicamente residenziale. Si comincia infatti a formare tutta una serie di aree con funzioni di tipo commerciale ed artigianale - produttivo a volte miste alle residenze, a volte organizzate in consorzi autonomi, a volte isolate, che si localizzano soprattutto in quelle porzioni di campagna romana che per il minor costo delle aree e la facilità dei collegamenti facilitano l'operazione economica.

Determinante per questo tipo di operazioni è stata l'assenza di una programmazione dello sviluppo produttivo della città, legata alla logica di una precisa scelta politica che risale già ai tempi dell'unificazione nazionale, tutta tesa a caratterizzare la capitale quale centro burocratico

48 P. VISENTINI, La formazione dell'abusivismo nel territorio romano, in A.M. LEONE (a cura di), «ll recupero degli insediamenti abusivi», USPR - Documenti 1 (pubblicazione a cura del Comune di Roma), Roma 1981, p.10. 
e terziario del paese. Questa scarsità di aree unitamente agli elevati prezzi del mercato ha di fatto provocato, da una parte, un accumulo di domande di insediamento produttivo rimaste insoddisfatte e, dall'altra, il diffondersi incontrollato ed indiscriminato, su buona parte del territorio comunale, di costruzioni abusive destinate ad attività di tipo artigianale $\mathrm{e}$ produttivo in un primo tempo, commerciale e direzionale in tempi più recenti.

La grande maggioranza degli insediamenti abusivi è dunque sorta lungo il Grande Raccordo Anulare - all'interno della fascia di rispetto di questo - stringendo la città costruita in più parti e creando volumi di traffico e fenomeni di pendolarismo inaspettati.

Alla fine degli anni ' 70 e primi anni ' 80 , mentre, come abbiamo visto, nell'abusivismo si fanno sempre meno riconoscibili gli elementi strutturali storici che lo hanno caratterizzato, prende forma l'intervento straordinario dell'amministrazione comunale che, con una variante al P.R.G. del 1962 finalizzata specificatamente al «recupero dei nuclei edilizi sorti in difformità dalle indicazioni di piano regolatore», introduce nella normativa tecnica le cosiddette "zone O".

Dal punto di vista quantitativo la variante ha interessato 83 nuclei per una superficie totale di circa 4.550 ettari abusivi realizzati tra il 1962 ed il 1978, in gran parte ad originaria destinazione agricola: di questa superficie, 3.677 ettari,

corrispondenti alle aree abusivamente edificate, hanno assunto una nuova destinazione a carattere prevalentemente residenziale (...); i restanti 763 ettari sono stati invece vincolati per soddisfare il fabbisogno di aree pubbliche nei singoli nuclei (313 ettari per servizi e 450 ettari per verde pubblico). ${ }^{49}$

49 A.M. LEONE, II recupero urbanistico dei nuclei edilizi abusivi, in A.M. LEONE (a cura di), «ll recupero degli insediamenti abusivi», USPR - Documenti 1 (pubblicazione a cura del Comune di Roma), Roma 1981, p.39. 


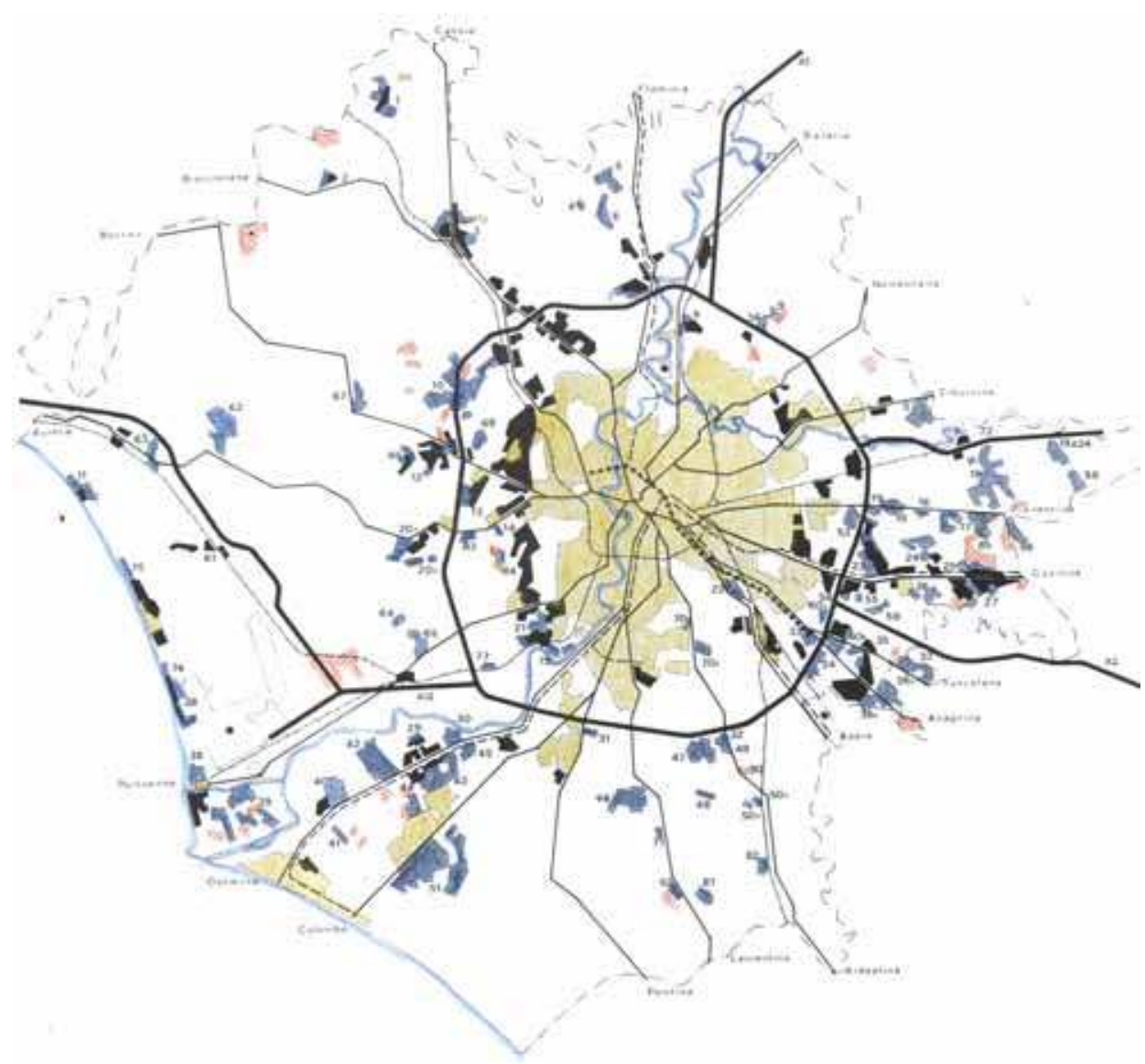

Figura 17. La situazione dell'urbanizzazione con la variante delle zone O: sono riportati in rosso gli insediamenti abusivi, in blu le zone O ed in nero le zone $F_{1}$ del PRG 1962.

La "zona O", a seconda delle diverse situazioni e consistenze urbanizzative sparse sul territorio, nella variante è stata a sua volta suddivisa in tre sottozone:

- le situazioni più consolidate e più densamente costruite, per la maggior parte dei casi localizzate nei settori est (via Casilina e via Prenestina) ed ovest (via Trionfale) della città;

- le situazioni intermedie, meno edificate delle precedenti e di formazione più recente, relative principalmente ai settori sud e sudovest della città; 
- le situazioni da salvaguardare, come la fascia costiera e il parco dell'Appia Antica.

Nel frattempo la produzione edilizia residenziale si assesta su livelli fisiologici, soprattutto grazie alla parallela stabilizzazione demografica, e si assiste sempre meno frequentemente all'occupazione di ciò che resta della campagna romana con nuove urbanizzazioni residenziali, sia regolari che abusive.

Ciò che va avanti e si consolida è, invece, l'urbanizzazione di tipo direzionale, in quanto a metà degli anni Ottanta la città si trova a dover fronteggiare una forte spinta e richiesta di spazi nel settore terziario (con la creazione di circa 90.000 nuovi posti di lavoro), senza riuscire a mettere in moto il Sistema Direzionale Orientale.

In alternativa allo SDO, che rimarrà sempre una grande opera semplicemente annunciata, diventano più appetibili aree già accessibili, dotate di infrastrutture e che sfruttano fattori di localizzazione legati all'area centrale, all'EUR, al Grande Raccordo Anulare lungo il quale come abbiamo già visto - nuclei di terziario industriale sono in via di formazione.

È così che accanto ad un consistente processo di riuso, ristrutturazione e modernizzazione delle sedi esistenti, matura (...) un decentramento periferico che privilegia le aree semicentrali e la direttrice meridionale verso l'EUR, verso i Castelli e verso Fiumicino. ${ }^{50}$

Si strutturano in questo modo i centri direzionali del Parco dei Medici alla Magliana, lungo l'autostrada per Fiumicino, dove si concentrano le sedi di importanti aziende pubbliche e private come l'Alitalia; il nucleo delle Tre Fontane, dove si collocano il Ministero del Tesoro, l'AGIP ed il Pubblico Registro Immobiliare; il Centro Laurentino, con i Ministeri delle Poste e delle Finanze, la SIP e I'IBM; il nucleo Cinecittà - Anagnina, che viene scelto per le sedi decentrate dell'ANAS, ISTAT, INPS e SIP; lungo

50 G. CuccIA, op. cit., p.208. 


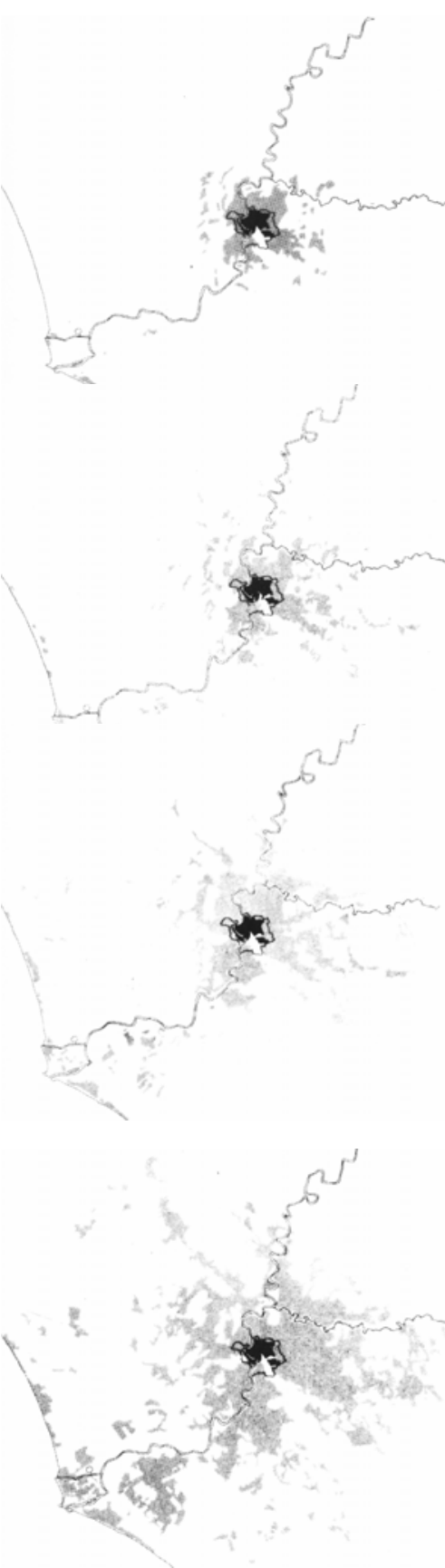

Figure 18-21. La crescita dell'occupazione di suolo per usi urbani nella campagna romana. Dall'alto: 1951, 1964, 1977, 1991. la Tiburtina si insedia il Centro elaborazione dati del Ministero del Tesoro, a Pietralata gli uffici del Ministero dei Trasporti; e così via in altre zone della città.

[...] come si presenta oggi la città? Roma è contemporaneamente vicina alla saturazione e dispersa. II territorio urbanizzato si è quintuplicato negli ultimi 40 anni (da 7.000 a 60.000 ettari). ${ }^{51}$

L'urbanizzazione delle zone abusive, insieme a quelle facenti parte delle espansioni legali, ha occupato migliaia di ettari di campagna romana in maniera irrazionale, informe e frammentaria e ha così disegnato una situazione territoriale che, dividendo il territorio comunale in cinque settori, si può sinteticamente descrivere nei seguenti punti:

\section{- settore sud-ovest}

si è sostanzialmente vicini alla saturazione: a parte il polmone della tenuta di Castel Porziano, Roma è davvero arrivata al mare come voleva Mussolini. Le espansioni più o meno regolari dei centri lungo la costa, unitamente alle zone artigianali ed industriali di più recente formazione, hanno in buona parte alterato le regole storiche della bonifica idraulica andando a sovrapporsi alle maglie e ai sistemi da essa individuati;

\section{- $\quad$ settore sud-est}

le ultime propaggini della periferia abusiva tendono alla saldatura con le aree urbanizzate periferiche dei Comuni della zona dei Castelli Romani, stringendo in una sorta di vero e proprio assedio i confini del Parco dell'Appia Antica, che rappresenta un vero e proprio polmone verde per questa porzione di territorio;

51 F. Ciccone, Roma: capitale senza piano, in G. Campos Venuti, F. Oliva (a cura di) «Cinquant'anni di urbanistica in Italia. 1942 - 1992», Laterza, Roma - Bari 1993, p.252. 
- $\quad$ settore nord-est

anche qui ci si trova di fronte ad una saldatura con l'urbanizzazione dei Comuni contermini, determinata dalla crescita incontrollata di insediamenti prevalentemente residenziali a bassa densità, più 0 meno legali: lembi di campagna romana sono ancora presenti in quest'area lungo il fiume Aniene, caratterizzato lungo le sue sponde dal sistema delle forre (in riva sinistra) e delle ultime tenute (in riva destra);

- $\quad$ settore ovest

la periferia della città, costituita per lo più dai nuclei residenziali abusivi (ormai sanati) e dai grandi centri direzionali localizzati tangenzialmente al Grande Raccordo Anulare, sta erodendo le ultime porzioni di campagna romana rimaste miracolosamente intatte. 


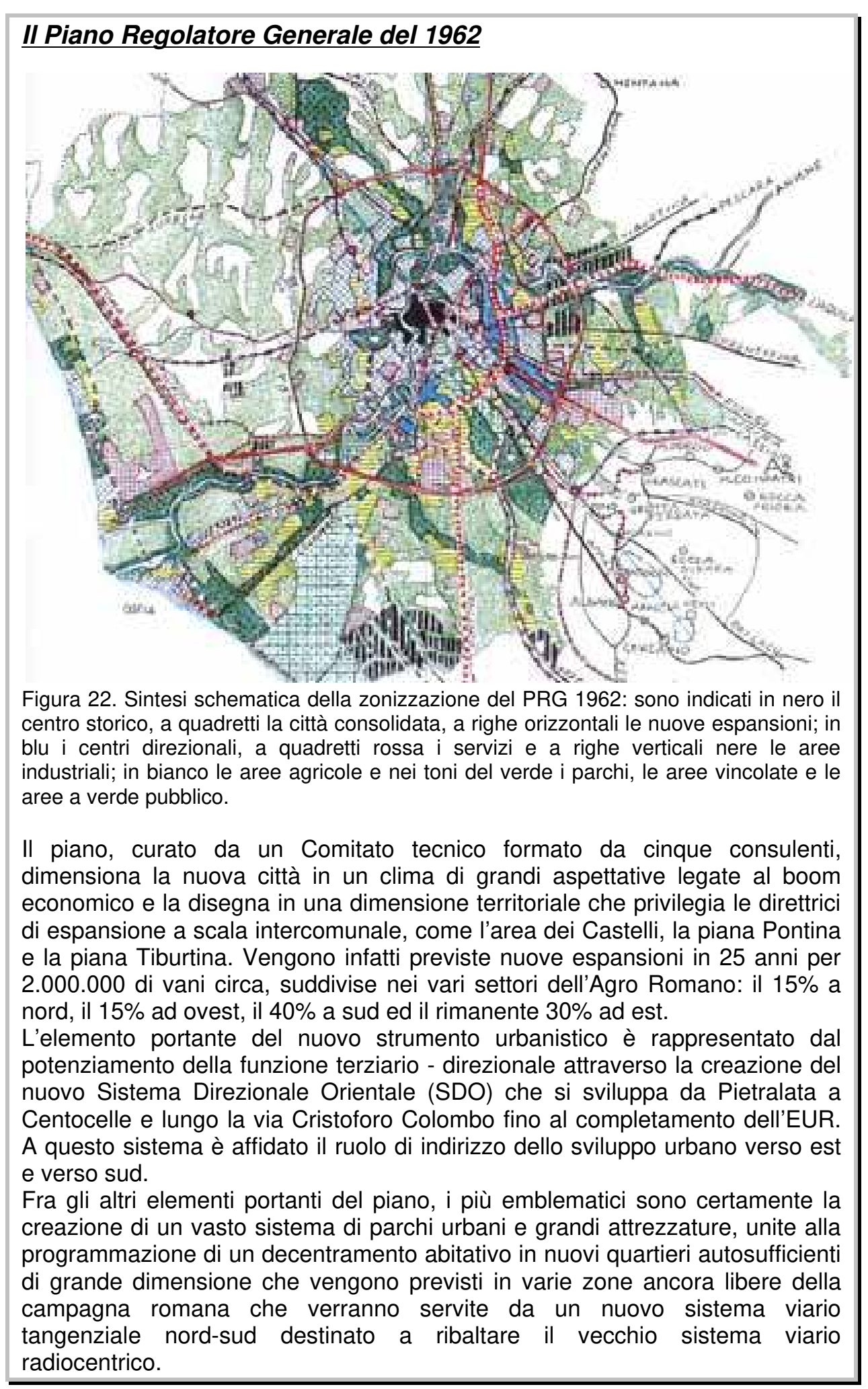


Oggi, con il diffondersi ed il disperdersi delle costruzioni, con l'intensificarsi dei rapporti con i comuni vicini e con l'istituzionalizzazione della dimensione metropolitana, anche gli studiosi di discipline fisico naturalistiche accettano talvolta il termine troppo generico di "area romana" al posto di quello - più immaginifico e più legato ad una tradizione culturale - di "campagna romana".

\section{LE “CAMPAGNE” DELL'AREA ROMANA OGGI}

Dalle letture finora condotte sotto diversi punti di vista e da diverse discipline scientifiche, emergono due temi principali riguardo la nostra area: la sua specificità e la sua capacità di cambiare.

Da una parte si può riscontrare, riconoscere e interpretare, analizzandone le componenti storico culturali, economico-sociali, insediative, la specificità di una dimensione territoriale che non ha solo un valore storico-monumentale, ma anche simbolico, e che rappresenta nello stesso tempo un caso fortemente anomalo di rapporto città - campagna.

Parallelamente,

\footnotetext{
V. CALZOLARI, Le interrelazioni e la varietà, in V. CALZOLARI, «Storia e natura come sistema: un progetto per il territorio libero dell'area romana», Àrgos, Roma 1999, p.101.
} 
quest'area dimostra una sua ricchezza e capacità stratificata nel tempo, una sua capacità di trasformazione, che altre parti del contado italiano non hanno avuto $^{2}$ :

diventa allora essenziale analizzare - partendo dalle permanenze e dalle specificità dei luoghi - come le tracce dei cambiamenti che essa ha vissuto si integrino con il complesso dei nuovi elementi che le diverse trasformazioni hanno introdotto, dando origine ad una serie di realtà paesistiche che solo in parte coincidono con la struttura tradizionale della «campagna romana».

In questo capitolo si descrivono i diversi paesaggi agrari che si sono conformati nell'area romana, indagando in ciascuno di essi le relazioni che si instaurano tra aspetti naturali ed elementi antropici, e le combinazioni che vi sono tra strutture morfologiche e tracce e regole storiche.

La «campagna romana» viene qui articolata in «campagne»: per alcune sue porzioni significative si fa una dettagliata descrizione dei diversi elementi componenti il paesaggio agrario e delle relazioni che compongono la struttura specifica di ciascuna tipologia individuata:

- la "campagna delle tenute», ovvero quelle porzioni di agro romano in cui sono ancora riconoscibili gli elementi caratterizzanti quella particolare forma di conduzione agricola;

- la "campagna della bonifica», localizzata in una parte precisa del territorio romano e ancora facilmente riconoscibile in molti tratti come regola strutturante il paesaggio;

2 G. G. Rizzo nel suo intervento al seminario «Strumenti e metodi per la conoscenza della campagna romana», Dottorato di ricerca in Progettazione Paesistica, Roma 2000. 
- la "campagna della città metropolitana» con la quale si è voluto indicare le realtà "nuove" della campagna romana, originate dallo stretto rapporto tra le componenti ambientali e paesistiche e le componenti più propriamente urbane. 


\section{LA CAMPAGNA DELLE TENUTE}

Il territorio all'interno delle mura Aureliane al momento della breccia di Porta Pia non era del tutto edificato, ma tra la cinta muraria e la città costruita vi era un ampia cintura di ville patrizie abitate dall'aristocrazia romana. Al possesso di ampie zone di territorio urbano da parte di poche famiglie corrispondeva anche una suddivisione in poche ed ampie tenute del territorio del Suburbio e dell'Agro.

La suddivisione storica in Urbe, Suburbio e Agro era funzionale alla vita della città urbanizzata. Fuori le mura la campagna era suddivisa in tenute, la cui proprietà era generalmente degli ordini religiosi, dei capitoli delle diverse chiese e di poche famiglie. (...) Le tenute più prossime alle mura erano per lo più suddivise in piccole parti date ciascuna in enfiteusi perpetua. Questi terreni immediatamente fuori dalle porte della cinta muraria erano destinati ad attività produttive come valche (tintorie) e mole nei pressi dei numerosi fossi che confluivano nel Tevere, cave di sabbia, tufo travertino e pozzolana e infine vigne. $^{3}$

Confrontando le carte del Catasto Gregoriano redatte intorno al 1820 con la Carta dell'Agro Romano del 1913 di Pompeo SPINETTI, si coglie una prima mutazione subìta dalle tenute della campagna romana in quegli anni: nel 1913, infatti, esse sono aumentate di numero ma hanno avuto un forte ridimensionamento dell'estensione. Anche se la proprietà risulta frammentata, l'uso del territorio è però rimasto pressoché immutato.

Le principali ragioni di questo cambiamento sono da attribuirsi alla liquidazione dell'asse dei beni ecclesiastici, che fece sì che gran parte delle tenute di proprietà della Chiesa fosse acquistata dai «mercanti di campagna», dall'aristocrazia e dalla borghesia mercantile rurale romana, da alcuni gruppi della borghesia imprenditoriale del nord Italia.

3 P. Roccasecca, Urbe, Suburbio e Agro: confini in movimento, dal sito digilander.libero.it/aperture/articoli/2.17.html. 
Il cambiamento più consistente che portò al frazionamento ed alla relativa scomparsa delle tenute nella campagna romana fu però dovuto alla crescita - per lo più incontrollata - dell'urbanizzazione nel territorio dell'agro.

A seguito di questo fenomeno (ampiamente descritto nel capitolo precedente) quella sorta di "cerchia" di tenute che circondava il suburbio degli orti e delle ville di Roma ha ormai perso la sua continuità, ma è ancora possibile individuare alcune tenute che - anche se limitatamente - danno origine ad un vero e proprio sistema.

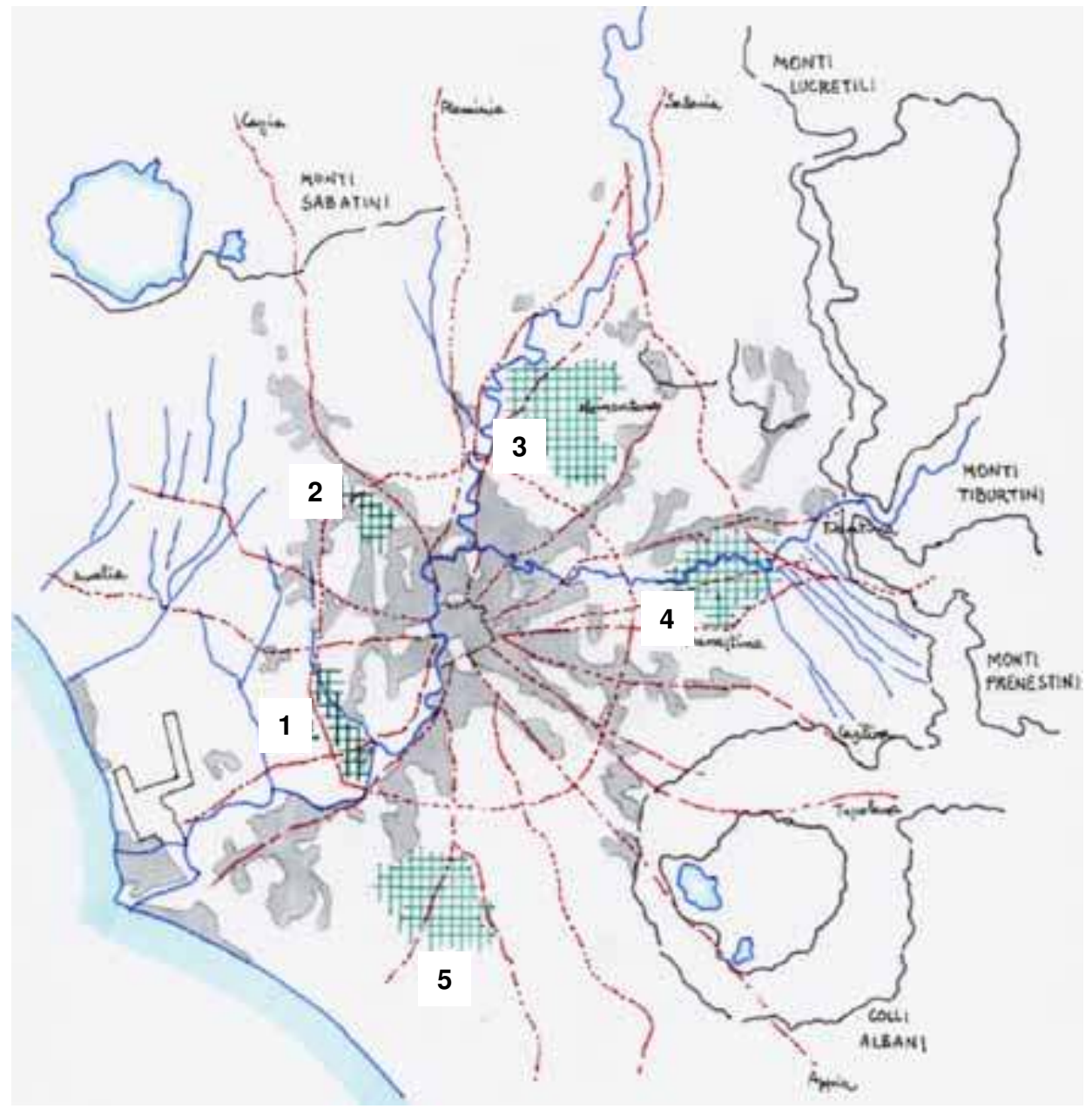

Figura 23. II sistema delle tenute analizzate: 1. Tenute Somaini, dei Massimi, Campo di Merlo, Magliana vecchia e Pisana; 2. Tenuta dell'Insugherata; 3. Tenute della Marcigliana, Tor S. Giovanni, Bufalotta e Casale delle Donne; 4. Tenute di Castel Arcione, Cavaliere e Lunghezza; 5. Tenute di Decima, Monte Migliore, Radicelli e Falcognana. 
Le «campagne delle tenute» che vengono successivamente descritte $^{4}$ non vogliono rappresentare un elenco esaustivo delle tenute ancora esistenti nel territorio dell'area romana, ma costituire esempi del tentativo di rintracciare in quelle porzioni di territorio elementi componenti e relazioni comuni e ricorrenti.

Settore sud ovest: le tenUte Somaini, deI MAssimi, Campo dI Merlo, magliana vecchia e Pisana

Nella Carta dell'Agro Romano del 1913 di Pompeo Spinetti e nell'allegato elenco vengono indicate in questa zona le tenute della Torretta Massimi, di Campo di Merlo, della Magliana e della Pisana, che dai riferimenti storici risultavano in larga parte proprietà dell'Arcispedale del Santo Spirito in Saxia, dei Principi Filippo Lancellotti e Oberto Pallavicini e della Società Agricola Immobiliare Veneta.

L'area che viene di seguito descritta corrisponde oggi in larga parte all'attuale Riserva naturale della tenuta dei Massimi.

\section{La morfologia e l'idrografia dei terreni}

L'area si sviluppa lungo il bacino idrografico del fosso della Magliana, che, con il suo andamento non troppo sinuoso sfocia direttamente nel Tevere all'altezza della nucleo della Magliana Vecchia;

$4 \quad$ Le tenute che vengono analizzate nei loro elementi componenti il paesaggio agrario sono, procedendo in senso orario da ovest ad est: le tenute Somaini, dei Massimi, Campo di Merlo, Magliana vecchia e Pisana; la tenuta dell'Insugherata; le tenute della Marcigliana, Tor S. Giovanni, Bufalotta e Casale delle Donne; le tenute di Castel Arcione, del Cavaliere e di Lunghezza; le tenute di Decima, Monte Migliore, Radicelli e Falcognana. 
a ridosso del fiume, sui due versanti opposti, si trovano le tenute Somaini e dei Massimi.

Nelle immediate vicinanze del Tevere, in una zona completamente pianeggiante, sono invece presenti la tenuta della Magliana Vecchia e quella del Campo di Merlo.

Dal punto di vista morfologico, l'area è caratterizzata dall'alternanza di ripiani tufacei strutturati ad albero rispetto al corso d'acqua principale e di valli particolarmente incise e riccamente vegetate. I fossi che danno origine a queste ultime "vallecole" sfociano nel fosso della Magliana che presenta un fondovalle abbastanza ampio.

I rilievi collinari, di modesta altezza (contenuta fra i 50 e i 60 metri), sono impostati su una serie di terreni di origine fluvio-palustre, costituiti da sabbie, ghiaie e argilla. Solo sulla sommità affiorano, con uno spessore ridotto, i prodotti vulcanici a prevalenza tufacea. Ai rilievi si alternano fondovalle occupati dai sedimenti alluvionali recenti del fosso della Magliana e del Tevere.

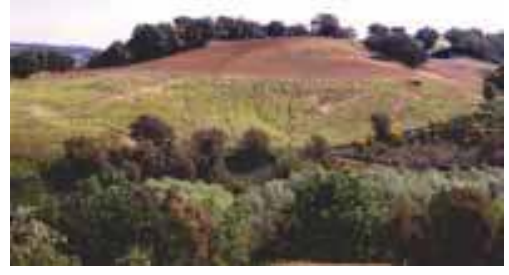

Figura 24. I rilievi collinari.

La porzione di territorio ad ovest è stata interessata da decenni di sfruttamenti dei depositi di ghiaia e sabbia esistenti. Questa attività ha completamente stravolto sia i lineamenti morfologici, sia la distribuzione idrica superficiale e sotterranea della zona.

La morfologia della zona caratterizzata dalle colline arrotondate, dalle modeste quote altimetriche e dall'assenza di cime, unitamente alle scanalature dei fossi ed alle zone pianeggianti, ${ }^{5}$

rappresenta la sequenza strutturale costante dell'intera porzione di territorio. L'ampio fondovalle, solcato per tutta la sua lunghezza dalle linee pressoché parallele del fosso della Magliana e del tracciato stradale omonimo, rappresenta una sorta di spina dorsale di questo

5 G.G. RIzzo, Qualità paesaggistico - ambientali e frange urbane nella periferia ovest di Roma, estratto da «Metodi e verifiche di bilancio ambientale urbano», s.l., 1991, pp.106-107. 
sistema. È dunque oggi ancora possibile riconoscere le forme naturali che modellano il territorio:

- un'estesa superficie pianeggiante di origine alluvionale, generalmente coltivata a seminativo, che si articola lungo il fosso della Magliana e alla confluenza di quest'ultimo con il fiume Tevere;

- una serie di piccole valli con un deciso profilo a "V", originate dal ruscellamento superficiale degli affluenti il fosso della Magliana, che ha profondamente inciso i terreni tufacei;

- una cornice di zone rilevate di origine tufacea, ad un'altezza che non supera i 60 metri, sulle quali si attestano piccole macchie arboree.

\section{I "residui" ambientali e/o storici}

Nel complesso l'area mostra una consistente presenza di complessi forestali ben strutturati, ricchi di numerose specie vegetali e, relativamente al fatto che si è nelle immediate vicinanze dell'agglomerato urbano, estesi su ampie superfici.

Di particolare interesse sono soprattutto le aree boschive (l'esempio forse più pregevole è costituito dal bosco Somaini) che si sviluppano lungo i versanti e sulla sommità dei pendii, dove prevalgono i boschi di sughera e farnetto o quelli formati in prevalenza dal cerro.

Sulle altre parti del territorio prevalgono, invece, le specie di graminacee caratteristiche delle colture abbandonate e dei pascoli aridi; nella zona di confluenza tra il fosso della Magliana e il Tevere, infine, è maggiormente presente la vegetazione altoerbacea e arbustiva delle zone umide.

La permanenza storica più rilevante è rappresentata dal tracciato storico d'epoca romana della via della Magliana, lungo la quale si 
trovano, anche se non indiretta connessione, il complesso dei Fratres Arvales, le catacombe ebraico - cristiane di Generosa e un'area archeologica - sempre di epoca romana - recentemente scoperta in prossimità dell'azienda agricola dell'Infernaccio.

Lungo la stessa strada sono poi presenti due importanti complessi che risalgono al Medioevo: il casale della Torretta dei Massimi che con la pieve annessa rappresentava l'alloggio di guardia all'interno della tenuta dei Massimi, ed il complesso delle scuderie della Magliana, trasformate nel 1503 in villa e residenza di caccia papale ed occupate oggi dalla Congregazione dei cavalieri di Malta.

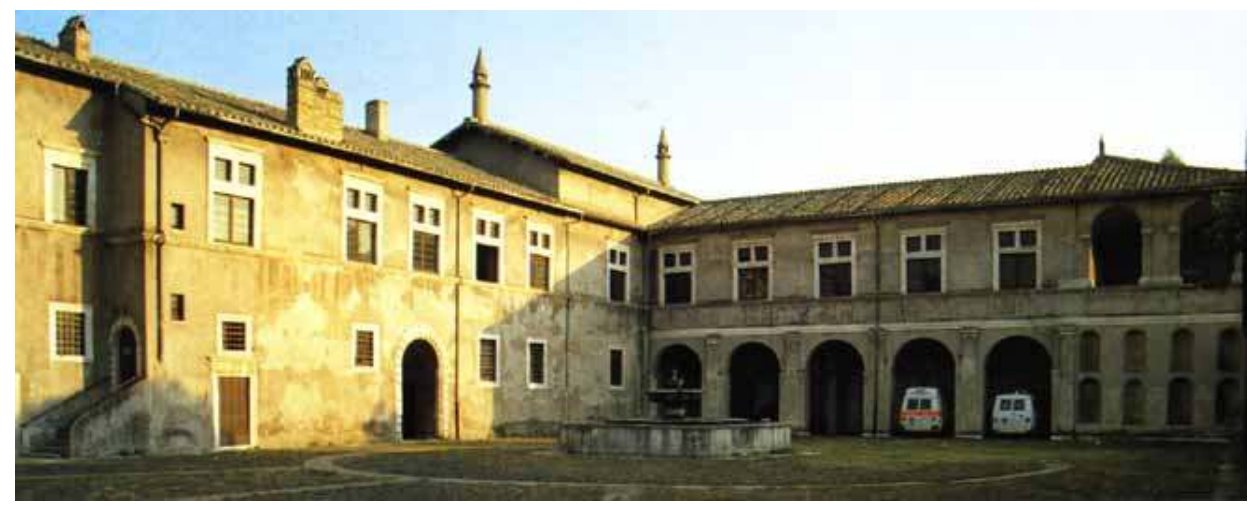

Figura 25. II cortile interno delle Scuderie della Magliana.

Nelle parti più interne sono presenti una serie di casali storici disposti lungo un percorso di crinale che corre parallelamente al fosso della Magliana. Si tratta di un tracciato storico che collega i casali più antichi, documentati già nel Catasto Gregoriano e appartenenti tutti alla tenuta dei Somaini: un casale-villa con torre merlata e altri due casali utilizzati probabilmente come fienile e per le attività legate alle vigne. Tutte queste strutture sono oggi inutilizzate e versano in un forte stato di degrado. 


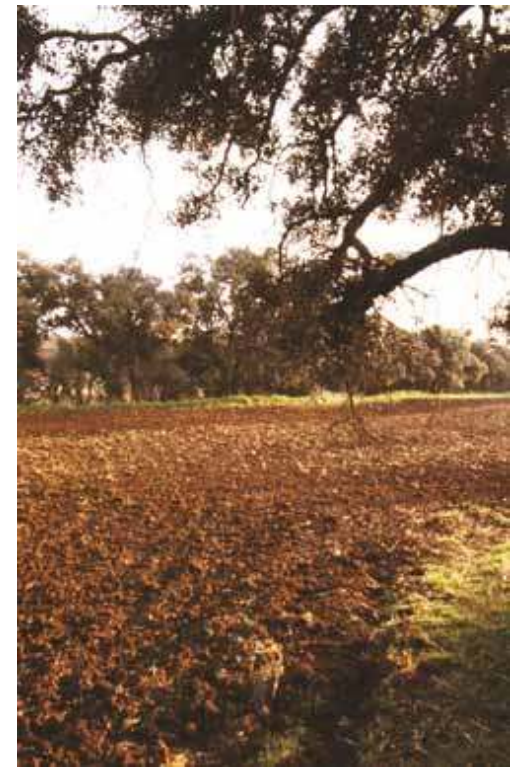

Figura 26. Macchia arborea accanto ad un campo coltivato.

\section{Le sistemazioni agrarie}

II modello di colonizzazione agricola dell'intera zona sembra corrispondere alla sequenza caratteristica della morfologia propria della campagna romana, con una fase iniziale di frequentazione di tipo pastorale a partire dalla sommità dei pianori, alla quale sono seguite un'affermazione della cerealicoltura $e$, in epoche più recenti, una conquista dei fondovalle anche a seguito dell'introduzione dell'aratro profondo.

Di grande rilevanza è, dunque, la presenza di bosco sia su gran parte dei pendii, sia su molte delle sommità dei pianori, alternate ad ampie zone aperte del sistema agricolo moderno dei seminativi nei fondovalle pianeggianti della Magliana, della Maglianella e della piana del Tevere.

La caratteristica principale di questa porzione di territorio non consiste dunque in particolari sistemazioni agricole, quanto piuttosto nell'alternanza dei terreni coltivati con prati e pascoli e con boschi.

L'uso agricolo del suolo che caratterizza il fondovalle presenta ampi appezzamenti di campi seminati a grano, con le colture che strutturano il paesaggio agrario della vallata che ne delineano il margine e avvolgono le aree di pregio naturalistico dei lembi vegetati presenti lungo le valli strette del reticolo idrografico.

La tessitura dei campi, come detto abbastanza ampi, ha un'orditura perpendicolare all'andamento del fosso e dà origine ad una sorta di struttura ad albero rispetto a questo.

Le coltivazioni che vengono effettuate sono per lo più quelle 
della classica rotazione della campagna romana, che vede alternarsi il grano duro, gli erbai autunno - vernini di avena e trifoglio e l'erba medica, che occupa il terreno dai tre ai cinque anni. ${ }^{6}$

La maggior parte delle coltivazioni è in asciutto, anche se in qualche zona è possibile trovare delle piccole superfici coltivate a frutteto e ortaggi per le quali è indispensabile la pratica irrigua.

Le porzioni di terreno poste alle quote relativamente più alte, invece, sono lasciate a prato e pascolo, in quanto ci si trova di fronte a terreni di minore fertilità, spesso di più difficile lavorabilità a causa delle pendenze.

\section{L'insediamento rurale e la tipologia delle aziende}

L'invaso del fondovalle del fosso principale è segnato dal susseguirsi dei complessi dei casali, i quali sono il più delle volte formati da un insieme di edifici differenti, collegati fra loro da una rete di percorsi che attraversa tutta l'area e che struttura anche i principali collegamenti fra le varie tenute.

La viabilità minore interna è limitata al collegamento delle aziende agricole con le grandi arterie di scorrimento, mentre la viabilità interpoderale è abbastanza scarsa e difficilmente percorribile.

La maggior parte dei casali attivi presenti è di epoca moderna: essi sono attestati in prevalenza lungo il fondovalle, quasi ad intervalli regolari, appoggiati sulle prime pendici dei rilievi.

La tipologia insediativa storica ricorrente è quella che vede il casale posizionarsi al fondo di un viale alberato di ingresso, al centro di un ampio spiazzo, protetto da un fitto "schermo verde", intorno al quale non

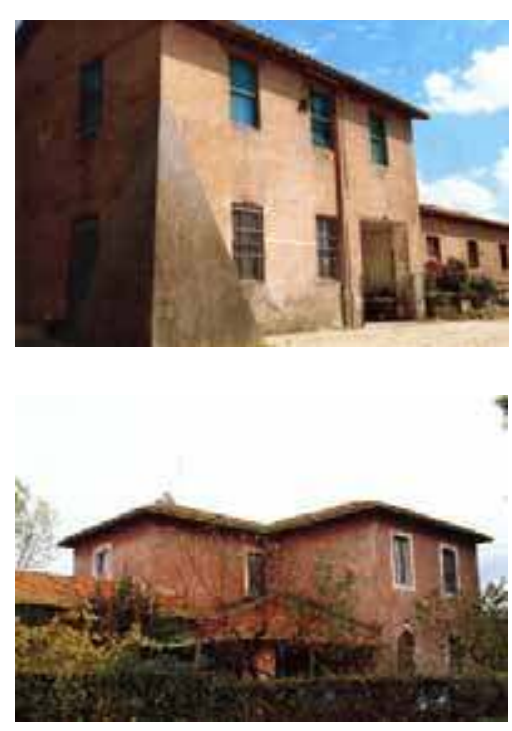

Figure 27-28. Due esempi di casali presenti lungo il fosso della Magliana.

6 G. CAFIERo (a cura di), Paesaggio, natura e agricoltura di fronte alla pressione urbana. Tenuta dei Massimi, in «Urbanistica Quaderni» n.37, mar., INU edizioni, Roma 2003, p.138. 


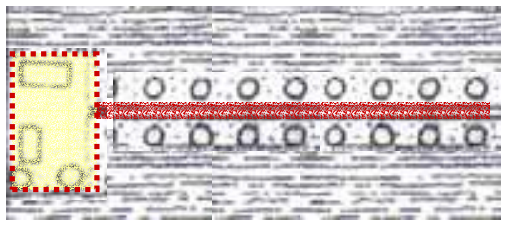

Figura 29. Unità insediativa agricola al fondo del viale alberato d'ingresso. è inusuale ritrovare anche altri edifici di servizio all'attività agricola e pastorizia.

Questi edifici, però, in alcuni casi possono anche essere ubicati in altre parti del territorio della tenuta, al centro di radure o sui pianori meno ricchi di vegetazione.

All'interno delle tenute opera, attualmente, un insieme di aziende di piccole e medie dimensioni che praticano, come detto, un'agricoltura estensiva per lo più in rotazione: al fianco dei vari appezzamenti coltivati sono stati costruiti nuovi fabbricati, non eccessivamente grandi, adibiti a residenza del contadino.

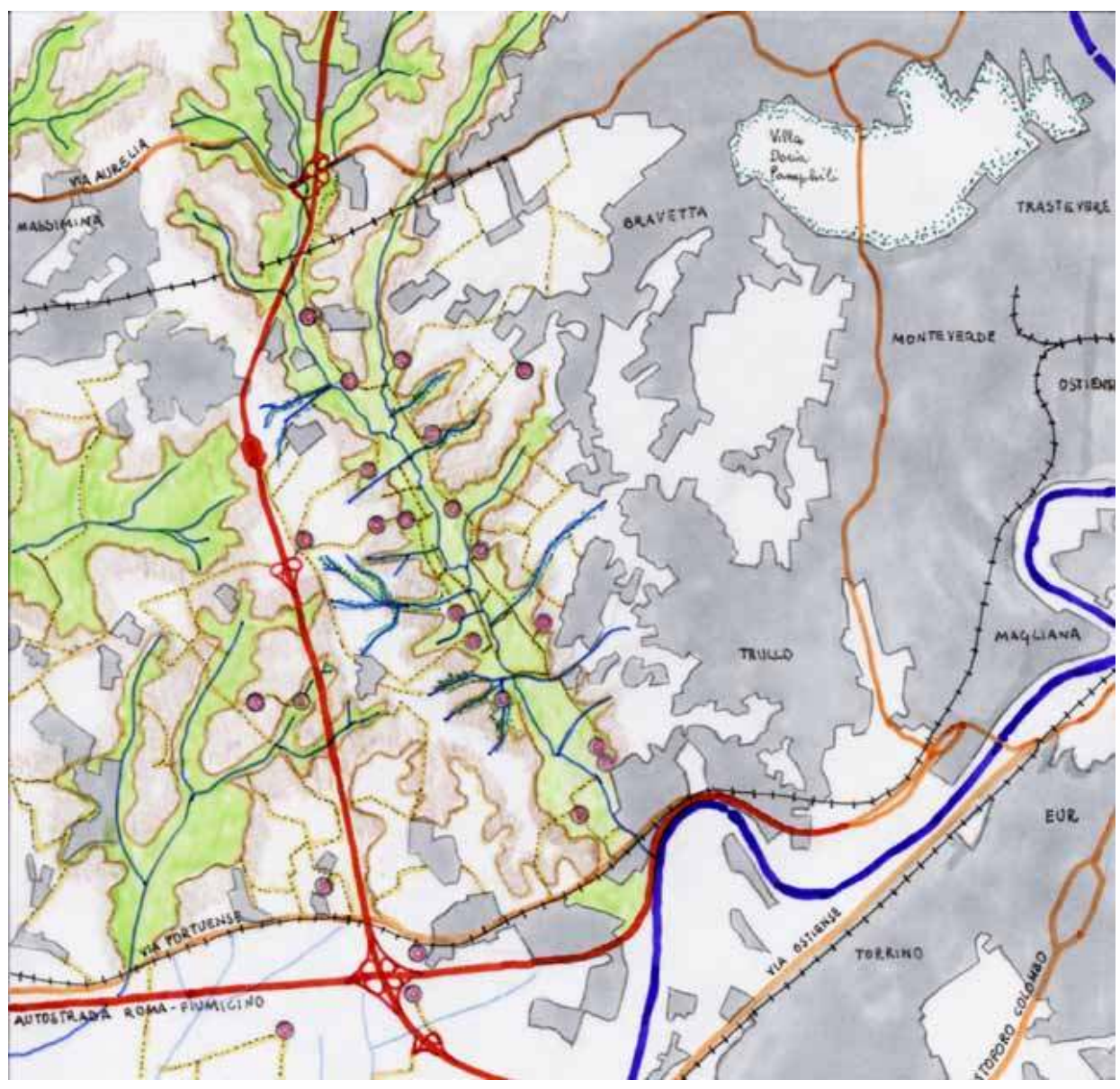

Figura 30. La "campagna" delle tenute del settore sud-ovest: elementi componenti e relazioni. In blu e azzurro è evidenziato il sistema delle acque; in rosso e arancio la viabilità principale; in giallo la viabilità interpoderale e con un pallino rosso le unità insediative agricole dei casali; in verde chiaro e marrone l'alternanza di valli e pianori; in grigio l'urbanizzato. 


\section{SETTORE NORD OVEST: LA TENUTA DELL'INSUGHERATA}

La tenuta era già identificata nell'elenco e nella Carta dell'Agro Romano del 1913di Pompeo Spinetti con un perimetro che grosso modo coincide con l'attuale perimetro della Riserva naturale gestita dall'Ente RomaNatura.

È una porzione tipica della campagna romana che, oggi, è racchiusa tra i quartieri urbani sorti lungo la Cassia ad est, lungo la via Corina d'Ampezzo in direzione sud e lungo i pianori della via Trionfale ad ovest.

\section{La morfologia e l'idrografia dei terreni}

L'area è articolata intorno alla valle del fosso dell'Acqua Traversa e alle valli secondarie dei suoi affluenti: il fosso della Rimessola, il fosso di Monte Arsiccio e il fosso dell'Insugherata, che sin dalla fine del Seicento ha dato nome alla tenuta.

La morfologia del territorio è caratterizzata da una successione di terreni costituiti da sedimenti marini di natura argillosa che rappresentano il lito-tipo più antico affiorante nell'area romana e che emergono nelle aree più depresse e lungo le principali incisioni vallive. Al colmo dei rilievi collinari affiorano, invece, prodotti vulcanici quali i tufi stratificati, provenienti dall'apparato dei monti Sabatini.

La natura prevalentemente impermeabile dei terreni affioranti (le argille marine) ed il loro assetto morfologico collinare fanno sì che l'area sia scarsa di risorse idriche, anche se nella modesta copertura vulcanica sono presenti alcune piccole falde idriche isolate a portata alquanto scarsa.

Nonostante l'ubicazione della tenuta sia all'interno di zone particolarmente insediate, è ancora oggi possibile riconoscere al suo interno le forme naturali del modellamento tipico del paesaggio della 


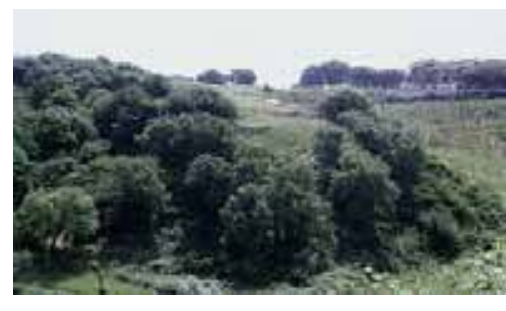

Figura 31. Boschetto di sughera. campagna romana di questa zona. La presenza delle formazioni tufacee al di sopra dei depositi fluviali ha dato origine ad estese superfici pianeggianti, bordate da scarpate particolarmente acclivi. II reticolo fluviale che confluisce nel fosso principale dell'Acqua Traversa ha inciso in maniera profonda $i$ depositi alluvionali e ha formato una serie di vallecole dal caratteristico profilo a "V", alternate ad una serie di superfici terrazzate di origine fluviale.

\section{I "residui" ambientali e/o storici}

La tenuta è, in realtà, caratterizzata dalla presenza più di aree boschive che non di aree coltivate.

La tipologia di bosco più diffusa è quella costituita da concentrazioni di cerro, con farnetto, farnia e sughera a contatto con le aree aperte agricole.

Queste formazioni boschive tipiche si arricchiscono di altre specie vegetali mesofile negli avvallamenti $e$, nelle zone umide lungo le principali linee di impluvio, vengono sostituite da una vegetazione igrofila caratterizzata da salici, pioppi, olmi e robinie.

I principali "residui storici" di epoca romana sono rintracciabili lungo i tracciati della via Cassia e della via Trionfale e sono rappresentati soprattutto dai resti di ville patrizie (come quella attribuita a Lucio Vero e rinvenuta in corrispondenza dell'attuale Villa Manzoni) e di tombe (tomba di M. Vibio Mariano detta tomba di Nerone). Lungo la via Trionfale è inoltre presente il passaggio, in un condotto sotterraneo, dell'Acquedotto Traiano - Paolo.

L'area era sicuramente già coltivata in epoca medievale, tanto che nella parte più centrale della tenuta vi sono i resti di un'antica torre e 
sulla via Trionfale è ancora ben visibile il Casale dell'Insugherata che risale allo stesso periodo storico.

Ricordiamo che nell'XI secolo la via Trionfale costituiva un tratto dell'importantissima via Francigena, percorsa da pellegrini provenienti da tutta Europa.

\section{Le sistemazioni agrarie}

Il paesaggio agrario tipico di questa porzione di campagna romana è quello strutturato dall'alternanza fra boschi di spalletta, fondovalle e pianori coltivati a seminativo. La "regola" sembra essere quella della sequenza alto-basso: la regione a dominanza agricola corrisponde alle quote più alte dei pianori, quella naturalistica alla quote più basse dei fondovalle e dei versanti boscati.

Le coltivazioni prevalenti sono costituite dalle colture tipiche della rotazione (grano duro, erbai di avena e trifoglio, erba medica) che si collocano dunque più frequentemente sui pianori, caratterizzati da terreni con differenti gradi di fertilità, e talvolta nei fondovalle, dove la vicinanza dei fossi permette un'irrigazione continua e dove sono particolarmente interessanti - laddove sono ancora ben mantenute - le sistemazioni idraulico-agrarie delle affossature e dei drenaggi.

A questo tipo di colture prevalenti si associano, di tanto in tanto, delle zone ad uliveto $e$, in una zona posta all'estremità della valle dell'Insugherata, un'area con piccoli orti coltivati ad uso familiare da

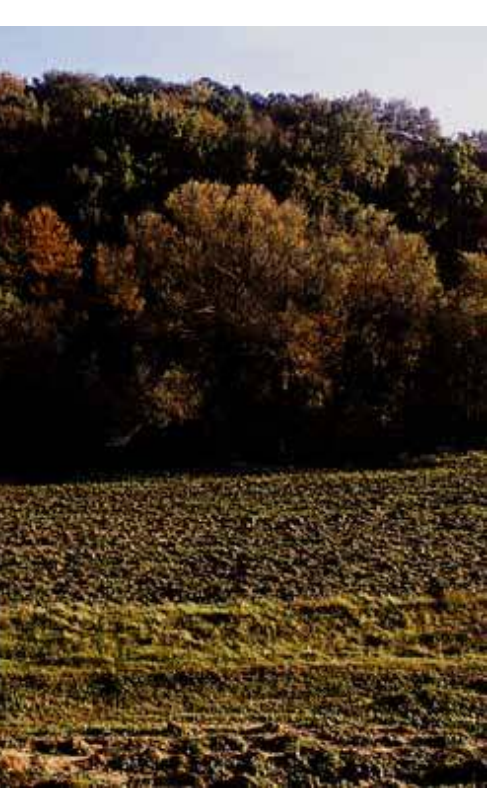

Figura 32. Le coltivazioni agricole dei fondovalle e il bosco di spalletta costituiscono i due elementi ricorrenti componenti il paesaggio agrario di questa persone che abitano nei quartieri vicini. 

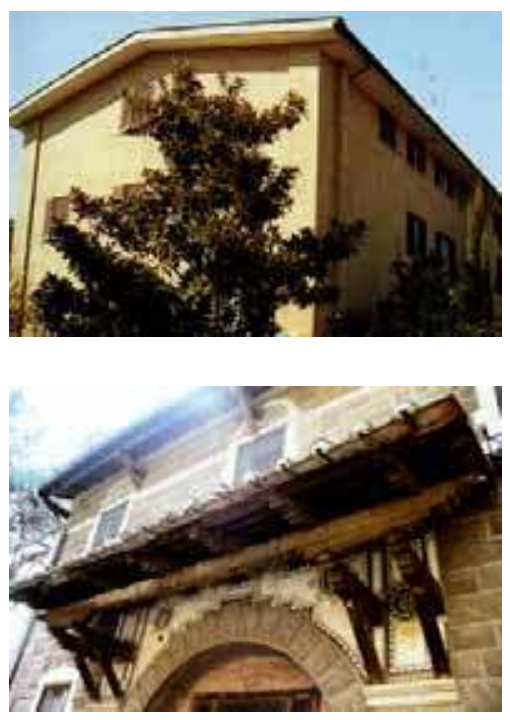

Figure 33-34. Due esempi dei fabbricati rurali presenti nell'area: il primo è il casale della Giustiniana, il secondo è il casale Maiolica.

\section{L'insediamento rurale e la tipologia delle aziende}

Nella parte centrale del territorio della tenuta si conferma, come detto, la vocazione agricola e silvopastorale che sin dai tempi antichi caratterizzava l'area, anche se le strutture insediative agricole sono sempre state ritenute di scarsa importanza, forse a causa dell'insalubrità dei luoghi dovuta alla presenza del fosso dell'Acqua Traversa e dei suoi affluenti. All'inizio del 1800 vengono infatti indicati dal Catasto Gregoriano solo il casale della Giustiniana e l'osteria ad esso annessa, al bivio tra la via Cassia e la via Trionfale.

Con le bonifiche dell'epoca fascista e la fondazione della borgata rurale di Ottavia si consolida il sistema agricolo insediativo attuale, formato dai casali sorti sui pianori sommitali e posti a presidio del paesaggio agrario e boschivo della zona.

Il patrimonio dei fabbricati rurali è però molto scarso rispetto alla superficie coltivata, tanto che nell'area sono oggi compresi solo quattro insediamenti agricoli:

- il casale Maiolica, al centro della tenuta, composto dall'edificio adibito alla residenza e da un fienile con alcuni limitati annessi agricoli;

- il centro agricolo Bufolareccia, costituito da un grande casale e da un capannone adibito a scopi prevalentemente agricoli;

- il centro agricolo Vignaccia, con un casale affiancato ad una ex stalla, una serie di piccoli casali più recenti e alcuni annessi agricoli;

- il casale Gambino, formato da un gruppo di fabbricati che sono stati recentemente ristrutturati a fini esclusivamente residenziali.

La caratteristica peculiare della conduzione agricola nella tenuta dell'Insugherata è costituita dal fatto che si è mantenuto al suo interno, sostanzialmente inalterato, un insieme particolare e unico nel suo 
genere di medio-grandi aziende cerealicole e zootecniche estensive, accorpate e attive, in un'area densamente urbanizzata.

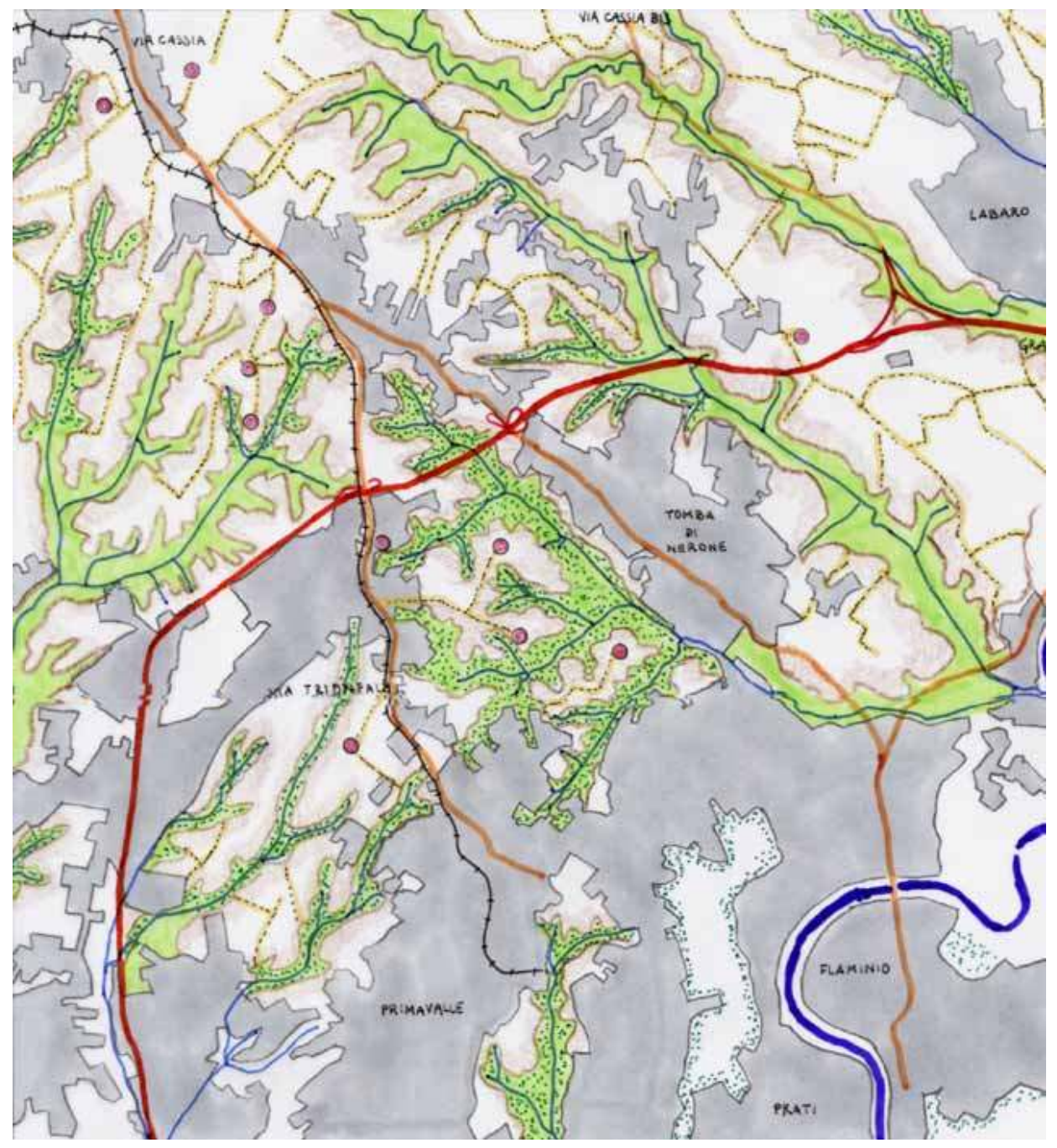

Figura 35. La "campagna" delle tenute del settore nord-ovest: elementi componenti e relazioni. In blu e azzurro è evidenziato il sistema delle acque; in rosso e arancio la viabilità principale; in giallo la viabilità interpoderale e con un pallino rosso le unità insediative agricole dei casali; in verde chiaro e marrone l'alternanza di valli e pianori; in grigio l'urbanizzato. 
Settore nord: Le tenute della Marcigliana, TOR S. GiovanNI, Bufalotta e CASALE DELLE DONNE

Le due tenute della Marcigliana e di Tor $\mathrm{S}$. Giovanni erano già indicate con questo nome da Pompeo Spinetti nel 1913 nella Carta dell'Agro Romano: la prima era di proprietà del Duca Mario Grazioli, la seconda dell'Arcispedale del S.S. Salvatore al Sancta Sanctorum.

Oggi parte di queste tenute rientra nel perimetro della Riserva naturale della Marcigliana, gestita dal 1997 dall'Ente RomaNatura.

Si tratta di una zona esterna al Grande Raccordo Anulare, che conserva in gran parte un forte carattere rurale. Da questo punto di vista, però, è un'area di relativo sottosviluppo, causato soprattutto dal fatto che l'attesa nel corso degli anni di una rapida rivalutazione immobiliare legata all'espansione urbana ha certamente costituito un deterrente a qualsiasi intenzione e tentativo di sviluppo di progetti e di investimenti nel settore agro-ambientale.

\section{La morfologia e l'idrografia dei terreni}

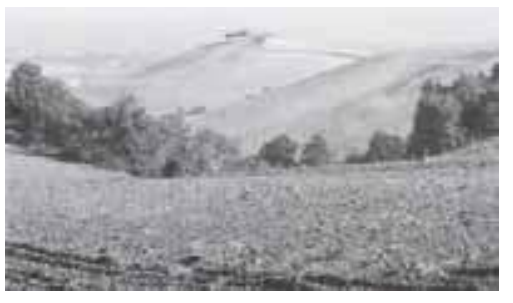

Figura 36. L'alternanza dei rilievi che caratterizza questa zona.
La parte centro-meridionale di questa porzione di territorio ospita i prodotti vulcanici provenienti dagli apparati eruttivi dei monti Sabatini e dei Colli Albani e costituiti da tufi litoidi e pozzolane di vario colore. Questi materiali hanno, in epoche alquanto remote, colmato e livellato una morfologia preesistente molto articolata, raggiungendo talvolta spessori di alcune decine di metri. La parte settentrionale, invece, è topograficamente più rilevata e poggia su uno strato di argille e marne di origine marina.

In particolare, i suoli dei pianori sommitali sono prevalentemente vulcanici e profondi, mentre i versanti sono caratterizzati da una profondità limitata degli stessi prodotti vulcanici. 
I versanti ubicati nel limite più a nord dell'area, invece, presentano dei suoli moderatamente o molto calcarei a tessitura fina, con un substrato di argilla, limo e, in alcune piccole parti, travertino.

La successione delle formazioni vulcaniche con quelle terrigene ha dato origine ad una morfologia del territorio variabile ed articolata nelle sue parti, con zone rilevate a volte pianeggianti sulla sommità, i plateau tufacei, separate da valli particolarmente incise a causa dell'azione erosiva delle acque di ruscellamento, con la conseguente formazione di terrazzi fluviali ben evidenti.

\section{I "residui" ambientali e/o storici}

La zona, pur essendo interessata in prevalenza dalle coltivazioni agricole, presenta alcuni "residui" ambientali di notevole importanza: sono per lo più boschi che si sono mantenuti quasi esclusivamente in corrispondenza dei versanti del sistema collinare formati da cerro combinato con ornello, acero, roverella e carpino sulle pendici, con farnia ai piedi dei versanti.

Lungo i fossi principali, poi, è possibile trovare alcuni lembi della vegetazione arborea igrofila con salici e pioppi.

Questi territori custodiscono un patrimonio archeologico che è sicuramente meno grandioso delle vestigia della Roma imperiale, ma non è meno importante per quanto riguarda la ricostruzione della storia del territorio. Ogni epoca storica ha, infatti, lasciato la sua impronta ed il suo sistema di relazioni: la progressiva sovrapposizione di tutti questi segni ha dato vita ad una struttura particolare dell'intero territorio.

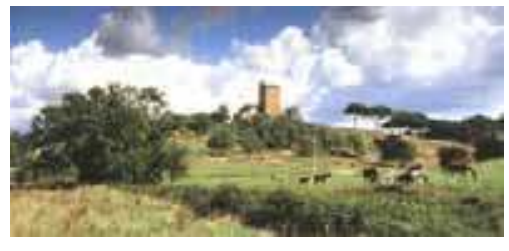

Figura 37. La torre di S. Giovanni.

Sui pianori che oggi sono attraversati dalla via della Marcigliana sorgevano, ad esempio, l'antica città pre-romana di Crustumerium, che 
durante il periodo repubblicano raggiunse quasi i 10.000 abitanti, e poco distante l'antico insediamento di Fidene, sorto in epoca arcaica.

Di questo passato, dunque, l'area conserva ancora un ricco patrimonio di reperti, quali tracciati viari, necropoli, sepolture, ruderi di ville romane, ecc...

Sono presenti anche resti di fortificazioni medievali: la torre di Tor S. Giovanni, che dà il nome alla tenuta; la torre della Marcigliana, che fu prima inglobata in un casale e successivamente (XVI - XVII secolo) nell'omonimo castello; altre torri e torrette con funzioni meramente difensive, come la torre di Redicicoli.

\section{Le sistemazioni agrarie}

Le pratiche agricole rappresentano, senza ombra di dubbio, l'uso più visibile e caratteristico dell'intero territorio: le coltivazioni orticole e le colture legnose costeggiano solitamente i percorsi interni, in prossimità delle tipologie dell'insediamento rurale (casali e aziende agricole).

La parte sud è caratterizzata dalla presenza di appezzamenti di grande estensione, a memoria dell'antico sistema del latifondo proprio di tutta questa zona. II paesaggio agrario è qui connotato dalle vaste distesee assolate dei pianori dove regnano le monocolture estensive: sono i grandi latifondi delle tenute agricole storiche (Capobianco, Cesarina, Oleole, Bocconcino), con gli appezzamenti coltivati ripartiti in forme geometriche da strade alberate, filari e siepi.

Nella porzione a nord del territorio, invece, i fondovalle sono solitamente coltivati a seminativo o adibiti a prati e pascolo permanente, anche se, in sporadici casi, gli appezzamenti a seminativo estensivo si "arrampicano" anche sui versanti fino ad una certa quota, dove le colture sono poi sostituite dai boschi di cerro o di roverella. 
II paesaggio agrario di questa zona potrebbe, dunque, essere distinto ne:

- la zona a sud, caratterizzata da colline molto dolci, con suoli vulcanici particolarmente fertili, dove, oltre gli ampi appezzamenti coltivati a seminativo, sono presenti vaste superfici di ulivi e vigneti;

- la zona a nord, oltre il fosso di Settebagni, dove i rilievi sono più pronunciati, le valli sono più strette e irregolari e fiancheggiano fossi dal corso d'acqua particolarmente tortuoso, le spallette boscate costituiscono la separazione fra i diversi pianori con appezzamenti di media estensione coltivati a seminativo;

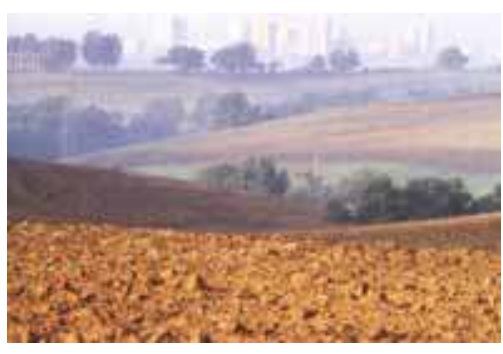

Figura 38. I pianori coltivati a seminativo, solcati dalla vegetazione di vallecole alluvionali.

- la zona all'estremo limite nord dell'area, che presenta una morfologia completamente difforme dal resto del territorio con pendenze notevoli, sulle quali il paesaggio è caratterizzato da filari di pini e macchie di uliveti;

- la zona della vale fluviale del Tevere, caratterizzata da terreni di origine alluvionale, particolarmente fertili, sui quali vi sono appezzamenti di coltivazioni irrigue, orditi radialmente rispetto al corso del fiume.

A partire dagli anni '70 le superfici foraggere sono drasticamente diminuite, modificando il tradizionale paesaggio agrario della zona, e sono state sostituite dalle coltivazioni di girasole, che arricchiscono di variazioni cromatiche la campagna. La pastorizia è, infatti, divenuta via via meno redditizia, tanto che si è assistito ad una graduale scomparsa della zootecnia, che ha subìto un generale processo di abbandono e si è concentrata in poche grandi aziende specializzate.

\section{L'insediamento rurale e la tipologia delle aziende}

II territorio è punteggiato da unità insediative agricole di varie dimensioni e tipi, che vanno dal singolo casale accompagnato da 


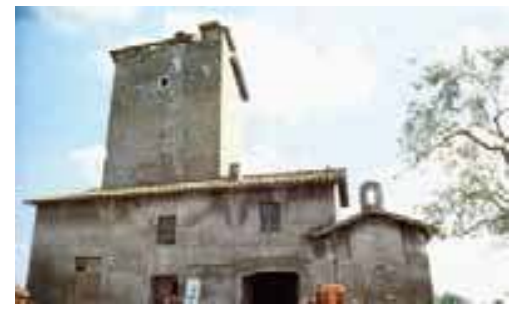

Figura 39. Casale Tor S. Giovanni. strutture di servizio, alla grande azienda agricola completa di stalle e recinti per gli allevamenti.

Quasi tutte le unità insediative agricole presenti, comunque, sono nate specificatamente per la gestione dei fondi e sono quindi dislocate in maniera strategica sul territorio: lungo le linee di crinale, sui rilievi isolati, alla confluenza di corsi d'acqua, ovvero in tutti quei posti dove è possibile svolgere il ruolo di presidio territoriale e controllo vigile delle coltivazioni.

La rete dei casali odierna ricalca infatti quella antica che si era andata via via posizionando lungo i sentieri di crinale, preferendo, per il presidio del territorio, la linea di cresta piuttosto che i luoghi della vigilanza puntuale alla confluenza delle valli. Dalle propaggini più alte, infatti, le estesissime coltivazioni del latifondo sono tenute costantemente e facilmente sotto controllo; i terreni tufacei sono inoltre particolarmente fertili ed adatti alle coltivazioni.

Una tipologia di unità insediativa ricorrente in questa zona è quella dove l'affaccio del crinale è occupato da un casale al servizio del fondo agricolo, eventualmente affiancato da colture arboree e caratterizzato dalla presenza di un viale di accesso alberato.

Una seconda tipologia è quella propria del sistema dei rilievi spartiacque tra le valli del fosso della Regina e di Tor S. Giovanni.

I casali, formati da più corpi edificati ed ubicati sulle propaggini più alte di questo sistema, insistono sulla viabilità di collegamento tra i punti sommitali e sono solitamente contornati o affiancati da boschetti.

Un'ulteriore tipologia caratterizza, a sud, il territorio delle tenute agricole storiche, dove i complessi edilizi dominano i fondi da posizioni centrali e sono attorniati da orti e colture arboree.

Un paesaggio singolare, infine, è quello della tenuta della Bufalotta, dove una torre, i resti di una chiesa medievale, i ruderi di una villa e di alcuni basolati di epoca romana punteggiano le coltivazioni, 
caratterizzate anche da una scarsa presenza di strutture insediative agricole.

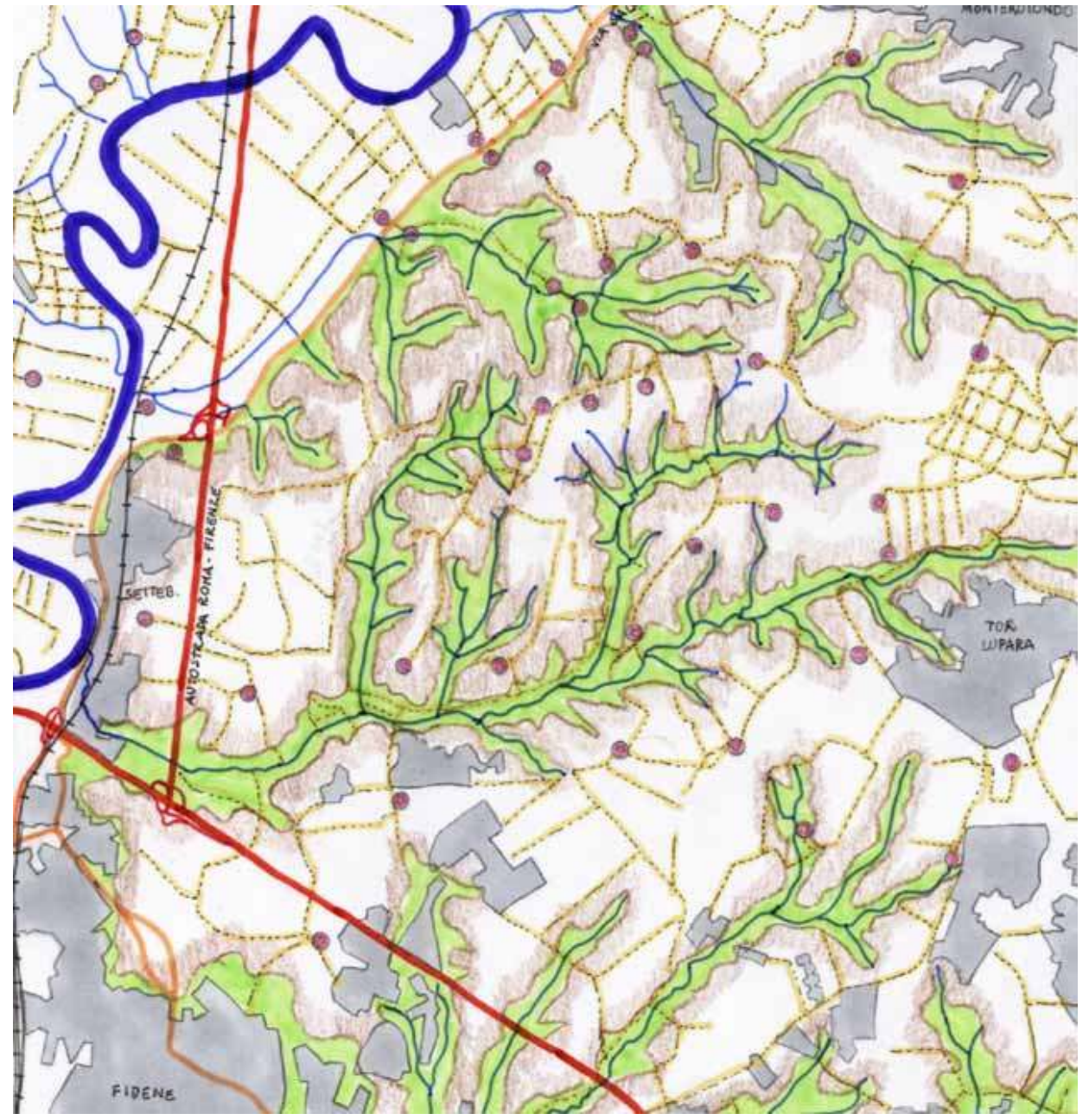

Figura 41. La "campagna" delle tenute del settore nord: elementi componenti e relazioni. In blu è evidenziato il sistema delle acque; in rosso e arancio la viabilità principale; in giallo la viabilità interpoderale e con un pallino rosso le unità insediative agricole dei casali; in verde chiaro e marrone l'alternanza di valli e pianori; in grigio l'urbanizzato. 
Settore est: le tenute di Castel arcione, del Cavaliere e d LUNGHEZZA

Questo settore, da quanto risulta dalla Carta dell'Agro Romano di Pompeo SPINETTI, ancora nel 1913 era particolarmente ricco di tenute, fra le quali probabilmente le più importanti ed estese erano proprio quelle di Castel Arcione, di proprietà della famiglia degli Aldobrandini, del Cavaliere, di proprietà dell'Ospedale Fate Bene Fratelli, e di Lunghezza e Lunghezzina, del Duca Mario Grazioli.

Oggi una porzione del territorio di queste tenute, entro il territorio del Comune di Roma, rientra nel perimetro della Riserva naturale della Valle dell'Aniene.

La zona si presenta come una vera e propria fascia di transizione tra la campagna romana, il sistema vulcanico dei Colli Albani e i rilievi montuosi pre-appenninici rappresentati dai Monti Lucretili.

\section{La morfologia e l'idrografia dei terreni}

L'area costituisce la parte terminale di un importante sistema idrografico, di formazione relativamente recente, centrato sul fiume Aniene e sui numerosi fossi che in esso confluiscono fra Tivoli e Roma.

L'Aniene, dal punto di vista geo-morfologico, segna grosso modo il limite fino al quale ha esercitato la sua azione il complesso vulcanico dei Colli Albani, le cui eruzioni hanno depositato sulla zona che si estende a sud del fiume uno spesso strato di materiali piroclastici, che si sono poi trasformati in banchi di tufo e pozzolana. Queste formazioni vulcaniche recenti hanno così sepolto in profondità gli strati di origine marina più antichi, i quali, invece, caratterizzano le ondulazioni collinari del territorio a nord del fiume, salvo quelle porzioni dove sono stati ancora coperti dai 
depositi alluvionali più recenti prodotti dal fiume e dai sui affluenti durante i fenomeni di piena.

II fiume rappresenta, dunque, l'elemento idro-geo-morfologico strutturante l'intera zona, dividendola in due parti ben distinte tra loro:

- il territorio a nord del fiume - dove ricadono la tenuta di Castel Arcione e la tenuta del Cavaliere - formato da un substrato di sedimenti alluvionali e marini recenti quali travertini, sabbie, ghiaie, limi, ecc...

- il territorio a sud del fiume - dove ritroviamo la tenuta di Lunghezza e Lunghezzina - caratterizzato dalla presenza di numerose forre tufacee originate dall'incisione dei corsi d'acqua sui tufi teneri prodotti dall'attività del Vulcano Laziale.

Una risorsa molto importante, soprattutto sotto il profilo economico, sfruttata su vasta scala sin dai tempi degli antichi Romani, è costituita dai materiali di escavazione (travertino, tufo, sabbie, argille, pozzolane, calcare, ecc...) abbondantemente diffusi su tutta l'area. Di particolare importanza, ad esempio, sono i potenti banchi di travertino, spessi centinaia di metri ed intercalati con strati argillosi, della zona che sta a cavallo della via Tiburtina immediatamente a nord del fiume Aniene, soprattutto nella zona di Bagni di Tivoli, Villanova e Villalba.

Le manifestazioni tettoniche, caratterizzate dalla formazione di faglie e di fratture, dell'area carsica di monte Gennaro, hanno inoltre favorito la risalita dei flussi caldi. Questo bacino è stato, infatti, sfruttato sin dall'antichità per cure termali che ne costituiscono un'importantissima attrazione e risorsa economica. 


\section{I "residui" ambientali e/o storici}

Pur essendo una zona che presenta una copertura vegetazionale quasi essenzialmente agricola, il contesto è ricco di nuclei residuali di vegetazione ripariale lungo i corsi d'acqua principali e secondari.

La vegetazione di tipo legnoso è prevalentemente accantonata a ridosso di una ristretta fascia lungo le scarpate d'alveo, dove rappresenta resti più o meno conservati di comunità di pioppi e salici arborei e cespugliosi.

Particolarmente interessanti sono, poi, gli esempi di piccoli e parziali

popolamenti a olmo e robinia che dimostrano una certa tendenza della vegetazione arborea a rioccupare l'area del sistema agricolo. ${ }^{7}$

Un elemento di rilievo dal punto di vista prettamente botanico è rappresentato da un biotopo localizzato, immediatamente a nord di Ponte Mammolo, su una rupe tufacea e dovuto, probabilmente, a precedenti processi erosivi determinati da un'ansa del fiume. II sito ha un'estensione limitata ed è inglobato all'interno di un tessuto insediativo molto fitto.

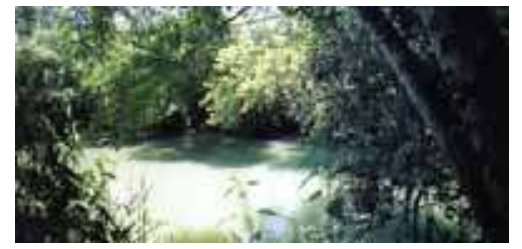

Figura 42. Uno scorcio del fiume Aniene.
La valle del fiume Aniene ha sempre rappresentato la più comoda e naturale via d'accesso da Roma verso l'Abruzzo; il fiume acquistò grande importanza soprattutto in età romana, soprattutto perché da esso proveniva quasi per intero il rifornimento idrico della città: il complesso archeologico più importante della valle dell'Aniene è infatti quello degli acquedotti. I quattro acquedotti principali dell'antica Roma traevano le loro origini proprio dalla valle dell'Aniene: due di essi, l'Anio Vetus e l'Anio Novus, erano alimentati dalle acque stesse del fiume, mentre gli altri due, l'Aqua Marcia e l'Aqua Claudia, nascevano dall'allacciamento

7 G. CAFIERo (a cura di), Ambienti fluviali e comunità urbane. La Riserva naturale della Valle dell'Aniene, in «Urbanistica Quaderni» n.37, mar., INU edizioni, Roma 2003, p.203. 
di alcune sorgenti tra Arsoli ed Agosta. Questi acquedotti sviluppavano un percorso di circa $70 \mathrm{~km}$ che si svolgeva in parte sottoterra, in parte al livello del terreno e in parte in elevazione; una volta giunti a Roma, ogni ramo aveva una sua diversa destinazione.

Resa dunque celebre per le sue acque, tutta la valle si popolò, soprattutto in età imperiale, di magnifiche ville (Augusto, Mecenate, Adriano) e Tivoli stessa divenne soggiorno estivo preferito dai ricchi patrizi fin dagli ultimi tempi della Repubblica.

I numerosi resti di ville, necropoli, tombe, aree archeologiche testimoniano proprio come $\mathrm{e}$ in che misura questo fosse un territorio intensamente occupato ed utilizzato: i "residui" più emblematici sono

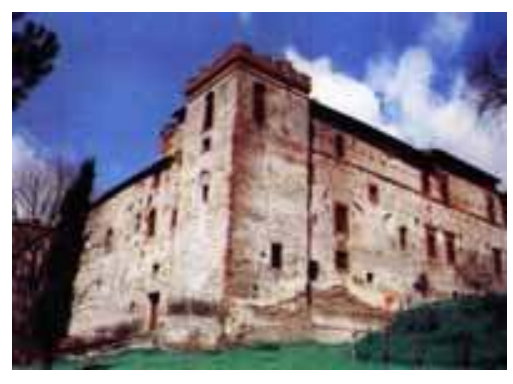

Figura 43. II castello di Lunghezza. rappresentati, oltre che dai numerosi resti di acquedotti, dal castello di Lunghezza e dal ponte Lucano.

\section{Le sistemazioni agrarie}

La differenziazione dei sistemi geografici compresenti nell'area ha prodotto, nel corso del tempo, una articolata diversità di usi agricoli.

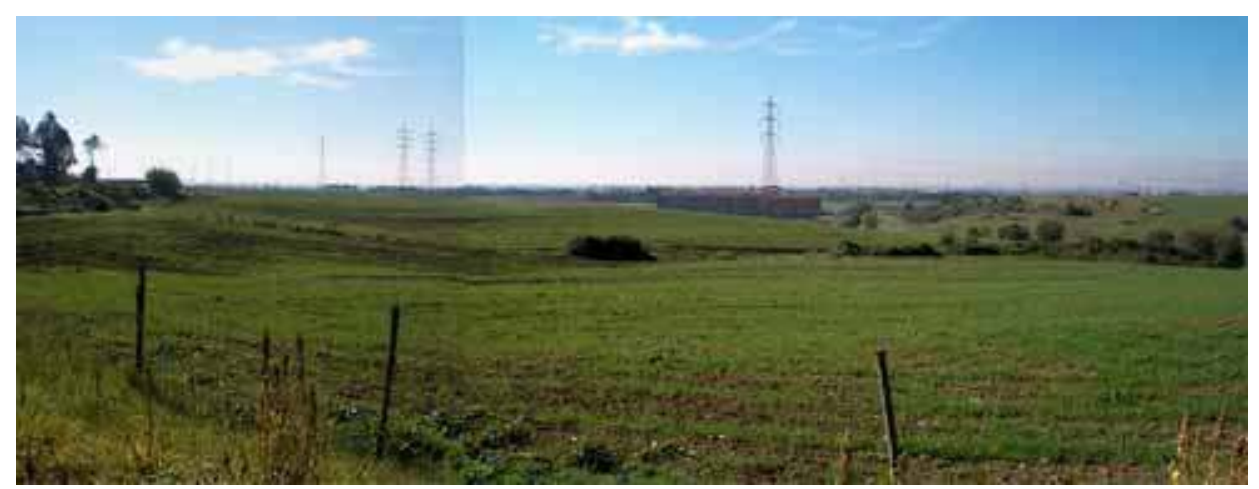

Figura 44. Le leggere ondulazioni caratteristiche della zona a nord dell'Aniene.

Le zone montane, ai margini, sono sempre state destinate prevalentemente al pascolo delle greggi che praticavano la transumanza dalle zone appenniniche; le zone collinari o di media altezza sono state 


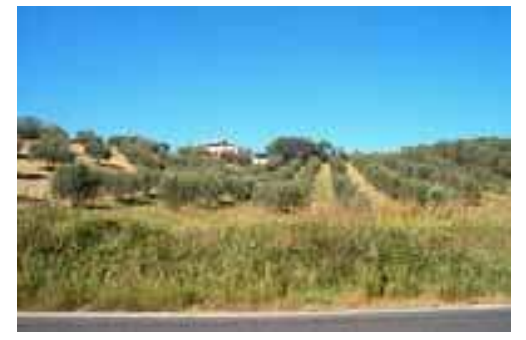

Figura 45. Gli uliveti della parte a sud dell'Aniene. coltivate solitamente a frutteti ed oliveti e presentano una serie di insediamenti arroccati; le zone della valle e della bassa collina, infine, sono coltivate in maniera estensiva.

In particolare, la zona a nord del fiume è caratterizzata da un'agricoltura di tipo estensivo, con gli appezzamenti di medio - grandi dimensioni che seguono l'andamento orografico dolcemente ondulato.

La parte sud, invece, presenta usi agricoli specializzati (uliveti innanzitutto) che si posizionano sulle forre strutturate a pettine rispetto all'andamento del corso del fiume.

Nella parte più vicina alle sponde del fiume, infine, sono presenti ampie proprietà con coltivazioni miste: in particolare quelle orticole che possono attingere l'acqua per l'irrigazione direttamente dal fiume.

\section{L'insediamento rurale e la tipologia delle aziende e della loro conduzione}

Il paesaggio dell'area a sud del fiume Aniene è caratterizzato sin dal Medioevo dalla presenza di una agricoltura di tipo estensivo organizzata intorno ad un vero e proprio sistema di casali. Tra il XII e il XIII secolo, con lo sviluppo del feudalesimo, si ebbe un forte sviluppo di questa costruzione che assunsero una struttura di carattere difensivo. Molti di questi interventi, nonostante le trasformazioni subíte nei periodi successivi - soprattutto recenti - sono ancora leggibili.

La tipologia del casale conserva, in buona sostanza fino ai primi anni del '900, caratteristiche legate alla tradizione edilizia medievale, mentre le costruzioni rurali costruite a seguito delle leggi di bonifica tendono ad assumere caratteri costruttivi più simili alle tipologie urbane, realizzando manufatti che contemperano le esigenze dettate dalla conduzione agricole con le norme igieniche. 
Oggi il sistema dei casali è rimasto pressoché identico a quello del passato, tanto che ancora sono riconoscibili:

- un sistema posto a nord del corso dell'Aniene, dove i casali si pongono al centro della proprietà delle tenute e sono caratterizzati da un lungo viale di accesso alberato, collegato direttamente alla viabilità principale;

- un sistema posto nella valle del fiume e sui pianori a sud di esso, dove i casali, avendo rivestito soprattutto una funzione difensiva, mantengono ancora alcuni dei caratteri di fortificazione e sono generalmente posti sulle propaggini più alte del territorio.

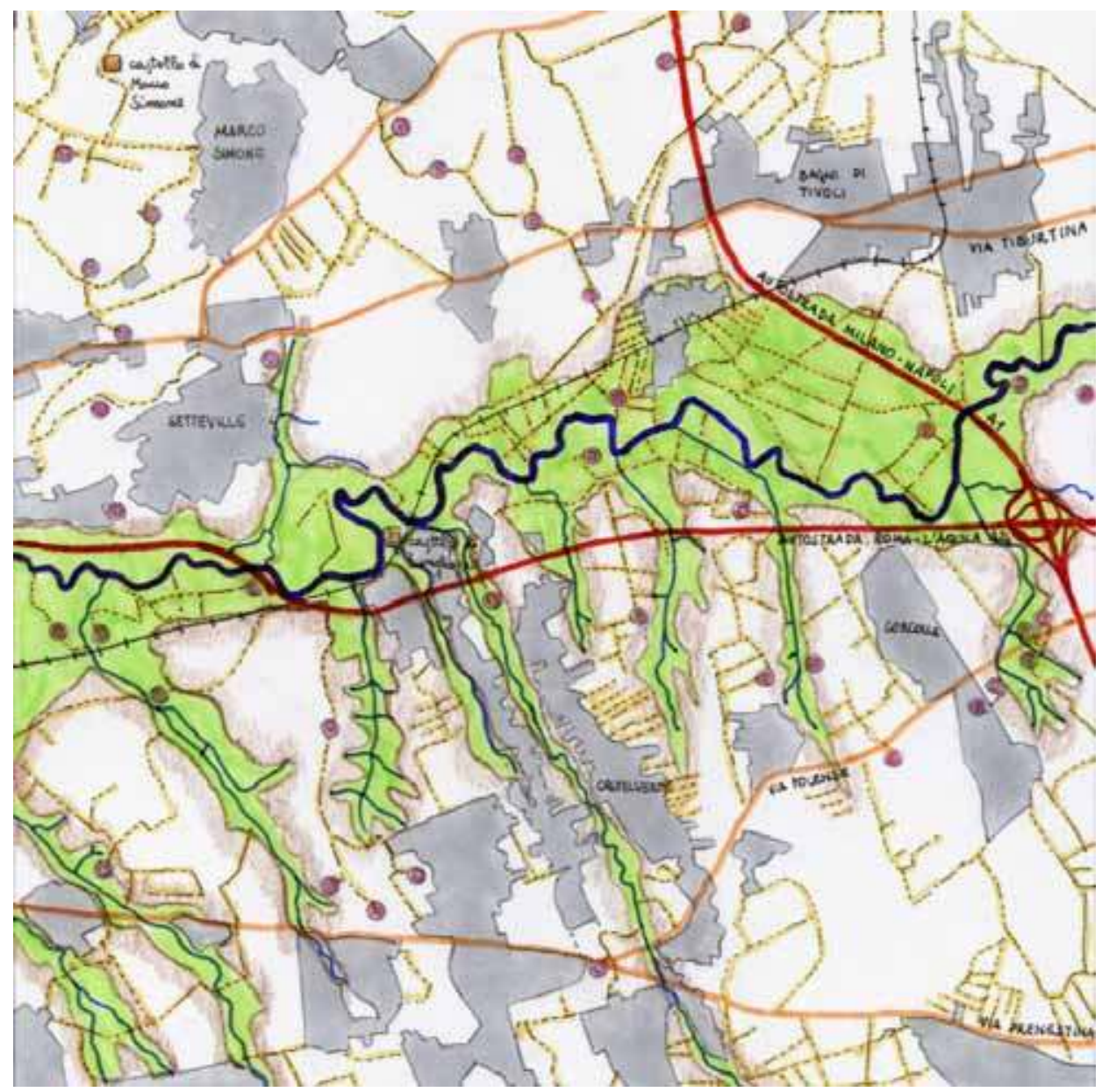

Figura 47. La "campagna" delle tenute del settore est: elementi componenti e relazioni. In blu è evidenziato il sistema delle acque; in rosso e arancio la viabilità principale; in giallo la viabilità interpoderale e con un pallino rosso le unità insediative agricole dei casali; in verde chiaro e marrone l'alternanza di valli e pianori; in grigio l'urbanizzato. 
Settore Sud: le tenute di DeCima, Monte Migliore, Radicelli E FALCOGNANA

Queste quattro tenute erano indicate da Pompeo Spinetti nella Carta dell'Agro Romano del 1913 come di proprietà delle famiglie dei Principi Pallavicini (tenuta di Decima), dei Colonna (tenuta di Monte Migliore), degli Angelici (tenuta di Radicelli) e deiBoncompagni Ludovisi (tenute di Falcognani).

Oggi parte di queste tenute è interessata dall'area comunale protetta di Decima - Malafede. Quest'area, compresa tra la via Pontina e la via Laurentina, è caratterizzata dalla presenza di beni geomorfologici e vegetazionali di notevole valore. Essa è connotata dal corso del fosso di Malafede e dai suoi affluenti, che disegnano il tipico paesaggio delle "forre".

\section{La morfologia e l'idrografia dei terreni}

Le tipologie di terreno esistenti sono legate alle fasi più recenti dell'evoluzione geologica dell'area che, fino a circa un milione di anni fa, era sepolta sotto le acque del mare.

Le formazioni affioranti vanno dalle più recenti di origine continentale, quali i depositi alluvionali presenti sul fondo dei fossi e delle marrane e le sabbie rossastre proprie dell'ambiente dunale, fino ai prodotti dell'attività vulcanica dei Colli Albani, rappresentati in prevalenza dalle diverse colate piroclastiche e lave. Scendendo nella successione, si hanno limi e sabbie gialle mescolate a ciottolini calcarei e silicei sparsi o concentrati e argille azzurre e grigie di ambiente lacustre.

Dal punto di vista idrogeologico l'area si estende su un vasto settore del margine occidentale dell'Unità idrogeologica dei Colli Albani, 
prossimo al Tevere ed al mare. Le falde acquifere hanno, quindi, grande continuità e spessore e sono particolarmente ricche, in quanto vengono ricaricate sia dalle precipitazioni, sia dai flussi sotterranei che provengono proprio dai settori interni dell'Unità idrogeologica albana.

L'idrografia del sistema è strutturata intorno al fosso di Malafede e ai suoi affluenti che, con un andamento parallelo prevalentemente in direzione est-ovest, incidono l'altopiano tracciando un disegno caratteristico, "a pettine".

Il differente grado di resistenza opposto dalla disuguale consistenza e durezza del mantello all'azione erosiva delle acque dà origine a due tipi di formazioni vallive:

- le prime, formate dal materiale tufaceo, presentano un carattere aspro ed accidentato con pareti sub-verticali e conservano, solitamente, impianti vegetali naturali;

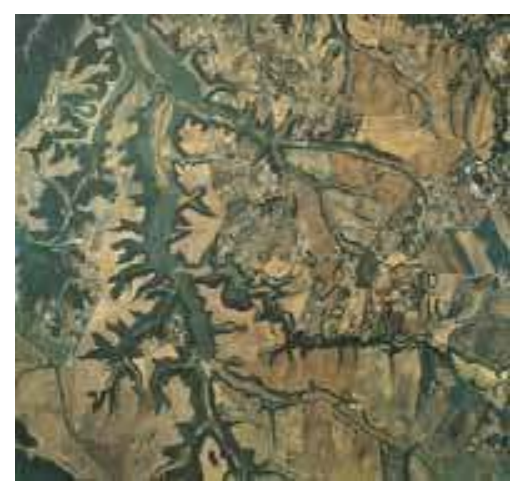

Figura 48. La morfologia dell'area è ben evidente dalle foto aeree.

- le seconde, formate dalle sabbie, hanno una forma dolce ed arrotondata e pendenze prive di brusche variazioni; in esse il manto vegetazionale naturale è stato intaccato e sostituito dalle coltivazioni agricole.

La morfologia della zona risulta, quindi, caratterizzata dalla presenza di un "plateau vulcanico" variamente inciso dall'azione erosiva del reticolo idrografico ad andamento dendritico. Sono dunque presenti ampi pianori che, essendo a loro volta modellati ed erosi dai processi dell'attività idrica e meteorica, si alternano con valli che presentano ampie scarpate e che mettono in risalto il limite tra i depositi vulcanici ed i sedimenti continentali - marini.

Nasce così un paesaggio disegnato da colline, con le sommità pianeggianti interrotte da valli strette o ampie. 


\section{I "residui" ambientali e/o storici}

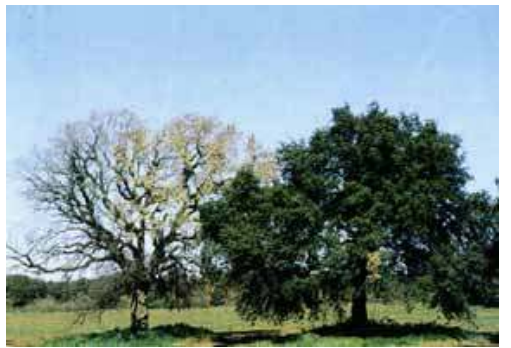

Figura 49. La Sugherata di Vallerano.

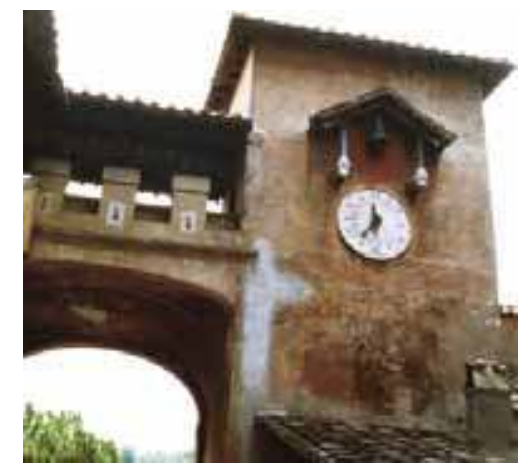

Figura 50. II castello di Decima.
L'area presenta una significativa diversità di complessi vegetazionali, raggruppati in ambiti molto vicini tra loro, dove convivono specie molto diverse che vanno dal leccio con la variante a sughera, al bosco mesofilo a cerro-farnetto, ad estesi lembi di vegetazione igrofila con pioppi, ontani, salici.

In particolare, il fosso di Malafede e i suoi affluenti di sinistra presentano pendici particolarmente ricche di vegetazione boschiva con continuità e con notevole pregio per densità ed estensione delle essenze; al contrario, di minore estensione e continuità sono le pendici degli affluenti di destra che, generalmente, conservano una migliore qualità vegetazionale nella porzione esposta a nord/nord-est ed un peggior stato del manto boschivo nei tratti esposti a sud/sud-ovest.

Di notevole interesse è l'antichissima Sugherata di Vallerano, un bosco di circa 100 ettari che ancora oggi sopravvive nella zona a ridosso della via Pontina.

La frequentazione umana di questo territorio è antichissima: insediamenti corrispondenti a varie fasi (dal Paleolitico all'Età del Bronzo) sono stati, infatti, ritrovati in diversi luoghi, fra i quali spicca la grande necropoli di Decima.

Altre testimonianze risalgono al periodo romano, quando il territorio venne occupato soprattutto da ville rustiche, e medievale, con il castello di Decima, la torre di Perna e numerosi resti ormai inglobati nelle strutture dei casali moderni.

Nel Rinascimento, infatti, la diminuita esigenza di difesa militare provocò la riconversione di castelli e torri in casali, che divennero il fulcro insediativo delle grandi tenute del ‘ 500 . 


\section{Le sistemazioni agrarie}

L'agricoltura è, anche in questa zona, l'attività più diffusa e svolge un importante ruolo di manutenzione del territorio e di presidio rispetto alla pressione urbana presente ai suoi margini.

I fondovalle, di media ampiezza, con la presenza della vegetazione igrofila ripariale lungo le sponde dei fossi, sono solitamente tenuti a seminativo nudo, che viene alternato ciclicamente agli erbai per il pascolo.

Nello stesso modo gli stretti altopiani, per larghi tratti mediamente modellati e compresi nel "pettine" dei corsi d'acqua, presentano una conduzione ancora a seminativo nudo con l'esclusione di alcune porzioni coltivate a frutteti e uliveti.

Gli appezzamenti dei terreni sono generalmente di ampie dimensioni ed il casale è solitamente posto al centro.

\section{L'insediamento rurale e la tipologia delle aziende e della loro conduzione}

La maggior parte dei numerosi casali esistenti oggi in questa porzione di territorio risale a strutture realizzate negli anni '30 su resti di edifici preesistenti di origine per lo più medievale, così come la rete dei percorsi di servizio, gli elementi e le trame agricole che strutturano l'attuale paesaggio agrario.

La tipologia insediativa agricola ricorrente in questa porzione di territorio è quella composta da grossi complessi rurali posti a distanze cadenzate e supportati da una serie di casali e manufatti minori sparsi

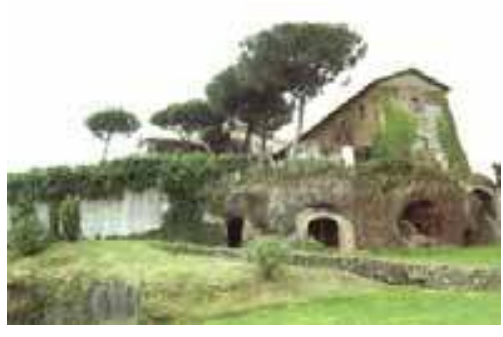

Figura 51. L'esempio di un casale più o meno moderno, "appoggiato" su preesistenze di diverse epoche storiche. nella tenuta.

Solitamente il casale della residenza è posto di fronte ad un ampio spiazzo intorno al quale si ritrovano anche numerosi altri edifici di 


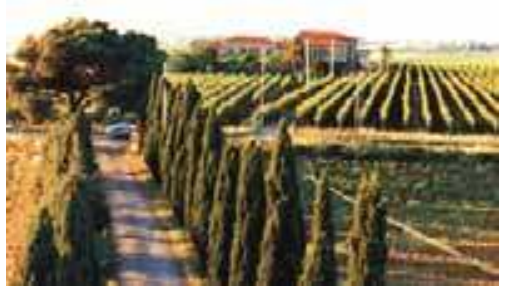

Figura 52. II casale della Falconiana in mezzo ai campi coltivati. servizio alla attività agricola. L'intero complesso è poi protetto da siepi e filari arborei e vi si accede tramite un viale alberato con cipressi o pini.

La viabilità interpoderale è particolarmente ricca, caratterizzata da lunghi assi in terra battuta che il più delle volte servono a collegare il casale principale con gli annessi agricoli sparsi nella tenuta e con le principali arterie di collegamento presenti nella zona.

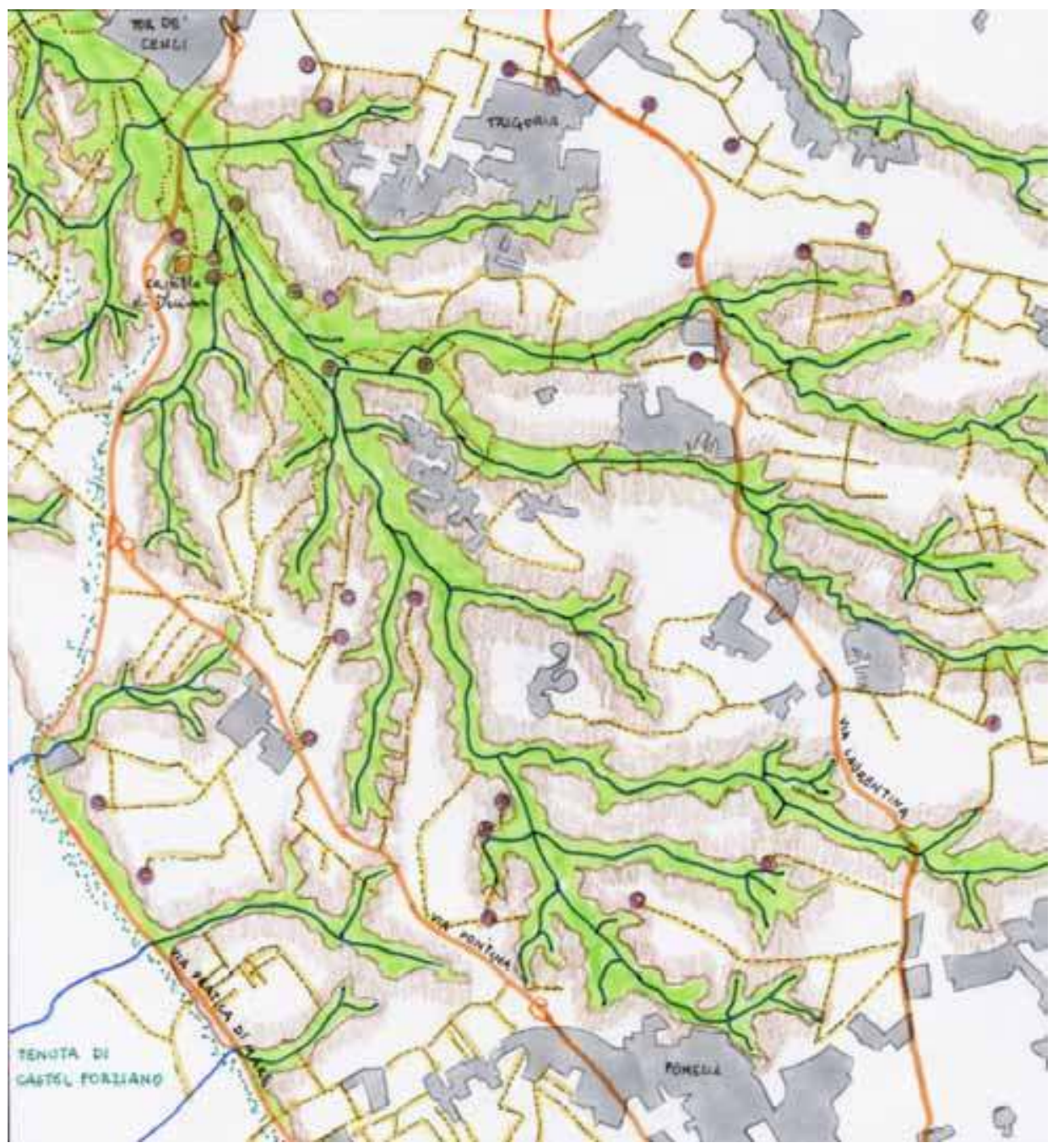

Figura 53. La "campagna" delle tenute del settore sud: elementi componenti e relazioni. In blu è evidenziato il sistema delle acque; in rosso e arancio la viabilità principale; in giallo la viabilità interpoderale e con un pallino rosso le unità insediative agricole dei casali; in verde chiaro e marrone l'alternanza di valli e pianori; in grigio l'urbanizzato. 


\section{LA CAMPAGNA DELLE BONIFICHE}

Le più importanti opere di bonifica sul litorale romano furono intraprese a seguito della prima legge organica di bonifica del $1882^{8}$ : nel 1884 fu bonificato lo stagno di Ostia, molto più tardi prese l'avvio la bonifica di Isola Sacra. La bonifica idraulica fu iniziata all'inizio del secolo, ma il risanamento integrale fu eseguito solo dal 1920 dall'Opera Nazionale Combattenti. La bonifica delle aree più a nord iniziò realmente nel 1926 e riguardò l'area caratterizzata dal grande stagno di Maccarese - sulla riva destra del Tevere - insieme allo stagno delle Pagliete - situato poco più a nord, a sinistra del fosso dei Tre Denari.

I suoli provenivano dall'esproprio, da parte dello Stato, dei terreni di proprietà delle famiglie nobiliari dei Rospigliosi e dei Torlonia; l'intera operazione fu gestita dalla Società Maccarese, appositamente istituita.

Dopo una lunga e complessa serie di passaggi, la Società è diventata una vera e propria azienda pubblica ed ha attraversato un fase di profonda trasformazione, tanto che gli ex mezzadri sono oggi proprietari di ampie parti dell'azienda.

Il paesaggio originario di questa zona venne dunque profondamente trasformato dalle opere della bonifica.

Il paesaggio di Maccarese quale era sino al 1925 (...) si presentava ancora come un paesaggio pressoché naturale, con aree prive di un costante deflusso verso uno scalo naturale o artificiale al mare, ma anche - al contrario - ricco di svariate pendenze e contropendenze, legate ad una molteplicità di vallecole più o meno ampie disposte in modo da creare un complesso di piccolissimi bacini chiusi e separati, nell'ambito dei bacini maggiori. ${ }^{9}$

8 Per una trattazione completa delle leggi sulla bonifica applicate al territorio costiero romano, cfr. il sesto capitolo "Cause ed effetti delle principali trasformazioni nel paesaggio della campagna romana".

9 Citazione tratta da P. RoccaseccA, Urbe, Suburbio e Agro: confini in movimento, dal sito digilander.libero.it/aperture/articoli/2.17.html 
La bonifica fu attuata attraverso l'installazione di $500 \mathrm{~km}$ di canali di drenaggio e quattro idrovore principali: l'idrovora di Maccarese, posta più a sud; l'idrovora delle Tre Cannelle e quella della Torre, in prossimità del basso corso del fosso Arrone; infine l'idrovora delle Pagliete, ubicata nel sito del vecchio stagno omonimo.

Le bonifiche hanno modificato radicalmente l'assetto morfologico e paesistico del litorale romano, sostituendo ai caratteri paesisticonaturalistici tipici della costa tirrenica (lagune, macchia mediterranea, dune) nuovi elementi antropici quali canali, filari alberati di vegetazione esotica, colture irrigue, strade, i quali, a conti fatti, hanno rappresentato il volano del successivo fenomeno dell'urbanizzazione ${ }^{10}$.

All'originario sistema insediativo agricolo, dunque, che si strutturava sulle grandi tenute agricole e su qualche casale sparso, è stato sovrapposto il sistema del nuovo insediamento agricolo, fatto di piccoli appezzamenti e dalla ricca rete dei casali di bonifica. Dal punto di vista paesistico-ambientale, alla macchia ed alle zone seminude, si sono in un primo momento sostituiti il grano - in quanto i terreni vergini non consentivano colture più esigenti - e, successivamente, le colture legnose (alberi da frutto e viti), gli ortaggi e, in qualche piccola zona, la floricoltura.

La zona è inoltre caratterizzata da una fitta presenza di viali frangivento di eucalipti, alcune volte sostituiti dai pini marittimi, che accanto ai casali ed alle fattorie più grandi si stringono a formare delle vere e proprie macchie arboree.

10 Sugli elementi strutturali del telaio regolare proprio della bonifica, infatti, nei decenni successivi si è andata via via appoggiando tutta una serie di lottizzazioni, più 0 meno regolari, localizzate - in una prima fase - a nastro lungo le arterie viarie principali, successivamente tramite una ricucitura quasi sempre reticolare lungo le infrastrutture secondarie. 


\section{La morfologia e l'idrografia dei terreni}

II territorio del litorale romano si suddivide in quattro zone che si presentano relativamente uniformi nei caratteri di idro-geologia, morfologia, suolo e vegetazione.

Iniziando dal mare, esse sono:

1. la spiaggia sabbiosa attuale: una fascia la cui larghezza oscilla dai 5 ai 10 metri, priva di vegetazione, in quanto l'azione del vento, che sposta in continuazione i granelli di sabbia, unita alle mareggiate, impedisce l'instaurarsi dei processi che portano alla formazione di un terreno vegetale.

2. Le dune recenti (dune litoranee, sabbie e riempimenti interdunari, dune costiere consolidate, dune interne): questo complesso geomorfologico si espande per una striscia di terreno formata prevalentemente da sabbie eoliche grigio-giallastre, fini, con minerali vulcanici. II sistema che si viene a formare, a seguito proprio dell'azione del vento, è una serie di cordoni dunali paralleli alla

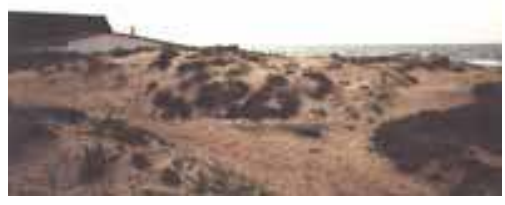

Figura 54. La duna recente. costa, più o meno consolidati dalla vegetazione, che possono innalzarsi fino a superare anche i 15 metri di altezza. Fra un cordone e l'altro vi sono depressioni - denominate lame - che vengono riempite dalle acque meteoriche e che contengono modesti depositi palustri e lagunari costituiti da limi, argille e torbe.

Per quanto riguarda i suoli, il primo cordone delle dune litoranee, che è ancora particolarmente colpito dalla continua ed insistente azione dei venti marini, ospita una scarsa vegetazione caratteristica delle zone aride, mentre nei successivi cordoni dunali le migliori condizioni ambientali hanno permesso l'instaurarsi della macchia mediterranea. In alcune lame interdunali, soprattutto là dove la falda freatica è più superficiale, è possibile ritrovare associazioni di pioppo, frassino minore e farnia. 
3. La duna antica: una fascia costituita da sabbie quarzose rossastre, di origine eolica, più o meno miste ad argille, che si estende dal limite delle dune recenti per circa $7,5 \mathrm{~km}$ all'interno, fino ad entrare in contatto con i depositi piroclastici del Vulcano Laziale.

La morfologia originaria era costituita da una serie di cordoni di dune continentali; col tempo l'azione di agenti esogeni ha in parte spianato le forme del terreno primitive, per cui attualmente ci si trova di fronte ad una morfologia sub-pianeggiante nella parte bassa della duna e ad una blanda ondulazione nella parte più alta ed interna.

Questa formazione presenta solitamente una discreta permeabilità, dovuta prevalentemente alla sua componente sabbiosa ed alla presenza di una serie di falde acquifere, più o meno indipendenti fra loro, che si manifestano con alcune sorgenti di modesta portata. Sono inoltre presenti numerose depressioni interdunali - denominate piscine - con un fondo poco permeabile, nelle quali si raccoglie l'acqua meteorica.

Sulla duna antica si trova attualmente una concentrazione di vegetazione particolarmente adatta alle condizioni ambientali umide od intermedie, formata da essenze quali il farnetto, la farnia, il cerro, e il pioppo ed il salice nelle zone più umide. Questa formazione vegetazionale è oggi alquanto degradata soprattutto a causa di un'antropizzazione secolare, che ha inserito numerosi elementi impropri di macchia mediterranea.

4. Le alluvioni recenti: formazioni geomorfologiche particolarmente permeabili, che affiorano nei fondovalle della rete idrografica principale e secondaria e sono costituite da banchi di ciottolate, argille, limi e sabbie.

Sono zone soggette ad un'agricoltura intensiva, alternata laddove i terreni presentano una conformazione più grossolana e sono più permeabili alla presenza di boschetti di pioppi. 


\section{I "residui" ambientali e/o storici}

Dal punto di vista ambientale, questa porzione di territorio è ricca dei cospicui "residui" dello spettacolare impianto naturale, rimanenze della copertura di vegetazione che in passato, senza alcuna soluzione di continuità, dal piede dei colli appenninici si estendeva sino al mare.

I maggiori esempi sono rappresentati dalla pineta di Fregene, dall'oasi di Macchia Grande, dalla pineta di Castelfusano, dalla tenuta presidenziale di Castel Porziano e dalla tenuta privata di Capocotta.

In queste aree sono presenti le quattro categorie della vegetazione tipica del litorale romano sommariamente elencate in precedenza: la vegetazione mobile delle sabbie, quella igrofila delle dune, la macchia mediterranea, il querceto termofilo.

Dal punto di vista dei "residui" storici è opportuno ricordare come l'intera zona, essendo stata oggetto della bonifica all'inizio del XXI secolo, sia stata completamente riconfigurata e abbia perso gran parte dei suoi caratteri storici originari.

Nonostante ciò, sono presenti notevoli resti di archeologia romana e di permanenze legate alla storia medievale: bisogna segnalare almeno l'area archeologica di Ostia Antica, situata lungo la via Ostiense in prossimità di una delle ultime anse della foce del Tevere, e la necropoli di Porto, vicino all'agglomerato urbano di Isola Sacra, a testimonianza del periodo romano; il castello di Maccarese e la torre di Palidoro a nord e la torre di Tor Paterno a sud, simboli dell'antico sistema insediativo medievale imperniato sui cosiddetti centri di arroccamento; i numerosi ritrovamenti avvenuti entro la tenuta presidenziale di Castel Porziano ed in quella privata di Capocotta.

Alcune permanenze sono, inoltre, ancora visibili nelle strutture edificate di casali che hanno inglobato al proprio interno i resti delle torri medievali che caratterizzavano quest'area e ne rappresentavano l'avamposto militare difensivo lungo la costa.

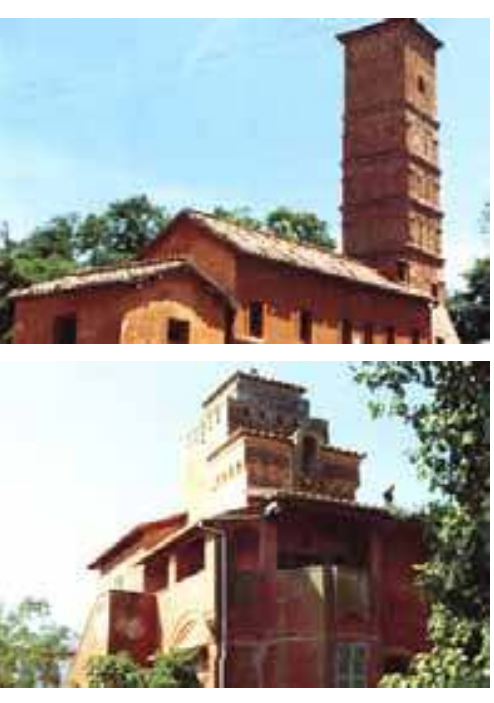

Figure 55-56. Alcuni esempi di casali entro i quali sono ancora rintracciabili i "residui" storici di costruzioni militari e religiose. 
Esistono esempi similari anche per ciò che riguarda i resti di antichi edifici religiosi, che sono stati anch'essi inglobati nelle strutture edilizie più recenti (anni '20 e anni '30) dei casali di bonifica.

\section{Le sistemazioni agrarie}

II risultato più evidente della bonifica, ovviamente oltre al prosciugamento delle paludi, è rappresentato da una vasta rete di canalizzazioni e vasche di espansione.

L'organizzazione agraria del territorio a nord ed a sud della foce del Tevere dimostra di avere, nonostante entrambe le parti presentino questa fitta rete di canali, caratteristiche strutturali diverse.

L'area a nord del fiume, infatti, ha una maglia fitta e regolare di canali, collettori e colatori tessuta su una rete di strade principali e poderali lungo le quali si attestano le colonie agricole. Queste ultime hanno per lo più dimensioni che variano fra 20, 25 e 40 ettari, con lotti pressoché regolari di forma rettangolare. La coltura prevalente è quella della carota, alla quale si accompagnano le coltivazioni di grano e foraggio, i vigneti ed i frutteti, le serre ed i vivai e l'allevamento di bovini da latte.

L'area a sud del fiume, invece, è costituita da una struttura imperniata sull'incrocio dei due collettori primari, dai quali si dirama l'insieme dei canali secondari che seguono l'andamento un po' più tortuoso del Tevere, originando una sorta di orditura concentrica bordata da alberature frangivento, meno fitta della precedente. Questo tipo di struttura è evidenziata dalla maglia viaria, lungo la quale si trovano $\mathrm{i}$ casali ed i centri agricoli.

La forma dei lotti, al contrario di quanto avviene per gli appezzamenti a nord, risulta varia, rettangolare, trapezoidale o triangolare, proprio a causa della forte influenza che, in questa zona, ha la fisionomia del 
corso fluviale. L'attività agricola è costituita prevalentemente da seminativo irriguo ed è affiancata da una consistente attività zootecnica, con numerosi allevamenti di cavalli.

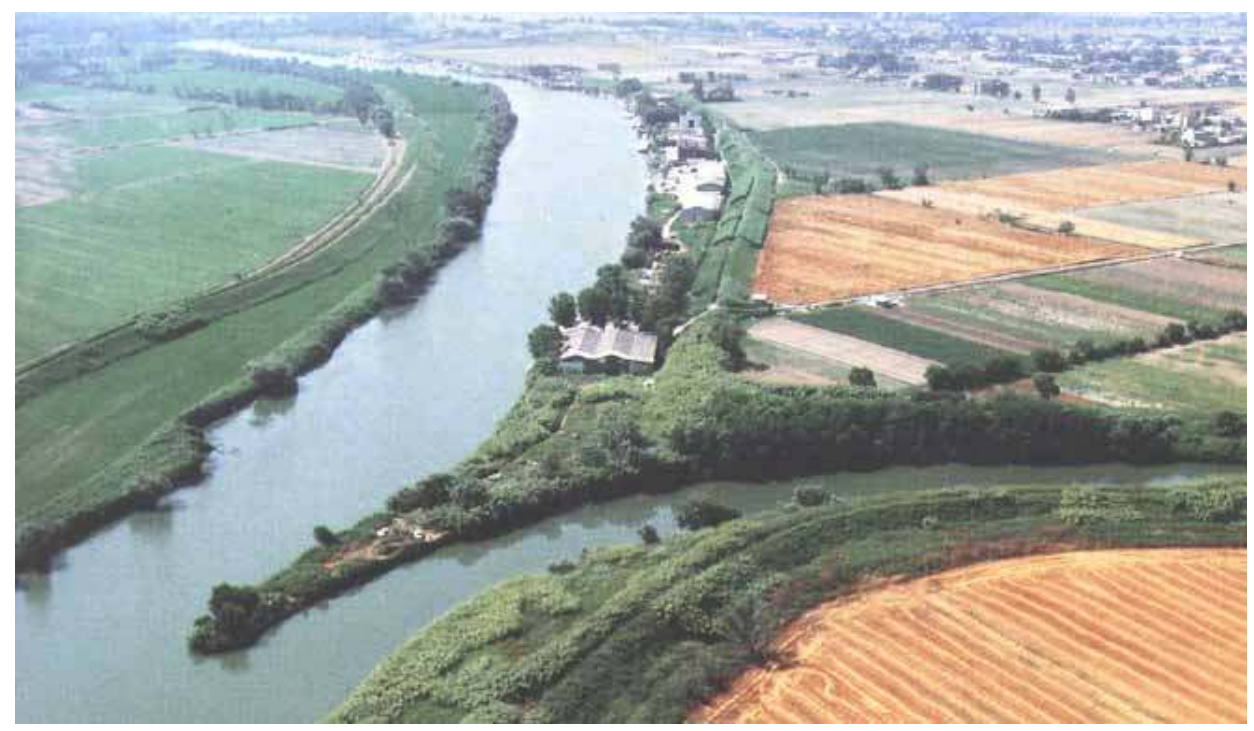

Figura 57. La biforcazione del Tevere guardando verso il mare: è evidente la diversa tessitura agraria lungo le due sponde del fiume.

\section{L'insediamento rurale e la tipologia delle aziende e della loro conduzione}

Come già detto, il sistema organizzativo di questa "campagna" è riconducibile ad un'orditura a maglia le cui componenti, pur presentando variazioni di dimensioni ed orientamento, seguono una gerarchia ben riconoscibile.

L'asse portante di questo sistema è composto dall'insieme formato dal canale di bonifica e dalla strada principale, limitati ai bordi da alberature frangivento. La sezione dell'asse secondario ha la strada o il canale come elemento portante, sempre affiancati su entrambi i lati dall'alberatura di eucalipti o pini marittimi. L'asse elementare di ricucitura fra i lotti, infine, prevede sempre il canale come elemento portante,

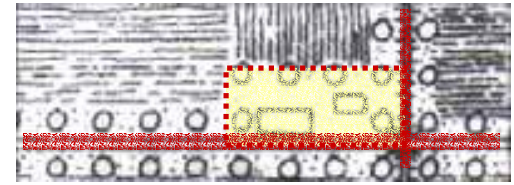

Figura 58. Unità insediativa agricola all'incrocio di due assi generatori.

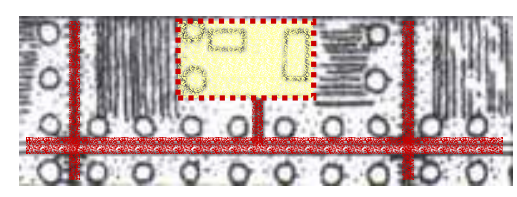

Figura 59. Unità insediativa agricola interna al lotto. 

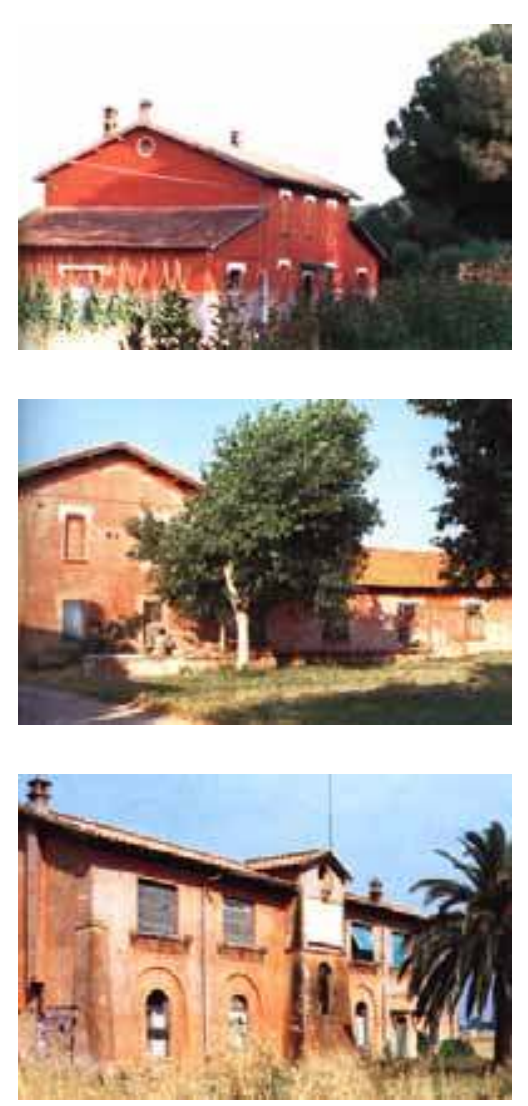

Figure 60-62. Alcuni esempi di casali tipici della bonifica situati a Maccarese, Fiumicino e Isola Sacra. accompagnato in alcuni casi dall'alberatura frangivento lungo uno dei suoi bordi.

L'unità insediativa agricola rappresentata dal casale di bonifica, a secondo della sua posizione rispetto agli assi appena descritti, dà luogo ad alcune relazioni tipo che è possibile individuare come ricorrenti in questa porzione di campagna romana.

Una prima tipologia è quella contraddistinta da un'unità insediativa agricola posizionata all'incrocio di due assi generatori: asse principale e asse secondario, oppure asse secondario e asse di ricucitura.

La seconda tipologia ricorrente è simile alla precedente, ma se ne differenzia in quanto in questo caso il casale, con gli annessi agricoli, è posizionato tangenzialmente rispetto ad un solo asse generatore.

La terza tipologia, probabilmente la più consueta, è quella che ha l'intera struttura insediativa collocata internamente al lotto, in maniera abbastanza baricentrica.

La quarta ed ultima tipologia è quella che è più facile rintracciare soprattutto nella zona a sud della foce ed è caratterizzata dall'unità insediativa agricola posizionata senza un orientamento ben preciso all'interno del lotto agricolo.

II casale della bonifica presenta una struttura ricorrente, basata su una serie di edifici - tutti di un color rosso mattone - articolati intorno ad un cortile e costituita da un edificio centrale adibito alla residenza, composto da più corpi sfalsati secondo una planimetria $a d \mathrm{~L}$, e da altri edifici minori e di servizio, adibiti il più delle volte a stalle e a granai. II cortile viene protetto dalla viabilità di accesso da una fitta schermatura verde di pini e cipressi.

Dal punto di vista della proprietà, infine, buona parte dei terreni a nord della foce è oggi di proprietà della SOGEA S.p.A., la quale svolge un'attività produttiva che la vede primeggiare in tutta Europa per la raccolta delle carote. 
In anni più recenti la Società ha avviato un processo di frazionamento delle terre di proprietà, attraverso una ripartizione poderale dei terreni agricoli che vengono ceduti direttamente ai mezzadri come liquidazione. Questo processo, al momento, non ha ancora portato a grosse modifiche dell'uso agricolo dell'area, ma va tenuto conto come un frazionamento di questo tipo possa rappresentare un facile bersaglio tanto per pressioni legate a progetti di trasformazione, tanto per eventuali tendenze all'abbandono dell'attività agricola.

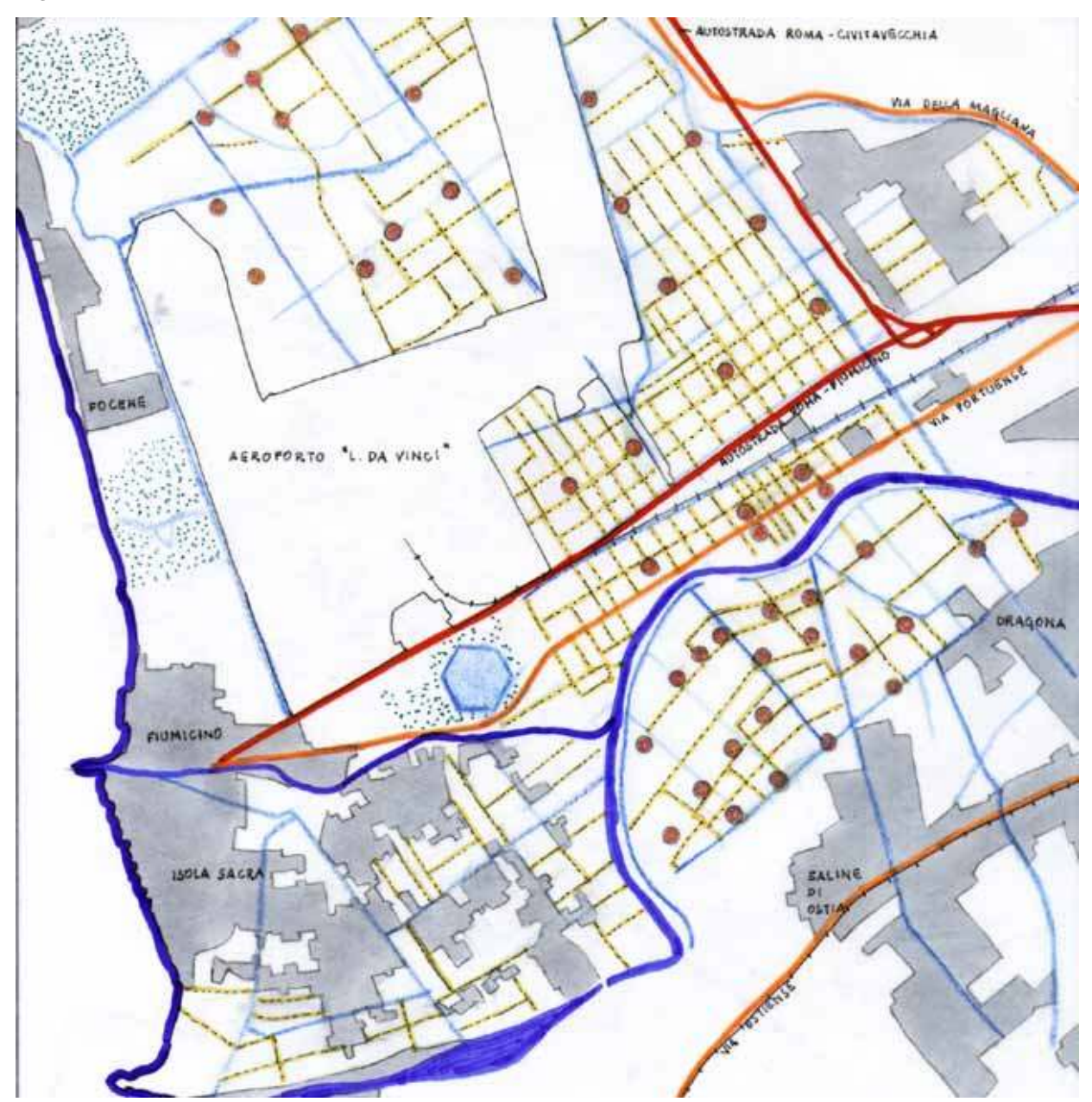

Figura 63. La "campagna" della bonifica: elementi componenti e relazioni. In blu e azzurro è evidenziato il sistema delle acque con la rete dei canali e dei fossi della bonifica; in rosso e arancio la viabilità principale; in giallo la viabilità interpoderale e con un pallino rosso le unità insediative agricole dei casali; in verde puntinato le pinete e le macchie mediterranee residue; in grigio l'urbanizzato. 


\section{LA CAMPAGNA DEL CITTÀ METROPOLITANA}

Per «campagna della città metropolitana» si intendono quelle ampie porzioni di territorio dell'area romana nelle quali, dagli anni '60 in poi, le strutture tradizionali del paesaggio agrario sono state via via modificate ${ }^{11}$ - talvolta compromesse irrimediabilmente - dalla presenza di nuovi nuclei edilizi e lottizzazioni.

La localizzazione sul territorio di questi insediamenti, pur essendo nella maggior parte dei casi abusiva, non è avvenuta in modo del tutto casuale, bensì a seguito di regole dettate dalle necessità abitative. Dalle letture dello stato di fatto che sono state svolte in studi più o meno recenti risulta evidente che vi si possono ritrovare alcune situazioni tipo.

Una prima tipologia di insediamenti è rappresentata da quelli sorti lungo le principali infrastrutture viarie e ferroviarie, che sono diventate vere e proprie direttrici di sviluppo, rappresentando rapidi ed economici mezzi di collegamento con il centro direzionale urbano. Questo tipo di insediamenti è scaturito essenzialmente da una stato di necessità abitativa e, quindi, è stato quasi sempre destinato agli strati sociali più poveri.

Allontanandosi da queste direttrici, le costruzioni perdono la loro caratteristica peculiare di bene di necessità per diventare, in alcuni casi, veri e propri centri residenziali con tipologie e caratteristiche medioeconomiche o addirittura di lusso.

Un terzo esempio è rappresentato dalle situazioni insediative che hanno interessato la zona costiera, dove le iniziative speculative per le seconde case sono state accompagnate dagli insediamenti sorti spontaneamente anche a causa della vicinanza delle attività legate alla presenza del mare, dell'aeroporto o dell'azienda agricola di Maccarese.

11 Cfr. ancora il sesto capitolo "Cause ed effetti delle principali trasformazioni nel paesaggio della campagna romana”. 
Sono così presenti oggi nella campagna romana diverse situazioni nelle quali l'innesto di un nuovo elemento qual è quello dell'edilizia spontanea ha dato origine a nuove relazioni con i caratteri del paesaggio agrario preesistente. I criteri di lettura sin qui adottati, quindi, devono in qualche modo evolversi, introducendo fra gli elementi strutturanti il paesaggio, oltre alla morfologia dei luoghi, alle preesistenze vegetazionali e/o storiche ed alle coltivazioni agrarie, anche la tipologia e la conformazione degli insediamenti.

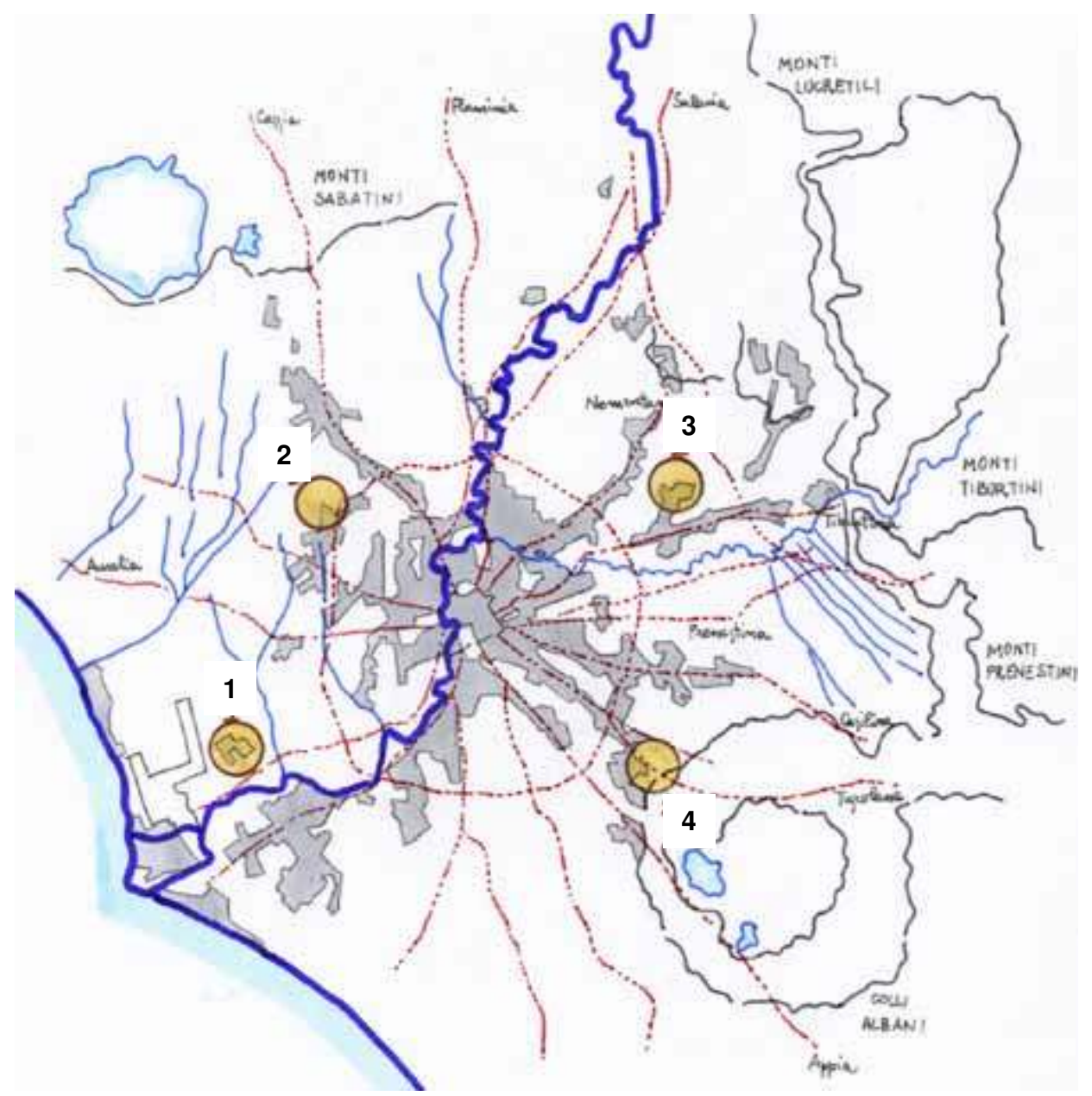

Figura 64. La localizzazione nell'area romana dei casi analizzati per la campagna della città metropolitana: 1. Piana del Sole, 2. Lucchina, 3. Marco Simone, 4. Casal Morena.

Nel territorio romano abbiamo individuato e illustrato alcune di queste nuove realtà: per ciascuna, identificati gli elementi componenti, si 
è valutato fino a che punto il tessuto insediativo abbia aderito alle matrici storiche preesistenti e che ruolo assuma oggi in questi contesti la connotazione agricola, delineando infine le linee di struttura di questi "nuovi" paesaggi.

È possibile anticipare alcune situazioni significative:

- una situazione in cui il rapporto tra l'edificato e la struttura del paesaggio preesistente è ben riconoscibile e compiuto, tanto che si può dire che ci si trova di fronte a veri e propri nuovi quadri paesaggistici, e il ruolo dell'attività agricola ha ancora un ruolo definito; benchè spesso localizzato ai margini;

- una situazione nella quale i rapporti tra i vari elementi - nuovi o preesistenti che siano - sono presenti, ma non sono chiaramente o completamente strutturati, così come avviene per l'attività agricola che rappresenta solo una serie di spazi aperti ancora suscettibili ei edificazione;

- un'ulteriore situazione che vede un rapporto di completa indifferenza tra i nuovi inserimenti e le componenti preesistenti.

\section{Settore sud-ovest: Piana del Sole}

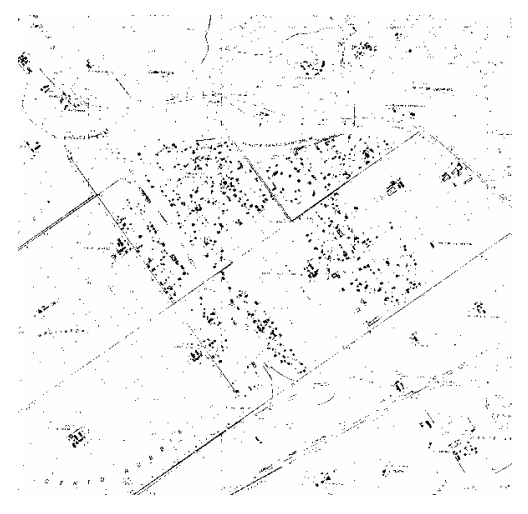

Figura 65. La situazione dell'agglomerato di Piana del Sole nel 1991.
II nucleo denominato Piana del Sole è localizzato in una porzione di territorio posta a sud-ovest di Roma ed è compreso tra i tre grandi assi infrastrutturali di interesse territoriale delle autostrade Roma-Fiumicino e Roma-Civitavecchia e della ferrovia Roma-Pisa.

L'insediamento di Piana del Sole presenta essenzialmente un tessuto che si è sviluppato secondo il modello reticolare generato dagli elementi propri della bonifica; solo in alcuni punti si è già totalmente richiuso su stesso attraverso la definizione di margini certi dell'edificato e la saturazione delle aree ancora libere presenti al suo interno. 
II tipo edilizio più ricorrente è il villino mono o plurifamiliare, composto da tre piani, con annesso il giardino.

L'area è caratterizzata da una morfologia pianeggiante e monotona, con quote che non superano i 5 metri e un terreno prevalentemente argilloso ed in parte morboso, solcato dalla fitta rete dei canali della bonifica di Maccarese.

Questi elementi rappresentano, come abbiamo già avuto modo di vedere, il sistema strutturante l'intera zona e costituiscono un patrimonio culturale ancora efficiente. Inoltre, le canalizzazioni della bonifica e le alberature ad esse associate rappresentano, soprattutto nella zona più vicina all'urbanizzato, gli unici "elementi naturali" che legano, dal punto di vista vegetazionale, la costa all'entroterra dei terrazzi costieri.

L'attività agricola si presenta differentemente a seconda che essa si svolga negli spazi interclusi all'edificato o nelle zone esterne.

Nel primo caso i lotti sono coltivati ad orto, per lo più per un consumo familiare, e, pur mantenendo la regolarità dovuta alle opere di bonifica, non è presente nessun elemento di connessione tra questi e gli edifici che li circondano.

Nel secondo caso, invece, il paesaggio agrario è quello già descritto per la «campagna della bonifica», dove i campi di media estensione sono separati tra loro dai canali e dalla viabilità interpoderale e dove è ancora ben evidente la regolarità delle unità insediative dei casali disegnata con le opere della bonifica idraulica.

In questo contesto, che si potrebbe definire quasi urbano, è indubbio che il rapporto con l'intorno (masse, volumi, distacchi) sia meno traumatico che nelle zone dell'edilizia ad alta densità. È però altrettanto vero che il danno maggiore si ha per l'enorme consumo di suolo attuato a danno dell'uso agricolo, per l'alterazione delle risorse paesistiche ed ambientali che rappresentavano la trama e la regola storica propria di 
questi luoghi, per il forte inquinamento delle falde idriche e per il disegno visivo provocato da questo "paesaggio urbano" monotono e desolante.

La maglia insediativa reticolare di Piana del Sole ha, infatti, quasi completamente avvolto alcuni brandelli residui dell'agro romano, trasformandoli di fatto in zone in cui il degrado degli elementi della struttura storica della bonifica è praticamente irreversibile. Tutto ciò ha comportato un graduale ma progressivo abbandono dell'attività agricola che, conseguentemente, coinvolge alcune testimonianze storiche come $\mathrm{i}$ casali, le vaccherie, i centri agricoli che, talvolta, è ancora possibile rintracciare annegati nel tessuto insediativo urbano.

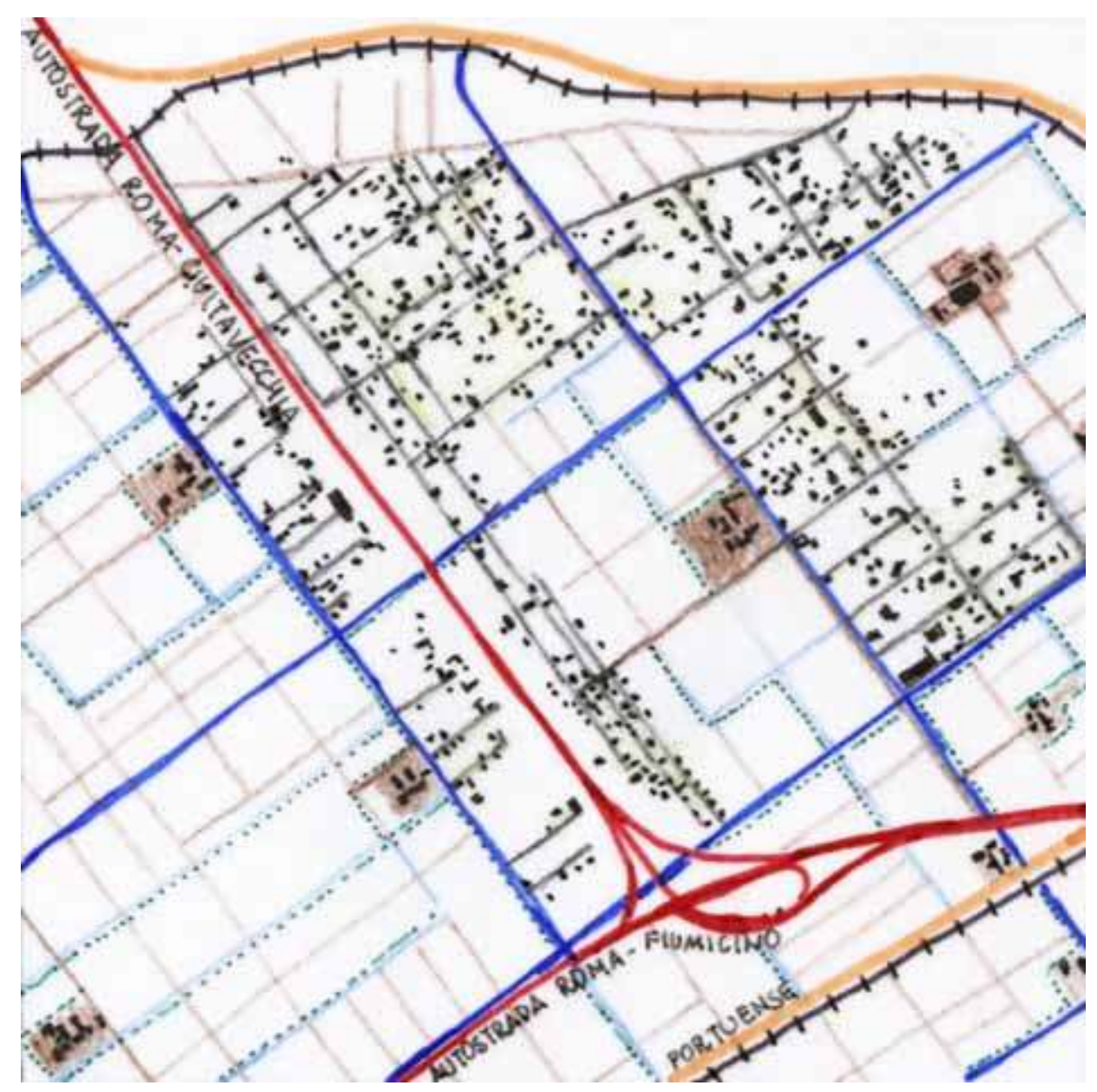

Figura 66. II rapporto tra edificato e paesaggio agrario è pressoché inesistente, se non nella regolarità della maglia della bonifica seguita anche dall'insediamento. 
Il rapporto tra l'urbanizzato e l'insieme degli elementi componenti il paesaggio presenta dunque due facce: se da un lato la regola delle canalizzazioni della bonifica è stata di fatto la regola con la quale si è strutturato l'insediamento di Piana del Sole, dall'altro lato non vi è oggi quasi alcun rapporto morfologico o funzionale tra l'edificato e le aree agricole circostanti od intercluse ad esso.

L'uso agricolo - sia quello intercluso, sia quello a margine dovrebbe, quindi, in questo caso essere inteso come una sorta di avamposto, di zona cuscinetto, dove è possibile riscoprire le tracce storiche dei luoghi e con esse la loro identità specifica.

Se è vero che l'agricoltura ha modificato l'aspetto originario di questo territorio, bisogna ammettere che si è trattato dell'intervento antropico meno devastante: la sua presenza, in questo territorio, costituisce un valore di memoria storica, oltre che produttivo e sociale (...). ${ }^{12}$

Contemporaneamente queste porzioni di territorio agricolo rappresentano gli elementi fondamentali di una rete ecologica che, oggi, è in gran parte stata "strappata" dalla presenza dell'insediamento.

\section{SETTORE NORD-OVEST: LUCCHINA}

L'edificato della Lucchina è localizzato nella parte nord di Roma, a diretto contatto con il Grande Raccordo Anulare e nelle immediate vicinanze della Borgata Ottavia. II territorio circostante non è stato particolarmente interessato da un'urbanizzazione diffusa.

La ragione è probabilmente da rintracciare tanto nell'andamento orografico del terreno, che di per sé non ha permesso un'agevole edificazione, quanto nella presenza di un'agricoltura particolarmente

12 M. Bertollini, A. Parisi Presicce, Da periferia a città, in C. Bagnasco (a cura di), «ll delta del Tevere: un viaggio fra passato e futuro», F.lli Palombi, Roma 1998, p.131. 


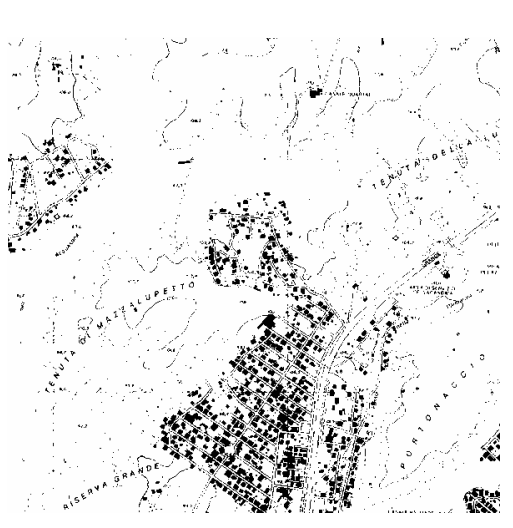

Figura 67. Lucchina nel 1991.

remunerativa e nella struttura della proprietà fondiaria (la maggior parte dei terreni erano di proprietà di ordini religiosi).

Dal punto di vista prettamente geologico, questa porzione di territorio è parte integrante di una più vasta area caratterizzata da una quasi esclusiva copertura eruttiva dovuta all'attività dell'apparato vulcanico Sabatino. La natura vulcanica dei terreni condiziona fortemente l'andamento orografico della zona: il carattere detritico dei corsi d'acqua e il processo di erosione e modellazione dei suoli vulcanici, infatti, danno luogo ad una morfologia caratterizzata dalla presenza di pendici collinari vere e proprie e di "forre" alternate ad altopiani.

Le coperture a bosco sono oggi generalmente conservate, in maniera massiccia e compatta, lungo le pendici vallive più esasperate $\mathrm{e}$ nelle morfologie torrentizie più anguste.

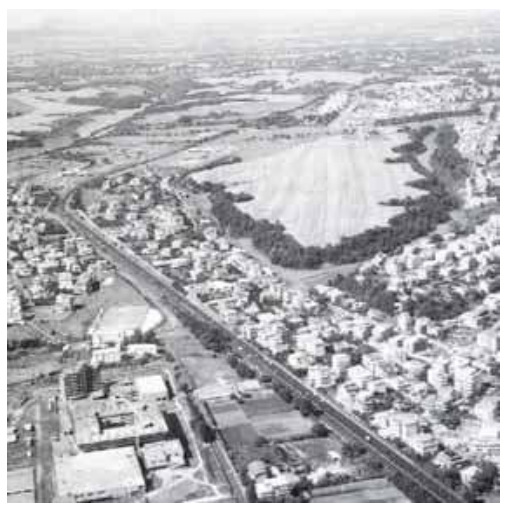

II rapporto che sussiste con il paesaggio, in questo caso, si presenta abbastanza compiuto, ovvero l'intero complesso urbano formato dalla struttura viaria e dal tessuto edilizio si è conformato ai caratteri naturali e paesistici del luogo.

L'andamento orografico ha condizionato sin dal passato gli insediamenti di tutta la zona: i maggiori assi stradali si sono sempre posizionati sulle dorsali collinari, dando origine ad una serie di percorsi

Figura 68. L'edificato è ben delimitato dagli elementi paesistici del contesto circostante. di crinale che hanno di fatto rappresentato gli assi strutturanti l'edificazione.

La forma complessiva dell'edificato della Lucchina è stata anch'essa definita dalla forma fisica del supporto orografico e la maglia viaria ha mantenuto con esso un rapporto coerente. II tessuto edilizio, che si estende sulla cima di un pianoro, è quasi completamente saturo ed è caratterizzato da palazzine di tre o quattro piani che seguono una struttura a pettine appoggiata su un asse stradale di crinale con un andamento nord-sud. 


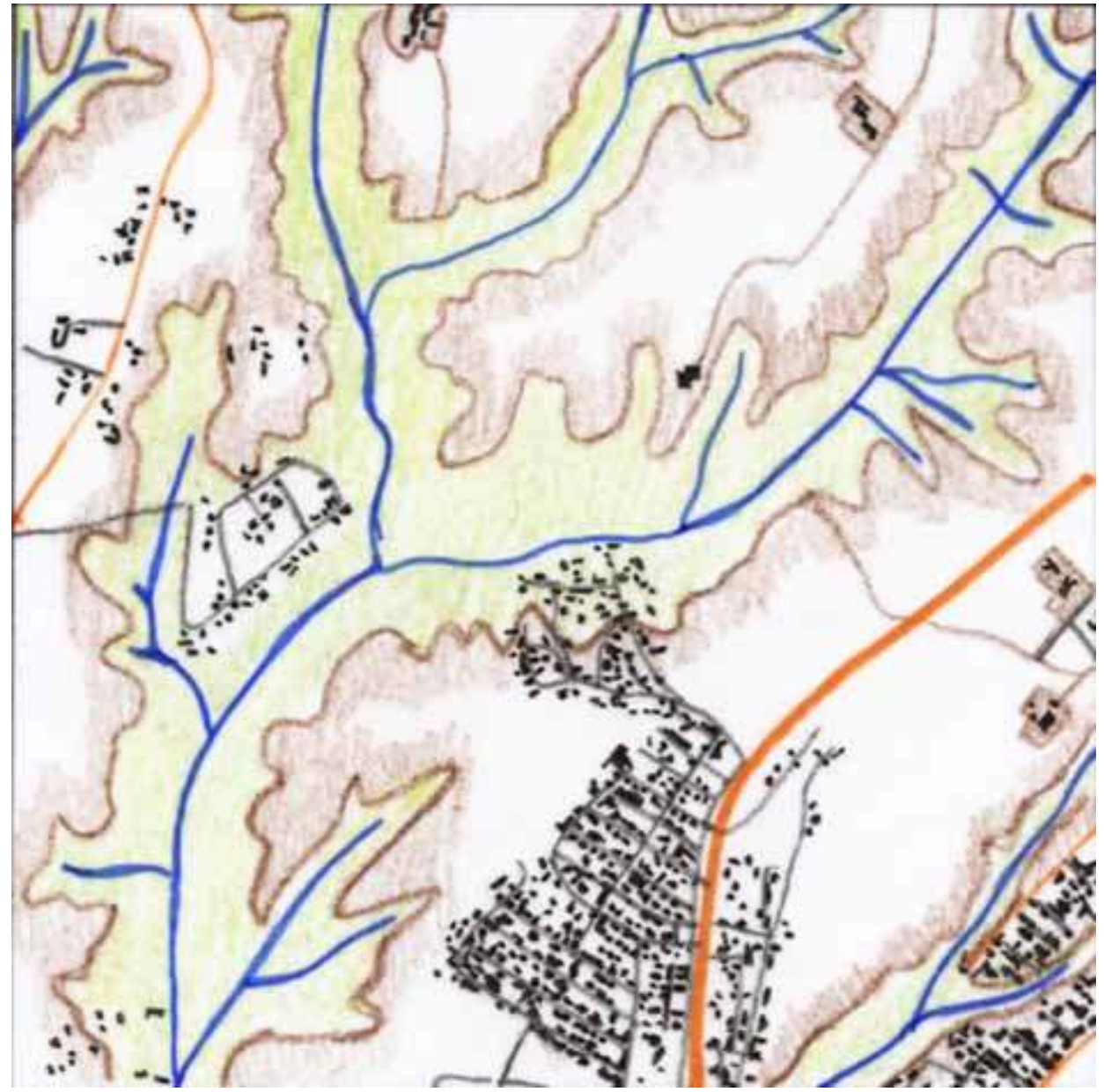

Figura 69. II rapporto tra edificato e paesaggio agrario è compiuto e l'edificato segue la regola morfologica dei luoghi.

In questo contesto territoriale l'attività agricola è quasi assente all'interno del contesto urbano. Al contrario, la zona circostante è interessata dalla presenza di numerose tenute nelle quali si svolge un'intensa attività agricola, particolarmente importante sia sotto il profilo meramente produttivo, sia sotto quello paesistico ambientale.

La presenza di queste aree agricole così a ridosso dell'urbanizzato svolge al contempo due ruoli fondamentali: da una parte il paesaggio agrario disegna il margine - sia dal punto di vista visivo, che da quello morfologico - della zona edificata, impedendo, di fatto, che questa si espanda a macchia d'olio nel territorio. Dall'altra parte le attività agricole 


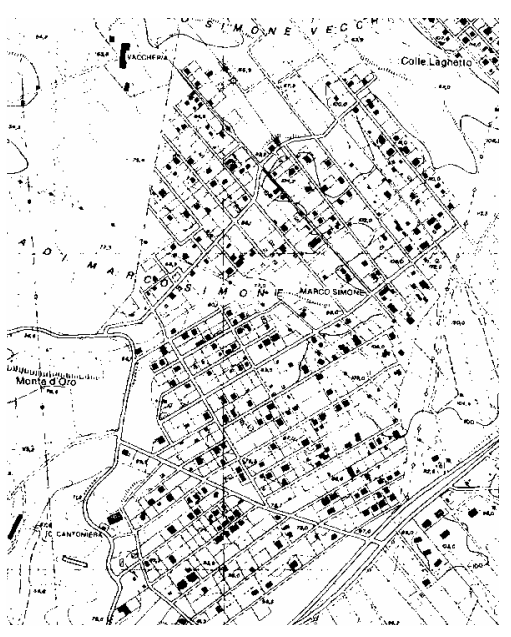

Figura 70. L'area di Marco Simone nel 1991. svolgono il ruolo sociale e culturale di memoria della trama storica del paesaggio agrario delle tenute, caratterizzato dalle colture estensive a rotazione tipiche dell'agro romano e dalla struttura insediativa dei casali posizionati lungo i percorsi di crinale sui pianori.

\section{SETTORE NORD-EST: MARCO SIMONE}

L'area esaminata consiste in una serie di lottizzazioni lungo la via Tiburtina, oltre il Grande Raccordo Anulare.

Tutto il paesaggio del settore est dell'agro è stato interessato sin dagli anni '60 da una forte trasformazione, con la realizzazione di numerosi insediamenti urbani che si sono configurati come un vero e proprio proseguimento della periferia romana.

La morfologia del territorio che si estende intorno a quella che era la tenuta di Marco Simone consiste in ampi altopiani che sono incisi solo superficialmente da alcuni fossi affluenti del fiume Aniene.

La vegetazione naturale risulta limitata a quella golenale, mentre la copertura dominante è a seminativo nudo.

II paesaggio risulta quindi caratterizzato da ampie visuali che episodicamente vengono interrotte dalla presenza di casali agricoli, gruppi di alberi o filari presenti lungo i percorsi di attraversamento.

Da una situazione iniziale caratterizzata, come detto, da insediamenti sparsi, case padronali e aziende agricole legate alla struttura della media e grande proprietà fondiaria, si è passati ad un progressivo processo di parcellizzazione dei terreni e di edificazione indiscriminata.

La zona è oggi interessata da un'edificazione diffusa, con larghi spazi ancora non costruiti, con una molteplicità di tipi edilizi ripetuti su lotti di piccole dimensioni, con una rete viaria il più delle volte disordinata e senza alcun livello gerarchico. Lo schema planimetrico è abbastanza 
regolare ed è formato da lotti di taglio pressoché uniforme derivanti dalle operazioni di lottizzazione dettate da una speculazione incentrata esclusivamente sulla vendita dei terreni.

Le tipologie più diffuse sono quelle della casa media e della palazzina con una densità media e con caratteristiche edilizie molto simili a quelle dell'urbanizzazione regolare.

L'attività agricola si svolge prevalentemente ai margini dell'edificato ed è prevalentemente caratterizzata da un'agricoltura superstite, inframmezzata da usi non agricoli (il golf club ne è un esempio) o da spazi liberi utilizzati come "verde urbano".

Solo in alcuni tratti sono ancora riconoscibili le tracce della struttura agricola delle tenute che fino alla fine dell'800 caratterizzavano

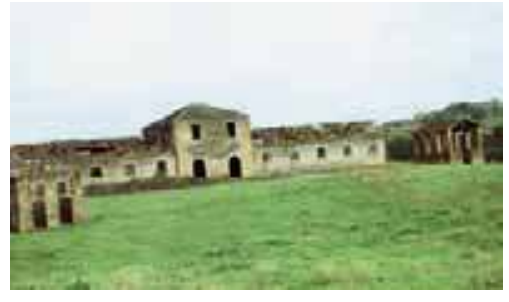

Figura 71. II casale Marco Simone Vecchio. quest'area e che sono ancora presenti con i resti di alcuni casali e vaccherie e del sistema delle fortificazioni, che aveva la funzione di controllo della viabilità che da Roma portava agli Appennini.

II paesaggio agrario in questa porzione di territorio non sembra avere connotazioni precise e peculiari, ma presenta una sorta di miscuglio tra gli elementi tipici di un paesaggio urbano e quelli agricoli, con piccoli orti, campi coltivati, edifici ed infrastrutture che si compenetrano e che conferiscono al paesaggio una visione complessiva di abbandono, degrado e confusione.

II rapporto tra paesaggio agrario ed edificato è sostanzialmente un rapporto di non riconoscibilità: l'edificazione non stabilisce alcun tipo di legame né con il supporto fisico, né con il contesto agricolo circostante che, al contrario, viene inglobato nel tessuto edificato perdendo ogni specificità. 
II ruolo dell'agricoltura, in questo contesto, non può dunque essere riferito ad un aumento in termini quantitativi delle superfici e delle produzioni, ma deve diventare il punto di riferimento sia per un miglioramento qualitativo dell'ambiente circostante e interno al costruito (produzioni agricole di qualità e spazi fruibili a servizio della città), sia i processi di valorizzazione delle identità specifiche dei luoghi (recupero a fini turistici di manufatti e complessi rurali).

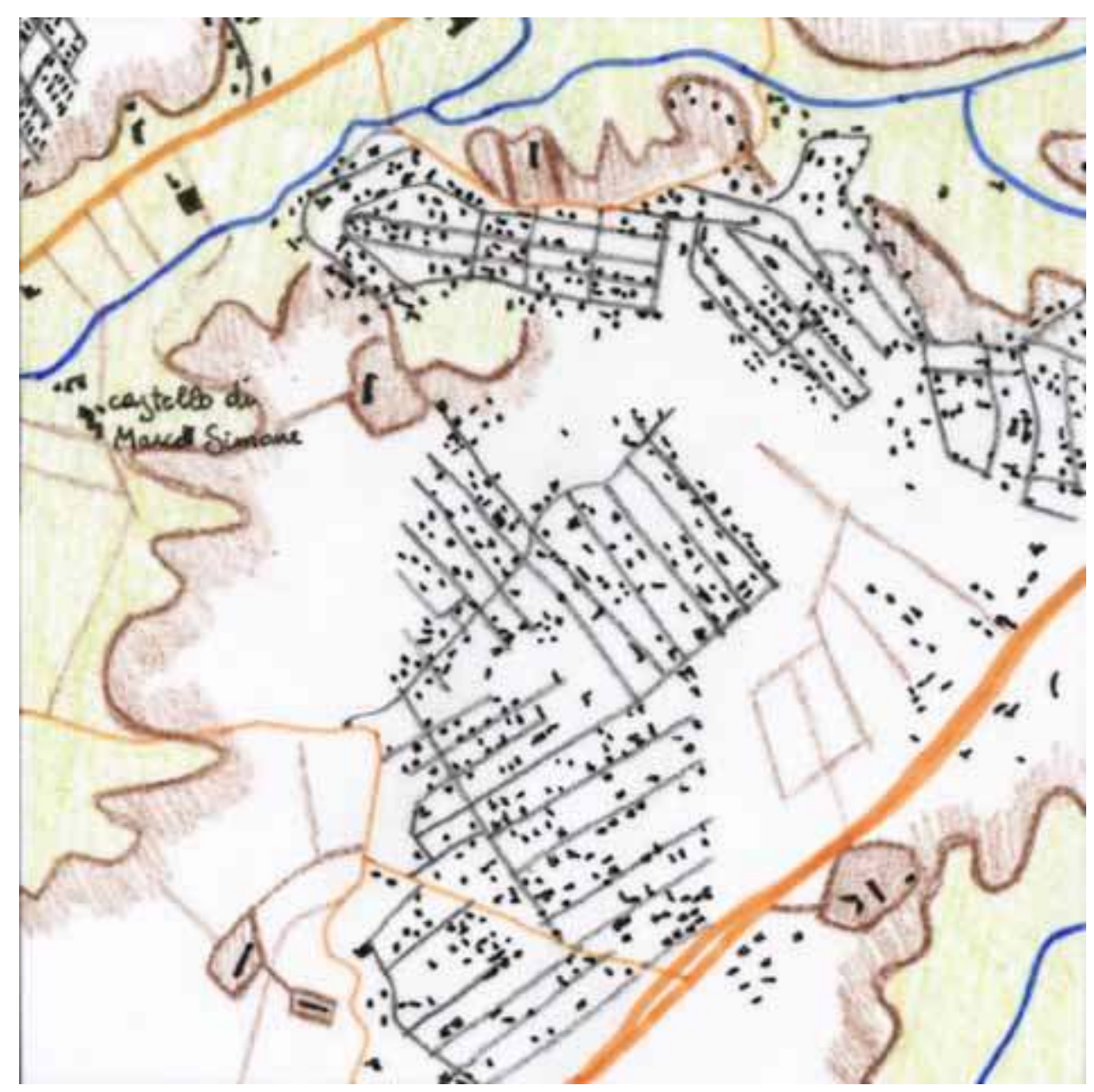

Figura 72. II rapporto tra edificato e paesaggio agrario non è riconoscibile e ciascun elemento si compenetra nell'altro in maniera del tutto indifferente ed autonoma. 


\section{SETTORE SUD-EST: CASAL MORENA}

Casal Morena è un'area situata a sud-est di Roma, nella quale convivono una realtà tipicamente urbana, talvolta densamente edificata, ed una realtà che presenta ancora significative caratteristiche agricole.

La rete infrastrutturale di livello territoriale presente - l'autostrada Roma-Napoli, le radiali Tuscolana e Anagnina, la ferrovia Roma-Frascati - ha rappresentato nello stesso tempo un attraversamento veloce del territorio, in quanto serviva a collegare Roma con i Castelli Romani, e un supporto per la maglia viaria secondaria.

La zona, dal punto di vista morfologico, rientra nelle ultime propaggini delle pendici del vulcano dei Colli Albani e presenta una morfologia articolata. L'intera regione a ridosso delle pendici vulcaniche è fortemente modellata dallo scorrere delle acque superficiali, che hanno inciso il territorio con valli strette, dovute all'origine soprattutto litoide di rocce molto resistenti all'erosione. I fondovalle, al contrario, sono ampi e piatti e separati da pianori aventi più o meno le stesse caratteristiche.

Dalla fine degli anni Settanta in poi, questa porzione di territorio è stata interessata da un processo insediativo particolarmente pesante e sono cresciute soprattutto le frange periferiche degli insediamenti consolidati - appartenenti sia a Roma, sia ai centri posti alle pendici dei Colli Albani come Ciampino - fino a formare un continuum intercomunale, interrotto ancora oggi solo da alcune discontinuità del territorio agricolo. Vengono impegnate con un'urbanizzazione diffusa, dalla forma scarsamente definibile e perimetrabile, le parti di "territorio intermedio"13 tra Roma e i Castelli, dove oggi ritroviamo le situazioni più disparate dal punto di vista insediativo.

13 Cfr. C. Nucci, A. Galassi, /l Parco regionale dei Castelli Romani, in B. Cignini, G. MASSARI, S. PIGNATTI (a cura di) «L'ecosistema Roma: ambiente e territorio», F.lli Palombi, Roma 1995, p.288.

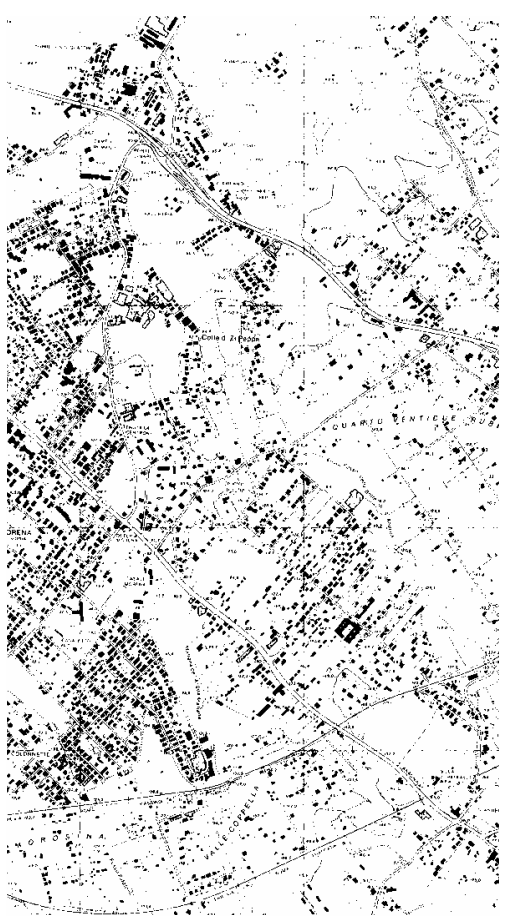

Figura 73. L'area di Casal Morena nel 1991. 


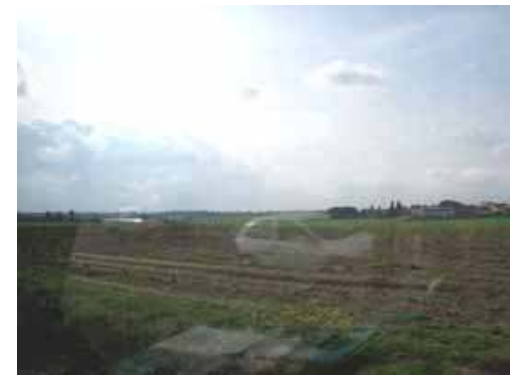

Figura 74. La tenuta ed il casale di Gregna - S. Andrea.

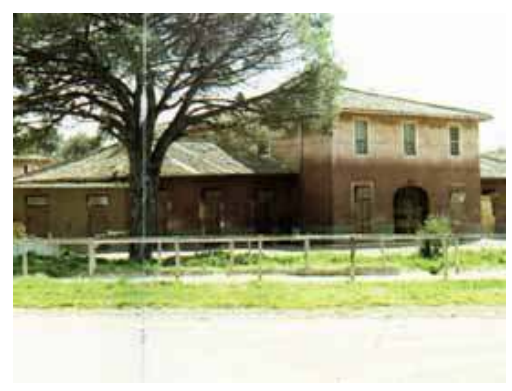

Figura 75. Un casale trasformato in struttura di servizio ad un maneggio.
Le periferie urbane vere e proprie (ne sono un esempio Giardini di Tor Mezzania, Osteria del Curato, Romanina vecchia, ecc...) sono addensate soprattutto nelle aree privilegiate localizzate lungo la rete infrastrutturale primaria e presentano un tessuto densamente edificato, nel quale le aree ancora libere sono poche e l'attività agricola è ormai scomparsa.

Gli insediamenti a bassa densità (ad esempio Morena e Vermicino), invece, si sono strutturati su una maglia viaria secondaria che ha seguito la regola dei frazionamenti delle antiche tenute.

Sono insediamenti caratterizzati dalla presenza di case con un massimo di tre piani, poste all'interno di lotti con giardino o che si alternano a piccoli appezzamenti agricoli.

Vi sono, infine, case sparse di tipo turistico - localizzate soprattutto nelle zone più prossime alle pendici dei Colli Albani - e insediamenti rurali di supporto alle attività agricole residue.

L'attività agricola si presenta anch'essa con conformazioni diverse.

Laddove, infatti, i processi di urbanizzazione delle lottizzazioni non hanno avuto luogo, vi sono ancora importanti porzioni di paesaggi agrari delle tenute, caratterizzati dalla presenza dei casali e delle strutture agricole annesse, dai filari alberati, dalle colture di tipo estensivo in appezzamenti di dimensioni medio-grandi.

Laddove, invece, sono avvenuti i frazionamenti delle lottizzazioni, l'agricoltura ancora coesiste tra le maglie del tessuto insediativo, sotto forma di orti urbani.

Dove, invece, sono presenti le strutture per le funzioni per il tempo libero metropolitano, ad esempio l'ippodromo delle Capannelle, il paesaggio agricolo ha perso completamente la sua funzione produttiva e sono scomparse le colture e l'attività agricola vera e propria. I manufatti agricoli, però, sono stati trasformati senza subìre alcuna modifica nel loro aspetto esteriore. 
Infine, è presente il paesaggio agricolo collinare delle prime pendici dei Castelli Romani che mantiene in gran parte la sua fisionomia originaria, caratterizzata dal disegno irregolare di piccoli appezzamenti disposti a formare una sorta di mosaico, dalle strutture insediative rurali dei casali e fabbricati minori, dalle ricche produzioni dei vigneti e uliveti.

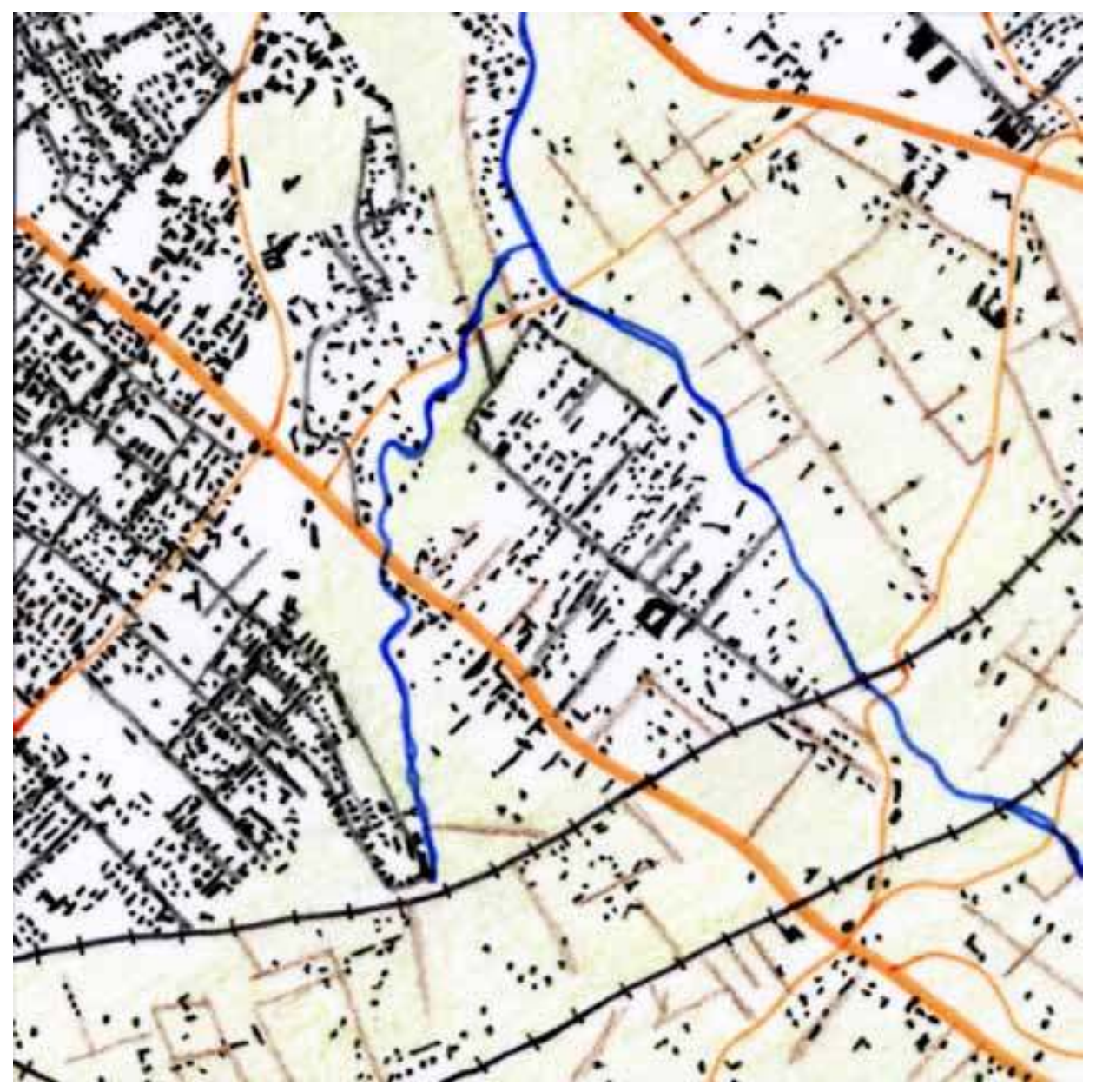

Figura 76. II rapporto tra edificato e paesaggio agrario è di mutua indifferenza: non si riconosce alcun tipo di condiziona-mento tra la struttura insediativa e quella paesistica.

II rapporto esistente tra paesaggio agrario e tessuto insediativo si potrebbe definire di indifferenza, in quanto l'attività agricola mantiene in alcune porzioni del territorio i caratteri e le funzioni originarie e convive accanto a situazioni urbane che con essa non hanno nulla a che vedere. II rapporto tra le parti che ne risulta è del tutto casuale ed è definito da 
un punto di vista meramente visivo, per l'accostamento tra parti costruite e parti non costruite. In questo caso non è possibile riscontrare, al di là dei rapporti visuali, alcun tipo di condizionamento tra la struttura insediativa e la struttura paesistica. 


\section{VALLECOLE ("VALLI STRETTE)}

dal carderistico profilo a $\vee$ particolarmente aspro.

TERRAZZI FLUVIALL

\section{VALLE ALLUVIONALE ("VALLE LARGA")
valle del Tevere.}

VALLI INCISE ("VALLI MEDIE"),
dal sistema idrografifo secondario, che si struttura
a pettine rispotto al corso d"'aciula principale

RIPIANI TUFACEI

rilievi collinari di modesta altezza, 50/60 mt, con
assenza di cime.

TERRAZZI FLUVIALL.

\section{VALLE ALLUVIONALE ("VALLE LARGA")}

valle dell'Aniene.

MEDIA PIANURA POCO INCISA
(zona nord) dolci ondulazioni su sediment

FORRE TUFACEE
(zona sud).

VALLECOLE ("VALLI STRETTE")
dal caratteristico profilo a V particolarmente aspro.

VALLI INCISE ("VALLI MEDIE")

dalla forma dolce, con pendenze prive di brusche

dalla torma
variazioni.

RIIIANI TUFACEI
rilisvi collinari di modesta altezza, 50/60 mt, con
assenza dicime

rilievi collinari di mod
assenza di cime.

in appezzamenti di ampie dimensioni, localizzati
unngo la valle dell'Aniene.
SEMINATIVI
in appezzamenti di ampie dimensioni, localizzati
nei fondovalle. PRATIE PASCOLI sui ripiani tufacei.

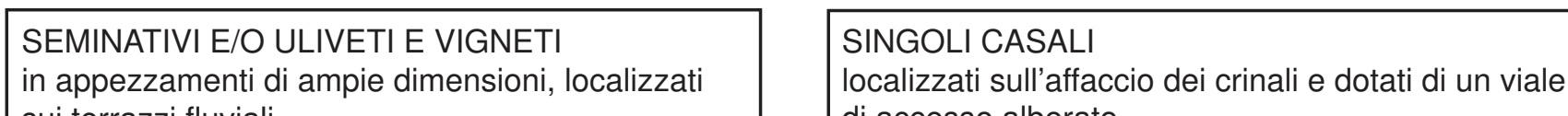

SEMINATIVI

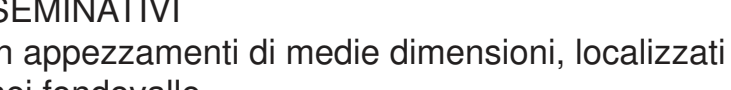

net tondovalle.
PRATIE PASCOL

sui ripiani turat
ULIVETI

sulle pendici dei rilievi della zona nord.

COLTURE IRRIGUE

in appezzamentid di ampie dimensioni, orditi
radialmente al fiume.

\section{COLTIVAZIONI ORTICOLE MISTE}

SEMINATIVI ESTENSIVI

nella zona nord. ULIVETI E ALTRI USI AGRICOLI SPECIALIZZATI in appezzamenti di piccole dimensioni, strutturati a
pettine rispetto alle forre nella zona sud.

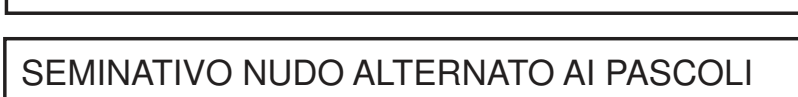

in appezzamentidi ampie dimensioni, localizzal

SEMINATIVO NUDO E/O FRUTTETI E ULIVETI
in appezzamenti di ampie dimensioni, localizzati in appezzamenti
sui ripiani tutacei.
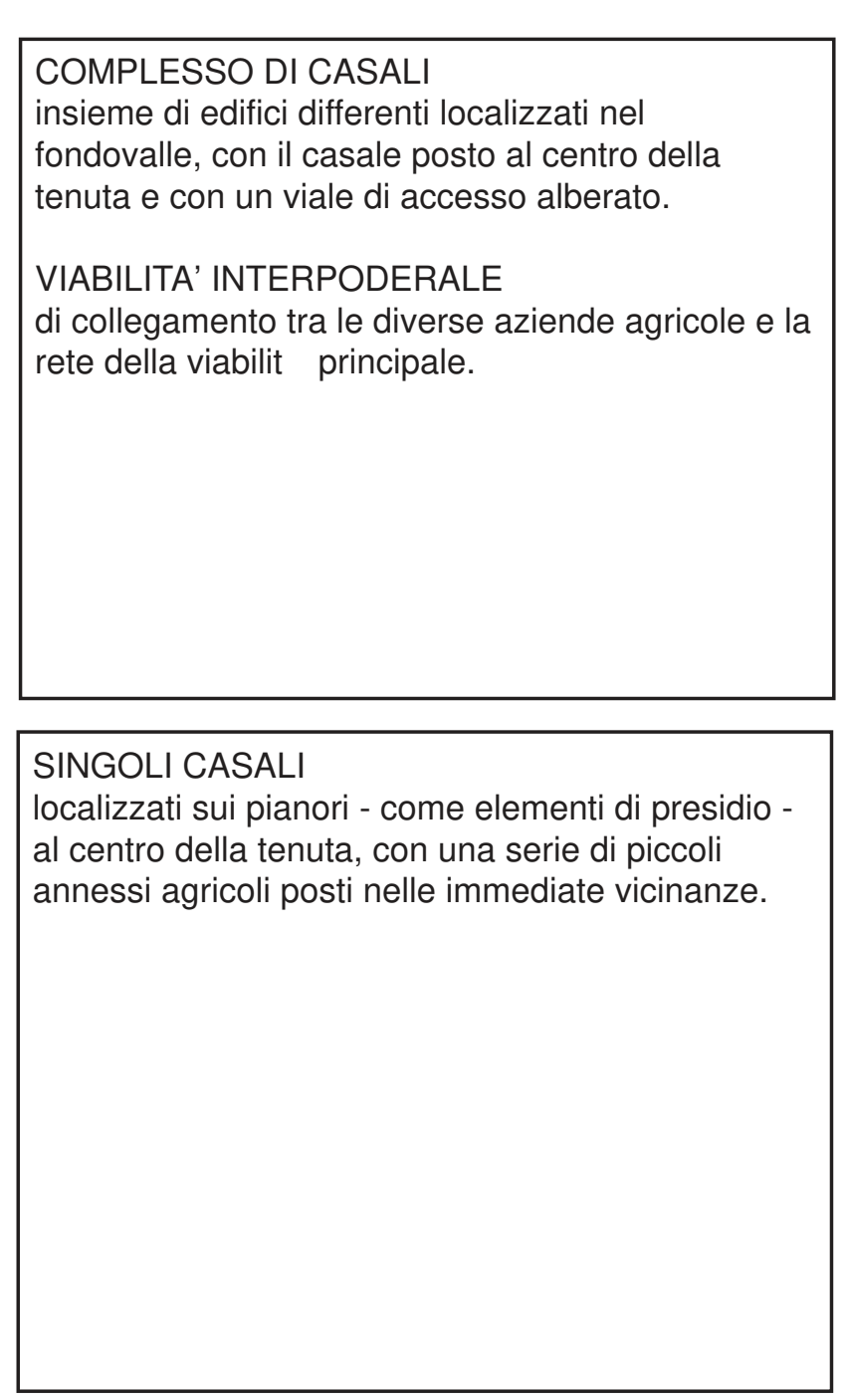

COMPLESSI FORESTALI

ben strutturati lungo i versanti e sulle sommit dei
vilievi collinari.

Alla confluenza del fosso della Magliana con il Tevere.

\section{di accesso alberato.}

COMPLESSO DI CASALI

Wreme di edifici differenti localizzati sulle

propaggini pie alte dei rilievi spartiacque, lungo la
viabilit di collegamento tra i punti sommitali.

CENTRI AGRICOLI

formati da complessi edilizi localizzati nelle ampie
vallate e sui pianori dellaz zona sud, con il casai vallate e sui pianori della zona sud, , con il casal posto al centro della ten
colture arboree di pregio

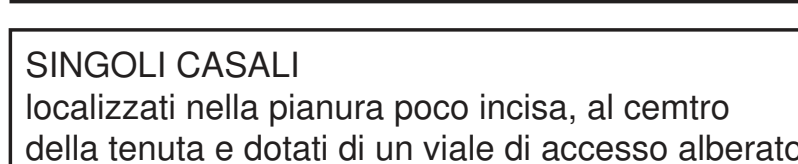

localizzati nella pianura poco incisa, al cemtro
della tenuta e dotati di un viale di accesso alberato diretto sulla viabilit principa

CASALI FORTIFICATI

localizzati - come elementi ilifensivi - sulle
propaggini pio alte presenti lungo la valle

COMPLESSO DI CASALI

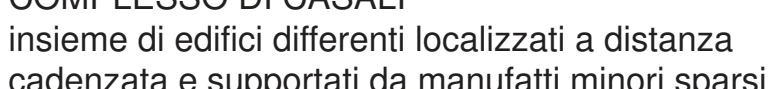
nella tenuta con il casale posto al minori spar nella tenuta con il casale posto al centro
tenuta e con un viale di accesso alberato.

VIABILITA' INTERPODERALE VIABLLITA' INTERPODERALE
di collegamento tra il casale e gli annessi agricoli
e/o la viabilit principale.

\begin{tabular}{|l|}
\hline COMPLESSI FORESTALI \\
ben strututuati i ungo i versanti e sulle sommit dei \\
rilievic colliniri-
\end{tabular} ilievi collinari.

PERCORSI STORICI - NECROPOLI - VILLE ad indicare come la zona rappresentasse una
zona urbana tanto in epoca pre-romana, quanto in epoca medievale.

\section{VEGETAZZONE RIPARIALE
lungo i corsi d'acacoue sulle scarpate piø acclivi.}

ACQUEDOTTI - VILLE

ad indicare come la zona costituisse
'approvigionamento idrico della citt e

rappresentasse
famiglie nobili.
AMBITI DI TERRITORI AGRICOLI SITUAZIONI URBASI COMPLETAMENTE DA L'ATTIVITA' AGRICOLA E GLI ELEMENTI PAESISTICI RISULTANO ANCORA

\begin{tabular}{|l|}
\hline CONCENTRAZIONI BOSCHIVE \\
di spalletta. \\
ZONE UMIDE \\
lungo le principali linee di compluvio. \\
PERCORSI STORIII \\
ad indicare come I zona costituisse la porta di \\
ingresso a Roma da nord. \\
\\
\hline
\end{tabular}

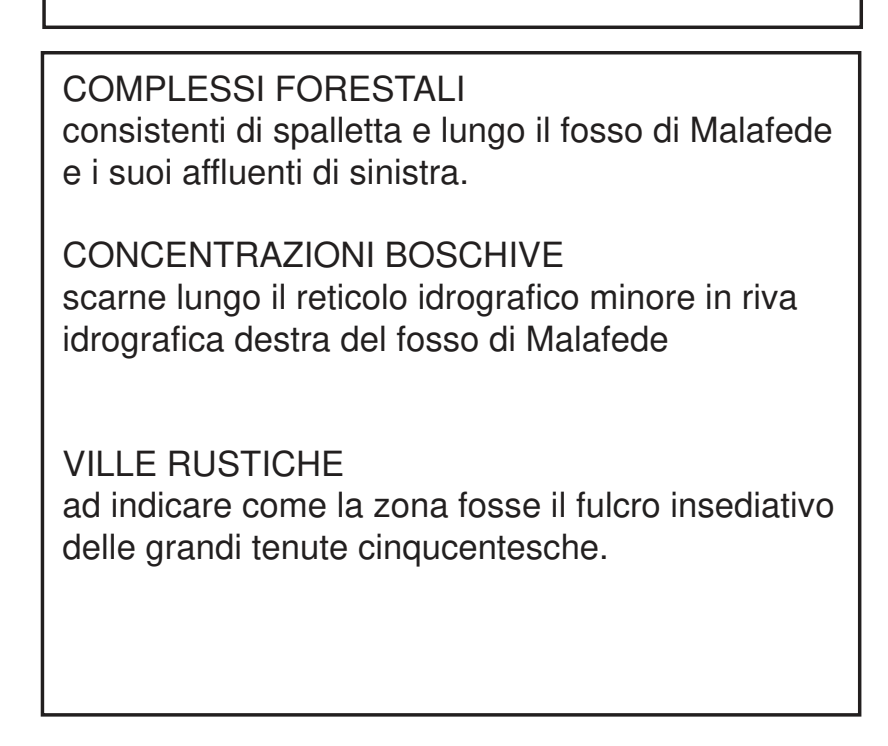
IRRISOLTE DELL'URBANIZZATO E PRESENTI
MEMORIA DELL'IDENTITA' STORICA D ELEMENTO INDISSOLUBILE NEL QUALE RINTRACEA

PORZIONI CONSISTENTI DI CAMPAGNE DELIMITATE DA IMPORTANTI ELEMENTI
MORFOLOGICIO INSEDIATIVI E TOCCATE SOLO MARGINALMENTE DA EPISOD URBANI, NELLE QUALI IL PAESAGGIO AGRARIO RAPPRESENTA ANC le forme ta suolo e delle sue sistemazioni de riconoscibilit dei rapporti tra insediamenti, limmaing spaziconivalle spazi boschivi, servizio della produzione agricola.

RETICOLO CONNETTIVO

necessario per mantenere rete ecologica continuit tra le diverse parti di ecosis naturall.

DISEGNO DEL MARGINE URBANO

RESIDUI DI CAMPAGNE INTERSTIZIALI, SOPRATTUTTO NEI SETTORI PERIFERICI
DELLA CITTA' IN PROSSIMITA' DELLE DELLA GTA, IN STOSSIMITA DEL

- mortologico e visivo - impedendo che le zone insediative si espandano a macchia d'olio e
senza regole nel territorio.

GRANDI INFRASTRUTTURE MODERNE.

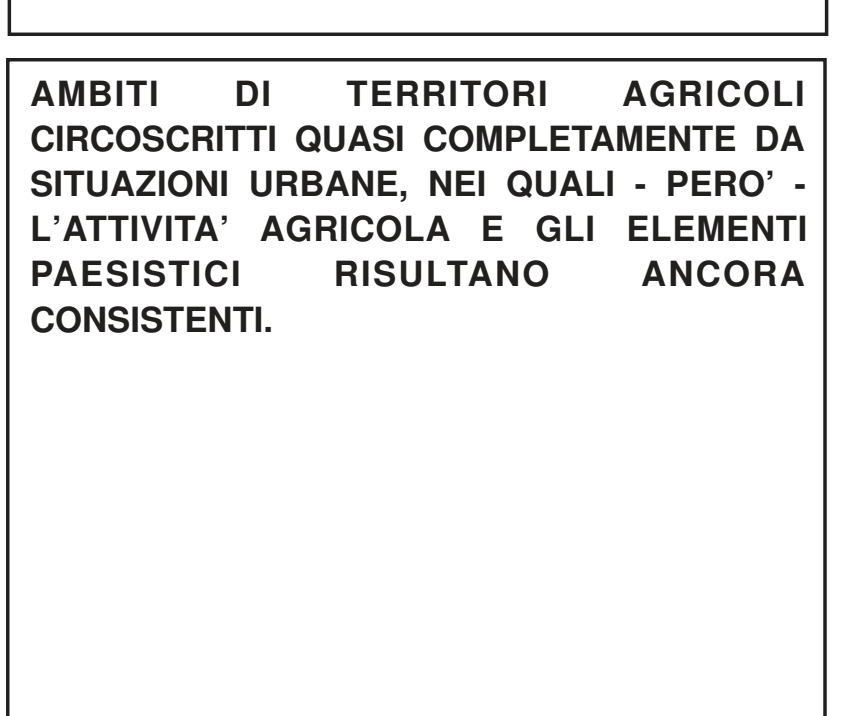


morfologia - idrografia

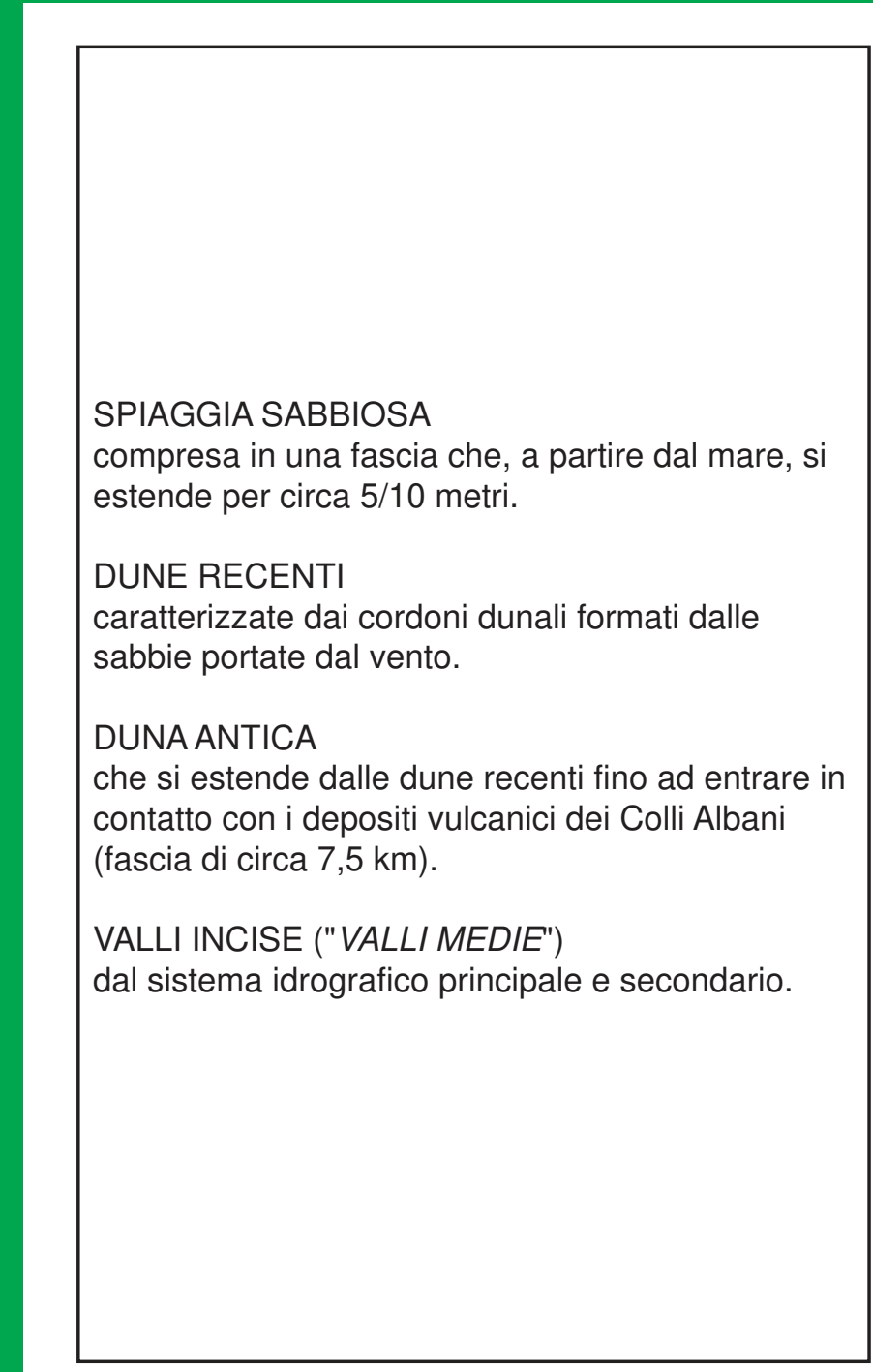

sistemazioni agrarie

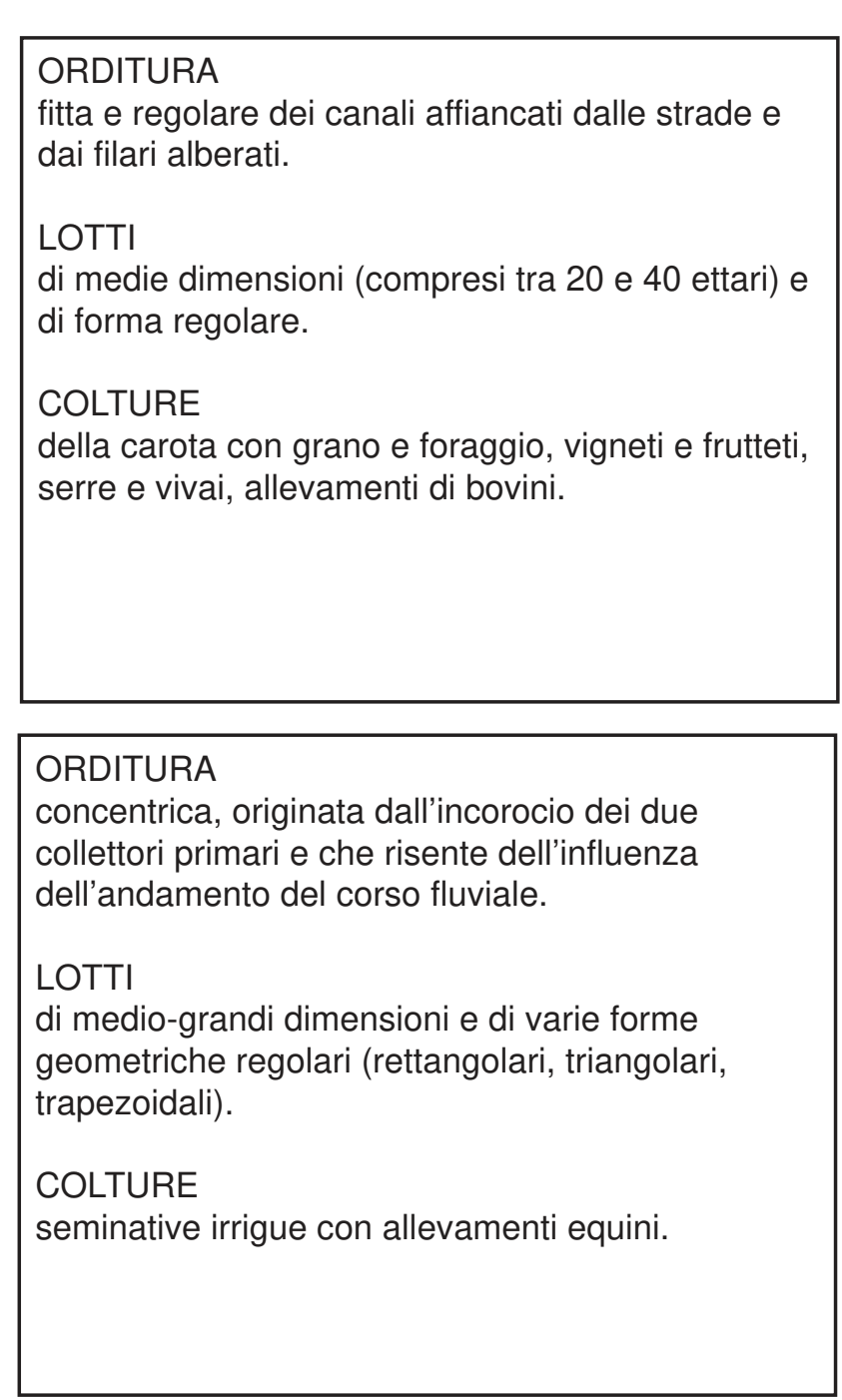

insediamento rurale

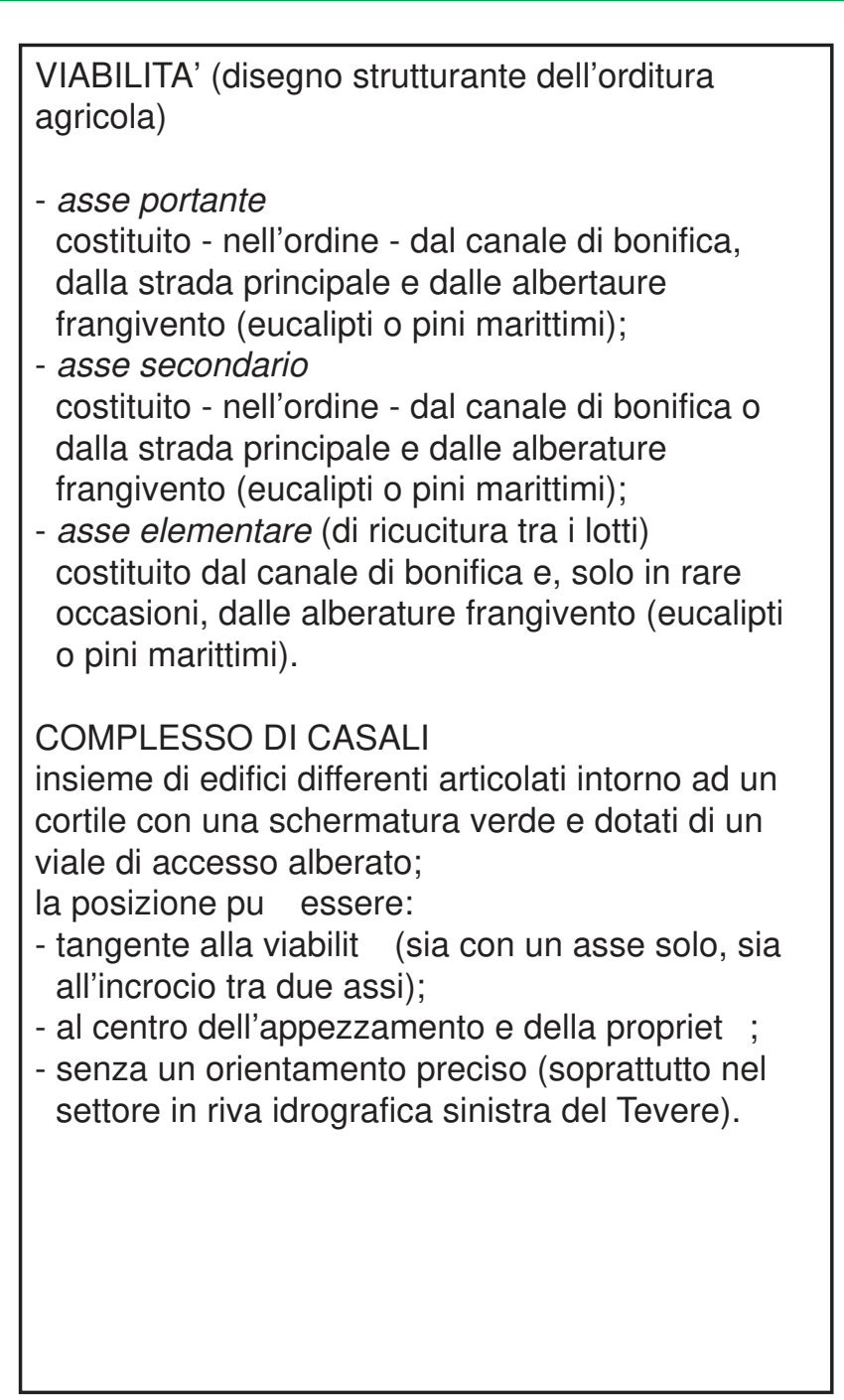

"residui" ambientali e storici

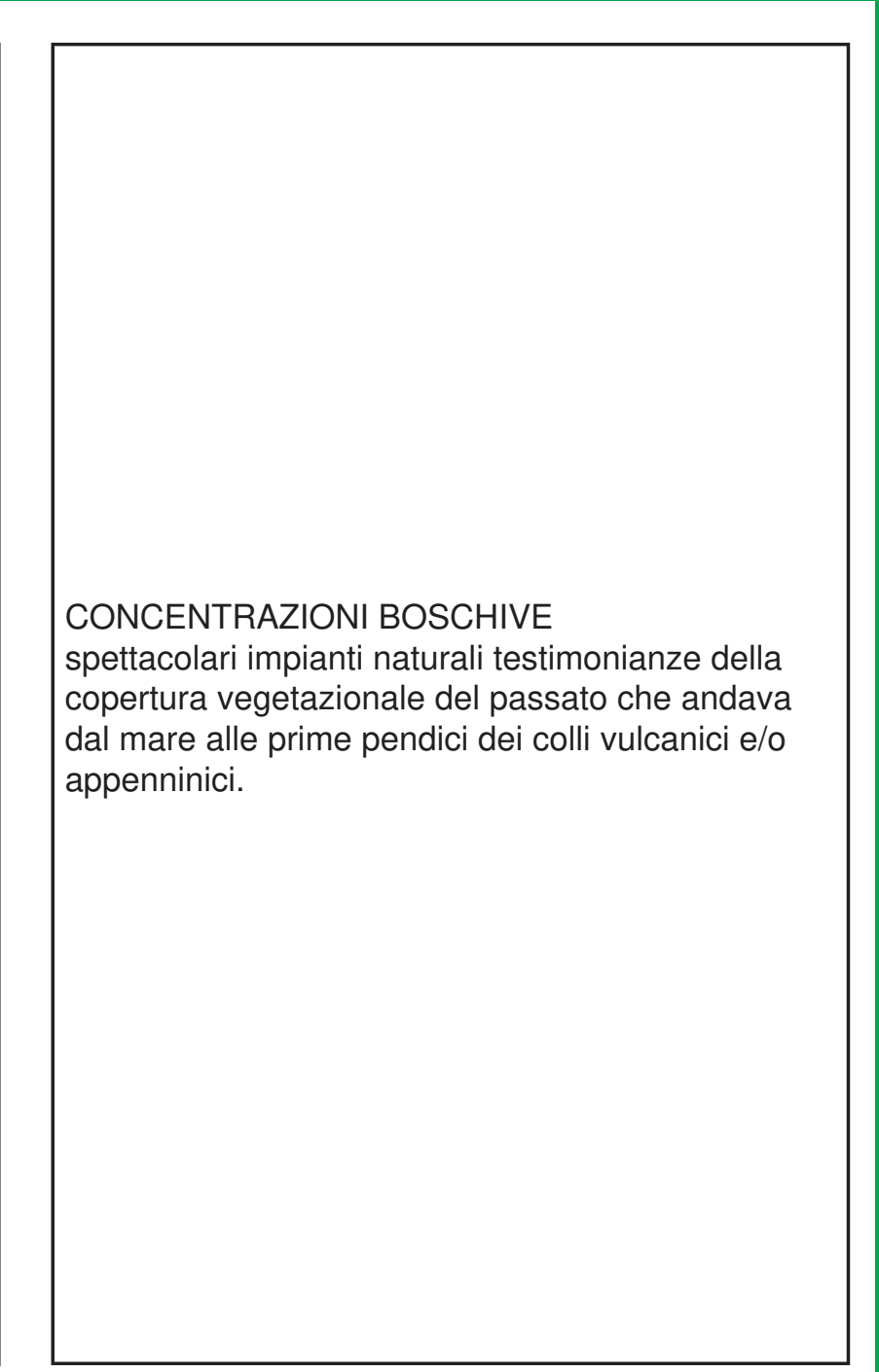

RAPPORTO URBANIZZATO /

PAESAGGIO AGRARIO

RUOLO DEL

RUOLO DEL
PAESAGGIO AGRARIO

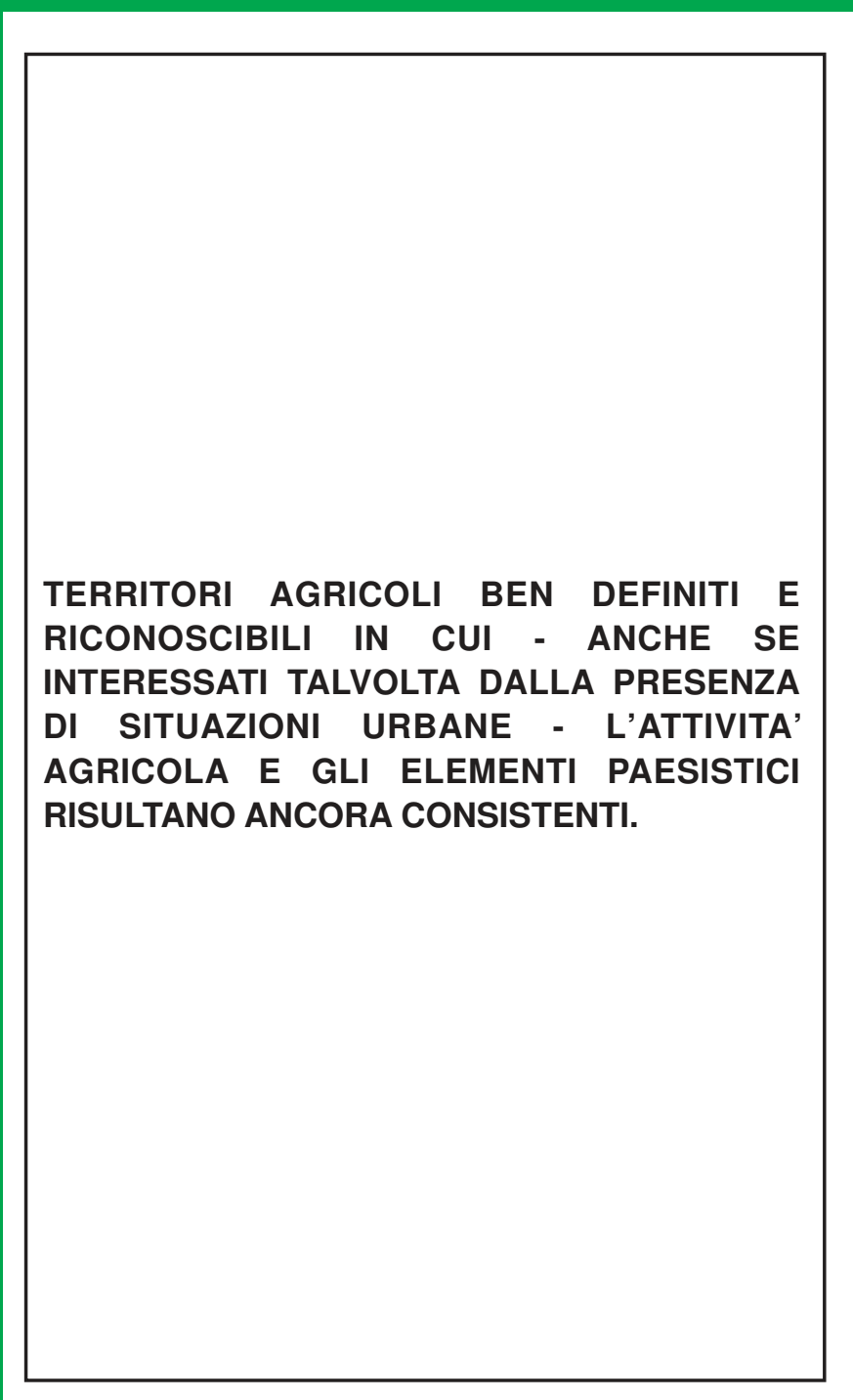

MEMORIA DELL'IDENTITA' STORICA DEI

ELEMENTO INDISSOLUBILE NEL QUALE
RINTRACCIARE LE REGOLE E LE MATRICI RINTRACCIARE LE REGOLE E LE MATRICI
STORICHE

suolo e delle sui impianti, la cura del riconoscibilit dei rapporti tra insediament, limmagiine di efficienza delle inti boschivi, servizio della produzione agricola.

RETICOLO CONNETTIVO

della rete ecologica contrative liverse parti di ecosistem

DISEGNO DEL MARGINE URBANO

- morfologico e visivo - impedendo che le zone

-

senza regole nel territorio. 
morfologia - idrografia

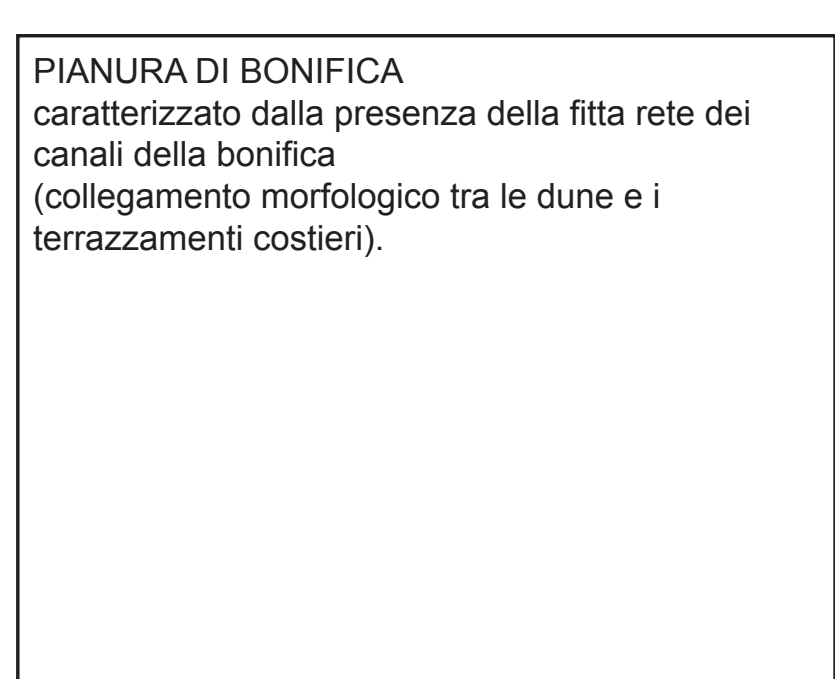

VALL INCISE ("VALLI MEDIE")

FORRE TUFACEE

FORRE TUFACEE
rilievi collinari i imodesta altezza, $50 / 60 \mathrm{mt}$, con
assenza di cime.

VALLI INCISE ("VALLI MEDIE")
dal sistema idrografico secondario dell'Aniene.

AMPI ALTOPIANI INCISI
dolci ondulazioni su sedimenti alluvional.

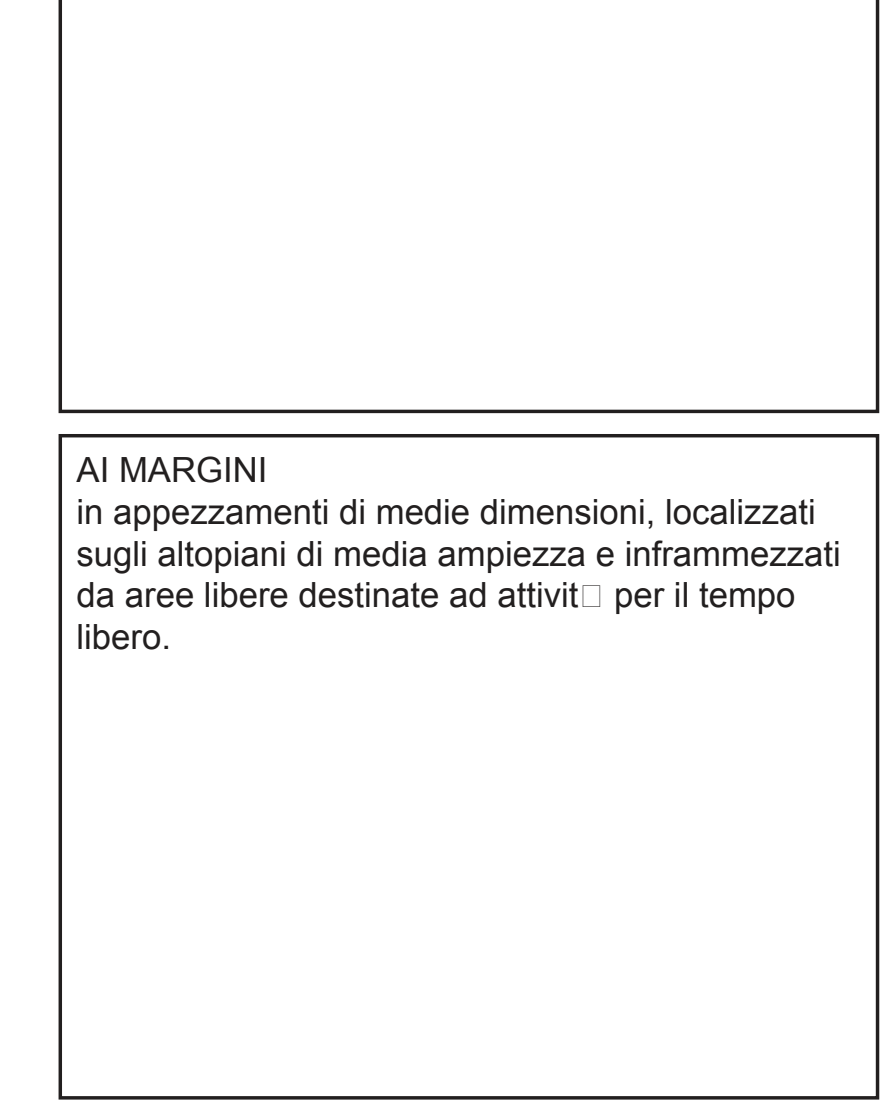
in appezzamenti di medie dimensioni, localizzati
suguli altopiani di media ampiezza e inframmezza da aree libere destinate ad attivit $\square$ per il tempo

dibero.

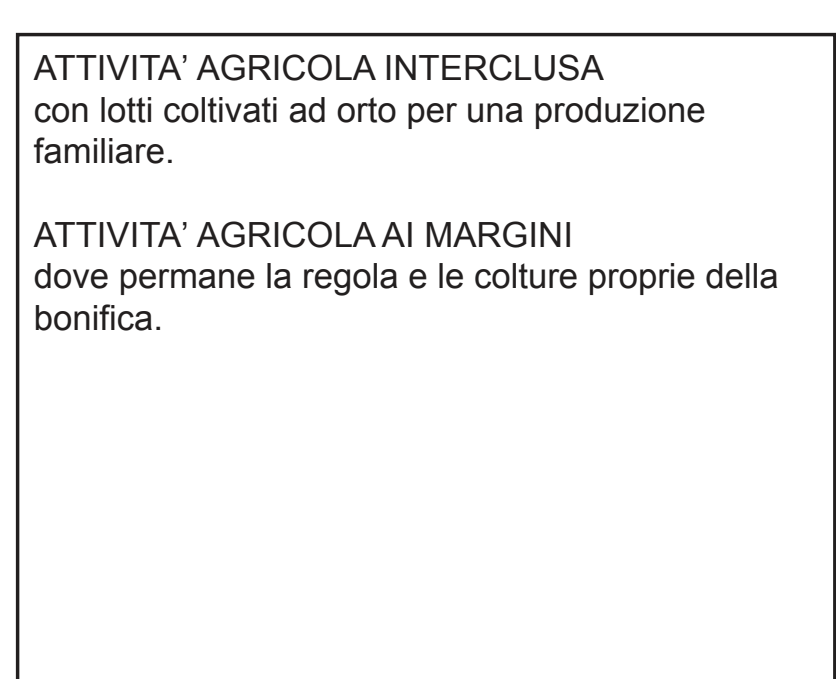

in appezzamenti di medie dimensioni, localizzati
sui pianorie - talvolta - nei fondovalle di media
ampiezza.

VALLI INCISE ("VALLI MEDIE)

dalla forma dolce, con pendenze prive di brusche

RIPIANI TUFACEI

rilievi collinari di modesta altezza, 50/60 $\mathrm{mt}$ con

assenza di cime.

PORZIONI DI PAESAGGIO AGRARIO DELLE dove i processsi di urbanizzzazione delle Iotitizzazioni
non hanno avuto luogo e dove $k$ ancora possibile rintracciare:

CENTRI AGRICOLL, formati da complessi edilizi
Iocalizzati sui ripiani tufacei, con il casale posto al \begin{tabular}{|l|} 
AI MARGINI \\
in appezzamenti di medie dimensioni, Iocalizzati \\
sui pinzori e- talyolta
\end{tabular} insediamento rurale

insediamento urbano
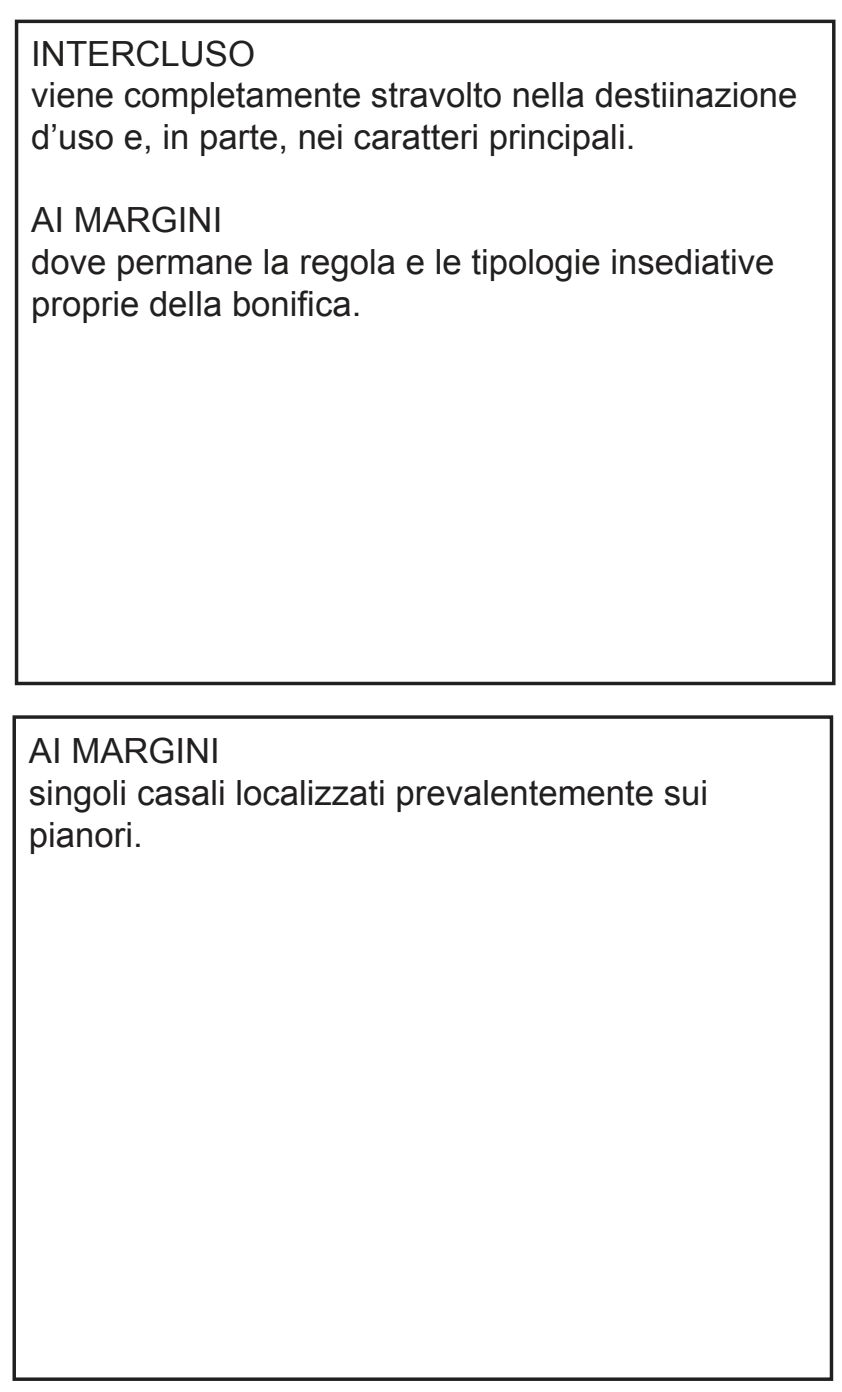

PAESAGGIO AGRICOLO PERIURBANO
con orti urbani destinati ad una produzione

con orti urbani destinati ad una produzione
familiare e cararterizzati dalla presenza di tipologie

edilizie urbane.

CENTRI AGRICOLI
formati da complessi edilizi localizzati sug

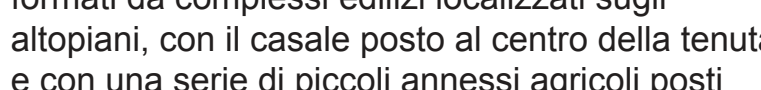
e con una serie di piccol
nelle immediate vicinanze.

CASALI FORTIFICATI propaggini piø alte presentil lungo la valle$$
\text { PAESAGGIO AGRICOLO PERIURBANO }
$$
localizzati suir ripiani itufacel, con II casale posto
centro dell tenuta e con una serie di piccoli
annessi agricoli posti nelle immediate vicinanze;

SEMINATIVI ESTENSIVI, in appezzamenti medio-
PAESAGGIO AGRICOLO PERIURBANO con orti urbani destinati iad una produzione
familiare e caratterizzati ialla presenza di tipologie edilizie urbane.

RICONVERSIONE DEI MANUFATTI AGRICOLI dove sono nat tempo libero e dove sit contenitor" di attivitt perpletame funzione produttiva del passato. prevalenza di villini mono-plurifamiliari.

EDIFICAZIONE DIFFUSA
con larghi spazi liberi, carattizz

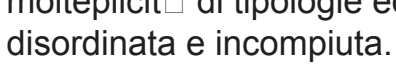

TESSUTO RETICOLARE

se non nella regolarit della maglia della

elementi propri della bonifica e caratterizzato dall

urban

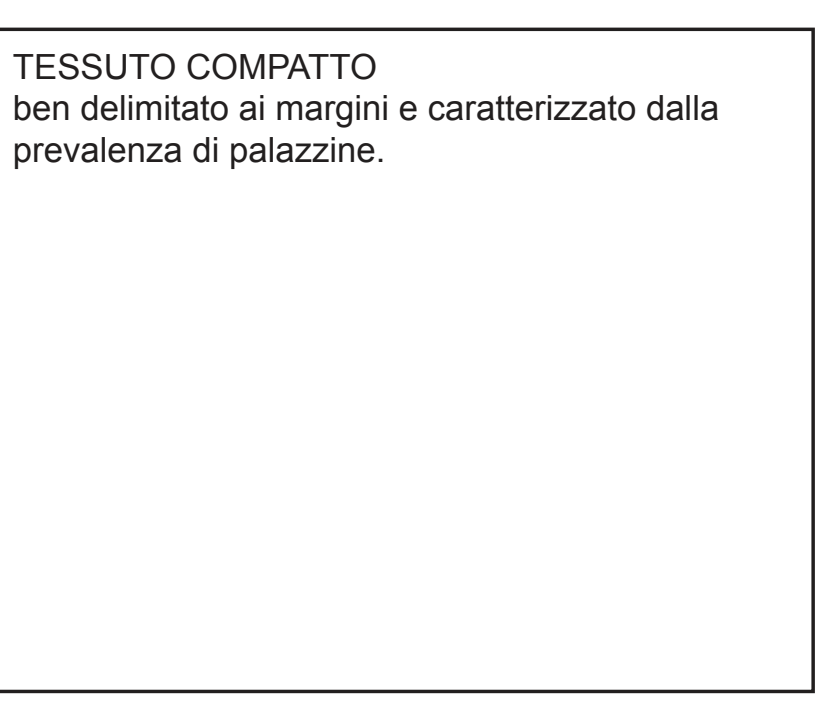

con larghi Spazi liberi, caratterizzata da una
molteplicitit di tipologie edilizize e da ua

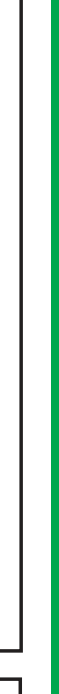

EDIFICAZIONE DIFFUSA
con larghi spazi liberi, caratterizzata da una
molteplicit di dipologie edilizie a bassa densit $t$ e derivante dai frazionamenti delle antiche tenute
(frange perfieriche degli insediamentic onsolidati
tanto di Roma, quanto dei Castelli Romani).

RAPPORTO DI INDIFRERMIA / NON RAPPORTO DIII
RICONOSCIBLE

dove non si riconosce alcun tipo condizionamento tra la struttura insediativa $\mathrm{e}$ quella paesistica.
L'attivit agricola

'attivit $\square$ agricola mantiene in alcune porzioni
di territorio i caratterie

di territorio i caratteri e le funzioni or
convive accanto a situazioni urbane.

RAPPORTO DI
RICONOSCIBILE

ciascun elemento si compenetra

autonoma.
L'edificato ingloba al suo interno il contesto grand.
ELEMENTO ORDINATORE

insediamenti, contribuiscono a dare una $\mathrm{gll}$

compiuta al tessuto urbano.

RETICOLO CONNETTIVO

della rete ecologica

diffusa.

DISEGNO DEL MARGINE URBANO

- morfologico e visivo - impedendo che la zona

MEMORIA DELL'ID
LUOGHI

caratterizzata dalla trama dalle colture
sstensive a rotazione tipiche dell'agro romano edalla strutura inseditiva dic ascar post

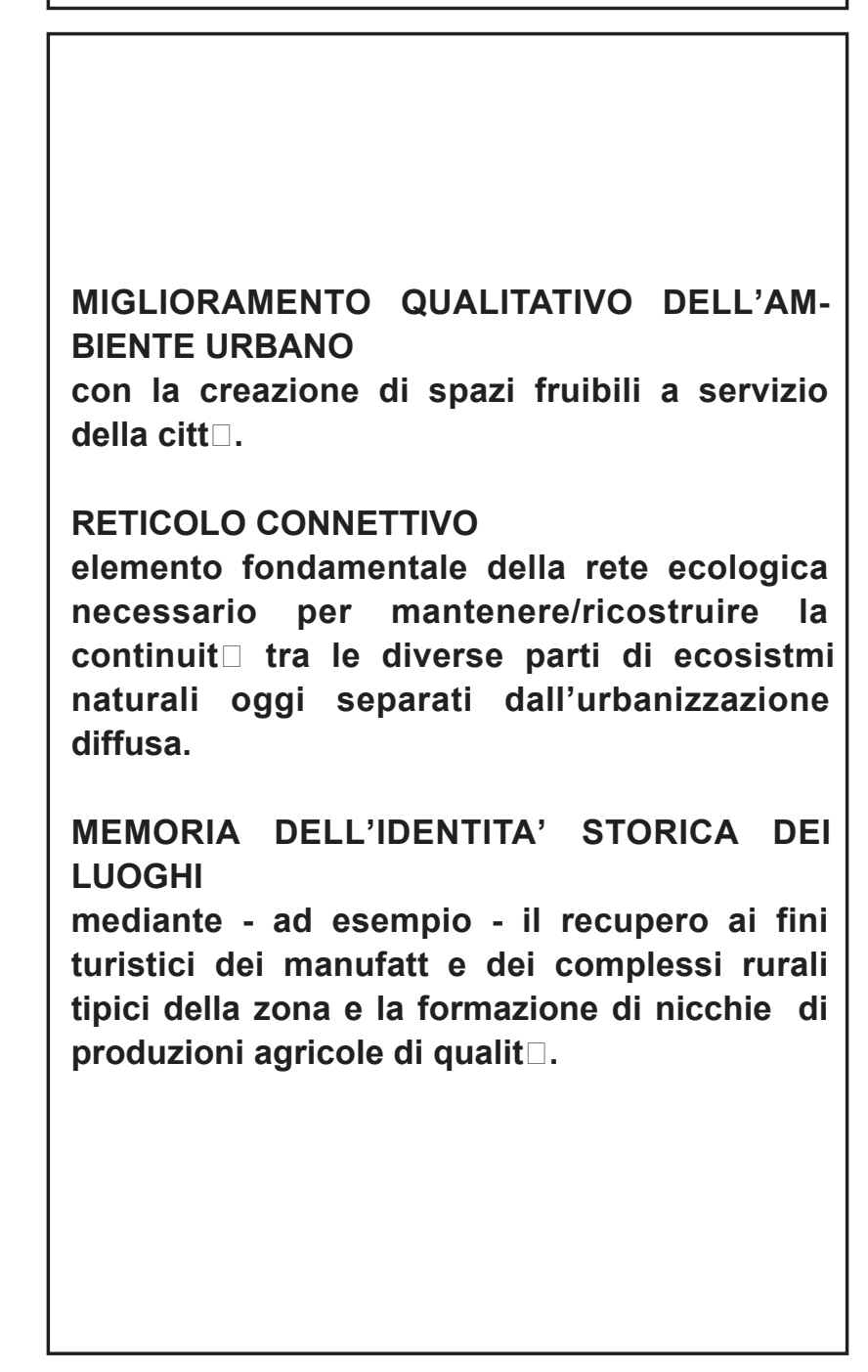




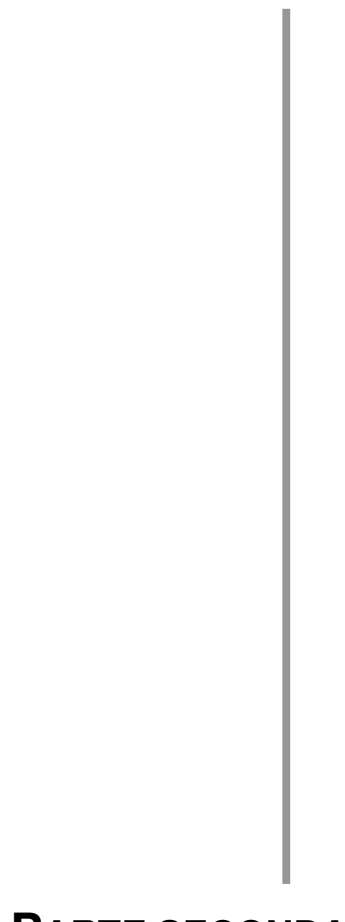

PARTE SECONDA

BIBLIOGRAFIA SPECIFICA DI RIFERIMENTO

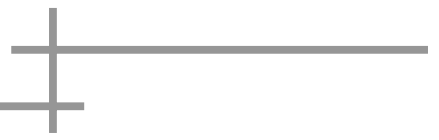


Farsi una bibliografia significa cercare quello di cui non si conosce ancora l'esistenza. ${ }^{1}$

\section{SULLA DELIMITAZIONE E DESCRIZIONE DEL CAMPO APPLICATIVO}

- AA.Vv., Ambienti di particolare interesse naturalistico del Lazio, Regione Lazio - Assessorato alla Cultura e Dip.to di Biologia Vegetale dell'Università di Roma "La Sapienza", Roma 1996.

- $\quad$ Almagià R., Lazio, UTET, Torino 1966.

- Amadio V., CApannelli E., I/ sistema ambientale, dalla marginalità alla valorizzazione, in AA.VV. «La città metropolitana di Roma. Un metodo per la delimitazione», Ires, Roma 1991, pp. 11-21.

- Arnoldus Huyrendeveld A. e altri, Il paesaggio e i geotopi della campagna romana, F.lli Palombi, Roma, s.d.

- Battisti C., Le connessioni tra aree naturali attorno alla città di Roma, in C. DI MAGgIO e R. GHIRINGHELLI (a cura di), «Reti ecologiche in aree urbanizzate», Quaderni del Piano per l'area metropolitana milanese n.13, Franco Angeli, Milano 1999, pp. 99102.

- $\quad$ Bortolotti L., Roma fuori le mura, Laterza, Roma - Bari 1988 .

- Bortolotti L., Le persistenze della campagna romana: alcuni aspetti della sua evoluzione storica, intervento al seminario "Strumenti e metodi per la conoscenza della campagna romana», Dottorato di ricerca in Progettazione Paesistica, Roma 2000.

- Brancati A., La cartografia dell'Agro Romano, F.lli Palombi, Roma 1990.

- CABIANCA V., QUILICI L., I beni culturali archeologici del territorio romano, in «Urbanistica» nn.54-55, set., INU edizioni, Roma 1969, pp. 82-108.

- Cafiero G. (a cura di), La pianificazione del sistema delle aree protette di Roma. Le nove Riserve naturali di RomaNatura, «Urbanistica Quaderni» n.37, mar., INU edizioni, Roma 2003.

- Calzolari V., Ambito e ambiti della campagna romana come letture e prospettive per la pianificazione, intervento al seminario

1 U. Eco, Come si fa una tesi di laurea. Le materie umanistiche, Bompiani, Milano 1977, p.66. 
«Strumenti e metodi per la conoscenza della campagna romana», Dottorato di ricerca in Progettazione Paesistica, Roma 2000.

- CAlzolari V., Beni culturali e valori storico-paesistici delle aree protette di RomaNatura, in «Urbanistica Quaderni» n.37, mar., INU edizioni, Roma 2003, pp. 32-34.

- CALZOLARI V., II sistema storico - ambientale dell'area romana, in B. CIGNINI, G. MASSARI, S. PIgNATTI (a cura di) «L'ecosistema Roma: ambiente e territorio», F.lli Palombi, Roma 1995, pp. 136-147.

- CAlzolaRI V., Le interrelazioni e la varietà, in V. CALzOLARI «Storia e natura come sistema: un progetto per il territorio libero dell'area romana», Àrgos, Roma 1999, pp. 101-134.

- Calzolari V., Storia e natura come sistema: un progetto per il territorio libero dell'area romana, Àrgos, Roma 1999.

- CARAcciolo A., QUILICI F., Roma, una capitale singolare, il Mulino, Bologna 1985.

- Caravaggi L., Risorse ambientali primarie e sistema, in V. CALZOLARI «Storia e natura come sistema: un progetto per il territorio libero dell'area romana», Àrgos, Roma 1999, pp. 199-202.

- CARoselli M.R., La realtà nella storia agricola romana, in «Rivista di storia dell'agricoltura» n.3, a.XI, 1971, pp. 266-279.

- Cazzola A., Campagna romana o campagne romane?, in «Bollettino del DUPT della Facoltà di Architettura di Firenze» n.1-2, Dipartimento di Urbanistica e Pianificazione del Territorio, Firenze 2001, pp. 70-71.

- Celesti Grapow L., Petrella P., Atlante della flora di Roma. La distribuzione delle piante spontanee come indicatore ambientale, Àrgos, Roma 1995.

- Cherubini L., Roma e dintorni, II Lupo \& Co., Roma 2001.

- Cignini B., Massari G., Pignatti S. (a cura di), L'ecosistema Roma: ambiente e territorio, F.lli Palombi, Roma 1995.

- Coarelli F. (a cura di), II Lazio. Guide Archeologiche, Laterza, Roma - Bari 1984.

- Cosentino D., Parotto M., Praturlon A., Guide geologiche regionali. II Lazio, Be-Ma, Milano 1993.

- Di Giovine M., Squitieri G., Piano di azione ambientale di Roma: documento preliminare, Comune di Roma, Roma 1997.

- Gisotti G., Zarlenga F., La geologia della città di Roma tra urbanistica e archeologia, in «Geologia dell'ambiente» serie VI, n.4, 1998, pp. 45-58. 
- Gross H., Roma nel Settecento, Laterza, Roma - Bari 1990.

- NibBy A.E., Analisi storico-topografica antiquaria della carta dei dintorni di Roma, Forni, Bologna 1973 (ristampa anastatica della versione originale del 1837).

- Paratore E., II suburbio geo-agrario di Roma, Istituto di Studi Romani, Roma 1979.

- RonZANi A., II ruolo dei parchi prenestini nel sistema metropolitano romano, in «Paesaggio Urbano» n.1, gen.-feb., Maggioli, Rimini 1996, pp. 84-95.

- TomassetTi G., La campagna romana antica, medievale, moderna, Arnaldo Fonti, Sala Bolognese 1976.

- Touring Club Italiano, Guida d'Italia: Lazio, Touring Club Italiano, Milano 1977.

- VAlenti V., La cartografia tematica del territorio: caratteristiche e potenzialità agricole della provincia di Roma, Officina, Roma 1984.

- Ventriglia U., Idrogeologia della provincia di Roma, (a cura della Provincia di Roma - Ass.to LL.PP. Viabilità e Trasporti), Roma 1990.

\section{SULLE CAUSE ED EFFETTI DELLE PRINCIPALI TRASFORMAZIONI NEL PAESAGGIO DELLA CAMPAGNA ROMANA}

\section{Sulla proprietà e la rendita}

- $\quad$ Bortolotti L., Roma fuori le mura, Laterza, Roma - Bari 1988.

- BortolotTI L., Le persistenze della campagna romana: alcuni aspetti della sua evoluzione storica, intervento al seminario «Strumenti e metodi per la conoscenza della campagna romana», Dottorato di ricerca in Progettazione Paesistica, Roma 2000.

- CAU A., VILLARINI M., Indagini sulla struttura della proprietà fondiaria, sui tipi d'imprese agricole e sulla utilizzazione del suolo nel comprensorio consortile e nelle zone limitrofe del Comune di Roma, Consorzio per la bonifica dell'Agro Romano, Roma 1972.

- De Felice R., Aspetti e momenti della vita economica di Roma e del Lazio nei secoli XVIII e XIX, Edizioni di Storia e Letteratura, Roma 1965. 
- De Felice R., Storia dell'Italia contemporanea, Edizioni Scientifiche Italiane, Napoli 1976.

- De Felice R., Castronovo V., Società e costume nell'Italia unita, Istituto Poligrafico e Zecca dello Stato, Roma 1994.

- De Sismondi S., Del metodo di restaurare l'agricoltura nella campagna romana, s.e., s.d.

- Della Seta P., Della Seta R., I suoli di Roma. Uso e abuso del territorio nei cento anni della capitale, Editori Riuniti, Roma 1988.

- Della Seta R., La liquidazione dell'asse ecclesiastico nell'Agro Romano, in «Storia urbana», n. 40, lug.-set., Roma 1987, pp. 99118.

- Gross H., Roma nel Settecento, Laterza, Roma - Bari 1990.

- Guerreri G., La liquidazione dell'asse ecclesiastico nella Campagna Romana. Vecchi e nuovi proprietari; cambiamenti e permanenze, in «Storia urbana», n. 42, gen.-mar., Roma 1988, pp. 85-144.

- MANCINI O., Il territorio agricolo dell'area metropolitana di Roma, in P. BOLCHINI, D. LORANDI (a cura di), «Metropoli e agricoltura», Franco Angeli, Milano 1982, pp. 255-278.

- Orlando G., Le campagne: agro e latifondo, montagna e palude, s.e., s.d., pp. 83-128.

- Paratore E., Il suburbio geo-agrario di Roma, Istituto di Studi Romani, Roma 1979.

- Prete M.R., Fondi M., La casa rurale nel Lazio settentrionale e nell'Agro romano, Olshki, Firenze 1957.

- Rossi G., L'agro di Roma tra '500 e '800: condizioni di vita e lavoro, Edizioni di storia e letteratura, Roma 1988.

- Russo S., Una dimora per leggere il paesaggio, in «Viaggi di Erodoto», II, n. 5, sett., Roma 1988, pp. 98-107.

- Sombart W., La campagna romana, Loescher, Torino 1891.

- TAgliaferRi C., I casali della campagna romana, Pieraldo, Roma 1991.

- Vallino F.O., Melella P., Tenute e paesaggio agrario nel suburbio romano sud-orientale dal sec.XIV agli albori del Novecento, in «Bollettino della Società Geografica Italiana» vol.XII, Roma 1983. 


\section{Sulle opere di bonifica}

- BeVilacqua P., Rossi Doria M. (a cura di), Le bonifiche in Italia dal '700 ad oggi, Laterza, Roma - Bari 1984.

- $\quad$ Bortolotti L., Roma fuori le mura, Laterza, Roma - Bari 1988.

- CAdolini G., II bonificamento dell'Agro romano, s.e., Roma 1901.

- Cacherano di Bicherasio G. F. M., II ripopolamento dell'agro romano, in P.BEVILACQUA e M. ROSSI DORIA (a cura di) «Le bonifiche in Italia dal '700 a oggi', Laterza, Roma - Bari 1984, pp. 132-137.

- De CuPIS C., Le vicende dell'agricoltura e della pastorizia nell'Agro Romano, Tipografia Nazionale di G. Bertero, Roma 1911.

- Celli A., Contadini nella Campagna Romana, in P. Bevilacqua e M. Rossı DORIA (a cura di) «Le bonifiche in Italia dal '700 a oggi», Laterza, Roma - Bari 1984, pp. 234-243.

- Lattanzi G., LATTANZI V., La bonifica del litorale di Roma, 18701911, in AYMONINO C. (a cura di), <<Architettura e urbanistica. Uso e trasformazione della città storica >>, Marsilio, Venezia 1984, pp. 142-151.

- Lattanzi G., Pane e lavoro: storia di una colonia cooperativa. I braccianti romagnoli e la bonifica di Ostia, Marsilio, Venezia 1986.

- Opera Nazionale per i Combattenti, 36 anni dell'Opera nazionale per i combattenti: 1919-1955, (a cura dell'Ente), Firenze 1955.

- Parisi Presicce A., VILletti G., Le bonifiche: un ponte fra passato e futuro, in C. BAGNASCO (a cura di), «ll delta del Tevere: un viaggio fra passato e futuro», F.lli Palombi, Roma 1998, pp. 97-109.

- $\quad$ SOMBART W., La campagna romana, Loescher, Torino 1891.

\section{Sull'infrastrutturazione del territorio}

- Ascarelli G., Ferrovie e tramvie nello sviluppo dei trasporti urbani ed interurbani, in AYMONINO C. (a cura di), «Architettura e urbanistica. Uso e trasformazione della città storica», Marsilio, Venezia 1984, pp. 129-141.

- $\quad$ Bortolotti L., Roma fuori le mura, Laterza, Roma - Bari 1988.

- Bortolotti L., Le persistenze della campagna romana: alcuni aspetti della sua evoluzione storica, intervento al seminario "Strumenti e metodi per la conoscenza della campagna romana», Dottorato di ricerca in Progettazione Paesistica, Roma 2000. 
- $\quad$ Cuccia G., Urbanistica Edilizia Infrastrutture di Roma Capitale 1870 - 1990, Laterza, Roma - Bari 1991.

- Ghisellini V. (a cura di), L'Autostrada del Grande Raccordo Anulare, USPR - Documenti 10 (pubblicazione a cura del Comune di Roma), Roma s.d.

- FrIZ G., Le strade dello Stato Pontificio nel XIX sec., Archivio economico dell'unificazione italiana, Roma 1967.

\section{Sull'urbanizzazione e la città metropolitana}

- AA.Vv., Roma città e piani, «Urbanistica» nn. monografici 27 e 2829, INU edizioni, Roma 1959.

- $\quad$ AA.Vv., Roma, il piano delle periferie: materiali per un dibattito, L'ed edizioni, Roma 1993.

- AA.Vv., Gli sviluppi del piano a Roma, in «Urbanistica» n.30, mar., INU edizioni, Roma 1960, pp. 97-118.

- Bartolini F., Roma borghese. La casa e i ceti medi tra le due guerre, Laterza, Roma - Bari 2001.

- Benevolo L., Roma da ieri a domani, Laterza, Roma - Bari 1971.

- Benevolo L., Città in discussione: Venezia e Roma, Laterza, Roma - Bari 1979.

- Benevolo L., Roma dal 1870 al 1990, Laterza, Roma - Bari 1992.

- Berdini P. (a cura di), La città senza piano: le trasformazioni urbanistiche di Roma negli anni '80, Istituto Nazionale di Urbanistica - sezione Lazio, Roma 1992.

- Bortolotti L., Roma fuori le mura, Laterza, Roma - Bari 1988.

- Bortolotti L., Le persistenze della campagna romana: alcuni aspetti della sua evoluzione storica, intervento al seminario «Strumenti e metodi per la conoscenza della campagna romana», Dottorato di ricerca in Progettazione Paesistica, Roma 2000.

- Cafiero G., Conte G., L'agro romano nel PRG di Roma, in «Urbanistica Informazioni» n.152, mar.-apr., INU edizioni, Roma 1997, pp. 33-34.

- Cederna A., Brandelli d'Italia, s.e., Roma 1991.

- Ciccone F., Roma: capitale senza piano, in G. Campos Venutı, F. OlIVA (a cura di) "Cinquant'anni di urbanistica in Italia. 1942 1992», Laterza, Roma - Bari 1993, pp. 241-258. 
- Colarossi P., Belardelli F., lombardo N., abusivismo e ambiente, in A. ClementI, F. PEREGo (a cura di) «La metropoli "spontanea". Il caso di Roma", Dedalo, Roma 1983, pp. 104-119.

- Colasante D., 1925 - 1981: la città legale, in A. Clementi, F. PEREGo (a cura di) "La metropoli "spontanea". II caso di Roma», Dedalo, Roma 1983, pp. 249-279.

- Cuccia G., Urbanistica Edilizia Infrastrutture di Roma Capitale 1870 - 1990, Laterza, Roma - Bari 1991.

- Garano S., Leone A.M., Sebasti R., Visentini P. (a cura di), Roma: cronache urbanistiche degli anni settanta, Edizioni delle Autonomie, Roma 1979.

- INSOleRa I., Roma moderna, Einaudi, Torino 1971.

- INSOleRA I., Roma. Immagini e realtà dal $X$ al XX secolo, Laterza, Roma - Bari 1980.

- LeONe A., L'edificazione abusiva, in «Casabella» n.438, lug.-ago., Electa, Milano 1978, pp. 44-47.

- LEONE A.M. (a cura di), II recupero degli insediamenti abusivi, USPR - Documenti 1 (pubblicazione a cura del Comune di Roma), Roma 1981.

- LEONE A.M., I/ recupero urbanistico dei nuclei edilizi abusivi, in A.M. LEONE (a cura di), «ll recupero degli insediamenti abusivi», USPR Documenti 1 (pubblicazione a cura del Comune di Roma), Roma 1981, pp. 18-45.

- $\quad$ Lugl P.M., Urbanistica di Roma: trenta planimetrie per trenta secoli di storia, Bardi, Roma 1998.

- MANCINI A., INSOlERA I., Introduzione a trent'anni di storia urbanistica romana, in «Urbanistica» n.62, apr., INU edizioni, Roma 1974, pp. 64-106.

- Maderni M., Agro romano: la memoria storica dilapidata, in A. ClementI, F. PeREGo (a cura di), «La metropoli "spontanea". II caso di Roma», Dedalo, Roma 1983, pp. 100-103.

- Olivieri M., 1925 - 1981: la città abusiva, in A. Clementi, F. PEREGO (a cura di) «La metropoli "spontanea". II caso di Roma», Dedalo, Roma 1983, pp. 280-304.

- Olivieri M., Appia antica: cronologia di un parco mancato, in P. BERDINI (a cura di), «La città senza piano: le trasformazioni urbanistiche di Roma negli anni '80", Istituto Nazionale di Urbanistica - sezione Lazio, Roma 1992, pp. 87-112

- QUARONI L., Immagine di Roma, Laterza, Roma - Bari 1969. 
- SANFILIPPo M., Le tre città di Roma. Lo sviluppo urbano dalle origini ad oggi, Laterza, Roma-Bari 1993.

- VISENTINI P., La formazione dell'abusivismo nel territorio romano, in A.M. LEONE (a cura di), «ll recupero degli insediamenti abusivi», USPR - Documenti 1 (pubblicazione a cura del Comune di Roma), Roma 1981, pp. 6-17.

\section{SULLE “CAMPAGNE” DELL'AREA ROMANA OGgI}

- $\quad$ AA.VV., Roma per te. Itinerari storici e ambientali circoscrizione per circoscrizione, pubblicazione a cura del Comune di Roma, Roma 1996.

- CAlzolaRI V., Le interrelazioni e la varietà, in V. CALZOLARI «Storia e natura come sistema: un progetto per il territorio libero dell'area romana», Àrgos, Roma 1999, pp. 101-134.

- Cotti A., Roma anni novanta: produzione, servizi, mobilità, aree verdi e la nuova forma della città, Università La Sapienza, Roma 1999.

- Di Giovine M. (a cura di), Guida al verde di Roma, Lozzi \& Rossi, Roma 2000.

- Ferranti C., Paolella A. (a cura di), la pianificazione del Bacino del fiume Tevere 1992-2000, Gangemi, Roma 2000.

- Marocco M., Politiche e strumenti di intervento nelle aree agricole peri-urbane, in «Agricoltura Ambiente» n.8, giu., F.lli Palombi, Roma 1980, pp. 30-56.

- Olivieri M. (a cura di), Piano per il parco dell'Appia antica, Italia Nostra - sezione di Roma, Roma 1984.

- Piano Regolatore Generale: Guida alla progettazione negli ambiti di paesaggio - G7, (a cura del Comune di Roma - Ass.to Politiche per il territorio), Roma 2001.

- Piano Territoriale Paesistico: ambito territoriale n.2 - Litorale nord, (a cura della Regione Lazio - Ass.to Urbanistica e casa), Roma 1986.

- Piano Territoriale Paesistico: ambito territoriale n.4 - Valle del Tevere, (a cura della Regione Lazio - Ass.to Urbanistica e casa), Roma 1986. 
- Piano Territoriale Paesistico: ambito territoriale n.7 - Monterotondo Tivoli, (a cura della Regione Lazio - Ass.to Urbanistica e casa), Roma 1986.

- Piano Territoriale Paesistico: ambito territoriale n.15 - Roma, (a cura della Regione Lazio - Ass.to Urbanistica e casa), Roma 1987.

- TAgliaferRi C., I casali della campagna romana, Pieraldo, Roma 1991.

- Thiery A. (a cura di), Roma Salaria: la città delle ville, F.lli Palombi, Roma 2001.

- Ventriglia U., Idrogeologia della provincia di Roma, Regione Vulcanica dei Colli Albani, (a cura della Provincia di Roma - Ass.to LL.PP. Viabilità e Trasporti), Roma 1990.

\section{Sulla "campagna delle tenute"}

- Antonimi M., La riserva naturale Decima-Malafede: la selvaggia bellezza di un angolo dell'agro romano, s.l., 1998.

- BARASSe A., I casali di Tor Vergata: per una storia del suo territorio, in «Roma. Ricerca e formazione» n.12, dic., Università degli Studi Roma Tre, Roma 1999, pp. 23-25.

- Bertelli P.O., La Tenuta del Cavaliere: una storia della campagna romana, Monsignori, Roma 1995.

- CAfiero G. (a cura di), Agricoltura e natura intorno alla città di Crustumerium. La riserva naturale della Marcigliana, in «Urbanistica Quaderni» n.37, mar., INU edizioni, Roma 2003, pp. 177-194.

- Cafiero G. (a cura di), Ambienti fluviali e comunità urbane. La Riserva naturale della Valle dell'Aniene, in «Urbanistica Quaderni» n.37, mar., INU edizioni, Roma 2003, pp. 195-207.

- Cafiero G. (a cura di), Decima - Malafede. Il parco delle acque: natura e agricoltura nella cintura urbana, in «Urbanistica Quaderni» n.37, mar., INU edizioni, Roma 2003, pp. 154-166.

- CAfiero G. (a cura di), Paesaggio, natura e agricoltura di fronte alla pressione urbana. Tenuta dei Massimi, in «Urbanistica Quaderni» n.37, mar., INU edizioni, Roma 2003, pp. 129-142.

- Carbone F., FrassinetTI M., I parchi naturali di Roma. Atlante fotografico delle quattordici aree naturali protette di RomaNatura, a cura dell'Ente, Roma 2001. 
- D'Alessandro A., I proprietari delle tenute dell'Agro Romano nel 1783, in «Rivista di storia dell'agricoltura» n.4, a.IX, 1969, pp. 363380.

- FILENI E., Tipo tradizionale di gestione di una tenuta dell'Agro Romano, in «Rivista di storia dell'agricoltura» n.4, a.III, 1963, pp. 68-74.

- Gross H., Roma nel Settecento, Laterza, Roma - Bari 1990.

- Maderni M., Evoluzione storico-ambientale del territorio nordorientale della campagna romana, in P.O. BERTELLI, «La Tenuta del Cavaliere: una storia della campagna romana», Monsignori, Roma 1995, pp. 129-140.

- Paratore E., II suburbio geo-agrario di Roma, Istituto di Studi Romani, Roma 1979.

- PARIS T. (a cura di), L'area tiburtina: media valle dell'Aniene, monti Cornicolani, Sabina Romana, Officina edizioni, Roma 1978.

- Passigli S., Ricostruzione cartografica e paesaggio del Catasto Alessandrino, in «Archivio della Società Romana di Storia Patria» vol. 114, 1991, pp. 161-184.

- Rızo G.G., Qualità paesaggistico - ambientali e frange urbane nella periferia ovest di Roma, estratto da "Metodi e verifiche di bilancio ambientale urbano», s.l., 1991, pp. 93-120.

- Roccasecca P., Urbe, Suburbio e Agro: confini in movimento, dal sito digilander.libero.it/aperture/articoli/2.17.html.

- $\quad$ Scotoni L., Le tenute della Campagna Romana nel 1600. Saggi di ricostruzione cartografica, in «Società Tiburtina di Storia e Arte» vol. 59, 1986, pp. 185-262.

- Thiery A. (a cura di), Roma Salaria: la città delle ville, F.lli Palombi, Roma 2001.

- Vallino F.O., Melella P., Tenute e paesaggio agrario nel suburbio romano sud-orientale dal sec.XIV agli albori del Novecento, in «Bollettino della Società Geografica Italiana» vol.XII, Roma 1983.

\section{Sulla "campagna della bonifica"}

- $\quad$ Bagnasco C. (a cura di), Il delta del Tevere: un viaggio fra passato e futuro, F.lli Palombi, Roma 1998.

- Bellotti P., Il delta del Tevere: geologia, morfologia, evoluzione, in C. BAGNASCO (a cura di), «ll delta del Tevere: un viaggio fra passato e futuro», F.lli Palombi, Roma 1998, pp. 19-29. 
- Gisotti G., Geomorfologia e suoli, in ItAlia Nostra - SeziOne ROMANA, "Capocotta ultima spiaggia: proposta per il parco naturalistico-archeologico del litorale romano», Quasar, Roma 1985, pp. 39-41.

- Gisotti G., Collamarini D., Suolo e vegetazione nella Tenuta di Castelporziano, Edagricole, Bologna 1982.

- Gisotti G., Vegetazione, in Italia Nostra - Sezione Romana, «Capocotta ultima spiaggia: proposta per il parco naturalisticoarcheologico del litorale romano», Quasar, Roma 1985, pp. 42-48.

- italia nostra - Sezione Romana, Capocotta ultima spiaggia: proposta per il parco naturalistico-archeologico del litorale romano, Quasar, Roma 1985.

- Menegoni P, Pietrelli L., Alcuni aspetti naturalistici del delta tiberino, in C. BAGNASCO (a cura di), «ll delta del Tevere: un viaggio fra passato e futuro», F.lli Palombi, Roma 1998, pp. 32-54.

- Parisi Presicce A., Villetti G., Le bonifiche: un ponte fra passato e futuro, in C. BAGNASCO (a cura di), «ll delta del Tevere: un viaggio fra passato e futuro», F.lli Palombi, Roma 1998, pp. 97-109.

- Valenti G., I/ sistema agrario dell'Agro Romano, in P. BeVILAcQua e M. Rossı DorIA (a cura di), «Le bonifiche in Italia dal '700 a oggi», Laterza, Roma - Bari 1984, pp. 212-233.

\section{Sulla "campagna della città metropolitana"}

- Bertollini M., Parisi Presicce A., Da periferia a città, in C. BAGNASCO (a cura di), «ll delta del Tevere: un viaggio fra passato e futuro», F.lli Palombi, Roma 1998, pp. 121-143.

- Cafiero G. (a cura di), La Riserva naturale del Laurentino-Acqua Acetosa: i "ponti" del Laurentino 38, le origini di Roma, il paesaggio della campagna romana, in «Urbanistica Quaderni» n.37, mar., INU edizioni, Roma 2003, pp. 167-176.

- Capannelli E., Lo spreco delle aree non urbanizzate e le attività agricole, in AA.VV. «Roma, il piano delle periferie: materiali per un dibattito», L'ed edizioni, Roma 1993, pp. 47-53.

- Colasante D., 1925 - 1981: la città legale, in A. Clementi, F. PEREGo (a cura di) "La metropoli "spontanea". II caso di Roma», Dedalo, Roma 1983, pp. 249-279.

- Comune di Roma, Atlante delle periferie, (pubblicazione a cura dell'Ente), Roma 2003. 
- Cuccia G., Urbanistica Edilizia Infrastrutture di Roma Capitale 1870 - 1990, Laterza, Roma - Bari 1991.

- LeONe A., L'edificazione abusiva, in «Casabella» n.438, lug.-ago., Electa, Milano 1978, pp. 44-47.

- LeONE A.M. (a cura di), II recupero degli insediamenti abusivi, USPR - Documenti 1 (pubblicazione a cura del Comune di Roma), Roma 1981.

- MAdeRNi M., Evoluzione storico-ambientale del territorio nordorientale della campagna romana, in P.O. BERTELLI, «La Tenuta del Cavaliere: una storia della campagna romana», Monsignori, Roma 1995, pp. 129-140.

- Nucci C., Galassi A., Il Parco regionale dei Castelli Romani, in B. CIGNINI, G. MASSARI, S. PIGNATTI (a cura di) «L'ecosistema Roma: ambiente e territorio", F.lli Palombi, Roma 1995, pp. 281-292.

- Nucci C., Settore nord-orientale dell'area romana, in «Quaderni di Urbanistica Informazioni» n.8, INU edizioni, Roma 1990, pp. 216220.

- Olivieri M., 1925 - 1981: la città abusiva, in A. Clementi, F. PEREGO (a cura di) «La metropoli "spontanea". II caso di Roma», Dedalo, Roma 1983, pp. 280-304.

- Paris T. (a cura di), L'area dei Castelli Romani: gli insediamenti storici dei Colli Albani, Officina edizioni, Roma 1981.

- Rızzo G. G., La cicuta non c'è più: le trasformazioni/distruzione del vulcano lazlale, in «Bollettino del DUPT della Facoltà di Architettura di Firenze» n.2, Dipartimento di Urbanistica e Pianificazione del Territorio, Firenze 1996, pp. 30-36.

- $\quad$ Rizzo G.G., Qualità paesaggistico - ambientali e frange urbane nella periferia ovest di Roma, estratto da «Metodi e verifiche di bilancio ambientale urbano», 1991, pp. 93-120.

- VISENTINI P., Caratteri insediativi delle espansioni abusive, in «Casabella» n.438, lug.-ago., Electa, Milano 1978, pp. 48-53. 


$$
\ldots
$$




\section{ESITI E POSSIBILI INDIRIZZI DI SVILUPPO}

Questa ricerca non pretende ovviamente di dare risposte definitive sul tema del paesaggio in generale e del paesaggio agrario in particolare, ma cerca di offrire una possibile chiave di lettura a proposito di un tema che è oggi al centro di un ricco dibattito interdisciplinare.

$\mathrm{Si}$ è ritenuto utile condurre questo lavoro ad una serie di sintetiche argomentazioni conclusive, con le quali i presupposti metodologici iniziali, i passaggi principali e le applicazioni condotte possano esser messi fra loro in relazione, all'interno di un quadro unitario.

L'ipotesi di partenza ha assunto le risorse e i caratteri propri del paesaggio agrario - inteso come sistema di relazioni fra componenti fisiche, morfologiche, colturali, storiche - come elementi di ordine e qualità ambientale, funzionale e formale nella tutela e nell'organizzazione del territorio della campagna romana, sia nelle sue parti ancora libere, sia nelle sue parti già antropizzate.

Lo studio si è, dunque, articolato in due fasi principali, nelle quali le argomentazioni e le ipotesi iniziali sono state poi applicate ad un particolare ambito di studio.

La prima fase si è incentrata sugli aspetti teorici e metodologici: si è infatti partiti dalla definizione del paesaggio agrario e dalla descrizione dei suoi elementi componenti, per poi soffermarsi sulle modifiche della sua struttura e dei suoi 
processi formativi, fino ad arrivare ai tempi più recenti, quando è stato riconosciuto al paesaggio agrario un importante ruolo strategico nel processo di riqualificazione ambientale e paesistica intrapresa dal nostro paese.

Nella seconda fase del lavoro si è cercato di dare applicazione pratica a quanto espresso nella parte metodologica: è stata condotta una serie di descrizioni ed osservazioni inerenti le relazioni - fisico-morfologiche e storiche - che danno origine alle diverse tipologie di paesaggi agrari secondo le quali è possibile articolare oggi la campagna romana, riconoscendo a questa porzione di territorio una forte connotazione paesistica e attribuendole il ruolo di importante risorsa sia a motivo della sua estensione, sia per i suoi caratteri peculiari.

La ricerca ha, dunque, voluto mettere in relazione un possibile modello conoscitivo riferito al paesaggio agrario con un quadro territoriale specifico e ricco di situazioni paesistiche emblematiche, ponendosi l'obiettivo generale di valutare la fattibilità tanto dell'applicazione delle chiavi di lettura proposte per ciò che riguarda il paesaggio agrario, quanto della reale forza e ruolo di quest'ultimo all'interno di un processo di riqualificazione ambientale e paesistica.

Ai fini dell'applicabilità di questi elementi metodologici occorre mettere in evidenza alcuni aspetti che sono risultati determinanti nell'affrontare le problematiche indagate nell'ambito di studio. 
L'articolazione dei "nuovi ruoli" individuati per il paesaggio agrario fa sì che esso venga considerato una risorsa per la protezione dell'ambiente e del territorio, un elemento sostanziale per l'equilibrio economico generale, un sistema strutturante per la riconoscibilità e l'identità storica dei luoghi. Piuttosto che limitarsi ad una visione che non tenga conto della mutabilità del paesaggio agrario - e della domanda di trasformazione alla quale un ambito come quello romano è sottoposto, a contatto come è con l'influsso di una grande città - si dimostra opportuno far riferimento ad una serie di scenari differenziati, calibrati sugli elementi componenti la loro struttura e sulle potenzialità insite in ciascuno di essi.

Roma per un lungo periodo ha avuto un rapporto particolare con la sua campagna, attribuendole un ruolo esclusivamente produttivo: pascolo innanzitutto, coltivazioni estensive in secondo luogo. Oggi, invece, il rapporto è profondamente mutato. Nello specifico della realtà romana, presa come ambito applicativo emblematico di una realtà agricola ben più ampia, le "campagne romane» individuate e descritte si pongono in maniera differente:

- vi è il caso di porzioni consistenti di campagne che sono delimitate da importanti elementi morfologici o insediativi e sono solo marginalmente toccate da episodi urbani interni, nelle quali il paesaggio agrario rappresenta ancora oggi la regola strutturale;

- vi sono ambiti di territori agricoli circoscritti quasi completamente da situazioni urbane, nei quali, però, l'attività agricola e gli elementi paesistici risultano ancora particolarmente consistenti; 
- vi sono residui di campagne interstiziali, contenuti nelle forme invasive ed irrisolte dell'urbanizzato e presenti soprattutto nei settori periferici della città, in prossimità delle grandi infrastrutture moderne.

All'interno di un processo di riqualificazione ambientale e paesistica, in una realtà così articolata e complessa, il paesaggio agrario diventa il tessuto fondamentale per riconoscere alcune situazioni-tipo e per definire gli approcci congruenti per ciascuna di esse.

Una prima situazione è rappresentata dai "paesaggi agricoli della diversità biologica e colturale"1, dove agricoltura sostenibile, ambiente e paesaggio sono sistematicamente intrecciati e dove sostanzialmente l'agricoltura partecipa ancora attivamente alla stabilità ed alla struttura complessa dei sistemi ecologici e paesistici complessivi e strutturanti.

$\mathrm{Nel}$ caso della campagna romana ricalcano questa situazione-tipo i casi descritti per la «campagna delle tenute", che, come abbiamo visto, struttura ancora molti ambiti territoriali dell'area romana, e per la «campagna della bonifica».

Sono questi, dunque, i paesaggi per i quali diventa necessario e vitale affermare il valore della diversità agraria come elemento indissolubile nel quale rintracciare le regole e le matrici storiche: le forme tradizionali degli impianti, la cura del suolo e delle sue sistemazioni, la riconoscibilità dei

\footnotetext{
Le definizioni sono in parte desunte da quanto affermato da L. CARAVAGGI nel volume curato dal Ministero per i Beni e le Attività Culturali, Conferenza Nazionale per il Paesaggio - Lavori preparatori, Gangemi, Roma 2001, pp.85-92.
} 
rapporti tra insediamenti, collegamenti, spazi coltivati e spazi boschivi, l'immagine di efficienza delle infrastrutture a servizio della produzione agricola: ovvero tutte quelle componenti che costituiscono, tra l'altro, preziosi esempi di razionalità tecnica ed ambientale.

Questi paesaggi sono, in parte, paesaggi in progressiva riduzione. Non è infatti inusuale, soprattutto in alcune tenute, la presenza di una serie di attività agricole produttive che tendono ad uscire dalla contemporaneità, a diventare antieconomiche. In queste realtà, la scarsa redditività delle colture in rapporto all'alto costo di manutenzione del suolo coltivato induce fenomeni di abbandono secondo una tendenza che potrebbe apparire inarrestabile.

È dunque essenziale, in questi contesti, attivare processi attraverso i quali arrestare il fenomeno regressivo e procedere ad una sorta di ri-conversione, che passi attraverso un'attribuzione di nuove funzioni, di nuovi ruoli sociali ed economici, al fine di recuperare importanti e significativi brani di un paesaggio agrario che è già "dismesso" o è evidentemente in via di dismissione.

Il loro mantenimento deve essere dunque connesso ad un sostanziale riconoscimento del loro effettivo ruolo, che non può più essere esclusivamente economico-produttivo, ma che deve essere ecologico, storico, paesistico. In questo senso si rivela essenziale una lettura dell'intero "sistema paesaggio agrario" che proceda per elementi componenti, per relazioni, per legami strutturanti. 
Una seconda situazione è quella dei "paesaggi agrari da recuperare" ${ }^{2}$, ovvero quelle aree agricole che possono acquistare evidenti significati di presidio paesistico nei contesti sottoposti ad una forte pressione insediativa.

In questi contesti, dei quali l'area romana è ricchissima e che sono stati qui descritti nel caso della «campagna della città metropolitana», le attività connesse all'agricoltura perdono stabilità e redditività per effetto dell'attesa delle trasformazioni future e l'immagine ricorrente è quella dall'abbandono o dalla marginalità.

Questo tipo di paesaggio agrario ha, però, in sé diverse potenzialità, fra le quali spicca il ruolo di elemento ordinatore dell'urbanizzazione diffusa. Insinuandosi fra gli insediamenti, infatti, i "residui agricoli" diventano un ostacolo al dilagare delle espansioni - abusive o regolari che siano - e contribuiscono a dare una forma compiuta al tessuto urbano.

Gli stessi elementi componenti il paesaggio agrario - canali, recinzioni naturali, viali alberati, campi coltivati - costruiscono una sorta di reticolo connettivo all'interno del quale si possono inserire gli spazi verdi pubblici fra le zone edificate, e realizzano quei "corridoi ecologici" che sono necessari per mantenere o ricostruire la continuità fra le diverse parti degli ecosistemi naturali, oggi separate proprio dall'urbanizzazione diffusa.

Considerare il paesaggio agrario come il paradigma di riferimento, adattabile alle diverse situazioni territoriali entro

2 L. CaravagGi, op.cit. 
le quali è necessario attivare un processo di riqualificazione ambientale e paesistica, necessita di una definizione più appropriata di alcune questioni rimaste ancora aperte, che potrebbero essere indagate nell'ambito di altre esperienze di studio, teorico ed applicato.

Una questione da ritenersi prioritaria riguarda ad esempio la realizzabilità degli approcci indagati al momento in cui l'agricoltura non è più una risorsa produttiva, ma il paesaggio agrario diventa "solo" un brano da conservare a memoria del passato oppure un elemento di una connettività ambientale più estesa.

Mantenere "stabili" alcune porzioni dei paesaggi più popolati ed antropizzati, ad esempio, richiede una faticosa e costosa azione di cura e manutenzione, che il più delle volte è del tutto ingiustificata dal punto di vista dei risultati economici, e può risultare conflittuale con le stesse aspettative sociali e culturali più diffuse.

Appare inoltre evidente che solo

pochi territori possono essere sottratti dalla dinamica in divenire della continua modificazione del paesaggio, a meno di una improbabile ed impensabile imbalsamazione del territorio ${ }^{3}$.

È dunque necessario incentivare riflessioni e approcci che tengano conto di come possa essere rilevante e pertinente per molti territori (soprattutto per quelli più densamente urbanizzati e congestionati) la costruzione di una nuova e integrata immagine paesistica, nella quale le tracce del

3 A. LANZANI, Sette strategie per il paesaggio, in Alberto CLEMENTI (a cura di), «Interpretazioni di paesaggio», Meltemi, Roma 2002, p.266. 
passato agiranno come limite, come termine di confronto per l'azione trasformativa che, comunque, dovrà ri-plasmare il territorio, modificarlo e tenere conto del complesso intreccio di componenti offerti dall'attuale nuovo ordinamento socioterritoriale.

È essenziale, quindi, identificare queste nuove connotazioni paesistiche e attribuire loro il ruolo di portatrici di nuove identità territoriali all'interno degli inevitabili processi di trasformazione cui sono sottoposti il paesaggio ed il territorio tutto. 


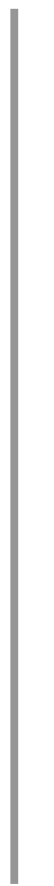

RIFERIMENTI ICONOGRAFICI

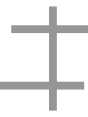




\section{PARTE PRIMA}

Figure 1, 2. Disegni tratti da G. FERRARA, L'architettura del paesaggio italiano, Marsilio, Padova 1968.

Figure 3, 7-9. P. FABBRI, Natura e cultura del paesaggio agrario, CittàStudi, Milano 1997.

Figure 4-6. Stralci della Carta Tecnica Regionale del Lazio, edita dalla Regione Lazio nel 1991.

Figura 10. http://europa.eu.int/comm/environment/newprg

\section{PARTE SECONDA}

Figura 1. Parziale rielaborazione di $A$. CAzzoLA dell'elaborato $\mathrm{G}_{9-3}$ del Nuovo Piano Regolatore Generale del Comune di Roma adottato in Consiglio Comunale il 20.3.2003.

Figure 2, 68. V. CALZOLARI, Storia e natura come sistema: un progetto per il territorio libero dell'area romana, Àrgos, Roma 1999.

Figure 3, 23, 29, 30, 35, 41, 47, 53, 63, 64, 66, 69, 72, 76. Elaborazioni di A. CAZZOLA.

Figure 4, 5. R. ALMAGiÀ, Lazio, UTET, Torino 1966.

Figure 6, 8. L. BORTOLOTTI, Roma fuori le mura, Laterza, Roma - Bari 1988.

Figura 7. Parziale rielaborazione di A. CAZzOLA tratta da V. GHISELLINI (a cura di), L'Autostrada del Grande Raccordo Anulare, USPR - Documenti 10 (pubblicazione a cura del Comune di Roma), Roma s.d.

Figure 9, 10, 12, 15. I. INSOLERA, Roma moderna. Un secolo di storia urbanistica, Einaudi, Torino 1971.

Figure 11, 14, 22. P.M. LUGLI, Urbanistica di Roma: trenta planimetrie per trenta secoli di storia, Bardi, Roma 1998.

Figure 13, 16, 17. Rielaborazioni di A. CAZZOLA tratte da A.M. LEONE (a cura di), II recupero degli insediamenti abusivi, USPR - Documenti 1 (pubblicazione a cura del Comune di Roma), Roma 1981.

Figure 18-21. F. CicCONE, Roma: capitale senza piano, in G. CAMPos VENUTI, F. OLIVA (a cura di) «Cinquant'anni di urbanistica in Italia. 1942 1992», Laterza, Roma - Bari 1993.

Figure 24, 31, 38, 40, 44, 45, 49, 54, 74. Foto di A. CAzzolA.

Figure 25, 27, 28, 33, 34, 39, 46, 50-52, 55, 56, 60-62, 71, 75. C. TAGLIAFERRI, I casali della campagna romana, Pieraldo, Roma 1991. 
Figure 26, 32. F. CARBONE, M. FrassinetTI, I parchi naturali di Roma. Atlante fotografico delle quattordici aree naturali protette di RomaNatura, a cura dell'Ente, Roma 2001.

Figure 36, 37, 42, 48. G. CAFIERO (a cura di), La pianificazione del sistema delle aree protette di Roma. Le nove Riserve naturali di RomaNatura, «Urbanistica Quaderni» n.37, mar., INU edizioni, Roma 2003.

Figura 43. AA.VV., Roma per te. Itinerari storici e ambientali circoscrizione per circoscrizione: vol.VIII, pubblicazione a cura del Comune di Roma, Roma 1996.

Figura 57. C. Bagnasco (a cura di), I/ delta del Tevere: un viaggio fra passato e futuro, F.lli Palombi, Roma 1998.

Figure 58, 59. Parziale rielaborazione di A. CAzzolA tratta da C. BAGNASCO (a cura di), Il delta del Tevere: un viaggio fra passato e futuro, F.lli Palombi, Roma 1998.

Figure 65, 67, 70, 73. Stralci della Carta Tecnica Regionale del Lazio, edita dalla Regione Lazio nel 1991. 